\title{
Le site de La Rochette à Mauron (Morbihan) : les multiples occupations d'un promontoire
}

The site of La Rochette at Mauron (Morbihan): Multiple Occupations of a Promontory

Jean-Yves Tinévez, Laurent Quesnel, Nancy Marcoux, Klet Donnart, Véronique Bardel, Maurice Gautier, Vincent Bernard, Michel Fontugne, Johannes Van der Plicht et Christine Oberlin

\section{(2) OpenEdition}

\section{Journals}

Édition électronique

URL : https://journals.openedition.org/rao/1581

DOI : $10.4000 /$ rao. 1581

ISBN : 978-2-7535-1846-9

ISSN : 1775-3732

Éditeur

Presses universitaires de Rennes

Édition imprimée

Date de publication : 31 décembre 2011

Pagination : 71-148

ISBN : 978-2-7535-1844-5

ISSN : 0767-709X

Référence électronique

Jean-Yves Tinévez, Laurent Quesnel, Nancy Marcoux, Klet Donnart, Véronique Bardel, Maurice Gautier, Vincent Bernard, Michel Fontugne, Johannes Van der Plicht et Christine Oberlin, « Le site de La Rochette à Mauron (Morbihan) : les multiples occupations d'un promontoire », Revue archéologique de I'Ouest [En ligne], 28 | 2011, mis en ligne le 30 mars 2014, consulté le 22 août 2022. URL : http:// journals.openedition.org/rao/1581; DOl : https://doi.org/10.4000/rao.1581 


\title{
Le site de La Rochette à Mauron (Morbihan) : les multiples occupations d'un promontoire
}

\author{
The site of La Rochette at Mauron (Morbihan): \\ Multiple Occupations of a Promontory
}

\author{
Jean-Yves Tinevez*, Laurent Quesnel ${ }^{* *}$, Nancy Marcoux**, Klet Donnart**, \\ Véronique Bardel ${ }^{* * *}$, Maurice Gautier ${ }^{* * * *}$, Vincent Bernard ${ }^{* *}$, \\ Michel Fontugne****, Johannes Van der PLichto***** \\ et Christine OberLin ${ }^{* * * * * * *}$
}

\begin{abstract}
Résumé : Découvert par prospection aérienne en 1992, le site de La Rochette révèle un ensemble de cinq fossés barrant un relief de promontoire dominant la vallée de l'Yvel. Le plus imposant de ces fossés est segmenté en quatre tronçons. Une fouille programmée de l'ensemble du promontoire est engagée sur la problématique de l'attribution chronoculturelle des éperons barrés et la nature de leurs occupations internes. L'étude fut menée de 2003 à 2007 sur une surface de 1,3 ha, soit $80 \%$ de l'emprise totale du site enclos. Le nombre important de structures de fondation mis au jour fait apparaitre une succession d'aménagements en arcs de cercles concentriques traduisant une parfaite adaptation à la topographie. Face à l'indigence du matériel archéologique datant, dix-sept datations par le radiocarbone viennent étayer l'attribution chronoculturelle des quatre principales occupations mises en évidence. Une première fréquentation, au Néolithique ancien, se résume à cinq foyers en cuvette groupés au nord-est du site. Au Bronze final, le large fossé interrompu constitue un imposant barrage très structuré abritant une série de petits bâtiments régulièrement disposés en arc de cercle. Le premier âge du Fer est marqué par l'implantation d'une palissade sur laquelle s'adossent de petits bâtiments et un large fossé ceinturant la pointe du promontoire au sud. Après un hiatus de plusieurs siècles, la partie sud est réinvestie par une imposante enceinte en bois protégeant une maison sur poteau. L'ensemble est bien daté (radiocarbone et dendrochronologie) du haut Moyen Âge, fin VII $^{\mathrm{e}}$-VIII ${ }^{\mathrm{e}}$ siècle apr. J.-C., et a subi une destruction systématique par incendie.

Une étude anthracologique détaillée permet d'intégrer chaque phase d'occupation dans son contexte environnemental.

Abstract: The site of la Rochette was discovered by aerial photography in 1992. Photographs revealed a group of five ditches defending a promontory settlement which dominates the Yvel valley. The most imposing ditch is divided into four segments. A research excavation carried out on the totallity of the headland was engaged with questions concerning the chronology of fortified promontories and the nature their internal occupation. Excavations were carried out between 2003 and 2007 on an area of $1,3 \mathrm{ha}$, about $80 \%$ of the entire enclosed site. The large number of structures discovered indicates a succession with several of them concentric, revealing a perfect adaptation to the local relief. Due to a lack of good dating material, seventeen radiocarbon dates contribute to the chronocultural determination of the four principal occupation phases revealed on the site. The first traces of early Neolithic occupation are limited to five shallow hearths grouped on the north-east of the site. During the late Bronze Age, the wide interrupted ditch forms a well structured

\footnotetext{
* Service régional de l'Archéologie de Bretagne, avenue Charles-Foulon, 35700 Rennes - UMR 6566 (CreAAH - Centre de Recherche en Archéologie, Archéosciences et Histroire, Université de Rennes 1, campus de Beaulieu) - CS 74205, 35042 Rennes Cedex.

** UMR 6566 (CreAAH - Centre de Recherche en Archéologie, Archéosciences et Histoire, Université de Rennes 1 - campus de Beaulieu, CS 74205 , 35042 Rennes Cedex.

*** Illustratrice -9 rue des Giroflées, 29800 Landerneau.

**** Archéologue aérien - Les Hauts de Breslon, 35470 Pléchâtel.

***** Laboratoire des Sciences du Climat (labo. mixte CEA-CNRS) - bâtiment 12, Avenue de la Terrasse, 91198 Gif-sur-Yvette.

****** Centrum voor isotopenonderzoek, Rijkuniversiteit, Groningen (Pays-Bas).

******* Centre de datation par le Radiocarbone, UMR 578 (Archéologie et Archéométrie), Université Claude Bernard-Lyon 1 - bâtiment 217, 69622 Villeurbanne.
} 
fortification, protecting behind its inner earthen rampart a series of small buildings placed in an arc. The early Iron Age is marked by the construction of a palisade backed by small wooden buildings and by the excavation of a wide ditch enclosing the southern end of the promontory. After a gap of several centuries, the southern part of the site is re-occupied by a huge wooden enclosure protecting a post-build house. The whole is precisely dated, by radiocarbon and dendrochronology, to the Early Mediaeval period, late VII ${ }^{\text {th }}$-early VIIt century AD. It was totally destroyed by fire. A detailed anthracological study placed each occupation phase in its environmental context.

Mots clé : éperon barré, Néolithique ancien, âge du Bronze, premier âge du Fer, haut Moyen Âge, Radiocarbone, Anthracologie, Dendrochronologie, fortifications, fossés, bâtiments, foyers, incendie.

Keywords : fortified promontory settlement, early Neolithic, Bronze Age, early Iron Age, early Mediaeval, anthracology, radiocarbon dating, dendrochronology, ramparts, palissades, wooden buildings, hearths, fire destruction.

\section{INTRODUCTION GÉNÉRALE}

Localisée en limite des départements du Morbihan, des Côtes-d'Armor et de l'Ille-et-Vilaine, la commune de Mauron se caractérise par un relief de plateau légèrement vallonné entre les collines des Landes du Méné au nordouest et les contreforts du massif forestier de Paimpont au sud-est. Région à forte composante agricole, le paysage s'est largement ouvert à la suite des remembrements intensifs réalisés dans les années soixante. L'uniformité relative de ce paysage est agrémentée par les nombreux méandres de la vallée de la rivière Yvel, issue du massif du Méné au nord-ouest et affluent de l'Oust au sud (fig. 1 et 2). Ce paysage privé de couvert bocager est particulièrement favorable à la prospection aérienne. Lors de l'une de ces missions au début des années 1990, Maurice Gautier a pour la première fois attiré notre attention sur le promontoire localisé à une centaine de mètres au sud du hameau de La Rochette (fig. 1). Par ses flancs escarpés surplombant un large méandre, l'extrémité du plateau est profondément marquée par la confluence de l'Yvel et l'un de ses petits affluents. Lors des périodes sèches, de nettes anomalies phytologiques y faisaient apparaitre le tracé de cinq fossés disposés en arcs de cercles parallèles et centrés sur la pointe du promontoire. Par son aspect segmenté rappelant les enceintes fossoyées à multiples interruptions, le fossé de barrage le plus externe et le plus large

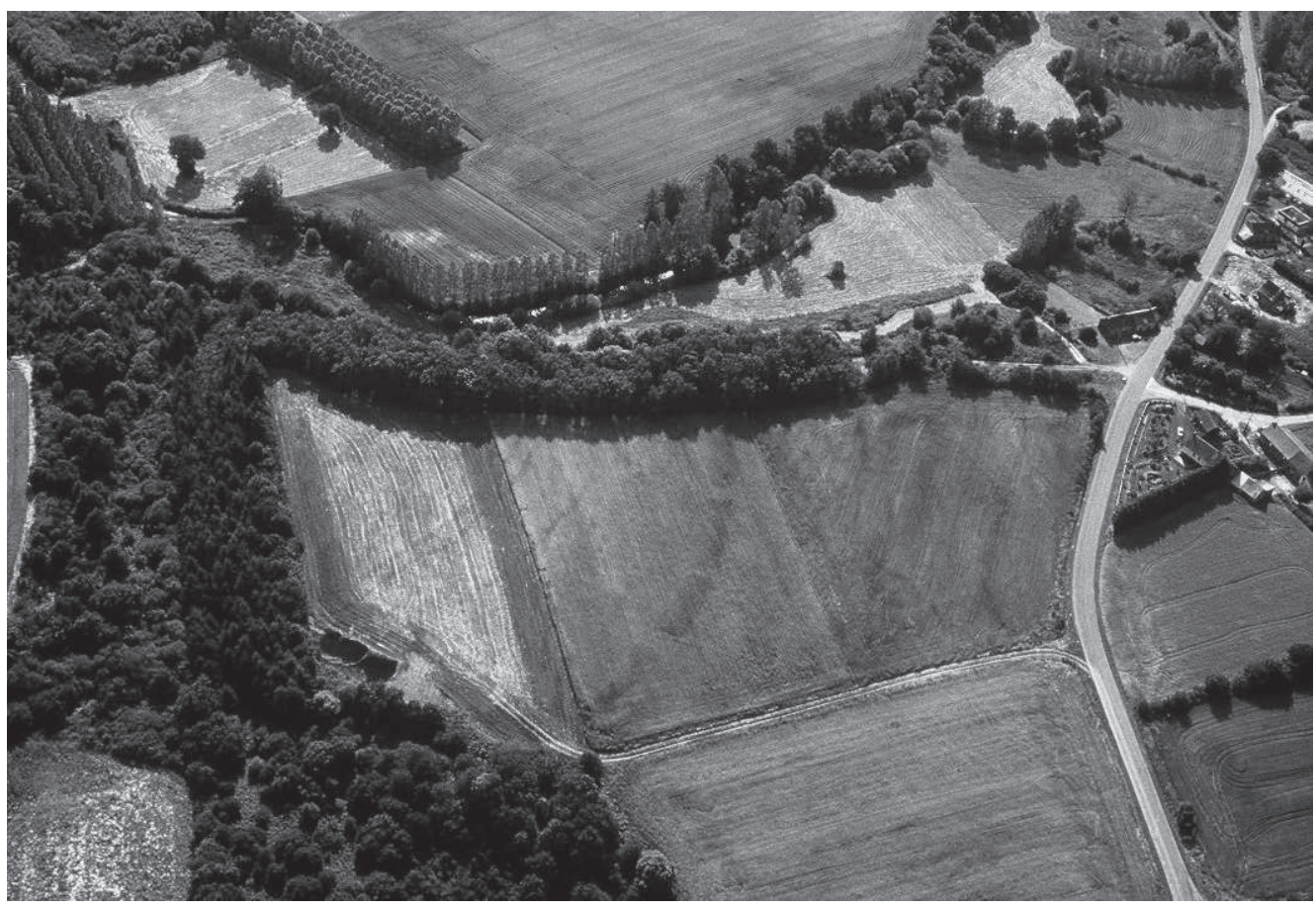

Figure 1 : Vue aérienne à partir du nord-est du promontoire de Mauron-La Rochette localisé à la confluence de l'Yvel (en arrière plan) et de l'un de ses petits affluents en bordure sud. Les cinq fossés de barrage sont visibles par anomalies phytologiques (cliché Maurice Gautier, 1992).

Figure 1: Aerial view from north-east of Mauron-La Rochette promontory located at the confluence of river Yvel (in background) with one of its little tributaries on the south edge. The five dam ditches can be seen by cropmarks. 

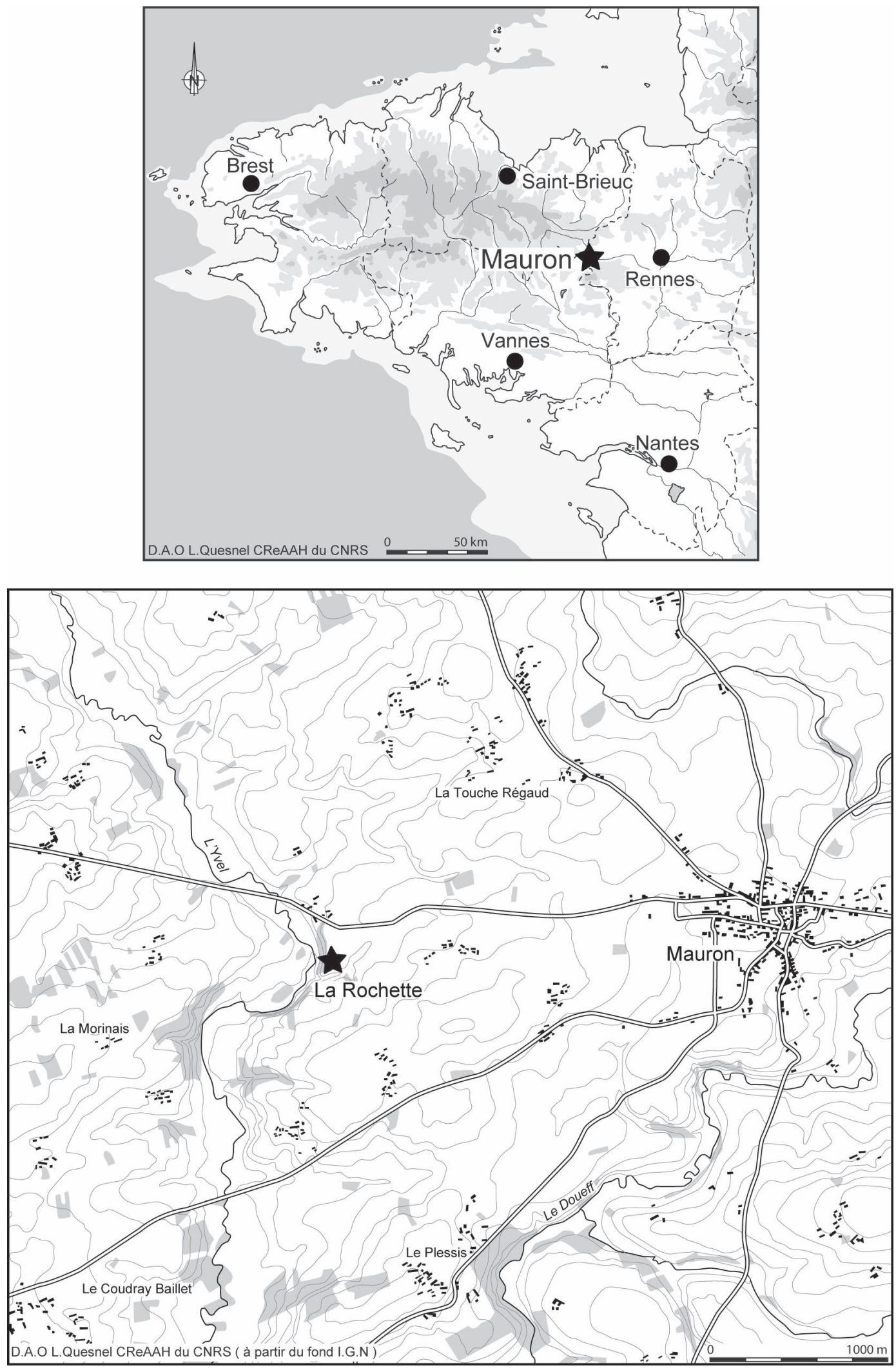

Figure 2 : Localisation du site de La Rochette à l'ouest de la commune de Mauron (Morbihan). Figure 2: La Rochette site localization in the west of Mauron commune (Morbihan). 
a plus précisément retenu notre attention dans le cadre des recherches sur les formes de l'habitat de la préhistoire récente et de la protohistoire ancienne. À la suite de sondages d'évaluation réalisés en 2003 , une opération programmée extensive a été menée de 2004 à 2007. Si les résultats diffèrent notablement des objectifs escomptés, cette opération met en évidence une succession d'occupations sur ce site naturel particulièrement convoité et révèle des vestiges d'aménagements pour la plupart inédits sur le plan régional.

Sur un ensemble de plus de cinq cents faits archéologiques découverts, cinq occupations successives du promontoire ont été mises en évidence, du Néolithique ancien au bas Moyen Âge. Le Néolithique ancien n'est en réalité représenté que par cinq structures de combustion regroupées dans la partie nord-est du site et isolées de tout contexte. Dans la partie nord, un habitat fortifié par le système de barrage segmenté est attribué au Bronze final et remarquablement structuré. Ces premiers aménagements de grande ampleur vont modeler le paysage et s'imposeront probablement dans la configuration des occupations suivantes. Les aménagements du premier âge du Fer, constitués essentiellement d'une palissade avec quelques constructions adossées et d'un large fossé en pointe d'éperon, restent énigmatiques en l'absence de réelles structures d'habitat internes.

Après un abandon de plusieurs siècles, la pointe du promontoire est à nouveau convoitée au haut Moyen Âge pour y implanter un habitat protégé d'une enceinte triangulaire à structure de bois massive. Après la destruction violente de cette fortification par le feu, le site sera plus légèrement investi au bas Moyen Âge par l'installation de petits enclos palissadés.

\section{Première partie \\ Problématique, historique de l'opération ET MÉTHOdOLOGIE (J.-Y. T.)}

Dès la découverte du site par prospection aérienne au début des années 1990, la morphologie du grand fossé externe, segmenté en quatre tronçons bien apparents, et sa position en barrage d'un éperon naturel faisaient référence à de nombreux sites similaires. Les enceintes fossoyées à entrées multiples se comptent par dizaines dans le centre-ouest de la France et elles sont attribuées généralement, mais pas exclusivement, au Néolithique récent et final. Plusieurs d'entreelles ont fait l'objet de sondages et de fouilles. En Bretagne, le développement de la prospection aérienne et les conditions favorables de certaines années sèches, comme 1989 par exemple, ont permis de reconnaître de rares exemples comparables. On notera en Ille-et-Vilaine les sites de La Trappe à
Boistrudan et de La Charonnière à Saint-Aubin-des-Landes (Leroux, 1992), délimitant grâce à deux fossés concentriques régulièrement interrompus des emprises respectives de 1,2 et 3 ha en position topographique dominante en bord de plateau. Les promontoires barrés sont également très nombreux, en zones côtières tout particulièrement et, lorsqu'ils font l'objet d'expertise, nombreux sont ceux qui révèlent une succession d'occupations. Jusqu'au début des années 2000, très rares étaient les exemples connus d'éperons barrés d'un fossé segmenté à l'image de celui de La Rochette et le plus proche était alors celui du Val Aubin sur la commune de Lamballe (Hamon, 2001). Depuis, l'inventaire s'est étoffé de plusieurs sites comparables à la fois sur les plans de la morphologie, de la position topographique et de la surface occupée.

Nos connaissances sur ce type de site restant indigentes dans la région, tant sur la nature et la fonction des structures que sur l'attribution typochronologique du matériel associé, une autorisation d'opération programmée a été sollicitée dans le cadre des recherches sur les formes de l'habitat de la Préhistoire récente et la Protohistoire ancienne menée au sein de l'UMR 6566.

\section{LES SONDAGES D’ÉVALUATION EN 2003}

L’opération programmée en 2003 avait pour objet la réalisation de larges sondages d'évaluation sur les trois principaux systèmes de barrages révélés par les clichés aériens et leur environnement immédiat. Quatre sondages, A à D, totalisant une surface de $620 \mathrm{~m}^{2}$ ont été ouverts et fouillés (fig. 3 et 4). L'objectif de l'opération étant principalement axé sur l'étude du fossé segmenté $2 \mathrm{~b}$, les deux sondages principaux ont été réalisés sur ses extrémités est (sondage $\mathrm{A}$ sur le tronçon 2b.4) et ouest (sondage B sur le tronçon 2b.1).

Le double fossé $4 \mathrm{~b}-4 \mathrm{c}$ et le fossé 5 ont été évalués par les deux sondages complémentaires, $\mathrm{C}$ et $\mathrm{D}$, sur leurs extrémités occidentales en bordure du promontoire. Le sondage $\mathrm{D}$, au sud, a d'emblée permis d'attribuer le fossé 5 à l'âge du Fer grâce à la présence de quelques fragments de céramique. En revanche, sur le fossé segmenté au nord, l'attribution chronologique est restée au début sujette à caution. La morphologie de la structure et sa dynamique de comblement ont été rapidement maîtrisées à partir des deux sondages et des coupes transversales correspondantes. La nature différente des excavations et de leurs comblements entre le nord et le sud du site permettait d'ores et déjà d'entrevoir au moins deux phases d'occupation. Mais l'indigence du mobilier, mêlant des éléments attribuables au Néolithique (roches taillées, blocs de dolérite, quelques fragments de céramique) et des pièces plus récentes, ne permettait pas d'être affirmatif 
Figure 3 : Topographie du site et emprise des différentes phases de l'opération de fouille programmée, des sondages d'évaluation en 2003 aux dernières interventions de terrain en 2007 sur les secteurs sud.

Figure 3: Site topography and location of the different excavation phases from the 2003 evaluation sondages to the last 2007 diggings in in the south of the site.

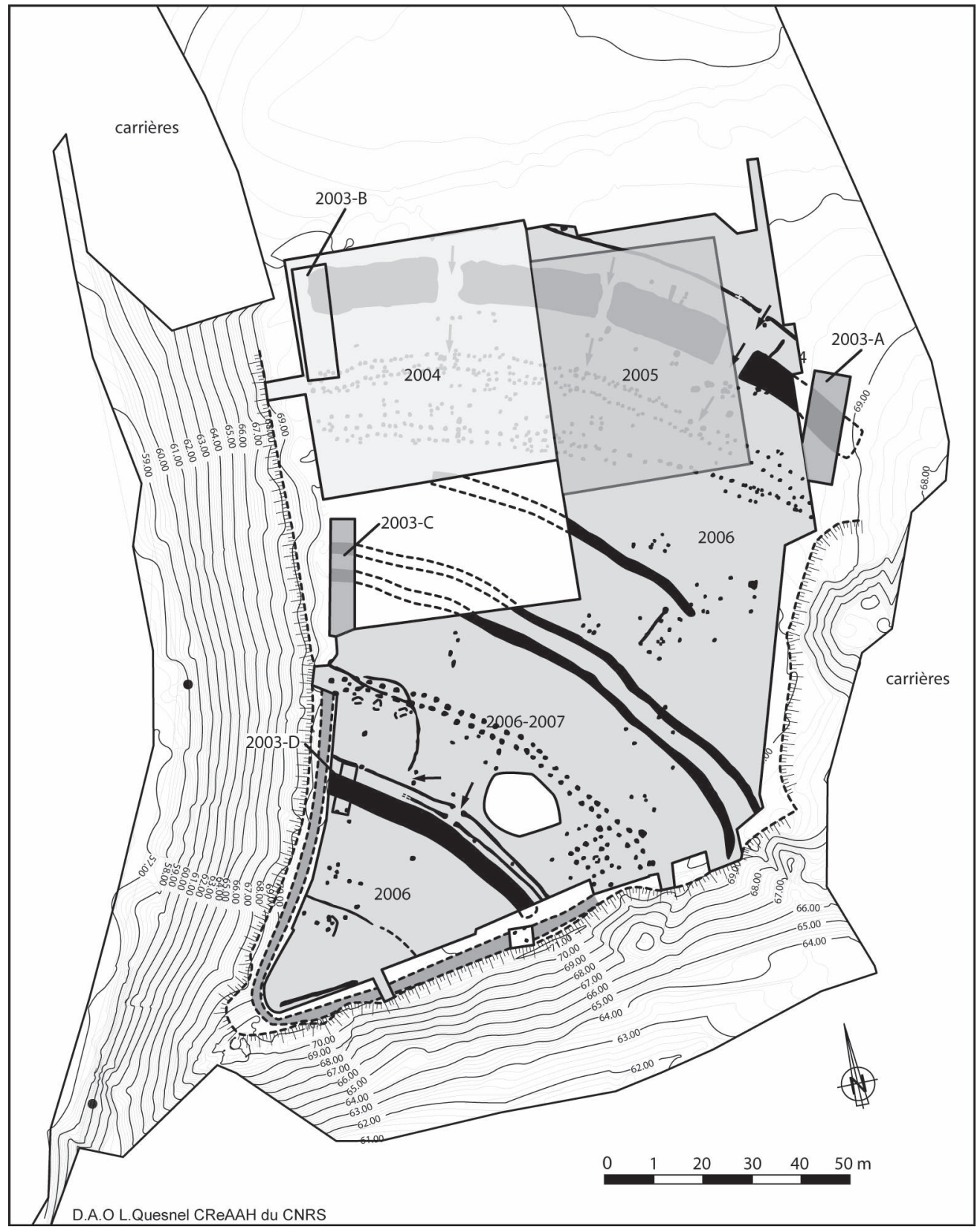

sur la datation de ce système de barrage inédit dans la région. Par ailleurs, quelques trous de poteau alignés, mis au jour en limite du sondage B au nord-ouest, laissaient présumer de structures bâties en zone interne, hypothèse largement confirmée dès la première campagne de fouille en 2004.

\section{LE PROGRAMME TRISANNUEL 2004-2006 ET LA MÉTHODOLOGIE ADOPTÉE}

$\mathrm{Au}$ vu des résultats positifs des sondages d'évaluation, l'objectif de l'opération triannuelle 2004-2006 était, dans un premier temps, la datation et l'étude morphologique des structures fossoyées, de leur dynamique de comblement et d'un éventuel phasage dans le cas d'une occupation multiple. Les dimensions importantes en largeur et profondeur des éléments du fossé interrompu argumentaient en faveur d'une stratigraphie conséquente bien préservée, susceptible de fournir des données novatrices sur la chronologie régionale des cultures de la Préhistoire récente. Dans un second temps, l'objectif était d'étendre les recherches en partie interne du site, à la recherche de vestiges d'occupation dont quelques éléments avaient été pressentis en 2003 en limite sud du sondage B au nord-ouest. 


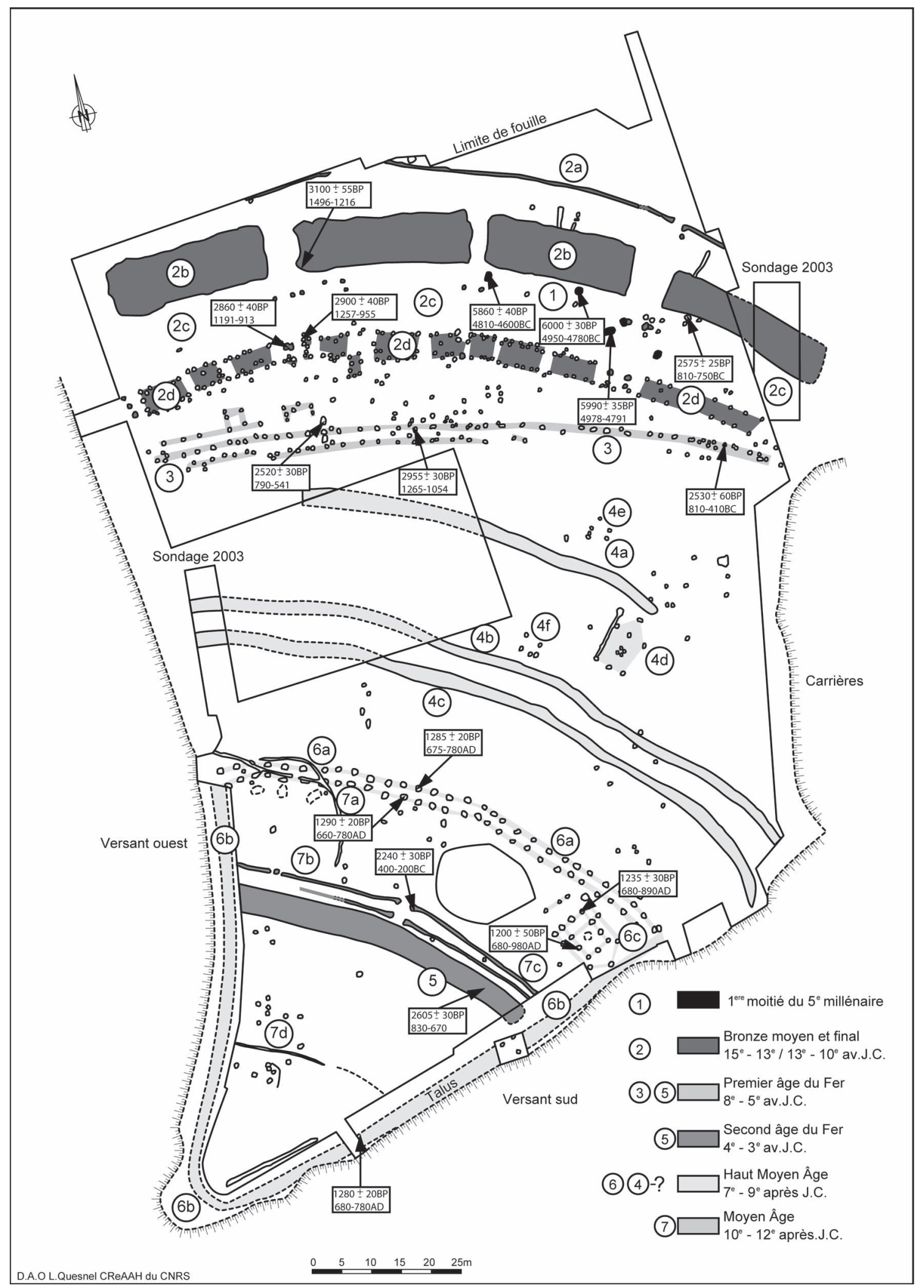

Figure 4 : Ensembles chrono-culturels mis au jour et localisation des datations par le radiocarbone. Figure 4: Chrono-cultural wholes discovered and location of Radiocarbon datings. 
Afin de mener à bien ces deux objectifs complémentaires, les deux premières campagnes de fouille, 2004 et 2005, ont été partagées entre une série de sondages manuels dans le fossé segmenté et une étude extensive de la partie nord du site (fig. 3). La campagne 2006, avec quelques interventions complémentaires en 2007, a été consacrée principalement à la partie centrale et sud jusqu'à la pointe du promontoire.

Lors du démarrage de l'opération, la totalité de l'emprise du site, de statut juridique privé, était soumise à une activité agricole intense. Ainsi, à l'exception d'un petit talus préservé en bordure sud et ouest du promontoire, aucun mouvement de terrain d'apparence anthropique n'était visible. Les flancs du promontoire sont excavés par endroits par des carrières de schistes abandonnées et une large carrière, en activité il y a peu, a entamé le rebord oriental du site sur une profondeur de $4 \mathrm{~m}$ environ. Par ailleurs, plusieurs anciens agriculteurs nous ont signalé la présence de forts talus ceinturant jadis l'extrémité du plateau. Secteur longtemps abandonné en friches, il fut remis en culture à la suite du remembrement des années 1960, responsable de l'arasement des talus et du comblement des fossés. Ces témoignages corroborent le cadastre napoléonien sur lequel deux limites parcellaires en arc de cercle isolent la pointe, en distorsion complète avec l'orientation d'un parcellaire régulier sur le plateau (fig. 5). Face à ce constat, la technique de décapage extensif habituellement utilisée sur les sites étendus en milieu rural fut mise en œuvre, précédée toutefois d'une prospection des terres labourées et d'un relevé topographique (fig. 3).

\section{A. Un relevé topographique préalable}

En 2003, en préalable à l'intervention de terrain, une opération de relevé topographique a été réalisée par l'IUT Génie Civil de Rennes dans le cadre du diplôme de fin d'études universitaires (fig. 3). D'une durée de quatre jours, cette opération a couvert la partie haute du promontoire et ses abrupts sud et sud-ouest. En raison de la végétation estivale trop dense, le relevé des pentes nord-ouest et est n'avait pu être achevé; une intervention complémentaire a été réalisée en mars 2004. Parallèlement à ces relevés, la zone de fouille 2004 a été positionnée précisément. Malgré ce complément, les deux secteurs fortement entaillés par d'anciennes carrières au sud et au nord-ouest n'ont pu être topographiés en raison d'une broussaille trop dense.

Les courbes de niveau espacées de 0,20 m mettent en évidence les légers vallonnements du plateau. D'une altitude moyenne de $70 \mathrm{~m}$ à l'emplacement du fossé interrompu, le relief s'élève à 70,80 $\mathrm{m}$ vers la pointe de l'éperon. Sur les pentes, le dénivelé varie de $9 \mathrm{~m}$ au sud-est à une quinzaine de mètres à la pointe de l'éperon et sur le flanc ouest, lequel présente une pente d'environ $40 \%$ en surplomb de la rivière. La forte déclivité des versants ouest et sud de ce promontoire en fait un retranchement défensif privilégié surplombant la vallée de l'Yvel.

\section{B. Les décapages extensifs}

En 2003, le secteur B, ouvert en bordure nord-ouest du site, avait l'avantage d'associer un segment du grand fossé et une première série de trous de poteau à environ $12 \mathrm{~m}$ vers l'intérieur de l'enceinte.

En 2004, ce secteur a ainsi été privilégié pour l'extension des recherches (fig. 3). Une emprise carrée de $2500 \mathrm{~m}^{2}(50 \mathrm{x}$ $50 \mathrm{~m}$ ) a été ouverte en mars 2004 en bordure de l'abrupt nord-ouest du site : décapage mécanique au godet lisse, nettoyage manuel, repérage, relevé en plan et fouille par moitié des anomalies mises au jour. Un carroyage fixe (carrés de $5 \mathrm{~m}$ de côté) y sera étendu au fur et à mesure de l'avancement des travaux.

En 2005, la zone d'étude fut étendue vers l'est sur une emprise supplémentaire de $2000 \mathrm{~m}^{2}(50 \times 40 \mathrm{~m})$. La surface ainsi obtenue couvre la totalité des trois tronçons occidentaux du grand fossé et l'essentiel des structures internes associées.

En 2006, la surface étudiée a été portée à $8000 \mathrm{~m}^{2}$ environ. L'étude de cette surface importante a pu être menée grâce à deux facteurs : l'étude du grand fossé nord nécessitant un investissement important s'est achevée en 2005 et de vastes secteurs en partie centrale du site se sont révélés quasiment vierges de vestiges.

Par ailleurs, la fouille 2006 a été complétée d'interventions ponctuelles en 2007 avant la remise en état des terrains. Cette dernière campagne a permis d'étudier la partie centrale, l'extrémité sud jusqu'aux abrupts de l'éperon, mais également de compléter les données sur la zone nord-est. Le stockage des terres a nécessité la réserve d'une banquette d'environ $1500 \mathrm{~m}^{2}$ au centre et une seconde plus réduite au sud du site.

Sur ce décapage extensif d'une surface de 1,3 ha, soit environ $80 \%$ de l'emprise totale du site enclos, réalisé entre 2003 et 2006, les structures en creux ont été systématiquement relevées en plan et la fouille des structures a été menée à un degré jugé suffisant pour la compréhension des ensembles mis au jour.

\section{LOCALISATION, GÉOLOGIE ET ENVIRONNEMENT NATUREL (J.-Y. T.)}

\section{A. Topographie et orographie}

Le site de La Rochette occupe un promontoire naturel de forme triangulaire limité par la confluence de l'Yvel et d'un petit 


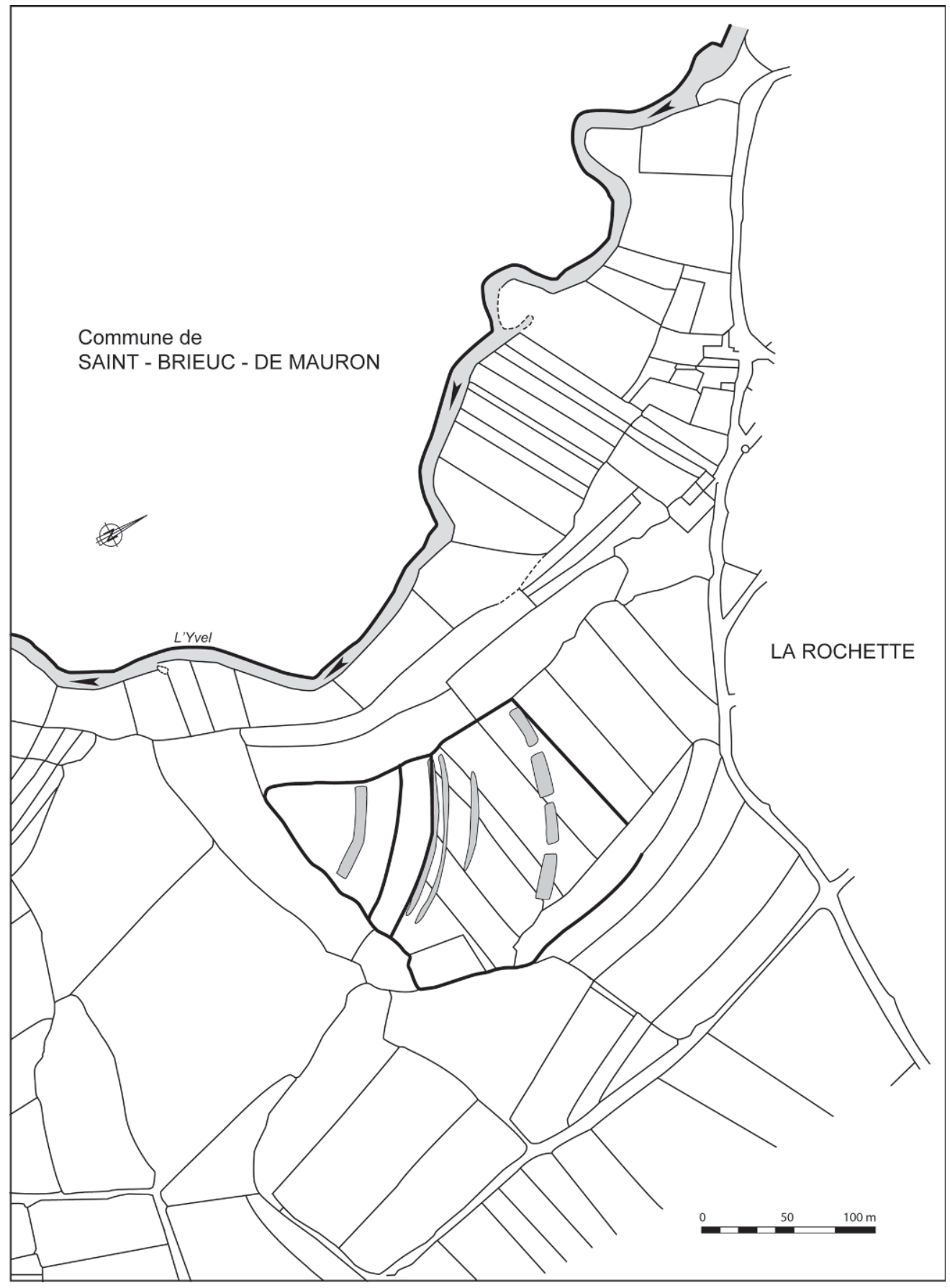

Figure 5 : Report des anomalies phytologiques sur le cadastre du XIX ${ }^{e}$ siècle : deux parcelles en arc de cercle soulignent le tracé des vestiges en pointe de promontoire; en revanche, le fossé segmenté ne conditionne nullement l'orientation du parcellaire. Figure 5: Cropmarks report on the $19^{\text {th }}$ century cadastre plan: two arched pieces underline the traces on promontory point but the interrupted ditch is independant from field system.

ruisseau, en limite de la commune de Saint-Brieuc-de-Mauron. Le promontoire est ainsi bordé d'escarpements boisés au sud et surtout à l'ouest, dominant de neuf à quinze mètres la vallée de l'Yvel, petit cours d'eau prenant sa source dans les collines du Méné au nord et affluent de l'Oust au sud (fig. 1, 2, 5, 6).

La carte IGN n ${ }^{\circ} 1018 \mathrm{O}$ (Merdrignac) positionne le site aux coordonnées Lambert $\mathrm{x}=253,3 ; \mathrm{y}=1052,9-\mathrm{z}=70 \mathrm{~m}$ NGF. Le site occupe la quasi-totalité des parcelles $n^{\circ} 50$ et 55 de la section XB du cadastre de 1971 (fig. 6). Sur le cadastre napoléonien (fig. 5), une parcelle en arceau se superpose quasi parfaitement au tracé du couple de fossés parallèles $4 \mathrm{~b}-4 \mathrm{c}$, indiquant probablement l'existence d'un barrage encore en élévation au XIx ${ }^{e}$ siècle et corroborant le témoignage des anciens agriculteurs. En revanche, au nord du site, le fossé segmenté $2 b$ et son rempart ne laissent aucune trace dans l'orientation de ce parcellaire, ce qui indique certainement un impact plus atténué dans le paysage aux époques récentes. 
Figure 6 : Report des anomalies phytologiques sur le cadastre actuel (parcelles XB 50 et XB 55, couvrant l'emprise du promontoire dominant la vallée de l'Yvel). Figure 6: Cropmarks report on the actual cadastre plan (pieces $X B 50$ and XB 55 covering the promontory above the Yvel valley).

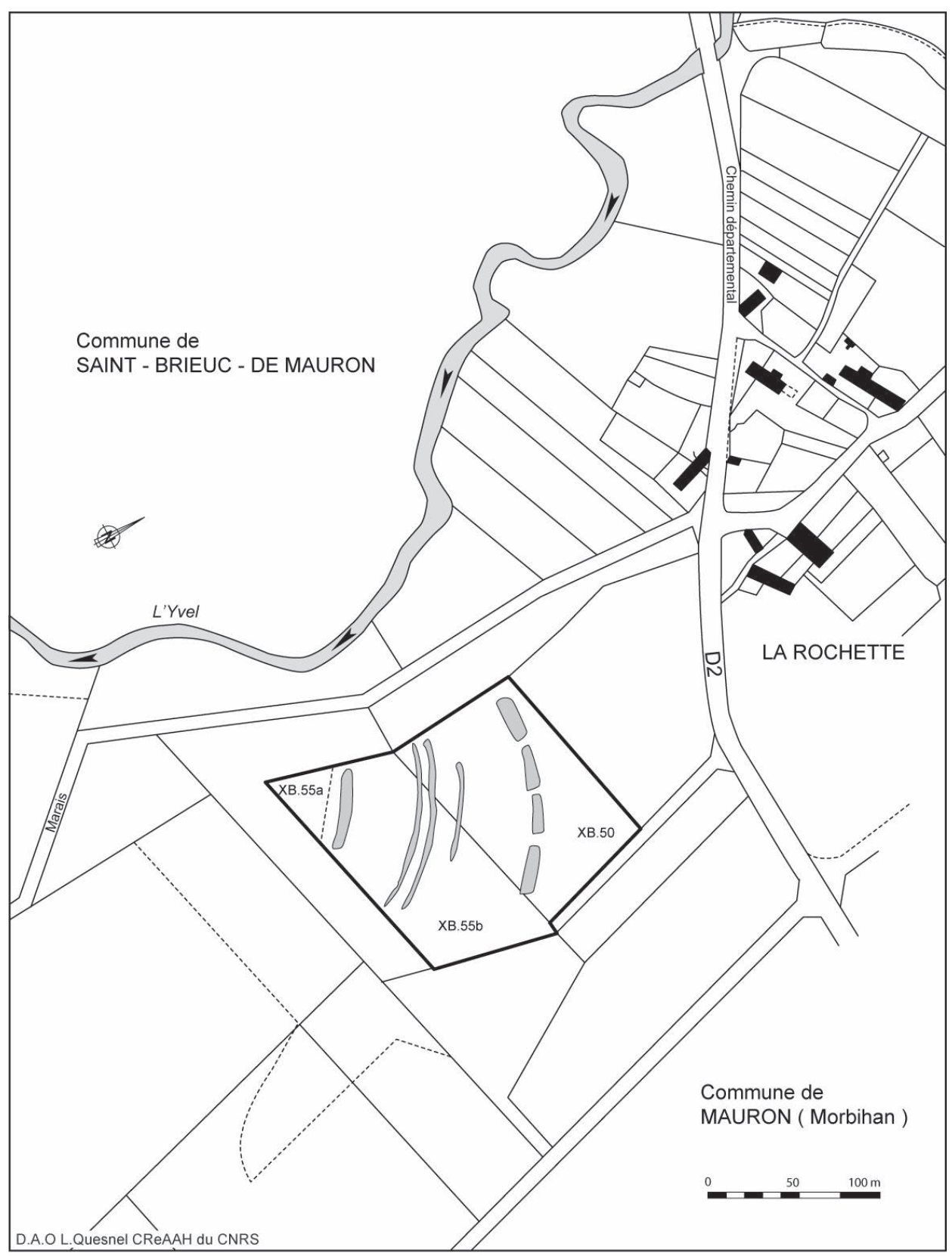

\section{B. La géologie du site}

La région de Mauron est localisée au coeur du domaine structural centre-armoricain, ici constitué de terrains sédimentaires protérozoïques et paléozoïques. Le site est implanté sur la formation dite du Briovérien de Bretagne centrale, que l'on retrouve depuis le Finistère sud (le Porzay) et le Morbihan occidental (secteur de Gourin) jusqu'en Maine-et-Loire, au nord d'Angers (Carte géologique de la France à $1 / 50000$, feuille Saint-Méen-le-Grand, $n^{\circ} 315$; Thomas et Carn, 2008). Du point de vue lithologique, il s'agit d'une alternance de bancs gréseux et silto-argileux. Le secteur de La Rochette appartient au faciès connu régionalement sous le nom de "Dalles de Néant " et constitué de siltites et argilites de couleur vert sombre.

Les massifs granitiques les plus proches sont localisés à une quinzaine de kilomètres au nord-ouest pour le massif de Plémet-Goméné (granite dit de Ménéac, à biotite et muscovite), en limite des départements du Morbihan et des Côtesd'Armor, d'une part et, d'autre part, à plus d'une vingtaine de kilomètres au sud-ouest pour le granite de Lizio-La Villeder, en rive droite de l'Oust. Le massif de Goméné, sur 
le versant sud des collines du Méné, est également le lieu où l'Yvel prend sa source et constitue ainsi indirectement une origine d'approvisionnement en matériau granitique par l'intermédiaire de blocs charriés et scellés dans les alluvions de l'Yvel. L'une des meules du site est ainsi façonnée sur un gros galet de granite (voir infra, $\$$ VII E).

Sur le promontoire, le substrat se présente sous la forme d'un schiste gris-vert, peu résistant et se délitant en plaquettes orientées est-ouest. L'érosion de zones plus tendres est à l'origine d'une légère ondulation de la surface du plateau, légers reliefs qui ont pu être mis à profit lors des diverses occupations. Au nord du site archéologique, l'une de ces légères dépressions naturelles est traversée en écharpe du nord-ouest au sud-est par une veine de sédiment jaune clair très induré et contenant de petits galets de quartz roulés tandis que la partie centrale du promontoire est marquée d'un filon de quartz blanc orienté est-ouest. Dans le grand fossé segmenté, la présence de nombreux galets de quartz éclatés sous l'action de la chaleur indique probablement un apport anthropique à partir des basses terrasses de l'Yvel.

\section{LE CONTEXTE ARCHÉOLOGIQUE (M.G., J.-Y. T.)}

L'inventaire archéologique du vaste territoire de Mauron et des communes environnantes - Gaël (Ille-et-Vilaine), Concoret et Saint-Léry (Morbihan) à l'est; Illifaut (Côtesd'Armor) au nord; Brignac, Saint-Brieuc-de-Mauron, Guilliers et Néant-sur-Yvel (Morbihan) à l'ouest et au sud - montre des disparités importantes dans la répartition et la nature des sites (fig. 7).

Les périodes anciennes sont peu représentées. Le seul dolmen recensé à Mauron, près du village de La Saudraie au sud-est de la commune et non loin de la lisière de la forêt de Paimpont, a été détruit lors du remembrement et sa morphologie reste indéterminée. Un peu plus à l'est, sur la commune de Concoret, les restes dégradés de « l'allée couverte" du Rocher ne permettent pas de bien définir son architecture (Gouézin, 1994). Cependant, la mise au jour d'outils lithiques néolithiques lors des travaux agricoles est fréquente dans le secteur avec notamment une hache-marteau en roche tenace au lieu-dit Les Landelles, à quelques centaines de mètres au nord de La Rochette.

À partir des années 1970, les profondes mutations agraires mises en place à la suite des remembrements offraient des perspectives toutes nouvelles à la détection aérienne à basse altitude et l'inventaire archéologique de Mauron s'est alors nettement étoffé à partir des années 1990. Ainsi, plus de $90 \%$ des soixante dix-sept entités archéologiques actuellement recensées sur son territoire ont été découvertes lors des missions de prospections aériennes menées par Maurice Gautier (2002). Les anomalies phythologiques ont révélé de nombreuses structures fossoyées dont le plan est parfois spectaculaire. Leur répartition couvre préférentiellement la partie ouest de la commune, le long de la vallée de l'Yvel. Plus de $50 \%$ des sites sont matérialisés par des enclos dont la morphologie est à dominante quadrangulaire et certains d'entre eux sont associés à un gisement de matériel antique. Les enclos au tracé curvilinéaire représentent $30 \%$ des entités et semblent commander un réseau parcellaire périphérique. Cette densité de sites repérés en aérien s'étend vers l'ouest sur les communes de Saint-Brieuc-de-Mauron et Guilliers où, sur les vingt neuf et soixante sept entités respectivement recensées, les enclos fossoyés en représentent plus de $90 \%$. La répartition sur les plateaux parait ainsi homogène de part et d'autre de la vallée de l'Yvel.

On notera la complexité de certains ensembles fossoyés comme la double enceinte ovale de La Tesserais, celle du Coudray-Baillet ou le site de La Bande du Verger constitué de cinq enclos emboîtés, sur la commune de Mauron par exemple, ou la triple enceinte semi-circulaire de La Jannaie des Bois au nord de Saint-Brieuc-de-Mauron. Bien que leur datation reste indéterminée pour la plupart en l'absence de sondages, la densité des sites et leur ampleur sont révélatrices d'une très forte occupation protohistorique et antique. Les découvertes anciennes comme celle du trésor de monnaies coriosolites de Mauron, localisé au Petit-Valet (Galliou et al., 2009) et l'étude, plus récente, des deux bustes gaulois de Saint-Utel (Ménez, 1999) confortent l'impression d'une fréquentation très dense de la vallée de l'Yvel à la fin de l'âge du Fer. Une concentration particulière de ces entités majeures couvre l'extrémité sud-ouest du territoire de Mauron, de part et d'autre de la vallée de l'Yvel en limite des communes de Saint Brieuc-de-Mauron à l'ouest et de Néant-sur-Yvel au sud. Ainsi, le promontoire de la Rochette est il au cœur d'un contexte archéologique riche et diversifié. En revanche, la partie nord-est de la commune est quasiment vide d'indices, mais cela peut découler d'une lisibilité plus difficile en raison de la présence de nombreuses zones boisées et d'un paysage encore largement bocager.

Non loin du site de La Rochette, plusieurs tronçons de voies matérialisées par les fossés de bordure ont été vus sur des missions IGN de 1976 (Gautier, 1993). L'un de ces tronçons, qui borde à l'est le promontoire, est directement connecté à la voie antique Rennes-Quimper dont le tracé suit une orientation nord-est/sud-ouest, en bordure de la crête topographique. Un tronçon de même orientation a été repéré au lieu-dit La Métairie des Landes à l'ouest de SaintBrieuc-de-Mauron, puis en limite occidentale de Guilliers au lieu-dit Les Liotais. La voie antique et le réseau viaire secondaire semblent ici servir d'ossature à un vaste aména- 


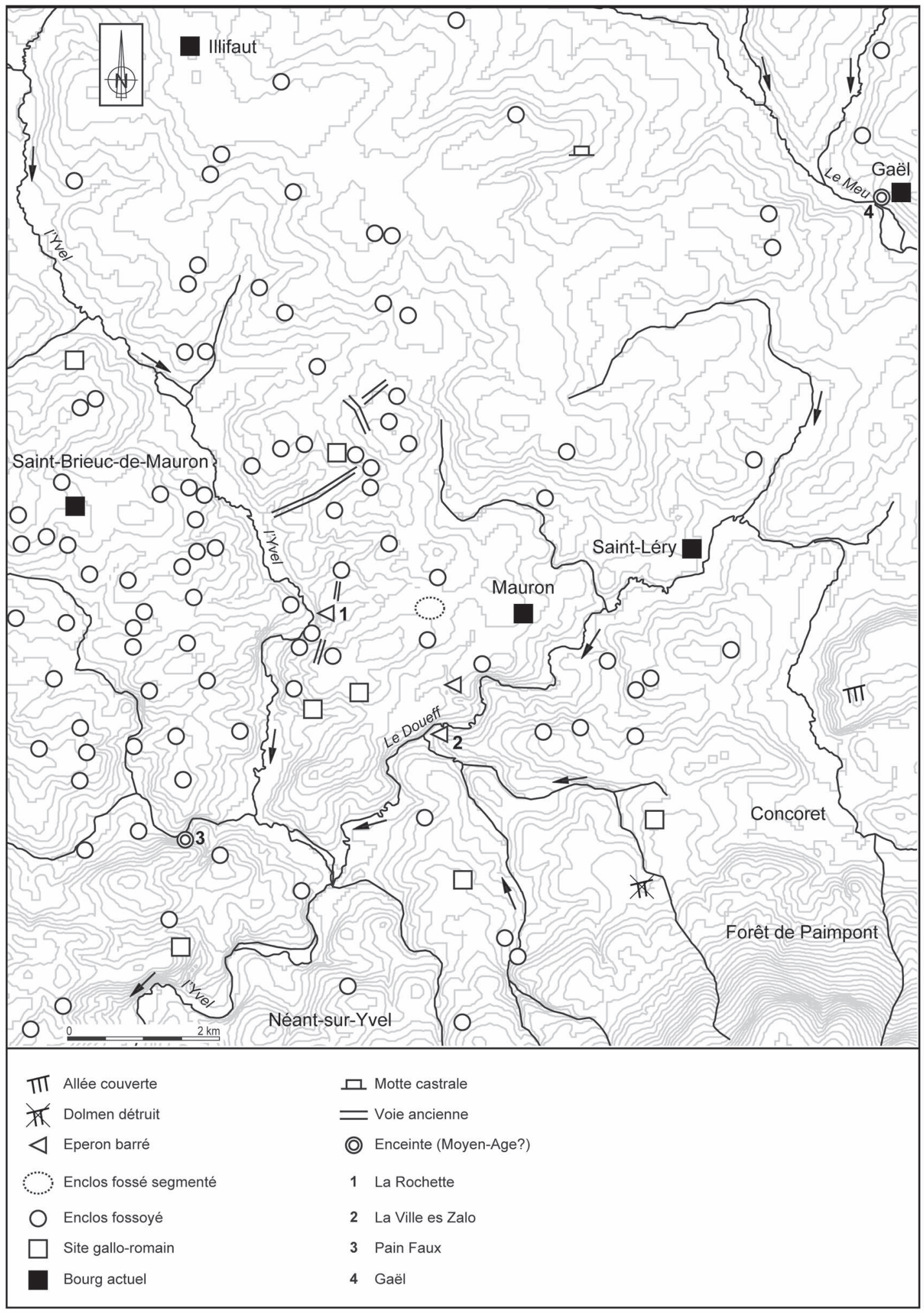

Figure 7 : Contexte du site de La Rochette, d'après l'inventaire archéologique de la commune de Mauron et des communes voisines, en limite des départements du Morbihan, d'Ille-et-Vilaine et des Côtes-d'Armor, reporté sur fond oro-hydrographique.

Figure 7: Context of La Rochette site from the archaeological inventory of Mauron and nearby communes, reported on oro-hydrographic map. 
gement agraire de près de 800 hectares, hélas non daté par l'archéologie (Gautier, 1996). Cependant, le paysage semble fortement structuré à l'époque romaine et la présence d'un tel réseau de voies, avec carrefour et passage à gué sur l'Yvel, à proximité du site, n'est probablement pas sans incidence sur la nature de ses occupations ultérieures, au haut Moyen Âge notamment.

Localisées respectivement à un kilomètre à l'est et à deux kilomètres au sud-est de La Rochette, les deux enceintes fossoyées du Bignon et de La Ville-ès-Zalo présentent un plan ovalaire à plusieurs interruptions. La morphologie du second site est particulièrement proche de celle de La Rochette : son fossé interrompu barre l'accès à un promontoire dominant la vallée du Doueff et une large zone marécageuse (cf. infra, fig. $27, \mathrm{n}^{\circ} 2$ ). Un site de même nature est localisé à $6 \mathrm{~km}$ au sud-ouest, sur la commune de Guilliers, lieu-dit Quenanque, en bordure du ruisseau de Rézo.

Deux enceintes peuvent être attribuées à la période médiévale. Celle de Painfaux, au sud-ouest de Mauron, est une fortification ovalaire de $35 \times 20 \mathrm{~m}$ et, au sud du bourg de Gaël, sur la rive gauche du Meu, une enceinte quadrangulaire dénommée " Château de Judicaël » entourait le champ de foire mais est aujourd'hui détruite (Banéat, 1928, p. 79-80).

\section{Deuxième partie LES RÉSULTATS ARCHÉOLOGIQUES (J.-Y.T.)}

\section{Caractères générauX DES STRUCTURES ET DU MOBILIER, ATTRIBUTIONS CHRONOLOGIQUES}

En raison de la nature du sous-sol et du contact direct entre la terre labourée et le toit du substrat schisteux sans couche de transition, les vestiges ne sont décelables qu'au niveau de ce dernier. Sa fragilité et ses variantes d'altération ont rendu le décapage délicat pour bien distinguer certaines fondations dont le comblement ne contraste que faiblement par rapport à l'encaissant. C'est le cas notamment des trous de poteau de l'âge du Bronze au nord du site, mais également des fondations de la double palissade sud malgré leurs dimensions importantes et leur datation, nettement plus récente, du haut Moyen Âge. Du fait de cette nature ingrate du substrat, certaines anomalies enregistrées se sont révélées des leurres à la fouille (irrégularités du schiste, impact de racines, altérations...); ceci explique certaines discontinuités dans la numérotation des faits archéologiques.
La morphologie des trous de poteau peut varier en fonction de l'état de l'encaissant, y compris pour un même ensemble cohérent. Ainsi, la bande de schiste altérée et tendre, au nord, révèle des fondations circulaires et régulières; dans les secteurs se délitant en plaquettes, les contours des creusements sont plus anguleux et irréguliers, quelle que soit la période concernée. D’une façon générale, les trous de poteau sont à parois verticales ou fortement inclinées et à fond plat plus ou moins régularisé. Quelques-uns présentent des traces d'écrasement en fond de creusement, à l'image du site néolithique de La Hersonnais à Pléchâtel où l'utilisation d'outils en pierre est attestée sur un substrat de nature proche; mais ces observations restent nettement minoritaires et les traces d'arrachement de substrat sont bien plus fréquentes sans que l'on puisse déceler la trace d'un outil particulier.

Un nombre important de structures en creux a ainsi été mis au jour : les cinq fossés visibles sur les clichés aériens, plus de deux cents mètres de tranchée de fondation étroite (palissades), treize structures de combustion en cuvette et plus de cinq cents trous de poteau. Le plan d'ensemble (fig. 8) fait apparaître des aménagements multiples en arcs de cercles concentriques dont la régularité et le parallélisme traduit à la fois une volonté de protection renforcée du promontoire, une adaptation parfaite aux caractéristiques naturelles du site et, pour certains ensembles, une contemporanéité des structures entre-elles.

Malgré la surface et le nombre de structures fouillées, le matériel archéologique est peu abondant et peu déterminant. La céramique, majoritaire, totalise 413 tessons ou ensembles provenant d'un même vase, pour un poids de 7030 g. Près de $80 \%$ proviennent de la zone nord, essentiellement de l'ensemble 2 attribué à l'âge du Bronze. Ce matériel est globalement très morcelé et érodé, et rares sont les éléments de formes ou de décors. Le mobilier lithique est limité à un total de 27 pièces taillées, 2 fragments de lames de hache polie et une quarantaine de fragments de macrooutillage en roches exogènes.

En raison de l'extrême indigence en mobilier datant, notamment pour la partie sud du site, et des rares cas de recoupements de vestiges permettant de distinguer une chronologie relative, l'interprétation et l'attribution chronologique des diverses phases d'occupation sont en grande partie tributaires des analyses, à partir du charbon de bois notamment (datations par le radiocarbone, anthracologie, dendrochronologie). Un total de dix-sept datations par le radiocarbone a ainsi été obtenu.

À partir de ces résultats, plusieurs ensembles cohérents témoignent d'occupations successives étroitement liées à la topographie des lieux. Afin d'en faciliter la lecture, nous prenons le parti de décrire les ensembles reconnus en fonction 


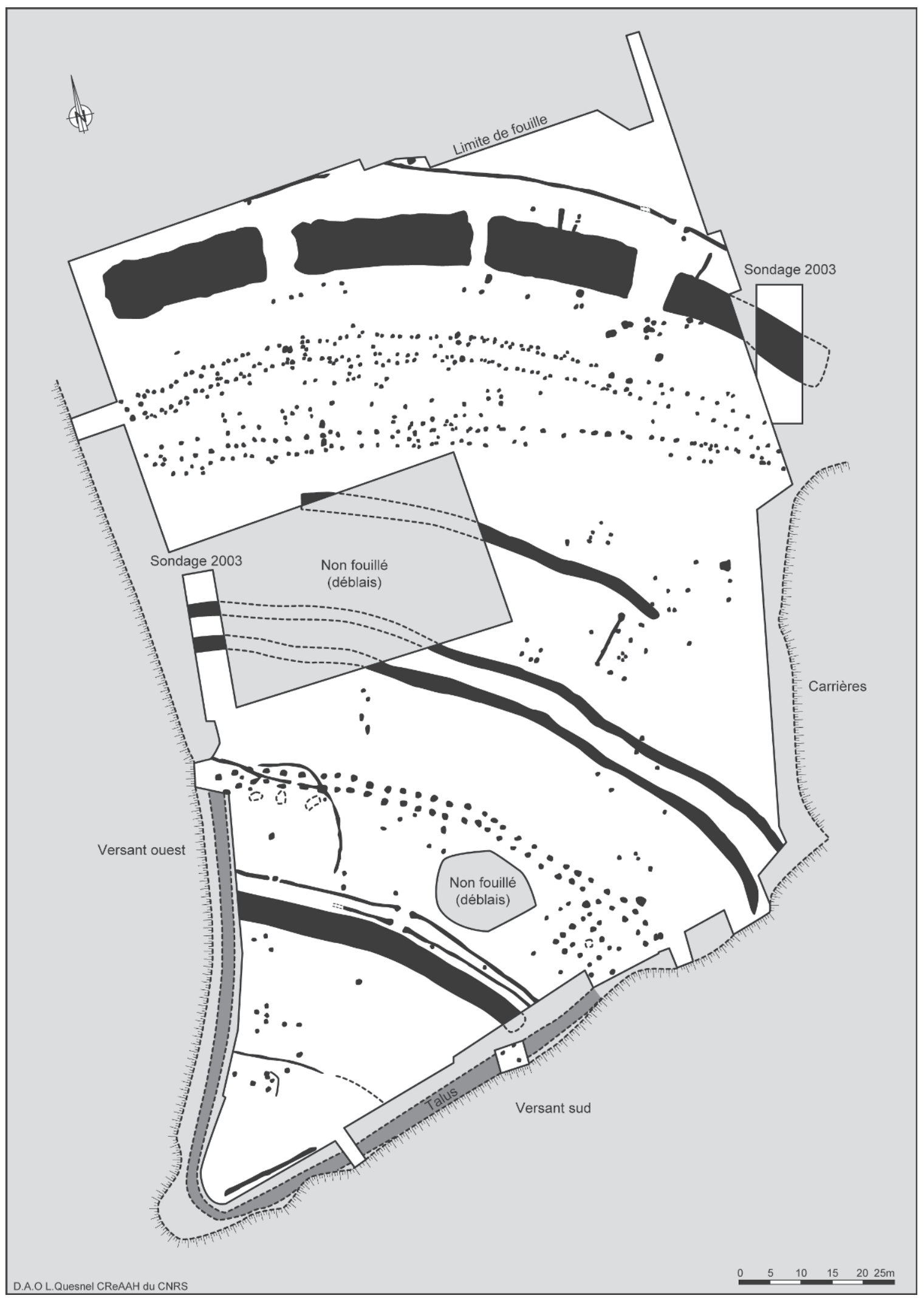

Figure 8 : Plan d'ensemble des structures archéologiques, du Néolithique ancien au Moyen Âge. Figure 8: General plan of archeological structures, from early Neolithic to Middle Ages. 
de leur succession chronologique qui, par ailleurs, reflète peu ou prou leur répartition spatiale du nord au sud, du plateau vers la pointe du promontoire. Ainsi, les trois principales occupations ayant laissé une forte empreinte sous la forme d'un système de barrage - à l'âge du Bronze, à l'âge du Fer et au haut Moyen Âge - montrent une réduction progressive de l'emprise enclose vers la pointe du promontoire. Nous distinguerons ainsi successivement sept ensembles principaux (fig. 4) :

- cinq foyers, dont trois sont datés du Néolithique ancien, sont regroupés dans l'ensemble 1 ;

- les structures parallèles du premier barrage, daté de l'âge du Bronze, constituant l'ensemble 2 ( $2 \mathrm{a}$ à $2 \mathrm{~d}$ );

- l'ensemble 3, qui associe la palissade du premier âge du Fer et ses structures annexes;

- la zone centrale qui regroupe dans l'ensemble 4 les trois fossés parallèles $(4 \mathrm{a}-4 \mathrm{c})$, le plan d'un bâtiment (4d) et les petits ensembles de trous de poteau $4 \mathrm{e}$ et $4 \mathrm{f}$;

- le grand fossé sud, daté de l'âge du Fer et défini comme ensemble 5;

- l'ensemble 6 est représenté par l'enceinte couvrant la pointe du promontoire (6a-6b) et le bâtiment associé (6c), datés du haut Moyen Âge;

- enfin, les structures plus légères du bas Moyen Âge sont regroupées en $7 \mathrm{a}$ à $7 \mathrm{~d}$.

\section{Quelques Vestiges du NéolithiQue ANCIEN (J.-Y. T., J. V. D. P., C. O.)}

Dans le secteur nord-est du site, en bordure du grand fossé segmenté, cinq anomalies se distinguent des trous de poteau habituels par leur morphologie et leur comblement (fig. 4, 9, 10 ; structures fo. $01,02,03,05$ et 06 ). La fouille en quadrants y a mis en évidence des traces de combustion par la présence de blocs de schiste chauffés et disposés à plat, plus rarement de galets de quartz, reposant sur un lit de cendres à très rares charbons de bois. Ces blocs, fréquemment fissurés et éclatés sous l'action de la chaleur, sont contenus dans une matrice fine de sédiments rougeâtres parfois mélangés de cendre. Ces éléments sont conservés dans des cuvettes circulaires ou ovalaires creusées dans le schiste et aux parois rubéfiées. Les dimensions varient en diamètre de $1,10 \mathrm{~m}$ à $1,40 \mathrm{~m}$ et leur profondeur va de 0,10 à $0,25 \mathrm{~m}$ en partie centrale. En restituant une épaisseur de 0,40 à $0,50 \mathrm{~m}$ de terre végétale, ces structures en fosses pouvaient atteindre 1,50 à $2 \mathrm{~m}$ de diamètre et 0,60 à $0,80 \mathrm{~m}$ de profondeur. Malgré un tamisage exhaustif des contenus, le mobilier en est absent à l'exception d'un tesson sans particularité mis au jour en fo.05.
La fouille a mis en évidence une chronologie relative (fig. 4 et infra, fig. 20), fo.02 étant recoupée par le trou de poteau 369 , montant de l'une des portes d'accès du système de barrage ceinturant la partie nord du site dont l'étude sera détaillée dans le chapitre suivant. Par ailleurs, ces cinq structures de combustion sont regroupées en bordure interne des tronçons orientaux $2 \mathrm{~b} .3$ et $2 \mathrm{~b} .4$ du fossé segmenté à l'emplacement du rempart adjacent (cf. infra, fig. 16); les foyers en cuvette ont ainsi été scellés par le système de barrage.

En prévision d'une recherche sur la fonction de ces structures de combustion, à partir d'analyses chimiques notamment, en collaboration avec l'UMR 6566, une série de prélèvements minutieusement positionnés a été réalisée en novembre 2005 sur fo.01, fo.03, fo. 05 et fo.06; cette opération est malheureusement restée sans suite. En revanche, les rares charbons de bois ont permis d'obtenir, pour trois des foyers, trois datations radiocarbone par accélérateur dont les résultats s'accordent sur le premier tiers du Ve millénaire avant J. C. (sur l'ensemble du site, les datations sont données à deux sigma) :

- fo.03 : Lyon-4661 (SacA-9812) : $5990 \pm 35$ BP, soit 4978-4791 av. J.-C. en date calibrée;

- fo.05 : GrN-30615: $6000 \pm 30$ BP, soit 4950-4782 av. J.-C. en date calibrée;

- fo.06 : GrN-30616 : $5860 \pm 40$ BP, soit 4810-4600 av. J.-C. en date calibrée.

L'étude anthracologique des charbons du foyer fo.06 (voir infra, $\$$ XII B1) montre une prédominance du chêne, accompagné de Pomoïdées. Les contraintes de croissance sur les cernes du chêne indiquent un couvert forestier dense, en milieu pauvre et sec signalé par la présence de petits fruitiers, probablement localisée sur le promontoire lui-même.

Ces cinq structures aux caractéristiques similaires et aux datations homogènes ne semblent associées à aucun autre élément. Un seul tesson érodé provient du foyer fo.05. De couleur brun clair à beige intérieurement comme extérieurement avec une tranche noire, il s'agit d'un fragment $(3 \mathrm{x}$ $3 \mathrm{~cm}$ ) de panse d'un grand vase modelé (10-12 mm d'épaisseur et large courbure de la paroi). Les traces d'un lissage sommaire sont visibles sur la surface externe craquelée et érodée. Le dégraissant abondant est majoritairement constitué de grains de quartz roulés avec quelques fragments de roche plus volumineux (jusqu'à $5 \mathrm{~mm}$ ). La cuisson est de bonne qualité.

Provenant du fond de fossé $2 \mathrm{~b} 4 \mathrm{du}$ Bronze final, un second tesson peut être associé à cette première occupation par son aspect. Il s'agit d'un bord de vase modelé à pâte gris noir, lèvre simple et décor léger de deux coups d'ongle. Par ailleurs, quelques pièces lithiques, isolées ou piégées dans les aménagements plus récents, pourraient être contemporaines de cette première occupation (cf. infra, fig. $22 \mathrm{n}^{\circ} 1$ et fig. 24) 
Figure 9 : Les structures de combustion en cuvette fo.01 et fo.03, du Néolithique ancien, en cours de fouille.

Figure 9: View of basin hearths fo.01 and fo.03 (Early Neolithic), under excavation.

Bien qu'isolées de tout contexte, ces structures de combustion constituent un nouveau jalon sur la route des premiers agriculteurs de notre région dont les témoignages se multiplient peu à peu à la suite de la découverte en 1995 de la maison VSG du Haut-Mée à Saint-Étienne-en-Coglès (Cassen et al., 1998). Plusieurs datations radiocarbone provenant de ce dernier site sont comparables à celles des foyers de La Rochette et la présence d'un amas de quartz rubéfiés au centre du bâtiment y évoque les vestiges résiduels d'un foyer. Depuis, des références à la première moitié du Ve millénaire ont été attestées jusqu’à la pointe occidentale de la Péninsule bretonne. Au nord du bassin de Rennes, la fouille préventive en 2004 du site de Pluvignon à Betton atteste de la présence du VSG dès son étape moyenne, d'après certains décors de la céramique et les datations (Blanchet $e t$ al., 2007). Plus à l'ouest, des prospections dans la moyenne vallée du Blavet mettent en évidence un matériel lithique caractéristique du Néolithique ancien, associant des anneaux en schiste, des lames en silex d'importation, des armatures asymétriques de type danubien (Pailler et al., 2008). Enfin ce courant de néolithisation ancien est reconnu jusqu'aux pointes de la Péninsule grâce aux résultats de prospections dans le secteur de Brest (ibid.), à la présence d'un foyer
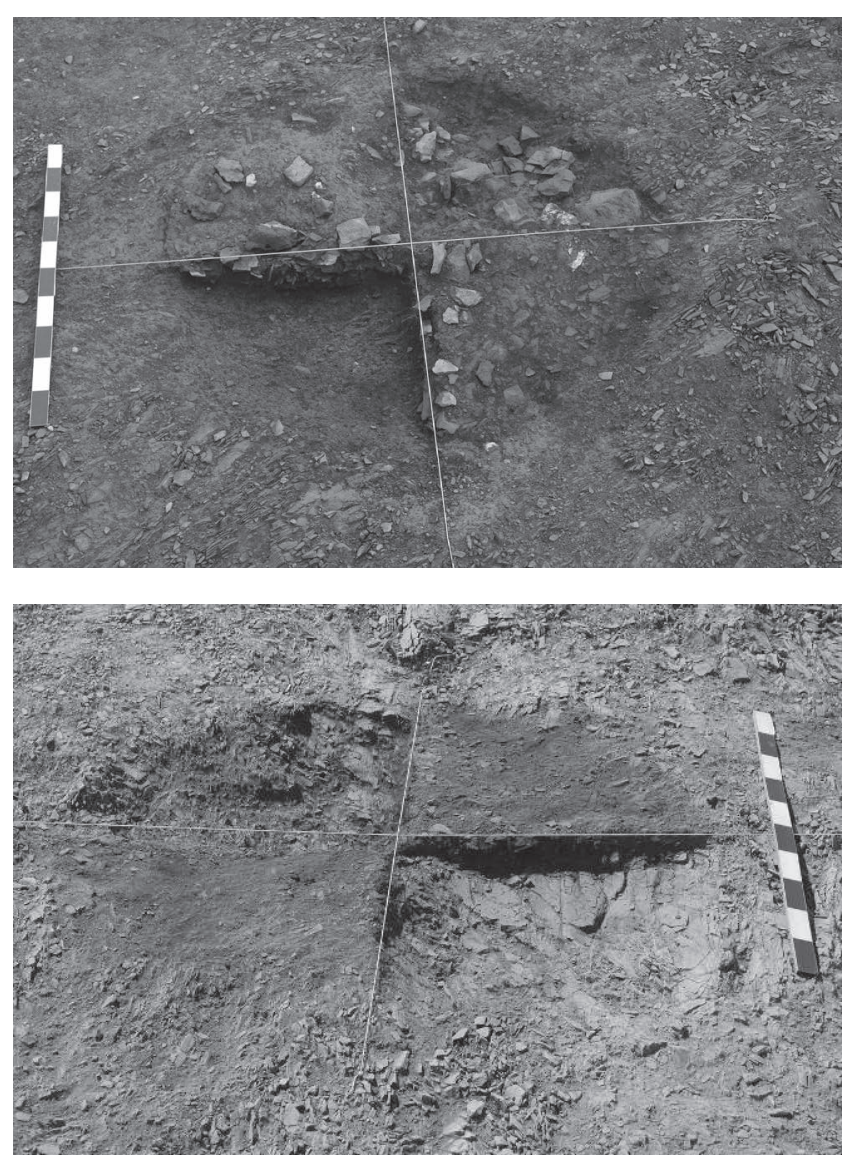

daté sous l'un des monuments du complexe mégalithique du Souc'h à Plouhinec (Finistère) (Le Goffic, 2009) et à la découverte de fosses d'habitat contenant du mobilier VSG à Quimper, lieu-dit Kervouyec-Nevez (Tinévez et al., 2006).

$\mathrm{Du}$ grand fossé $2 \mathrm{~b}$ de La Rochette provient également un bord de vase à lèvre plate et bandeau de décor oblique caractéristique du Campaniforme (cf. infra, fig. 22, no 2); il témoigne d'une fréquentation du site à la fin du IIIe millénaire. Son décor d'impressions légères obliques semble réalisé à la coquille et est limité par de fines incisions horizontales.

\section{UNE OCCUPATION IMPORTANTE ET TRÈS STRUCTURÉE DE L'ÂGE DU BRONZE (J.-Y. T., M. F.)}

Le second ensemble constitue un important barrage à l'accès nord du site et est l'expression d'une occupation massive du promontoire (fig. 4 et 11 à 20). Ce système, au plan curviligne très régulier orienté est-ouest, est axé sur le grand fossé à l'origine de l'opération de fouille. Il est implanté dans une légère dépression du terrain où le schiste est particuliè- 
Figure 10 : Ensemble 1 : cinq structures de combustion du Néolithique ancien au nord-est du site; position et datations 14C, plans de détail et coupes.

Figure 10: Whole 1: five early Neolithic hearths on northeast of the site: location and $14 \mathrm{C}$ datings, detail plans and sections.

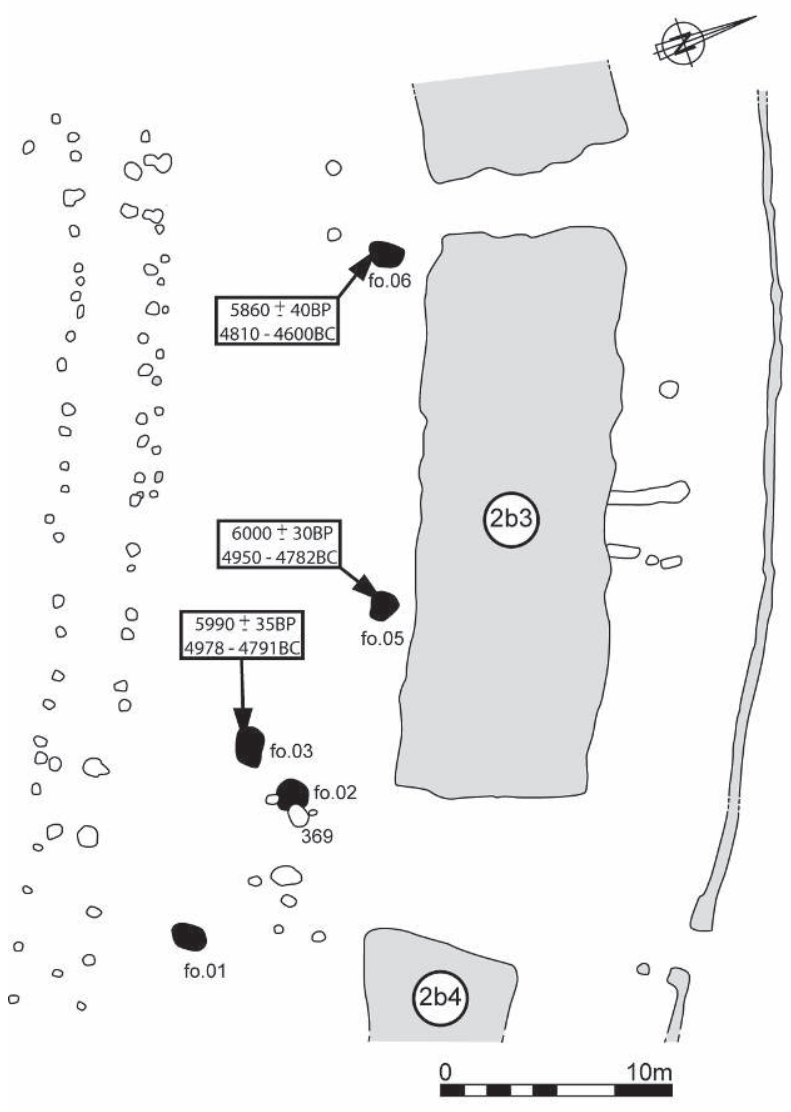

D.A.O L.Quesnel CReAAH du CNRS

rement altéré et se transforme progressivement en substrat argileux jaune clair dans la partie orientale. La nature plus fine de l'encaissant dans le secteur nord-est conditionne naturellement l'aspect plus limoneux des comblements de fondations qui sont ainsi nettement moins contrastées. Par ailleurs, ce secteur est traversé en diagonale du nord-ouest au sud-est par une bande étroite de limon jaune clair très compact et parsemé de petits galets de quartz roulés. Trois
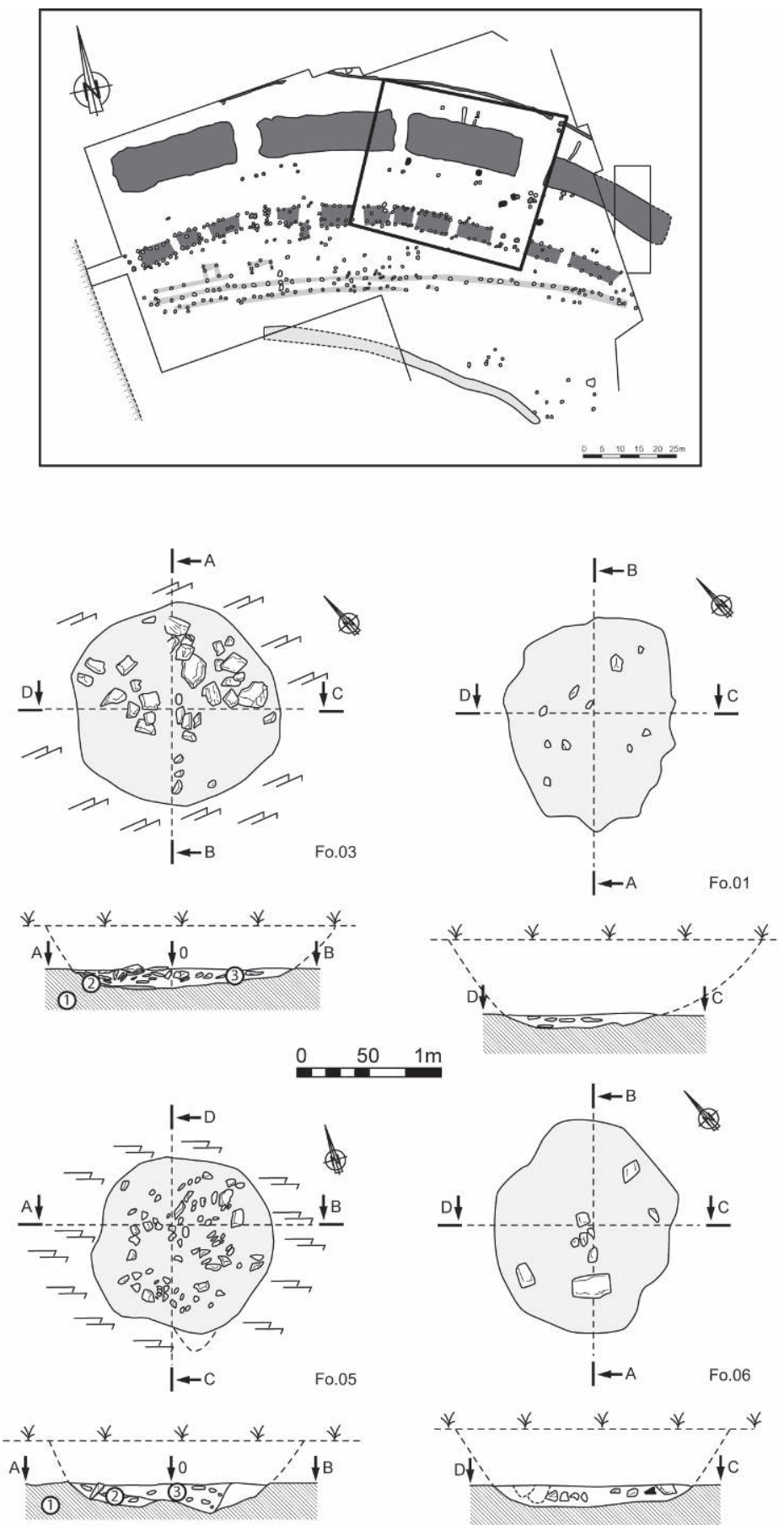

types de structures en creux se succèdent du nord au sud à intervalle régulier et frappent par leur parallélisme quasi parfait : une palissade continue $2 \mathrm{a}$, les quatre tronçons du fossé $2 b$, un espace presque vierge de vestiges $2 c$ et un ensemble structuré de trous de poteau $2 \mathrm{~d}$. 
Figure 11 : Vue globale à partir de l'ouest de l'ensemble de l'âge du Bronze (aires fouillées en 2004 et 2005); à droite, palissade et structures du premier âge du Fer.

Figure 11: General view from the west of the Bronze age structures excavated in 2004-2005; on the right, Iron Age palissade and structures.

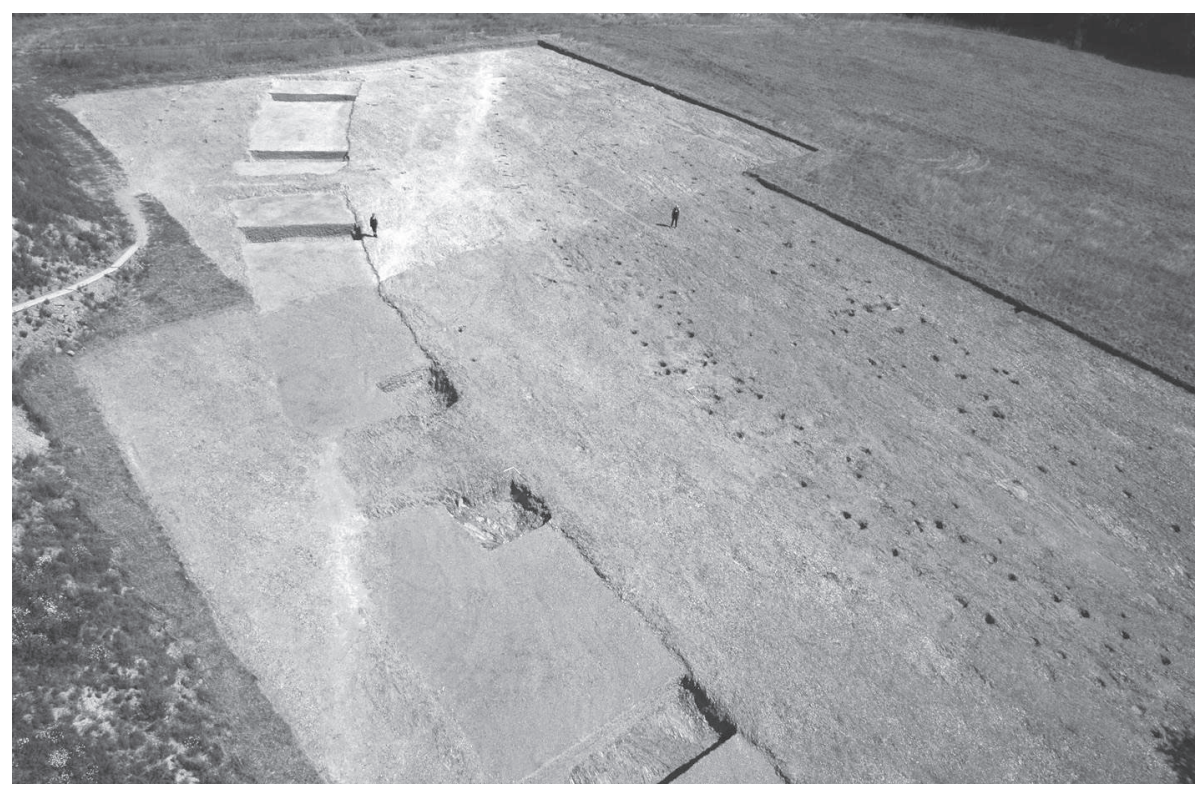

\section{A. Une première palissade au nord (structure 2a)}

Depuis le plateau vers le promontoire, un premier obstacle est matérialisé par la tranchée étroite d'une palissade interrompue à deux reprises, au nord et à l'est. Cette tranchée de 0,25 à $0,50 \mathrm{~m}$ de large pour 0,10 à $0,25 \mathrm{~m}$ de creusement dans le schiste (soit une fondation initiale pouvant être estimée entre 0,60 et $0,80 \mathrm{~m}$ de profondeur) est reconnue sur une longueur de $80 \mathrm{~m}$ en quatre tronçons distincts, $2 \mathrm{a} 1 \mathrm{à}$ 2a4 (fig. 12, 15, 16). La tranchée, à fond plat et profil en $\mathrm{U}$, est comblée d'un sédiment ocre jaune incluant quelques petites plaquettes de schiste et quelques petits tessons, mais sans éléments de calage; cependant, par endroits, l'élargissement des bords et de légères différences de couleur et de texture trahissent l'emplacement de poteaux. Malgré l'arasement de son extrémité occidentale, on peut estimer à plus d'une centaine de mètres sa longueur d'origine. Elle rejoignait ainsi les deux flancs du promontoire. Le tracé, d'apparence curviligne à première vue, est en réalité constitué d'une suite de tronçons rectilignes, parallèles au grand fossé et les ruptures d'orientation se situent à hauteur de l'axe médian des éléments $2 \mathrm{~b} .2$ et $2 \mathrm{~b} .3$ du fossé. L'hypothèse d'une première palissade relativement légère comme élément marqueur de l'espace enclos peut être proposée. L'entrée orientale, large de $1,50 \mathrm{~m}$, est bordée de deux forts trous de poteau, TP. 500 et 501 , de $0,70 \mathrm{~m}$ de diamètre et $0,35 \mathrm{~m}$ de profondeur conservée, contenant chacun la trace de deux poteaux jointifs (diamètres 0,25 et $0,15 \mathrm{~m}$ ). À un mètre en retrait de 501 , TP.502 $(0,50 \mathrm{~m}$ de diamètre et $0,30 \mathrm{~m}$ de profondeur) et la tranchée $2 \mathrm{a} .4$, perpendiculaire et fai- blement marquée dans le substrat, complètent l'aménagement de ce secteur (fig. 20). Une seconde interruption de la palissade, nettement plus large $(9 \mathrm{~m})$, est visible au droit du fossé $2 \mathrm{~b} .2$ et est bordée de légers trous de poteau, ce qui semble exclure un arasement de la structure à cet endroit. En revanche, son extrémité ouest, en limite de fouille, n'a pu être déterminée.

Un espace large de $6 \mathrm{~m}$, quasiment vide de vestiges, sépare cette première palissade du grand fossé. On y notera toutefois la présence de petites tranchées perpendiculaires au fossé $2 \mathrm{~b} .3$, proches en morphologie de $2 \mathrm{a} .4$ mais d'autant plus difficiles à interpréter que ce secteur est fortement perturbé par des chablis anciens.

\section{B. Un large fossé segmenté en quatre éléments}

Les quatre tronçons qui constituent cette large excavation ont été reconnus au niveau du substrat sur la quasi totalité de leur ouverture, à l'exception de l'extrémité orientale de 2 b.4 (fig. 8, 13, 14). Ils ont fait l'objet de neuf sondages au total (soit $17 \%$ environ du volume du comblement). Les dimensions mettent en évidence la régularité des proportions de chaque élément (tabl. 1).

Les sondages (six transversaux et trois sondages d'angle dont deux de part et d'autre de l'interruption 2b1-2b2) fournissent des informations homogènes sur la morphologie et le processus de comblement (fig. 13). Le fossé est à fond plat et flancs abrupts. La profondeur dans la masse du schiste varie de $0,80 \mathrm{~m}$ à $1,20 \mathrm{~m}$ et, si on ajoute une épaisseur moyenne de terre végétale de $0,50 \mathrm{~m}$, l'estimation de l'excavation peut être comprise entre 1,30 et 1,70 m. 


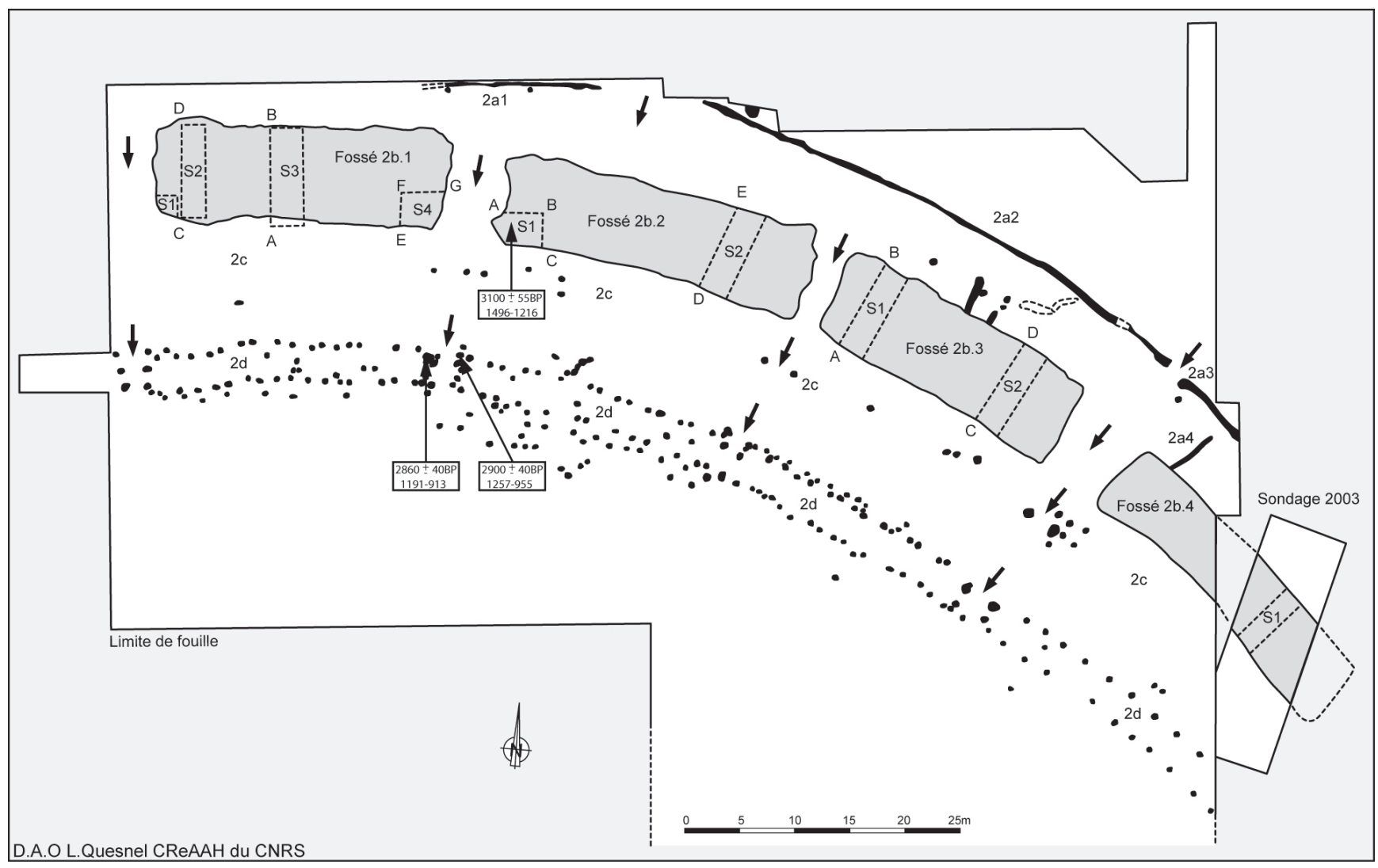

Figure 12: Plan d'ensemble des structures de l'âge du Bronze au nord du site.

Figure 12: General plan of the Bronze age structures, north of the site.

Les différentes coupes révèlent une dynamique de comblement très similaire d'un sondage à l'autre (fig. 13 et 14).

Dans un premier temps, le processus de dégradation des bords se traduit par une accumulation de plaquettes de schiste de bonne taille au pied des parois. Ces niveaux sont caractérisés par une quasi absence de terre interstitielle entre les plaquettes dont l'orientation et l'obliquité trahissent un effondrement brutal de blocs de schiste sans remaniement ultérieur (US.6, 7 et 8 des coupes; fig. 13). Un échantillon de charbon de bois piégé par l'un de ces effondrements correspondant à une phase précoce du comblement, dans l'angle sud-ouest du fossé $2 \mathrm{~b} .2$ (sondage $\mathrm{S} 1$ ), donne la datation Gif-12111: $3100 \pm 55$ BP, soit 1496-1 216 av. J.-C. en âge calibré.

Dans un deuxième temps (US. 3 à 6), le pendage nettement oblique des couches indique un comblement progressif du fossé à partir de son bord interne. L'alternance de niveaux exclusivement formés de plaquettes de schiste et de niveaux à matrice plus fine et terreuse évoque l'effondrement d'une masse de remblais accumulés sur le bord interne du fossé. En revanche, les coupes perpendiculaires aux extrémités des fossés de part et d'autre d'une interruption ne révèlent pas les mêmes pendages, sans doute en l'absence de dépôts de déblais sur l'emprise du passage. Tous les sondages transversaux montrent une certaine dissymétrie dans le profil des flancs et une dégradation accentuée du bord externe; cette caractéristique pourrait résulter d'une meilleure préservation du bord interne grâce à la protection d'un talus sus-jacent et de son éboulement progressif.

Dans un troisième temps (US. 1 et 2), les niveaux supérieurs, à profil en berceau, sont de nature plus homogène et expriment un comblement massif et plus récent de la dépression, longtemps préservée après que le système talus-fossé ait atteint son profil d'équilibre. D'après la nature des terres et les quelques objets métalliques retrouvés, cette dernière étape semble correspondre aux travaux de remembrement agricole des années soixante.

Les tronçons sont séparés d'interruptions d'une largeur variant de 3 à $6 \mathrm{~m}$ et laissant un passage d'une dizaine de mètres en bordures est et ouest du promontoire. Ces passages ne laissent apparaître aucun aménagement particulier. 
Figure 13 : Coupes du fossé segmenté $2 \mathrm{~b}$.

Figure 13: Sections of the segmented ditch $2 b$.

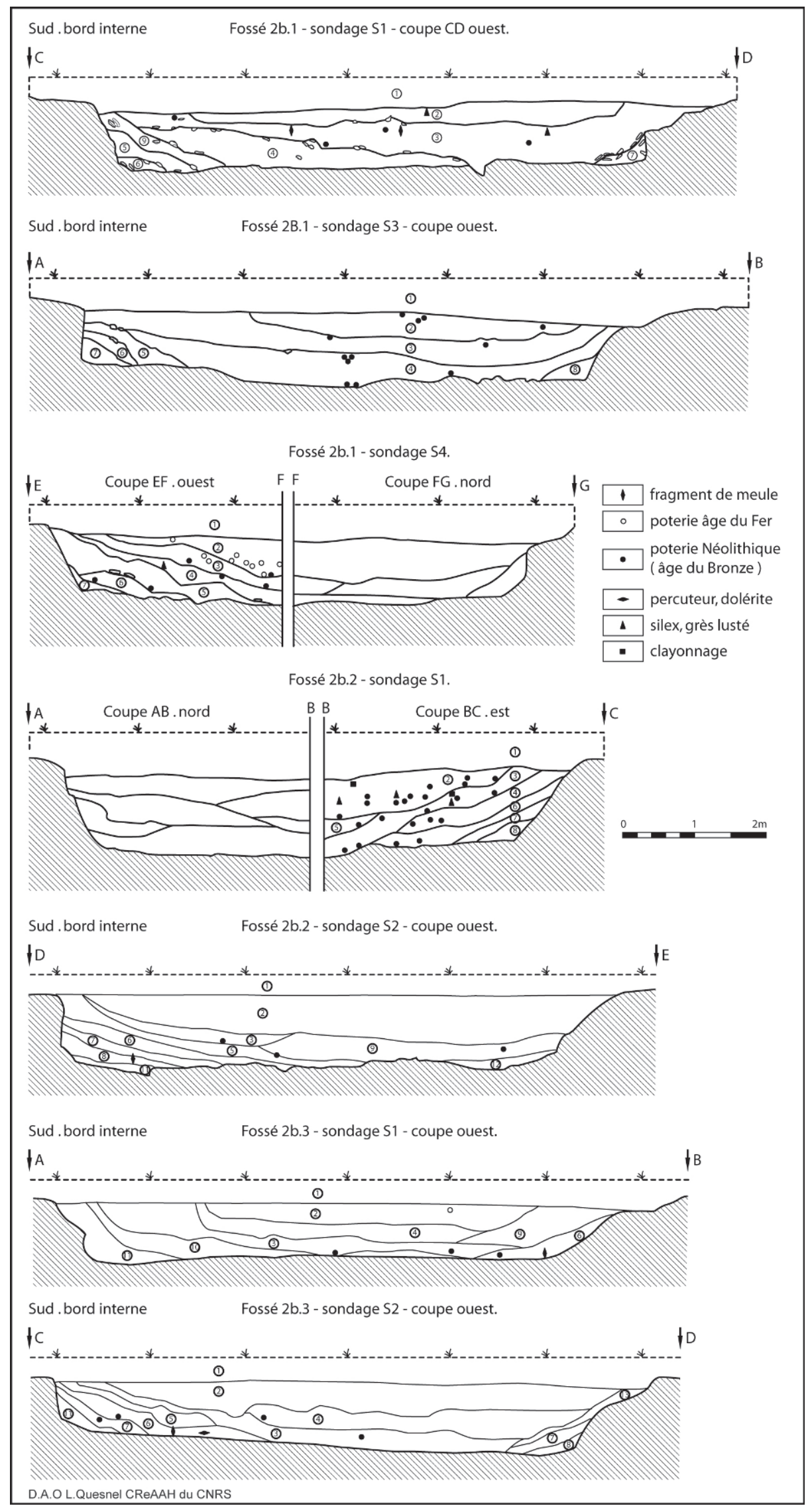

Sud . bord interne Fossé $2 \mathrm{~b} .2$ - sondage $\mathrm{S} 2$ - coupe ouest.

D

Sud . bord interne Fossé $2 \mathrm{~b} .3$ - sondage $\mathrm{S} 1$ - coupe ouest. 

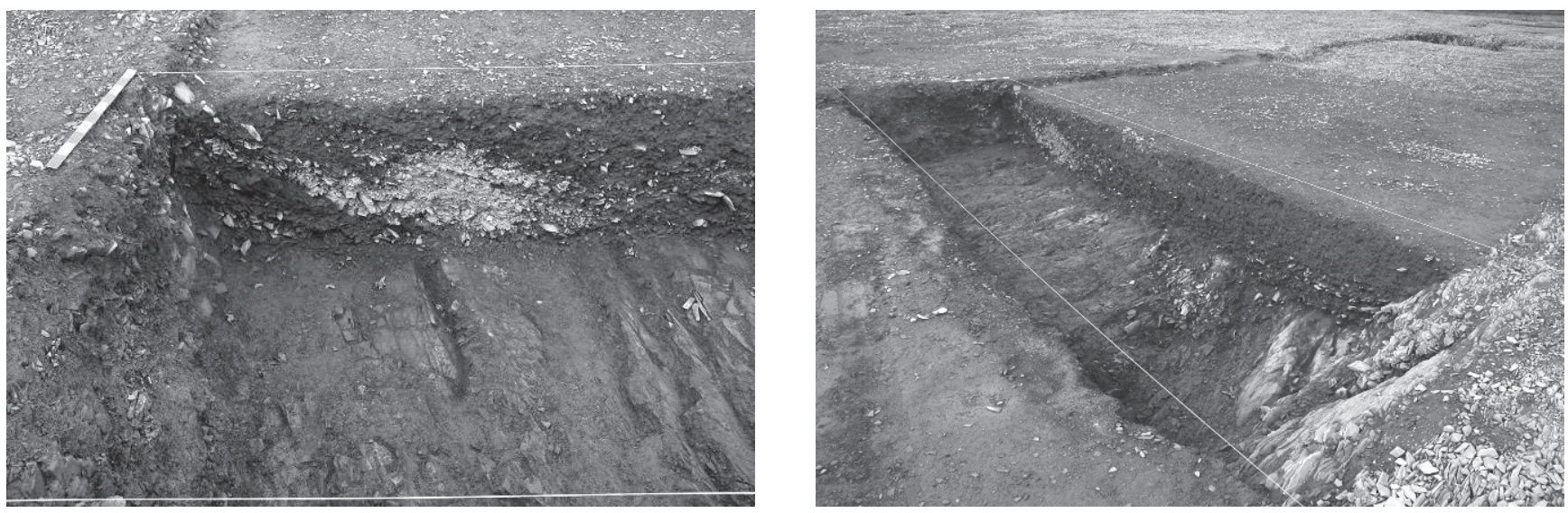

Figure 14 : Sondage transversal $\mathrm{n}^{\circ} 2$ dans le fossé $2 \mathrm{~b} .2$ et détail de la coupe ouest, le côté interne de l'enceinte montrant les effondrements successifs du rempart.

Figure 14: Transversal sondage $n^{\circ} 2$ in the 26.2 ditch and detail of west section (internal edge), showing successive collapses of the rampart.

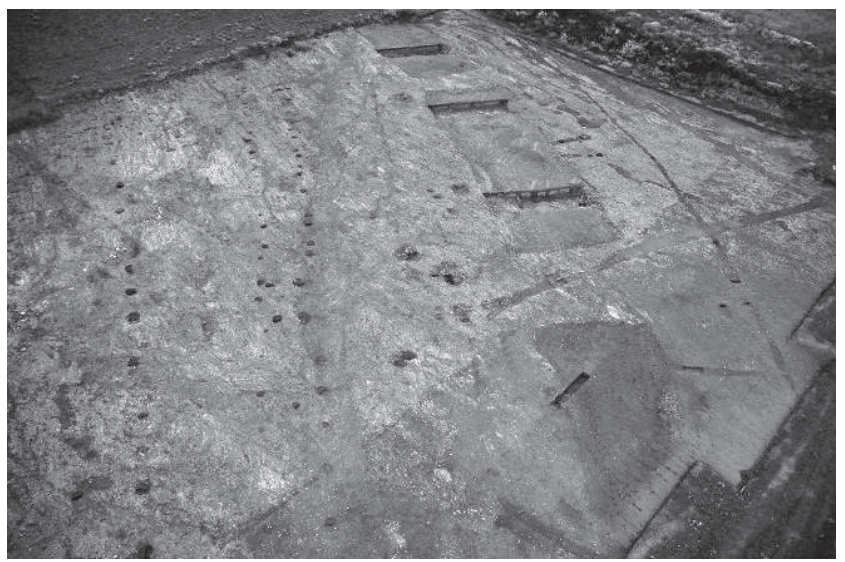

Figure 15 : Vue globale depuis l'est de l'ensemble du Bronze et de la palissade du premier âge du Fer (à gauche; aire fouillée en 2005 et 2006 au premier plan).

Figure 15: General view from the east of the Bronze age settlement and of the first Iron Age palisade (on the left; 2005 and 2006 digging areas on foreground).

\section{C. L'assise d'un rempart arasé (ensemble 2c)}

La structure excavée précédente est bordée côté interne d'un nouvel espace quasiment vierge de vestiges et large de 10 à $11 \mathrm{~m}$. Hormis un couple de poteaux bien localisé en vis-à-vis de chaque interruption du fossé, les seules structures mises au jour au nord-est de cet espace sont les foyers datés du Néolithique ancien précédemment décrits. L'analyse du comblement du grand fossé et l'absence de structures contemporaines dans cet espace permettent de l'interpréter comme assise du rempart édifié avec les déblais de creusement.

\begin{tabular}{|c|c|c|c|}
\hline tronçon & longueur & largeur & profondeur \\
\hline $2 \mathrm{~b} .1$ & $27 \mathrm{~m}$ & 8 à $9 \mathrm{~m}$ & 1,00 à $1,20 \mathrm{~m}$ \\
\hline $2 \mathrm{~b} .2$ & $29 \mathrm{~m}$ & $8 \mathrm{~m}$ & 0,90 à $1,20 \mathrm{~m}$ \\
\hline $2 \mathrm{~b} .3$ & $24 \mathrm{~m}$ & $8 \mathrm{~m}$ & 0,80 à $1,20 \mathrm{~m}$ \\
\hline
\end{tabular}

Tableau 1 : Dimensions relevées sur le fossé segmenté $2 \mathrm{~b}$. Table 1: Dimensions noted on segmented trench $2 b$.

En prenant comme exemple le tronçon occidental $2 \mathrm{~b} .1$ de ce fossé, il est possible d'évaluer de façon assez précise le volume de déblais extrait de chaque tronçon. La surface excavée en surface du substrat y est de $27 \times 9 \mathrm{~m}=234 \mathrm{~m}^{2} \mathrm{et}$ celle du fond de fossé de $25 \times 7 \mathrm{~m}=175 \mathrm{~m}^{2}$. Avec une profondeur moyenne de $1 \mathrm{~m}$, le volume de schiste extrait atteint $208 \mathrm{~m}^{3}$. En ajoutant le volume de terre végétale avec une épaisseur estimée à $0,50 \mathrm{~m}\left(243 \mathrm{~m}^{2} \times 0,50 \mathrm{~m}=121,50 \mathrm{~m}^{3}\right)$, le volume total extrait peut être arrondi à $330 \mathrm{~m}^{3}$. Ainsi, compte tenu du foisonnement puis du tassement des déblais, estimé à $15 \%$, le volume de matériaux disponible peut être évalué à $380 \mathrm{~m}^{3}$ pour chaque tronçon.

Plusieurs hypothèses de réutilisation peuvent être proposées en fonction des données de la fouille. L'édification d'un talus sur la longueur totale du tronçon $(27 \mathrm{~m})$, sur une base de $6 \mathrm{~m}$ de large $\left(162 \mathrm{~m}^{2}\right)$ se réduisant à $3 \mathrm{~m}$ de large au sommet $\left(24 \times 3 \mathrm{~m}=72 \mathrm{~m}^{2}\right)$ permet d'atteindre une élévation de 3,20 m. Le dénivelé fossé/talus ainsi obtenu est de 4,50 à $5 \mathrm{~m}$. En revanche, le choix d'aménager une plate-forme surélevée jusqu'à la première série de poteaux à environ $10 \mathrm{~m}$ vers l'intérieur (soit sur une base de $270 \mathrm{~m}^{2}$ ) permet une élévation de 1,40 m et un dénivelé total d'environ $3 \mathrm{~m}$. Ce type d'aménagement aurait l'avantage d'assurer un meilleur ancrage au sol d'éventuelles superstructures défensives (palissades) sans avoir à creuser le substrat. 


\section{D. Une zone d'habitat adossée au rempart}

L'apport majeur des deux premières années de fouille est la mise au jour d'un nombre important de trous de poteau dont la répartition générale révèle une structuration de l'espace inédite en Bretagne pour ce type de site (fig. 12 et 16 à 19). Difficiles à repérer lors du décapage, ces structures se sont précisées au fur et à mesure du nettoyage minutieux du schiste, mais également à la faveur des variations du taux d'humidité du sol. Un total de 215 trous de poteau constitue cet ensemble $2 \mathrm{~d}$. Ils sont creusés dans un encaissant dont l'état varie d'ouest en est depuis un schiste bleuté très diaclasé se délitant en baguettes jusqu'à un substrat argileux altéré ocre jaune. La morphologie des fondations évolue en conséquence, depuis des trous aux bords anguleux, aux parois abruptes et au fond plat dans le premier cas jusqu’à des excavations plus circulaires au fond arrondi dans le second. La déclivité du terrain et l'épaisseur de la terre végétale s'accentuant vers l'est, la profondeur des fondations dans le substrat s'y amenuise progressivement et explique certainement quelques lacunes en zone orientale. Pour la majorité des individus, les dimensions se situent autour de 0,35 à $0,45 \mathrm{~m}$ de diamètre et 0,30 à $0,15 \mathrm{~m}$ de profondeur (soit une estimation entre 0,60 et $1 \mathrm{~m}$ de profondeur à l'origine). Toutes ces structures ont été fouillées par moitié dans un premier temps, puis, la plupart l'ont été entièrement avec tamisage à sec des comblements. La texture de ces derniers varie en fonction de l'état du substrat, d'une terre légère brun clair en partie ouest à un sédiment fin ocre jaune à l'est. Les pierres de calage sont quasi inexistantes et se réduisent à quelques blocs de schiste issus de l'extraction et à quelques blocs de quartz. La trace d'un poteau central, de $0,20 \mathrm{~m}$ de diamètre moyen, est parfois perceptible, $\mathrm{y}$ compris dans la zone orientale plus arasée. L'essentiel du rare

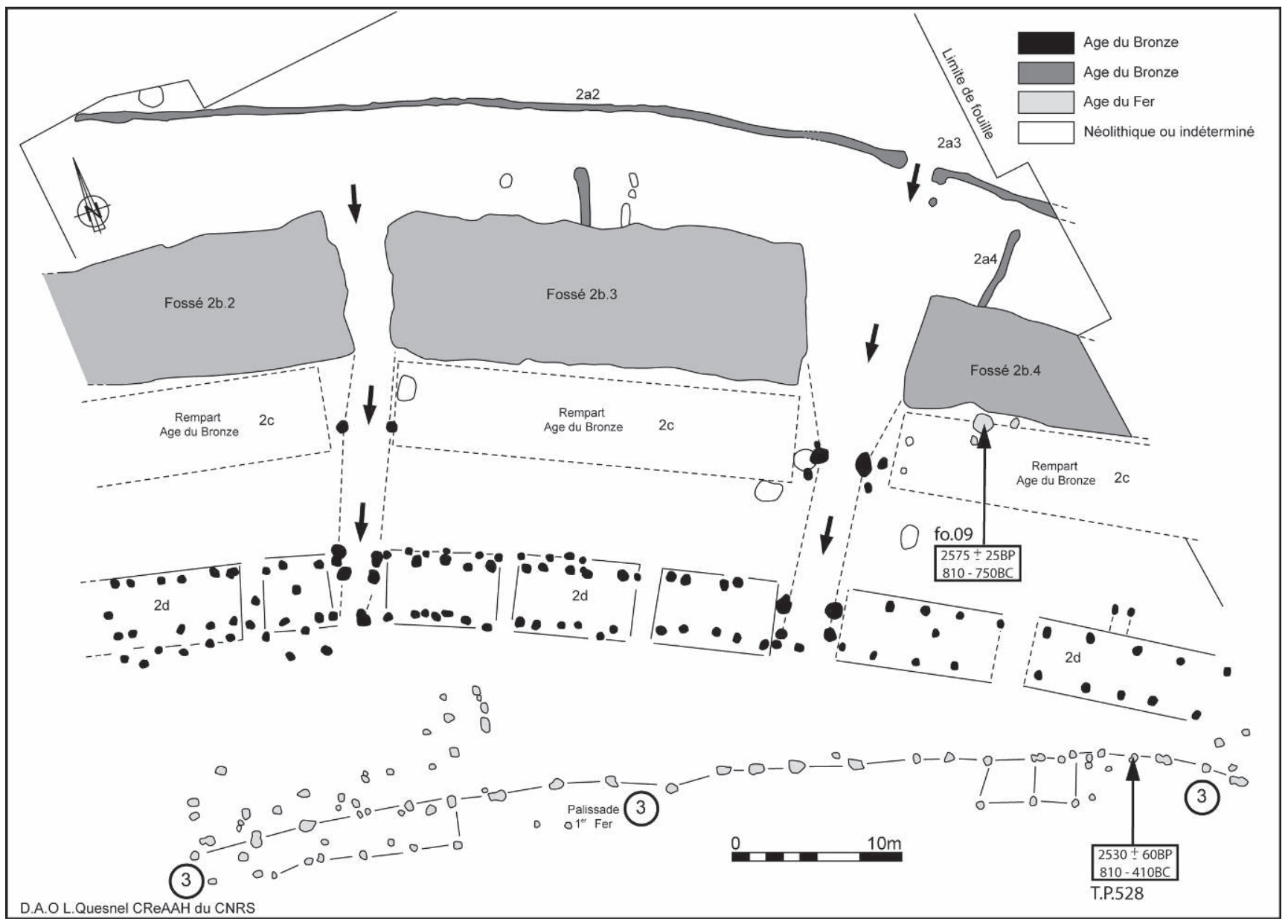

Figure 16 : Secteur est de l'habitat fortifié de l'âge du Bronze (ensemble 2a à 2d) et de la palissade du premier âge du Fer (ensemble 3). Le parallélisme remarquable entre les éléments de l'ensemble 2 n'est pas adopté par la palissade 3 plus récente.

Figure 16: East sector of the Bronze age fortified settlement (wholes $2 a$ to $2 d$ ) and of the first Iron age palisade (whole 3). The noteworthy parallelism of the whole 2 components is not adopted by the more recent palisade 3. 


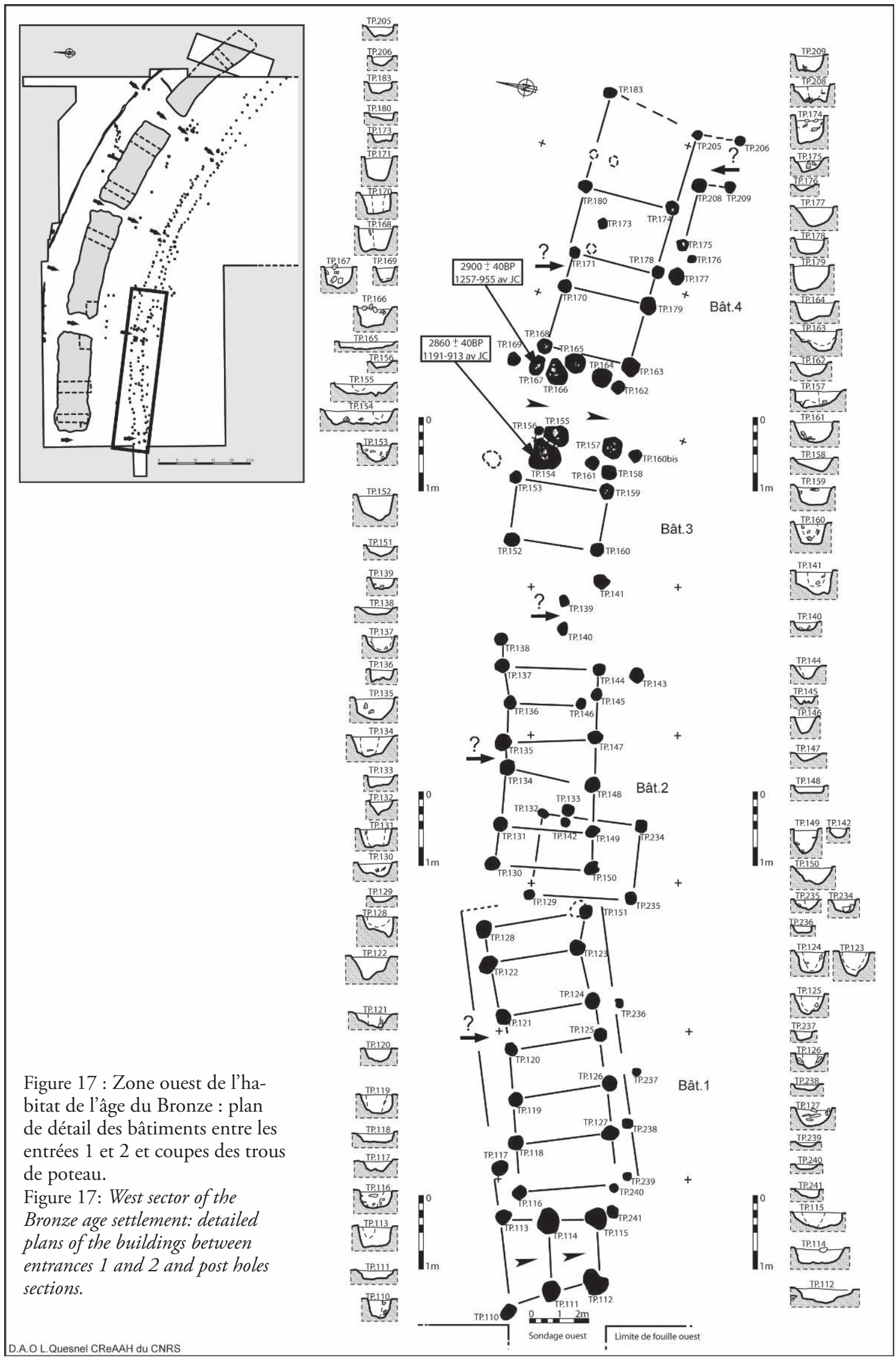




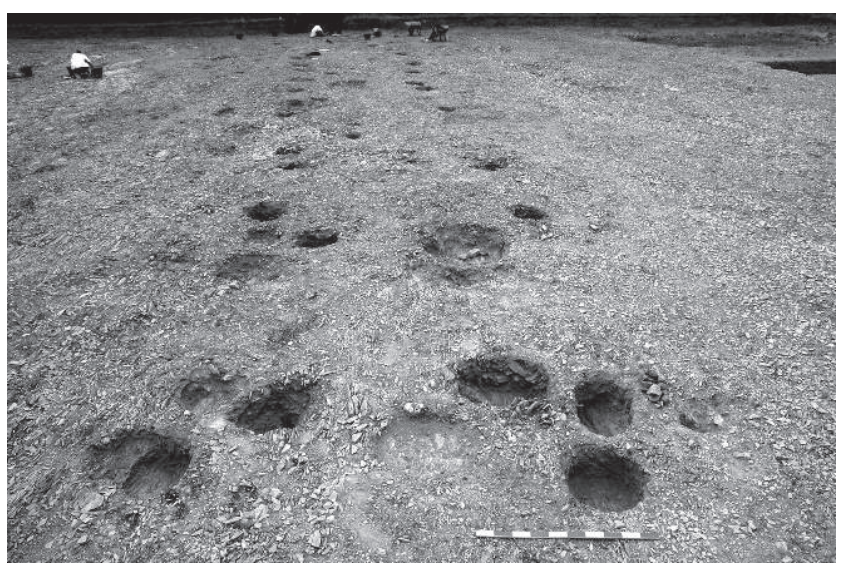

Figure 18 : Vue vers l'ouest des bâtiments du Bronze; première section ouest et fondations de la porte 2 au premier plan.

Figure 18: Westwards view of the bronze age buildings; first west section and gate 2 on foreground.

mobilier conservé - petits tessons, charbons, esquilles d'os calcinés - provient des trous de ce secteur (voir la répartition du mobilier fig. 21).

Le plan de cet ensemble est organisé sur deux lignes, séparées de $3 \mathrm{~m}$ l'une de l'autre, parfaitement parallèles entre elles et au grand fossé $2 \mathrm{~b}$, à une distance de 10-11 m de celui-ci. Dans une même ligne, les poteaux sont distants de $1 \mathrm{~m}$ à $1,50 \mathrm{~m}$ et certains semblent fonctionner par paires. Dans les secteurs ouest et central, les mieux conservés, ces deux séries sont partiellement doublées en extérieur par de petits trous alignés à $0,50 \mathrm{~m}$ environ des alignements précédents (fig. 16 et 17).

\section{D 1. Des accès multiples très structurés}

La cohérence de l'ensemble est renforcée par la présence de structures particulières en vis-à-vis de chaque interruption du grand fossé et séparant des lots de fondations homogènes (fig. 17 à 20). Elles matérialisent des entrées bâties sur un plan légèrement trapézoïdal, fondées, suivant les cas, sur quatre ou six trous, plus larges (0,70 à $0,90 \mathrm{~m}$ de diamètre) et moins profonds $(0,25 \mathrm{~m})$ que les précédents. L'hypothèse de porches monumentaux peut être ici évoquée. En vis-àvis de l'interruption entre $2 \mathrm{~b} .1$ et $2 \mathrm{~b} .2$, la porte correspondante, représentée par TP.154, 158, 167, 164 (fig. 17) a subi une réfection (TP.155, 157, 166, 162), à la suite d'un probable incendie d'après la présence d'une forte concentration de charbons, en TP.154 et 167 notamment. Ces lots de charbons donnent deux datations homogènes : TP.154: Gif-12112 : $2860 \pm 40 \mathrm{BP}$, soit 1 191-913 av. J.-C. en âge calibré et TP.167 : Gif-12113: $2900 \pm 40$ BP, soit 1257 955 av. J.-C.

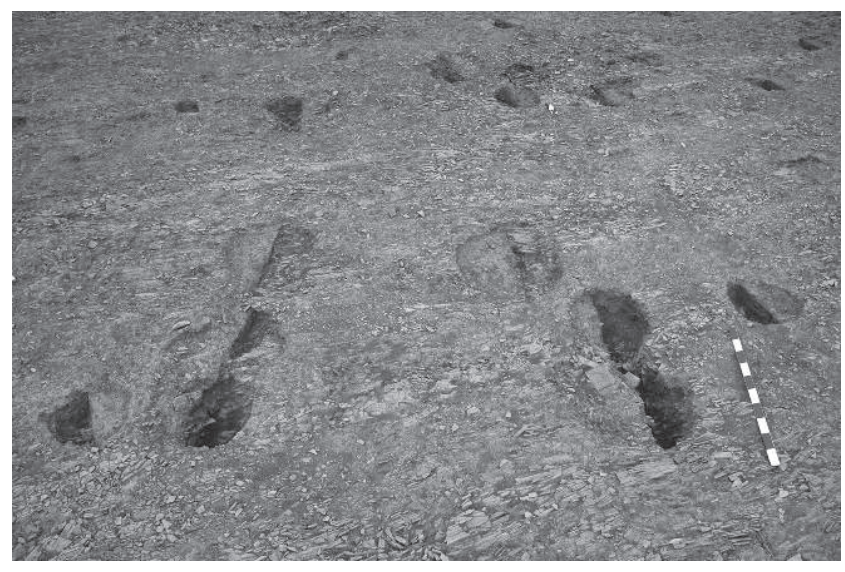

Figure 19 : Vue vers le sud de la porte 3 entre les sections 3 et 4 des bâtiments du Bronze.

Figure 19: Southwards view of gate 3 between sections 3 and 4 of the Bronze Age buildings.

Cette forte structuration des entrées est complétée par la présence de paires de poteaux en zone intermédiaire en bordure du rempart, à une distance égale de $4 \mathrm{~m}$ du bord interne du grand fossé $2 \mathrm{~b}$. En vis-à-vis de l'interruption entre $2 b .3$ et $2 b .4$, un véritable couloir d'accès vers la partie interne du promontoire est ainsi balisé dès la palissade nord (fig. 20) : première porte (TP.500-501), interruption 2b.-2b.4, seconde porte avec TP.330, 332, 369 et 387 (recoupant le foyer néolithique fo.02), troisième et dernière porte (TP.281, 281bis, 83bis et 334). Ce schéma régulier se répète à chaque interruption du fossé, sans toutefois de première ouverture dans la palissade nord.

Les charbons prélevés dans plusieurs trous, dont ceux des deux porches occidentaux ont fait l'objet d'étude anthracologique (voir infra, $\$$ XII B2). Le chêne y reste dominant, accompagné du genêt/ajonc, du noisetier et de l'érable. De calibre moyen, les fragments de chêne, dont la largeur de cernes est plus du double de celle observée au Néolithique ancien, traduisent une ouverture du milieu corroborée par les taxons du genêt/ajonc. La présence du noisetier et de l'érable, nécessitant un milieu plus riche, indique un approvisionnement en bois différent de celui du Néolithique, probablement plus éloigné du promontoire déjà largement défriché.

\section{D 2. Fonction de l'ensemble 2d: palissades ou habitats?}

Sur la fonction de cet ensemble de fondations parfaitement structuré, il vient à l'esprit une première hypothèse de deux palissades servant d'armature au rempart édifié en arrière du grand fossé. Plusieurs observations et arguments infirment cette première interprétation. Le processus de 


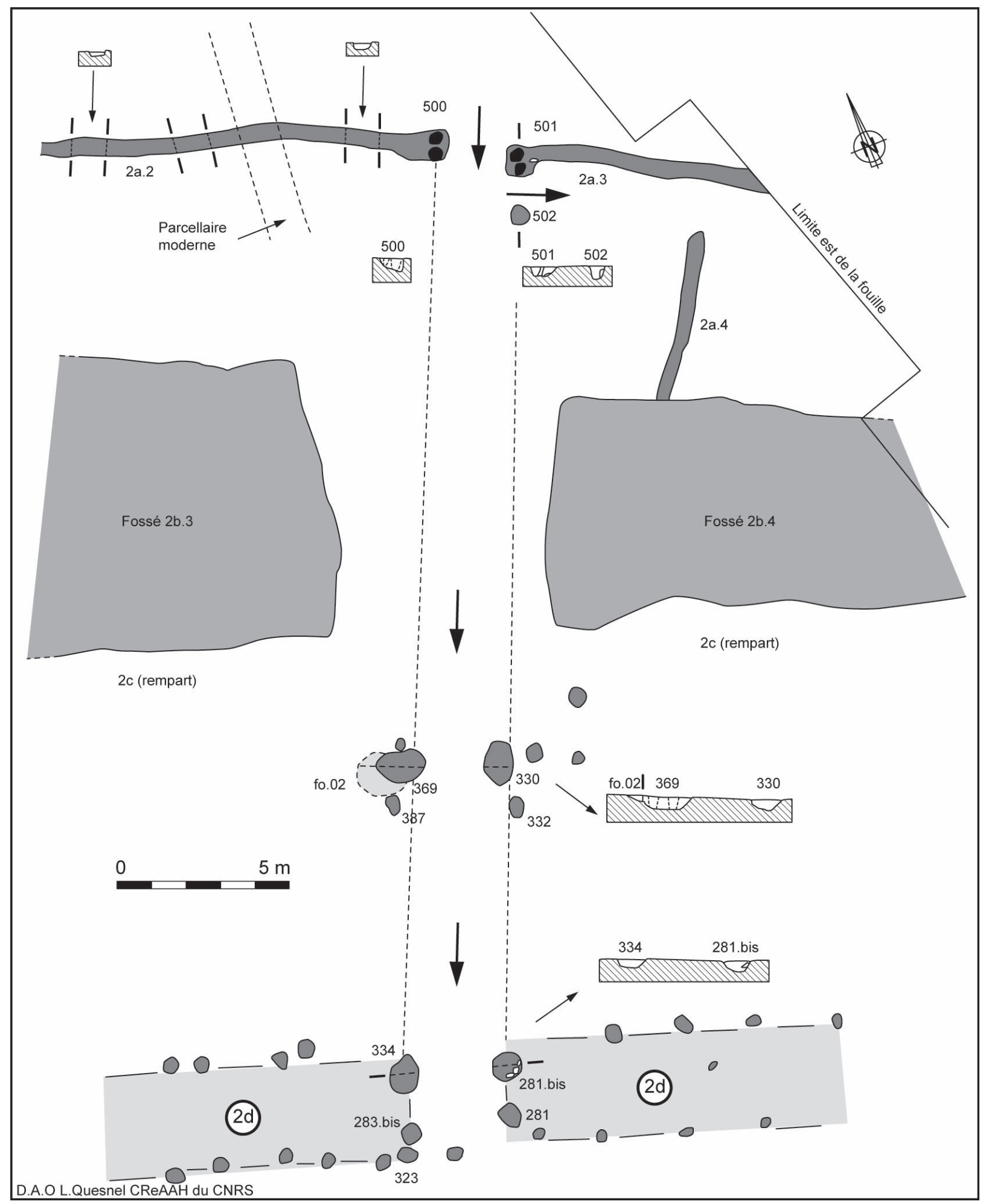

Figure 20 : Zone est de l'habitat fortifié de l'âge du Bronze : plan de détail du système d'accès $n^{\circ} 4$ entre la palissade $2 a$ et les bâtiments $2 \mathrm{~d}$. Le trou de poteau 369 de la porte intermédiaire recoupe le foyer fo.02 du Néolithique ancien.

Figure 20: East sector of Bronze age fortified settlement: detail plan of access $n^{\circ} 4$ between $2 a$ palisade and $2 d$ buildings. Post hole 369 of the intermediate gateway cuts the early Neolithic hearth fo. 02. 
comblement des tronçons du fossé $2 \mathrm{~b}$ montre un affaissement progressif du rempart au droit du bord interne du fossé (fig. 13). Entre chaque porte, la régularité des deux alignements de poteaux est régulièrement rompue par des intervalles plus larges qui semblent séparer de petits édifices. Ainsi, à hauteur de chaque élément du fossé, il est possible de distinguer trois éléments distincts. La présence de petits poteaux latéraux peut être interprétée comme un ancrage de parois en complément des deux séries de poteaux porteurs. Enfin, la dispersion du mobilier dans ce secteur (fig. 21), très pauvre certes, montre une répartition dans le grand fossé d'une part (près des entrées notamment) et sur la concentration des trous de poteau de l'autre, de part et d'autre d'une aire quasiment vierge de vestiges.

L'hypothèse de petits édifices disposés régulièrement en arc de cercle en arrière du rempart ou adossés à celui-ci semble à privilégier. À partir des éléments les mieux conservés, leur largeur peut être estimée entre 4,50 et $5 \mathrm{~m}$. D'ouest en est, et à titre d'hypothèse en fonction de l'état de conservation, la longueur d'une douzaine de ces bâtiments peut se décliner de la façon suivante (fig. 12 et 15 à 20) :

- premier tronçon du fossé : $1(10 \times 4,50 \mathrm{~m}), 2$ ( $8 \mathrm{x}$ 4,50 m), 3 (2,50 x 3,50 m-grenier?);

- deuxième tronçon : 4 ( 9 x 4,50 m), 5 ( 9 x 4,50 m), 6 $(2,50 \times 3,50$ - grenier?);

- troisième tronçon : $7(8 \times 4,50 \mathrm{~m}), 8(7,50 \times 4,50 \mathrm{~m})$, $9(6,50 \times 4,50 \mathrm{~m})$;

- quatrième tronçon : $10(9 \times 4,50 \mathrm{~m}), 11(9 \times 4,50 \mathrm{~m})$, 12 (? x 4,50 m).

La présence d'ouvertures - ou de couloirs - en partie médiane est peut-être signalée par des poteaux très rapprochés (fig. 17), par exemple TP.120-121 pour le bâtiment 1 , TP.134-135 pour le bâtiment 2, TP.170-171 pour le bâtiment 4, ou d'aménagements externes du côté sud (TP.205, 206, 208, 209) pour le bâtiment 4. Ainsi, un ensemble d'une douzaine de bâtiments à une seule nef et répartis par groupes de trois au droit de chaque tronçon du fossé peut être envisagée.

À l'extrémité occidentale de cet ensemble, un sondage a été réalisé en prolongement de l'entrée ouest et étendu sur la pente naturelle du promontoire. Hormis un léger talus de bordure dont l'aspect très terreux semble montrer une origine récente, aucune structure évidente ne semble avoir affecté la rupture de pente boisée. Cependant, plusieurs tessons de poteries ont été recueillis sous l'humus forestier et semblent signaler une zone de rejet sur la pente.

La disposition des fondations met en évidence la forte cohérence des structures entre elles et l'absence de remaniements conséquents, ce qui témoignerait d'une occupation importante pendant une durée relativement brève. L'emprise enclose par ce système défensif atteint une surface d'envi- ron 1,7 ha entre la première palissade nord et la pointe $\mathrm{du}$ promontoire (cf. infra, fig. 63). Compte tenu du volume important du fossé de barrage et probablement du rempart contigu, cet ouvrage a dû demeurer un marqueur du paysage bien après son abandon à l'âge du Bronze, même avec un profil naturellement adouci et cet élément est à prendre en considération dans l'analyse des occupations postérieures.

\section{E. Le mobilier archéologique du secteur nord (J.-Y. T.)}

Les trois datations $14 \mathrm{C}$ obtenues, l'une du fond du fossé, deux de la deuxième entrée occidentale, incendiée (voir supra, $\$$ VII B et D1) attribuent cet ensemble à la seconde moitié du Bronze moyen et au Bronze final. Notons toutefois un intervalle non négligeable entre la datation issue du fossé et celles de la porte incendiée. Faut-il y voir une installation des infrastructures internes bien après le terrassement du barrage?

Le matériel archéologique mis au jour dans ce secteur nord du site (fig. 21 à 24) est issu de trois contextes complémentaires : le nettoyage systématique du substrat, la fouille et le tamisage du contenu des trous de poteau, les sondages dans le fossé segmenté (pour lequel seules les unités stratigraphiques de base - US.3 à US.10 - sont ici prises en compte en raison du comblement récent correspondant aux US. superficielles). L'essentiel du rare mobilier de l'ensemble provient du secteur des bâtiments et, dans une moindre mesure, du fond du fossé. Sur le plan de répartition (fig. 21), une concentration particulière dans les sondages bordant l'interruption entre $2 \mathrm{~b} .1$ et $2 \mathrm{~b} .2$ indique des rejets de part et d'autre d'un accès; en revanche, la quasi-absence de matériel dans la zone intermédiaire $2 \mathrm{c}$ conforte l'hypothèse de la présence du rempart.

\section{E 1. La céramique}

Elle est représentée dans ce secteur par un total de 195 tessons pesant $2430 \mathrm{~g}$. L'importance du morcellement $-65 \%$ des tessons ont une surface inférieure à $4 \mathrm{~cm}^{2}$ - et du taux d'usure $-78 \%$ sont très érodés - indiquent un matériel fortement déplacé, caractéristique d'un site d'habitat, dans un milieu naturel agressif par son acidité. On notera que certains éléments sont mieux conservés suite à un piégeage précoce en fond de fossé ou dans les rares trous de poteau scellés par le rempart (TP.242 et 246).

Malgré cette mauvaise conservation, la série présente des caractères homogènes dans la nature des pâtes. Les teintes sont majoritairement claires (69\%), allant du beige au brun orangé. Le dégraissant est, à près de $60 \%$, composé de grains de quartz roulés et concentrés, donnant un aspect rugueux et sableux au toucher, avec $23 \%$ de gros quartz anguleux 


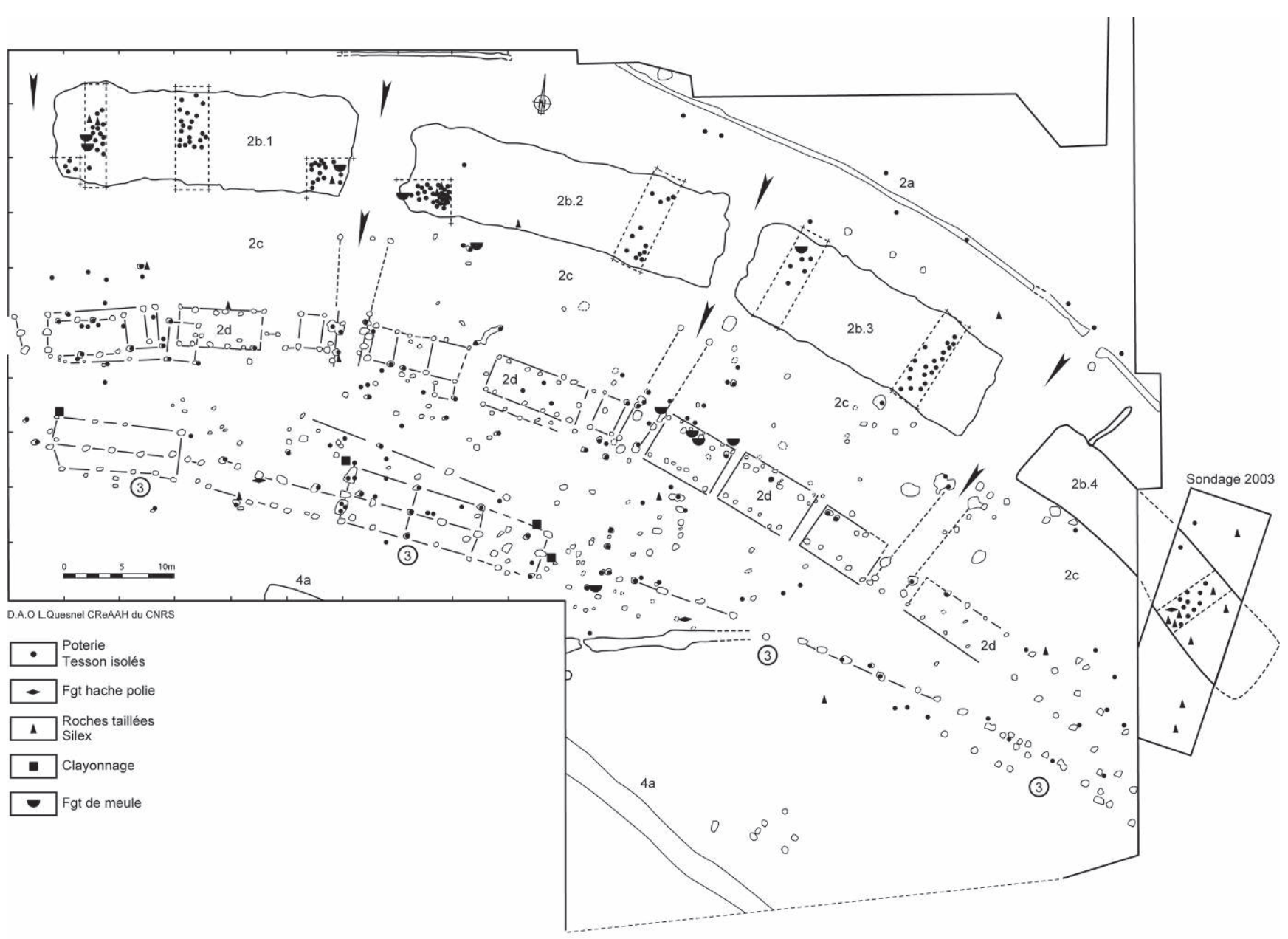

Figure 21 : Répartition du mobilier dans la zone nord sur les ensembles 2 (âge du Bronze) et 3 (premier âge du Fer).

Figure 21: Finds distribution in the north sector for wholes 2 (Bronze Age) and 3 (first Iron Age).

fréquemment visibles en surface et $17 \%$ d'éléments fins peu visibles dans une pâte homogène. Un lissage de surface n'est décelable que sur $27 \%$ des tessons mais l'agressivité du milieu est à prendre en compte. $65 \%$ des fragments ont une épaisseur de paroi inférieure à $8 \mathrm{~mm}$. Les vases de petite taille semblent majoritaires par rapport aux grands récipients à paroi épaisse.

En l'absence de formes restituables, quelques éléments particuliers nous renseignent sur les caractéristiques morphologiques de la céramique.

Les fragments de lèvres sont au nombre de 17 , dont 10 proviennent de petits vases et 7 de grands récipients à paroi épaisse. La majorité (10 cas) est à profil arrondi simple (fig. 22, no 4 à 9, 11, 12,13,15), avec épaississement de la lèvre pour cinq cas; quatre éléments sont à lèvre aplatie, dont un avec décor digité $\left(\mathrm{n}^{\circ} 16\right)$ et trois à méplat éversé signalant des récipients très ouverts $\left(\mathrm{n}^{\circ} 3,10,14\right)$. Sur les quatre fragments de carène recensés (fig. $23, \mathrm{n}^{\circ} 4,5,6,8$ ), l'un est orné d'une cupule probablement réalisé au doigt. Les six fragments de fonds plats sont épais (fig. 23, n ${ }^{\circ} 10$ à 15), sans débordement du pied, à paroi verticale (deux éléments) ou nettement oblique vers l'extérieur (trois autres).

Hormis le décor campaniforme trouvé en position secondaire dans ce secteur nord (voir supra, $₫$ VI), le décor n'est présent que sur cinq petits éléments : une pression au doigt sur lèvre (fig. $22, \mathrm{n}^{\circ} 16$ ), une légère cupule sur carène (fig. $\left.23, n^{\circ} 8\right)$, deux tessons incisés $\left(n^{\circ} 1\right.$ et 2 ), une impression à l'ongle $\left(n^{\circ} 3\right)$.

\section{E 2. Le mobilier lithique}

Il est particulièrement pauvre dans le secteur nord (fig. 24). Le silex n'y est représenté que par une vingtaine d'éclats, dont deux corticaux débités sur galets côtiers, et par un nucléus à plans de frappe bipolaires. La série est essentiellement composée de petits éclats fins; les bulbes marqués et 
Figure 22 : Mobilier céramique de la zone nord : 1, Néolithique; 2, Campaniforme ; 3-16, âge du Bronze. Figure 22: North sector ceramics: 1, Neolithic; 2, Beaker; 3-16, Bronze Age.

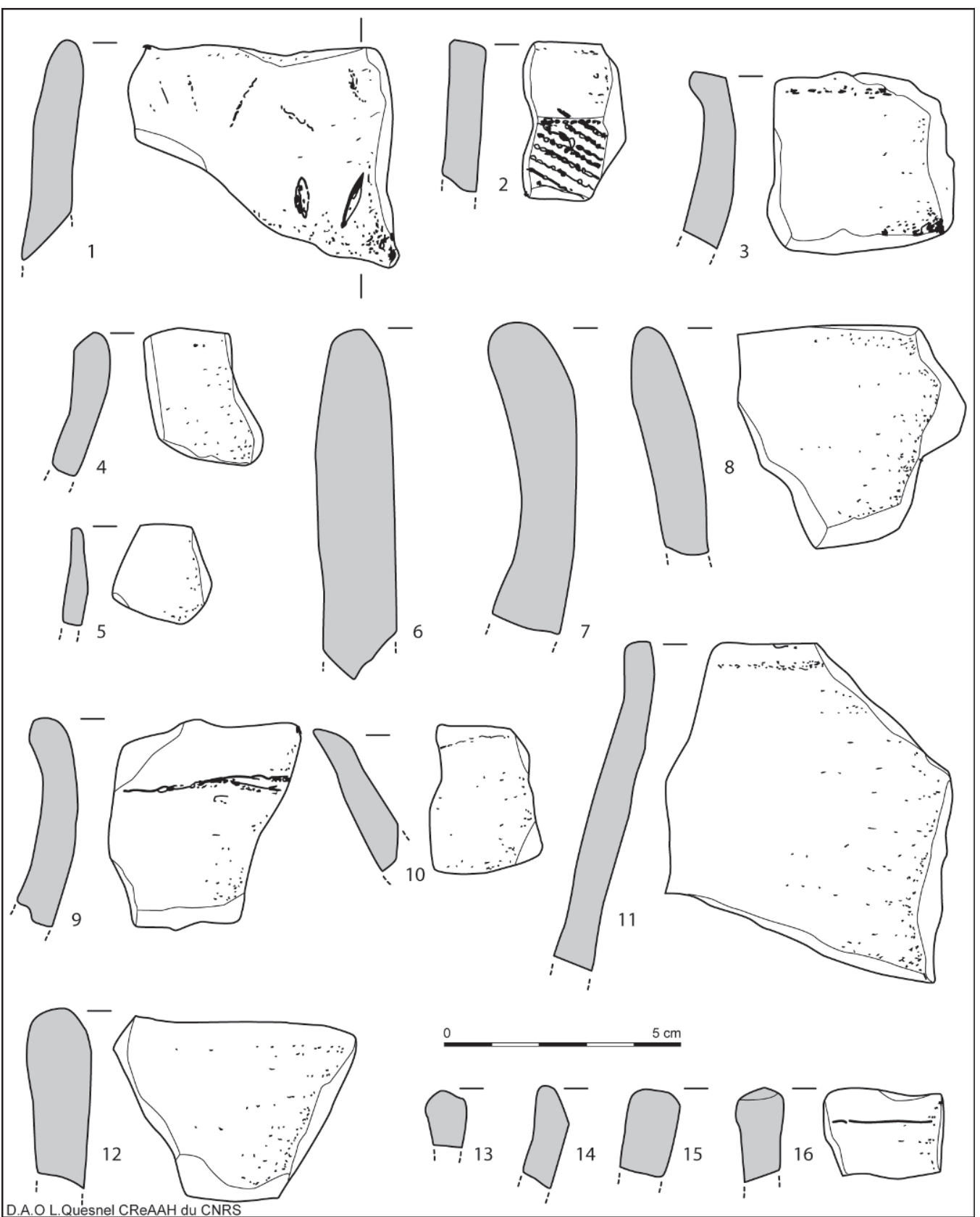

les talons lisses découlent d'un débitage direct au percuteur dur. Deux des éclats semblent sommairement aménagés en perçoir (fig. 24, $\mathrm{n}^{\circ} 9$ et 13 ) et trois éclats sont marqués de fines retouches latérales $\left(n^{\circ} 2,3,4\right)$. Le grès lustré est représenté par un nucléus à lamelles à plans de frappe croisés ( $\left.\mathrm{n}^{\circ} 14\right)$, provenant du sondage 4 du fossé $2 \mathrm{~b} .1$, et par un éclat court et large du fossé $2 \mathrm{~b} .4\left(\mathrm{n}^{\circ} 1\right)$. Ce tronçon a également livré un petit éclat d'opale résinite $\left(\mathrm{n}^{\circ} 8\right)$. Notons la présence de deux pièces en dolérite vert clair. L'un de ces blocs $\left(\mathrm{n}^{\circ} 17\right)$, venant du fond de fossé $2 \mathrm{~b} .4$, très usé, a la forme et les caractéristiques d'une ébauche de lame de hache : négatifs d'enlèvements aux extrémités, écrasement des arêtes latérales, plages bouchardées. Le second, plus massif et mis au jour au nord-ouest du site, possède plusieurs facettes de bouchardage fin et une face plane polie indiquant sa probable utilisation comme molette $\left(\mathrm{n}^{\circ} 18\right)$. La série est complétée de deux fragments de lames de haches polies en dolérite, un mésial dans le TP.22 ( $\left.\mathrm{n}^{\circ} 15\right)$ et un tranchant $\left(n^{\circ} 16\right)$ sur le substrat, dans le secteur de la palissade de l'ensemble 3 du premier âge du Fer. La phase de décapage a fourni une lame retouchée en quartzite fin et gris clair (quartzite de Montbert?) ( $\left.\mathrm{n}^{\circ} 11\right)$. 


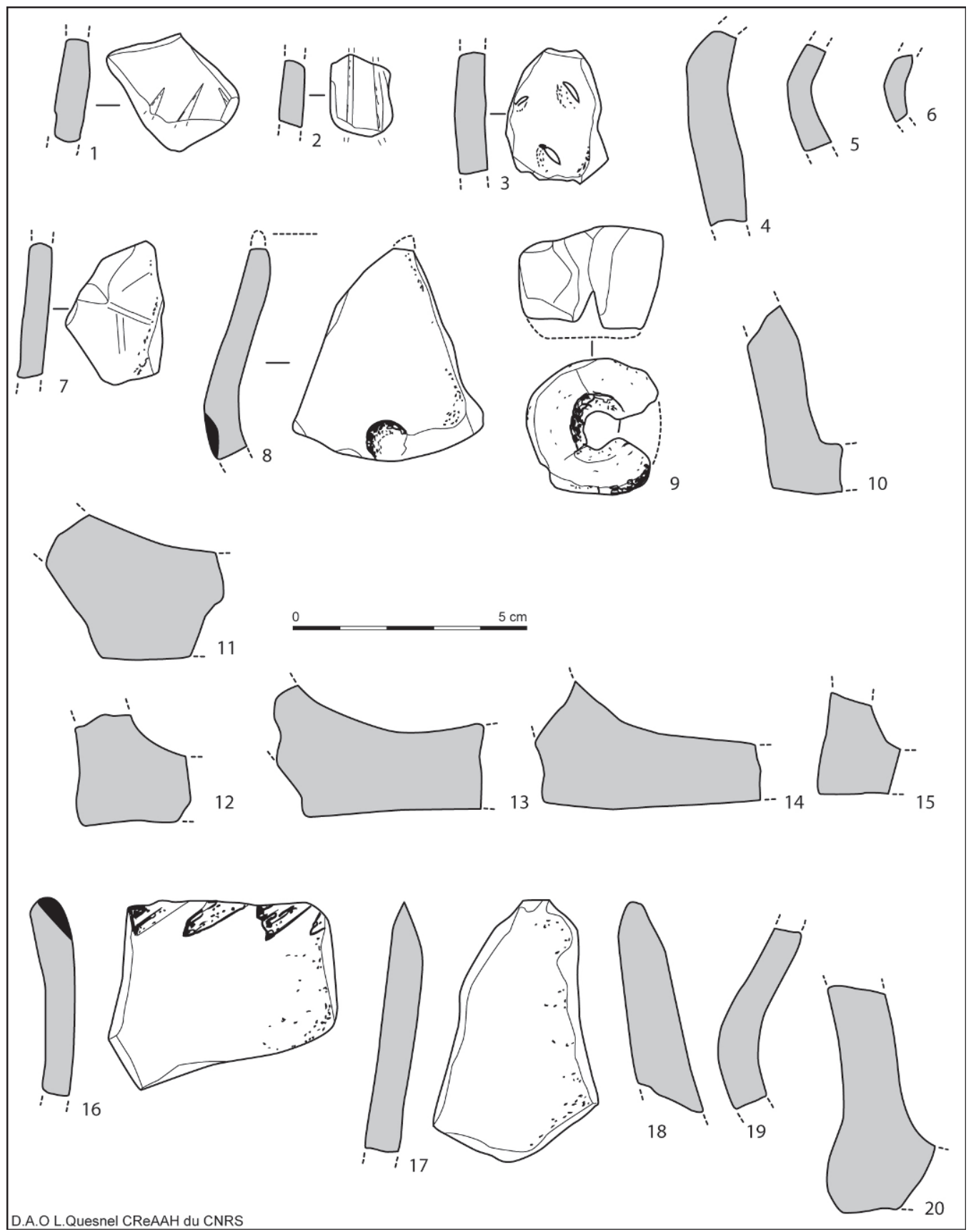

Figure 23 : mobilier céramique de la zone nord : 1-15, âge du Bronze; 16-20, âge du Fer.

Figure 23: North sector ceramics: 1-15, Bronze Age; 16-20, Iron Age.

\section{E 3. Le macro-outillage (K. D.)}

L'éperon barré de La Rochette n'a livré que peu de macro-outillage, à l'instar des autres catégories de mobilier. 45 pièces en roches exogènes ont été collectées, pour un poids total de plus de $34,5 \mathrm{~kg}$. Seules onze d'entre elles portent des traces d'utilisation et peuvent par conséquent être considérés comme des outils.

a) Les matières premières. Elles seront étudiées dans un premier temps, indépendamment des éventuels outils que

portent ces supports. Tous les fragments de roches exogènes sans trace d'utilisation seront également pris en compte puisqu'ils ont été apportés par l'homme sur le site et qu'ils ont pu faire partie d'outils détruits.

Les catégories pétrographiques ont été définies à l'œil nu. La composition du corpus est largement dominée par le poudingue, le granite et le grès fin (tabl. 2). Les autres roches identifiées sont la dolérite, le gneiss, le quartz et le quartzite. Deux types de granite se distinguent : une roche claire légèrement pegmatitique et un granite sombre avec 


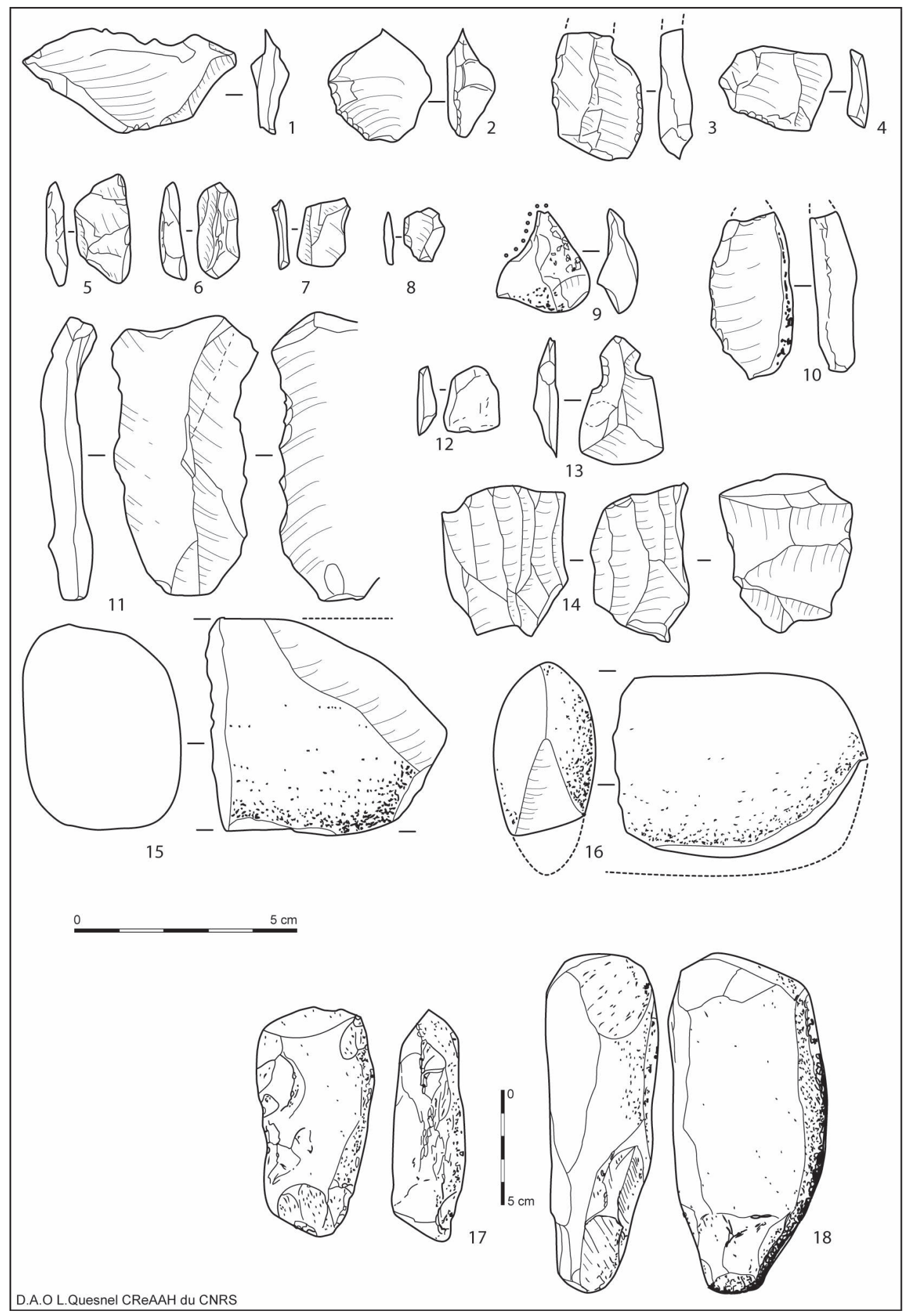

Figure 24 : Mobilier lithique de la zone nord : 1 et 14 , grès lustré; $2-7,9,10,12,13$, silex; 8 , opale- résinite; 11, quartzite (de Monbert?) ; 15-18, dolérite.

Figure 24: North sector lithics: 1 \& 14, «lustred » sandstone; 2-7, 9, 10, 12, 13, fint; 8, "opale-resinite 》 (opaline); 11, (Montbert?) quartzite; 15-18, dolerite. 


\begin{tabular}{|c|c|c|c|c|c|}
\hline \multirow{2}{*}{$\begin{array}{c}\text { Natures } \\
\text { pétrographiques }\end{array}$} & \multicolumn{3}{|c|}{ Nombre de pièces } & \multicolumn{2}{c|}{ Poids des matériaux } \\
\cline { 2 - 6 } & Supports & Total & $\%$ & 857 & 2,5 \\
\hline Dolérite & 1 & 1 & 2,2 & 1285 & 3,7 \\
\hline Gneiss & 1 & 1 & 11,1 & 11012 & 31,8 \\
\hline Granite clair & - & 5 & 2,2 & 4580 & 13,3 \\
\hline Granite à tourmaline & 1 & 1 & 33,4 & 4895 & 10014 \\
\hline Grès fin & - & 15 & 40 & 1670 & 29 \\
\hline Poudingue & 4 & 18 & 6,7 & 248 & 4,8 \\
\hline Quartz & 3 & 3 & 2,2 & 34561 & 0,7 \\
\hline Quartzite & 1 & 1 & 100 & & 100 \\
\hline TOTAL & 11 & 45 & & & \\
\hline
\end{tabular}

Tableau 2 : Nature pétrographique du macro-outillage.

Table 2: Macro-tools petrography.

énormément de tourmaline. Ces matières premières sont présentes en proportions très variables. De nombreuses roches sont absentes sous forme brute (dolérite, gneiss, granite à tourmaline, quartz et quartzite), tandis que le grès fin et le granite clair n’ont servi à la confection d'aucun outil reconnu, bien qu'ils aient été apportés sur le site.

La forte fragmentation du corpus et l'existence de nombreux fragments sans trace d'utilisation rendent toute relative l'évaluation du nombre des pièces. La pesée des matériaux, considérant le volume de matière première stricto sensu et indépendamment du nombre de support fournis, permet une meilleure évaluation des apports anthropiques sur le site (tabl. 2). Le granite clair est ainsi la plus importante roche exogène du corpus, suivi du poudingue. Pour l'analyse de l'outillage, un décompte des supports utilisés est plus pertinent, faisant apparaître la prépondérance du poudingue, suivi du quartz.

Quel que soit leur matériau, les supports de macro-outils sont de différents types. On distingue les blocs, les galets et les dalles et plaquettes; à ces supports naturels s'ajoute une hache polie recyclée. Le corpus est très largement dominé par les blocs, avec 38 individus sur 45. Les galets constituent le deuxième type de support privilégié (5 pièces) et l'utilisation d'une plaquette et d'une hache polie peut être considérée comme anecdotique. Ces types de supports sont issus de milieux différents.

Les galets montrent le plus souvent un poli fluviatile; l'origine de ces supports est donc probablement locale, collectés dans les cours d'eau du voisinage du site; ils peuvent également provenir des alluvions de ces mêmes cours d'eau. Les roches concernées par ce mode d'approvisionnement sont surtout le quartzite (un unique support) et le quartz (deux supports sur trois).

La plupart des blocs portent des altérations atmosphériques ou hydrauliques montrant qu'ils ont été collectés déjà détachés du substrat et qu'ils n’ont pas nécessité une extraction. L'intégralité des supports en granite clair et en grès fin sont des blocs ainsi que presque tout ceux en poudingue. Ce sont les matières les plus représentées sur le site.

C'est donc en milieu terrestre que se faisait l'essentiel de la collecte des roches nécessaires au macro-outillage. Le grès fin abonde dans le secteur, par niveaux coiffant les reliefs à $1 \mathrm{~km}$ au nord du site et à $0,5 \mathrm{~km}$ au sud-est de celui-ci (Thomas et Carn, 2008). Le poudingue se trouve en bancs au sommet des reliefs, sur les niveaux gréseux. Au plus proche du site, cette roche se trouve à $4 \mathrm{~km}$ au sud (Thomas et al., 2004). C'est le granite qui a l'origine la plus lointaine, le massif le plus proche du site étant celui de Plémet-Goméné, à $11 \mathrm{~km}$ au nord-ouest (Thomas et Carn, 2008). L'unique plaquette de gneiss peut provenir du même massif. Quant au support en dolérite, il s'agit d'une ancienne hache polie, probablement récupérée sur le site même ou à proximité immédiate. Cette diversité en roches exogènes, malgré les nombreux blocs restés bruts, témoigne d'une exploitation optimale des ressources lithiques environnantes.

b) L'outillage. Considérons maintenant les seuls éléments portant des traces d'utilisation qui, au nombre de onze, constituent le macro-outillage à proprement parler. Chaque trace d'utilisation correspond à un mode de fonctionnement (d'après la typologie des percussions d'A. Leroi-Gourhan, 1971, p. 47-60) et renvoie à une fonction, considérée ici à titre d'hypothèse. Un des supports s'est vu attribuer deux hypothèses fonctionnelles différentes car il porte deux types de traces et correspond donc à deux outils distincts (Donnart, 2010). Il porte le nombre total d'outils à douze. Seules quatre hypothèses fonctionnelles différentes ont été déterminées (tabl. 3), ce qui est peu pour un habitat d'une telle ampleur où l'on peut s'attendre à une plus grande diversité des fonctions. 


\begin{tabular}{|l|c|c|c|c|c|c|c|}
\hline \multicolumn{1}{|c|}{$\begin{array}{c}\text { Nature pétrographique } \\
\text { Hypothèses fonctionnelle }\end{array}$} & Dol & Gn & GT & Pou & Qz & Total \\
\hline Boucharde & - & - & - & - & 3 & - & 3 \\
\hline Meule & - & - & 1 & 4 & - & - \\
\hline Molette & 1 & - & - & - & - & - & 2 \\
\hline Percuteur & - & - & - & - & - & 1 & 1 \\
\hline TOTAL & 1 & 2 & 1 & 4 & 3 & 1 & 12 \\
\hline
\end{tabular}

Tableau 3 : Décompte des hypothèses fonctionnelles pour le macro-outillage en fonction des natures pétrographiques (Dol : dolérite; Gn : gneiss; GT : granite à tourmaline; Pou : poudingue; Qz : quartz; Que : quartzite).

Table 3: Macro-tools functional hypotheses in relation with petrography.

Ce corpus est très fragmenté : seuls quatre des onze supports sont entiers (taux de fracturation $64 \%$ ). Le taux de pièces brûlées est également élevé : avec quatre pièces, il atteint $36 \%$. Ce mauvais état de conservation signe une longue présence humaine sur le site. Le très faible taux de supports multifonctionnels est par contre en contradiction avec ce constat, puisque les occupations de longue durée produisent habituellement de nombreux outils multifonctionnels (Donnart, 2007). La forte fragmentation du corpus peut cependant entraîner une sous-évaluation de ce taux.

L'assemblage se caractérise par plusieurs modes de fonctionnement, dont les deux grandes classes sont la percussion lancée et la percussion posée (Leroi-Gourhan, 1971, p. 47-60). Le premier mode concerne les bouchardes et le percuteur; le second est représenté par l'outillage de mouture. La percussion posée est ici dominante, avec huit outils. Les quatre hypothèses fonctionnelles sont présentes en proportions variables (tabl. 3), avec une dominance des instruments de mouture, suivis des bouchardes.

L'outillage de mouture consiste en moulins va-et-vient, composés d'une meule dormante et d'une molette. Six fragments ou groupes de fragments de meules ont été identifiés, ainsi que deux molettes. Seule une d'entre-elle est entière, montrant la très grande fracturation de cet outillage (taux de $87 \%$ ). Les supports des meules sont tous des blocs, sauf un galet fluviatile. Quatre sont en poudingue, un en gneiss et le galet est en granite à tourmaline. Leur morphologie initiale est très difficilement restituable vu leur fragmentation mais l'exemplaire sur galet avait probablement une forme ovalaire (fig. $25, n^{\circ} 1$ ). Des traces de façonnage du support sont visibles sur un fragment en poudingue, dont l'angle a été arrondi par piquetage $\left(n^{\circ} 2\right)$. Les parties actives en sont toutes légèrement concaves. Leur contour semble être ovale et s'arrête le plus souvent avant d'atteindre le bord du support. Deux fragments présentent clairement une réduction de leur surface active (fig. $25, n^{\circ} 2$ et fig. 26, n²) : celle-ci était quasi plane dans un premier temps puis, lors d'un ravivage, la surface a été réduite (ou recentrée?). La poursuite de son utilisation a creusé la surface ainsi redéfinie, entraînant la formation d'une arête au bord de la surface active. De telles réductions de la surface active ont été observées sur des meules, plus anciennes, du site campaniforme de Begar-Loued (Molène, Finistère), mais elles intervenaient à des stades beaucoup plus avancés d'usure (Donnart, à paraitre). Les parties actives sont toutes préparées par un piquetage régulier, qui tend à disparaître sous le poli d'utilisation. Ce poli ne se produit pas de façon uniforme et semble affecter en premier lieu les bords de la surface active (fig. $25, \mathrm{n}^{\circ} 1$ ).

Contrairement aux meules qui présentent une certaine homogénéité morphologique, les deux molettes sont très différentes l'une de l'autre. La première a pour support une plaquette de gneiss, issue d'une meule fragmentée (fig 25, $\left.\mathrm{n}^{\circ} 3\right)$. Sa partie active couvre toute la surface du support; elle est légèrement convexe et a été préparée par piquetage. Les traces d'utilisation, sous forme de poli, recouvrent les sommets du micro relief et affectent en priorité les bords de l'outil. Ces caractéristiques (courbure et préparation de la surface active, modalités d'apparition des traces d'utilisation) concordent avec une utilisation allant de pair avec les meules du site. L'autre molette, seul outil de mouture entier, differe tant par ses traces d'utilisation que par son support (fig. 26, $\mathrm{n}^{\circ} 3$ ). Ce dernier est une ébauche de hache polie : le bloc prismatique de dolérite présente quelques négatifs d'enlèvements partiellement oblitérés par un bouchardage extensif. Ce support opportuniste a été mis à profit sans plus de préparation pour en faire une molette sur sa face la plus plane. Les traces d'utilisation sont un poli accompagné de nombreuses stries subparallèle, transversales au support, témoignant d'une utilisation en un mouvement de va-et-vient. La surface active est plane voire très légèrement concave, et pourtant le poli y est uniformément réparti; cette molette n'a donc pas pu servir sur une meule classique, à la surface active concave. Sa fonction diffère très probablement de celle des autres outils de mouture étudiés; elle a pu servir à broyer un matériau dur (d'où les nombreuses stries) sur une dalle à moudre (simple support lithique plat). 


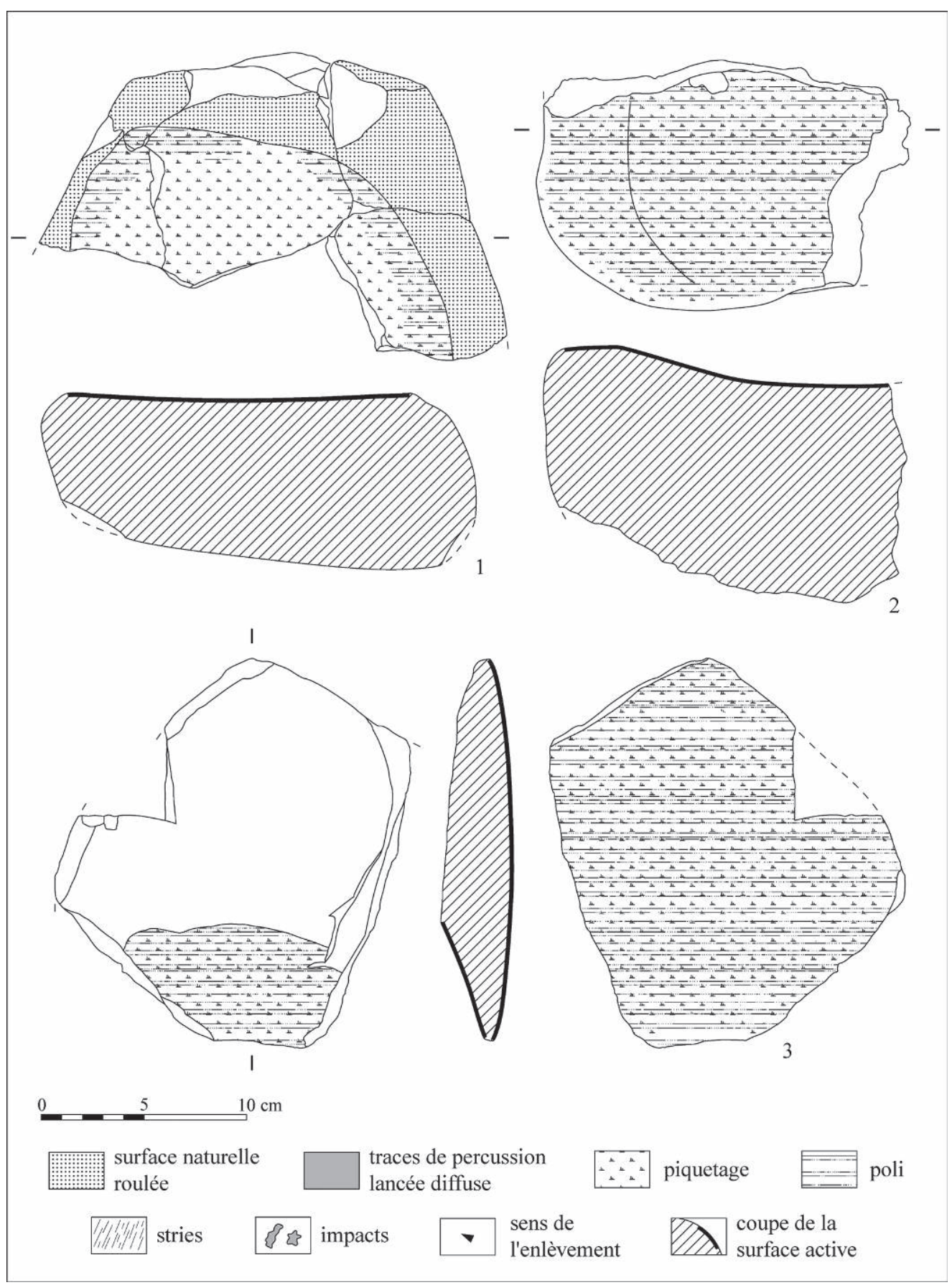

Figure 25 : Instruments de mouture : 1 , meule sur galet, granite à tourmaline; 2 , meule, poudingue; 3 , fragment de meule réutilisé en molette, gneiss (DAO Klet Donnart).

Figure 25: Grinding instruments: 1, Quern on granite pebble; 2, Quern, puddingstone; 3, fragment of quern re-used as a rubber, gneiss.

Les bouchardes sont des outils fonctionnant en percussion lancée diffuse; elles different des percuteurs ordinaires du fait qu'il n'y a pas dans leur utilisation de recherche d'un point de percussion précis (Le Roux, 1999, p. 101). La percussion se fait dans le but de travailler une surface, ce qui forme des traces beaucoup plus couvrantes (Sestier et Bontemps, 2003). Les trois bouchardes identifiées à La Rochette sont toutes en quartz. Deux sont sur galets et une sur bloc; une seule est brisée (fig. $26, \mathrm{n}^{\circ}$ 4). Les traces d'uti- lisation sont des écrasements de la roche, consécutifs à une forte percussion lancée et parfois accompagnés de négatifs d'enlèvements accidentels. Les écrasements sont disposés en plages planes ou convexes, qui peuvent être très étendues $\left(\mathrm{n}^{\circ} 4\right)$ et même modifier la morphologie originelle des supports $\left(\mathrm{n}^{\circ} 5\right)$. Ces stigmates sont typiques du travail de la pierre (Poissonnier, 2002; Sestier et Bontemps, 2003). Les bouchardes ont probablement servi à l'entretien des instruments de mouture du site : les surfaces actives des meules et 

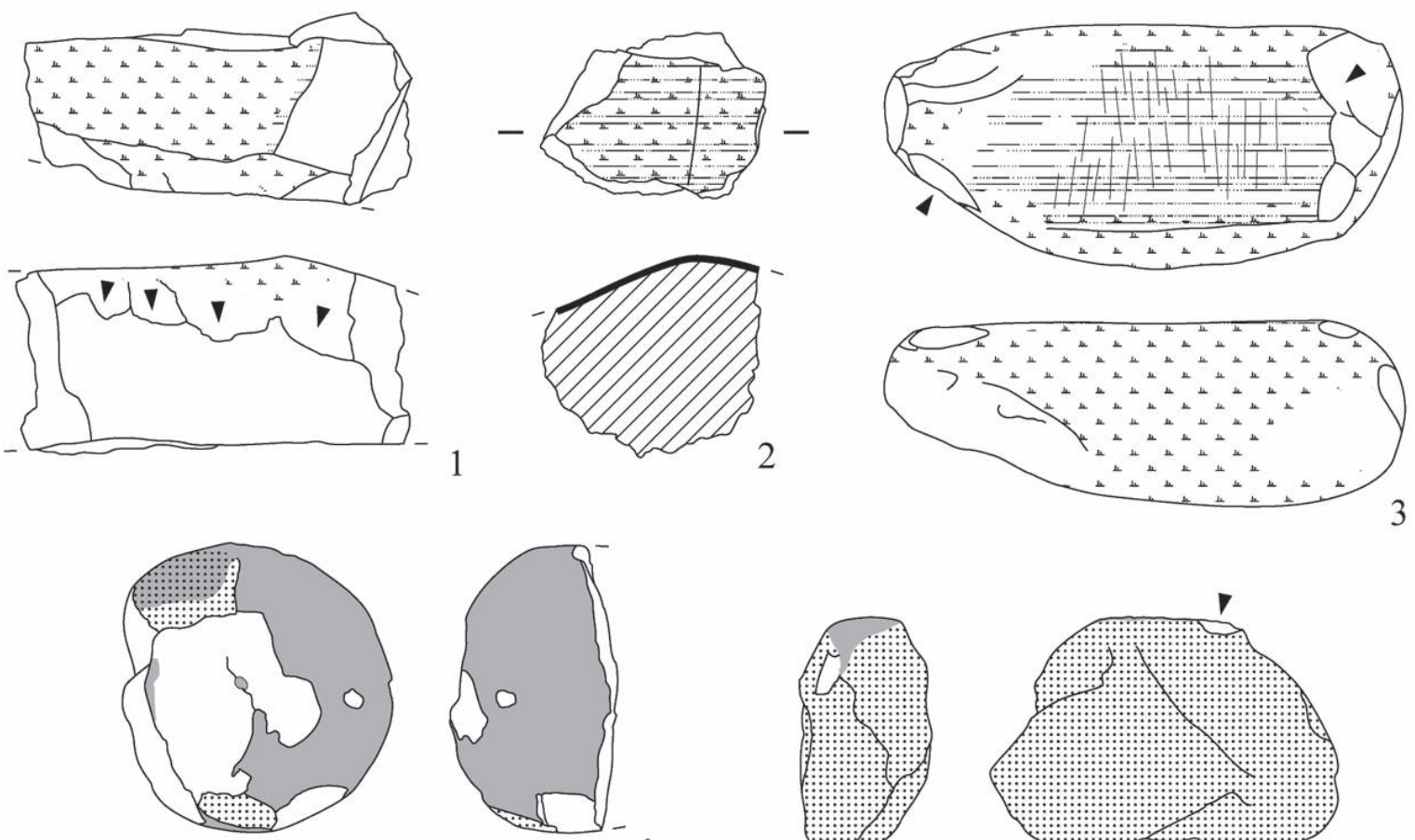

4
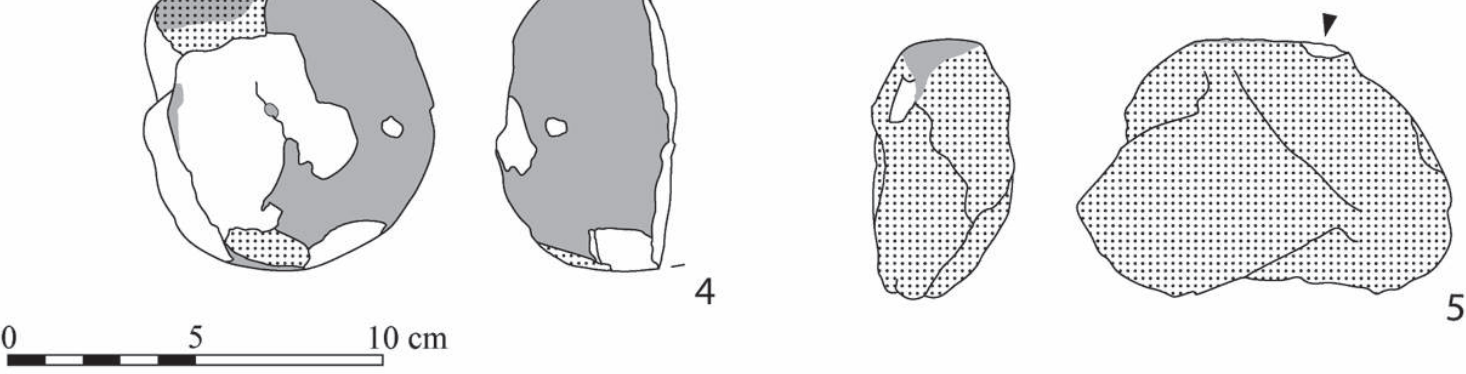

Figure 26 : Macro-outils divers : 1 et 2, fragments de meules, poudingue; 3, molette sur ébauche de hache, dolérite; 4 et 5, bouchardes, quartz (DAO Klet Donnart).

Figure 26: Various macro-tools: $1 \& 2$, Quern fragments, puddingstone; 3, rubber on a stone axe rough-out, dolerite; 4 \& 5, hammers, quartz.

des molettes doivent être régulièrement piquetées pour leur redonner du mordant.

L'unique percuteur du site, dont le support entier est un galet de quartzite, a servi sur ses deux extrémités pour quelques percussions lancées contre une matière dure.

c) Apports à l'interprétation du site. La confrontation de cette analyse technologique avec les données de terrain peut apporter des éléments de compréhension pour les différentes occupations du site. La plupart des pièces proviennent de trous de poteaux et y étaient vraisemblablement placées comme éléments de calage. Le grand fossé interrompu $2 \mathrm{~b}$ a livré un certain nombre de blocs de roches exogènes, mais un seul d'entre eux a été employé (fig. 26, n 5). Il apparaît que la zone nord du site est la plus riche en macro-outils, mais sans atteindre une densité suffisante pour parler d'aire d'activité spécialisée. Leur dispersion les rattache plutôt aux activités domestiques liées à l'habitat.

La datation précise des ensembles architecturaux fournit des terminus ante quem aux outils usagés réutilisés en calages de poteaux mais ne date pas leur utilisation. Plus de la moitié des pièces provenant des structures en creux du Bronze moyen-final (six sur onze), elles ont pu être utilisées au début de cette même phase, mais également être récupérées sur le site ou ailleurs. Le placement de ces outils en guise de calage dans les trous de poteau pourrait montrer qu'une présence humaine de type habitat était déjà en place sur le site au moment de l'édification des structures Bronze moyen-final. Les occupations ultérieures n'ont livrées chacune qu'une seule de ces pièces. Les fragments de la meule sur galet (fig. $25, \mathrm{n}^{\circ} 1$ ) servaient de calage dans un trou de poteau du premier Âge du Fer. Un autre fragment de meule (fig. 26, $\mathrm{n}^{\circ} 2$ ) a été trouvé près du fossé du second âge du Fer. La similitude entre les meules du Bronze moyen-final et celles de l'âge du Fer s'explique par la perduration de ces instruments due à l'apparition tardive et progressive du moulin rotatif à l'âge du Fer (Pommepuy, 1999). Enfin, l'unique percuteur provient d'un trou de poteau du Moyen Âge.

Chacun des macro-outils renvoie à une activité précise qui s'est déroulée sur place. Le spectre fonctionnel de l'assem- 
blage est relativement limité et dominé par les instruments de mouture (tabl. 3). La fonction de boucharde se rapporte à une percussion lancée diffuse sur de la pierre et ces outils pourraient très bien être ceux qui ont servi au façonnage et à l'entretien des meules et molettes. Cet assemblage quasi exclusivement orienté vers la mouture traduit une activité typiquement domestique caractéristique du fonctionnement d'un habitat.

\section{E 4. L'attribution culturelle du mobilier du secteur nord}

La série lithique, peu caractéristique, marque probablement la présence d'un fond néolithique résiduel, mais sans possibilité de la rattacher à une période précise. Piégés dans les fondations des occupations postérieures, voire réutilisés (comme les blocs de dolérite par exemple), ces éléments ont semé le doute sur la datation de cet ensemble fortifié lors des premières campagnes de fouille.

Bien qu 'également très indigente, la série céramique présente toutefois quelques caractères plus en adéquation avec les datations obtenues par le radiocarbone. La qualité des pâtes de certaines pièces fines, les quelques décors au doigt, à l'ongle et incisés, les lèvres à méplat de récipients à large ouverture sont autant d'éléments contribuant à une datation de cet ensemble nord à l'âge du Bronze moyen et final. Quelques rapprochements peuvent être évoqués avec l'importante série mise au jour à Mez-Notariou sur l'île d'Ouessant (Le Bihan [dir], 2010), tels que le décor digité sur la lèvre (cf. p. 410, pl. 15, n 158-05) et le décor incisé oblique (p. 464, pl. 69, n 59-1031 et p. 465, pl. 70, n 59-1031). De même, la petite pièce de céramique perforée et fragmentée provenant du fossé $2 \mathrm{~b} 2$ (fig. $22, \mathrm{n}^{\circ} 16$ ) peut être interprétée comme une fusaïole grossière, voire comme un moule de petit objet métallique à l'image de celui de Mez-Notariou (ibid., p. 426, pl. 31, n 325-04).

\section{F. Comparaison avec certains sites fortifiés de l'âge du Bronze dans l'Ouest de la France (J.-Y. T.)}

En Bretagne, le site le plus proche de La Rochette, morphologiquement et géographiquement, est celui du ValAubin à Lamballe (Côtes-d'Armor) (fig. 27, no 3), à une cinquantaine de kilomètres au nord. La prospection aérienne est également à l'origine de sa découverte en 1989. Il s'agit d'un promontoire triangulaire dominant par un fort abrupt la petite rivière Le Gast. À l'instar de La Rochette, un fossé arqué, segmenté en au moins six tronçons, ceinture une emprise d'environ un hectare. Le site a fait l'objet de deux sondages d'évaluation en 2001 (Hamon, 2001). Excavé également dans un substrat schisteux, le fossé de barrage présente un profil et une profondeur proches de ceux de La Rochette : fond plat et parois très obliques ou subverticales, un mètre de profondeur dans le rocher. Seule, sa largeur, de quatre mètres environ, est nettement inférieure. De même, la dynamique de comblement présente des similitudes avec notamment les effondrements successifs de matériaux contre la paroi interne, interprétés comme la dégradation progressive d'un talus bordier. Le rare matériel archéologique indique une occupation du Néolithique final au Moyen Âge, avec toutefois des points forts au Bronze final et à la fin de l'âge du Fer. À l'image de La Rochette, une datation sur charbon provenant de la couche d'éboulis au fond du fossé confirme l'occupation du Bronze final : Tucson : $2870 \pm$ 45 B.P., soit 1210 à 898 av. J.C. Aucune structure interne n'a été repérée mais cette absence peut être due à la l'extension limitée des sondages.

À Brandivy (Morbihan), au lieu-dit Kerlande, deux enclos accolés ont été partiellement étudiés lors d'une intervention de sauvetage en 1971 et 1972 (Lecornec, 1973). Les restes de l'enclos sud-est, de forme elliptique ( $40 \times 32 \mathrm{~m})$, montrent un rempart en moellons de granite large de 1,50 m pour une hauteur primitive estimée à $1,50 \mathrm{~m}$ au vu du volume des éboulis. Adossée au parement interne du rempart, une structure d'habitat était composée d'un muret bas périphérique doublé de poteaux, dont un exemplaire central fortement calé. Avec un mobilier céramique attribuable au Bronze final et au premier âge du Fer, plusieurs datations sur charbons furent obtenues, dont une se rapporte au Bronze final : Gif-2378: $2820 \pm 100$ BP, soit 1270-800 av. J.-C. en âge calibré.

Plus récemment, plusieurs entités similaires ont été découvertes grâce aux clichés aériens (Gautier, 1996; 2002). Au sud de la commune de Mauron, le site de La Ville-es-Zalo (fig. 27, $n^{\circ} 2$ ) ceinture un léger promontoire au dessus de la vallée du Doueff et d'une zone marécageuse. Plus à l'ouest, le site de Beg-ar-Menez-Traon à Quimper $\left(n^{\circ} 4\right)$ surplombe la vallée encaissée de l'Odet. Même si l'attribution culturelle des deux derniers reste à confirmer, ces quatre sites ont en commun une position topographique dominante particulière, un fossé de barrage segmenté et une surface enclose de un à deux hectares.

En Basse-Normandie, l'intérêt pour les sites fortifiés de hauteur à multiples occupations au cours de la Protohistoire est relancé dans le cadre d'un Projet collectif de Recherche. On retiendra notamment l'éperon du Crochemélier à Igé (Orne), barré d'un talus doublé d'un large fossé et protégeant une emprise de $8000 \mathrm{~m}^{2}$. Une opération de sondages réalisée en 2007 (Delrieu, 2007) y a confirmé une occupation homogène au Bronze final III et mis en évidence, à l'intérieur de l'enceinte, la présence de petits bâtiments (10$15 \mathrm{~m}^{2}$ ) fondés sur poteaux ou sablières basses selon la nature 


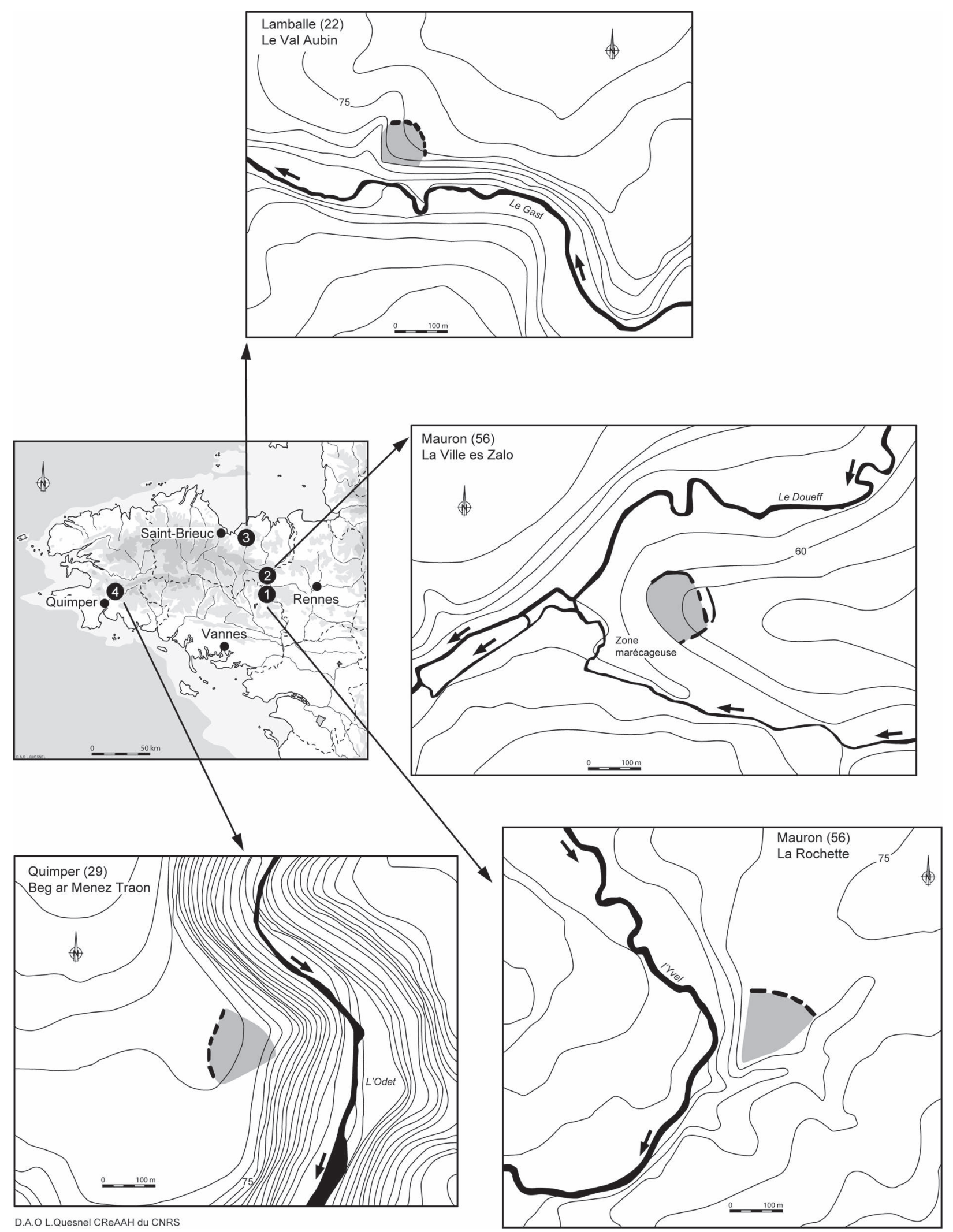

Figure 27 : Quelques sites de promontoires barrés d'un fossé segmenté en Bretagne (prospections Maurice Gautier : $\mathrm{n}^{\circ}$ 1, 2, 4; Louis Andlauer : $\mathrm{n}^{\circ} 3$ ).

Figure 27: Examples promontories fortified by a segmented ditch in Brittany. 
du substrat sous-jacent. Ce site, d'emprise relativement modeste à l'image de La Rochette, est contemporain d'ensembles plus conséquents comme le Mont Joly (Calvados) et le Camp de Bierre à Merri (Orne).

En Haute-Normandie, le suivi préventif de l'autoroute A28 dans l'Eure est à l'origine de la découverte en 2003 d'un site exceptionnel du Bronze final au Buisson du Roui à Malleville-sur-le-Bec (Mare, 2003; 2005). Étudié sur une vaste emprise de 4,5 ha, cet ensemble associe une enceinte circulaire fossoyée à plus d'une vingtaine de bâtiments circulaires et à une nécropole localisés à l'extérieur de l'enceinte selon des secteurs bien déterminés et spécialisés. Fouillée sur un peu plus de la moitié de sa surface, la morphologie de l'enceinte de Malville rappelle celle de La Rochette par certains aspects : la segmentation du fossé, l'aménagement d'une des entrées, la présence de séries de palissades internes concentriques au fossé notamment. Les vestiges de structures sur poteaux sont également visibles en zone interne.

Ce type d'enceinte circulaire à fossé segmenté trouve des parallèles dans le sud-est de l'Angleterre. On y retiendra particulièrement les enceintes de Mucking North Ring et Springfield Lyons dans le comté d'Essex (Brown, 1996; 2001). Cette dernière est déterminée par un fossé circulaire scindé en six tronçons de longueurs inégales; l'interruption qui semble correspondre à l'entrée principale est orientée à l'est à l'instar de Maleville et structurée d'un puissant porche en partie interne. Ici également, deux cercles parallèles de poteaux, concentriques au fossé, bordent un espace vierge probablement destiné au rempart interne. Ces enceintes protègent chacune quatre bâtiments circulaires et la surface enclose varie de 1600 à $3000 \mathrm{~m}^{2}$.

Dans le centre-ouest de la France, l'éperon barré du Camp Allaric à Aslonnes (Vienne) est une comparaison incontournable pour La Rochette (Pautreau et Matay, 2007). Tout d'abord, le choix d'un promontoire triangulaire dominant la confluence de la Clouère et du Clain est remarquable. La surface légèrement supérieure à 2 ha, enclose par le rempart doublé d'un fossé, est proche de celle de Mauron. Le rempart est principalement attribuable au Bronze final IIIb et le fossé à parois verticales et fond plat, profond seulement de 0,60 $\mathrm{m}$ semble avoir servi de carrière pour l'édification du talus. De petites habitations de 25 à $30 \mathrm{~m}^{2}$ étaient construites sur poteaux au pied du rempart. L'occupation du second âge du Fer est également marquée par une réduction importante de l'espace occupé à la pointe de l'éperon.

Dans le Bassin parisien, nombreux sont les éperons barrés occupés au Bronze final et d'une surface de quelques hectares à l'instar de celui de La Rochette. Certains ont fait l'objet de sondages et de fouilles.

Le Camp César à Catenoy (Oise) occupe une pointe triangulaire à l'extrémité d'un vaste plateau calcaire (Blanchet,
1984; Blanchet et Talon, 1987). Un large fossé bordé d'un rempart isole du plateau une surface de près de cinq hectares. En bordure nord du promontoire, des vestiges d'habitations rectangulaires, sur semelles de fondation en pierre ou sur trous de poteau, ont été mis au jour. Le site, dominant une vaste zone marécageuse, est localisé non loin d'une ancienne voie de communication et sa position stratégique a été évoquée.

L'éperon du Châtelet à Boulancourt (Seine-et-Marne) présente également un système fortifié complexe daté du Bronze final IIIb, protégeant un promontoire triangulaire allongé dominant la vallée de l'Essonne (Simonin, 1996; Balasescu et al., 2008); la surface enclose avoisine un hectare. La fortification est constituée d'une succession de barrages parallèles : un alignement d'une dizaine de fosses en partie externe, un fossé large d'une dizaine de mètres ayant probablement servi de carrière pour le rempart, un fossé plus étroit et régulier, une double rangée de trous de poteau interprétée comme l'armature du rempart. En partie interne, deux ensembles de poteaux dessinent des modules quadrangulaires accolés ( $3 \times 4 \mathrm{~m}$ ), l'un parallèle au rempart, le second en bordure sud du promontoire où une porte est aménagée dans la fortification, dans l'axe des bâtiments sud. La similitude morphologique avec La Rochette est indéniable, tant sur le plan de la fortification que sur celui des habitats internes. Par ailleurs, l'étude de l'abondante faune conservée à Boulancourt met en évidence la présence d'animaux d'élevage et une forte consommation carnée qui reflète probablement l'alimentation d'un milieu social privilégié (Balasescu et al., 2008). Sur le site de La Rochette également, l'entretien (avec apport de fourrage) d'un cheptel sur le vaste espace (plus d'un hectare) disponible en partie interne n'est pas à exclure, mais sans que l'on puisse l'attester en raison de la forte acidité des sols incompatible avec la préservation des restes de faune.

\section{L'occupation de L'ÂGe du Fer (J.-Y. T., J. V. D. P., C. O.)}

\section{A. Une palissade du premier âge du Fer}

À une distance vers le sud variant de trois à sept mètres de l'ensemble précédent, une cinquantaine de trous de poteau, distants l'un de l'autre de deux à trois mètres, dessinent une palissade légèrement arquée à l'instar de la structure précédente, mais marquant toutefois une nette distorsion dans le parallélisme, notamment en partie est (fig. 4, 15, 16; fig. 28). Ces fondations s'en distinguent également par un plus grand diamètre d'ouverture $(0,50$ à $0,80 \mathrm{~m})$, une profondeur moindre $(0,15$ à $0,20 \mathrm{~m})$ et surtout un com- 
Figure 28 : Secteur ouest de l'ensemble 3 : palissade du premier âge du Fer (TP.24, 26, $43-45,65,72,75)$ et structures associées; coupes des trous de poteau et datations 14C (en encadrés). Figure 28: West sector of the whole 3, first Iron Age palisade (TP.24, 26, 43-45, 65, $72,75)$ and associated structures, post-holes sections and $14 \mathrm{C}$ datings (inserts).

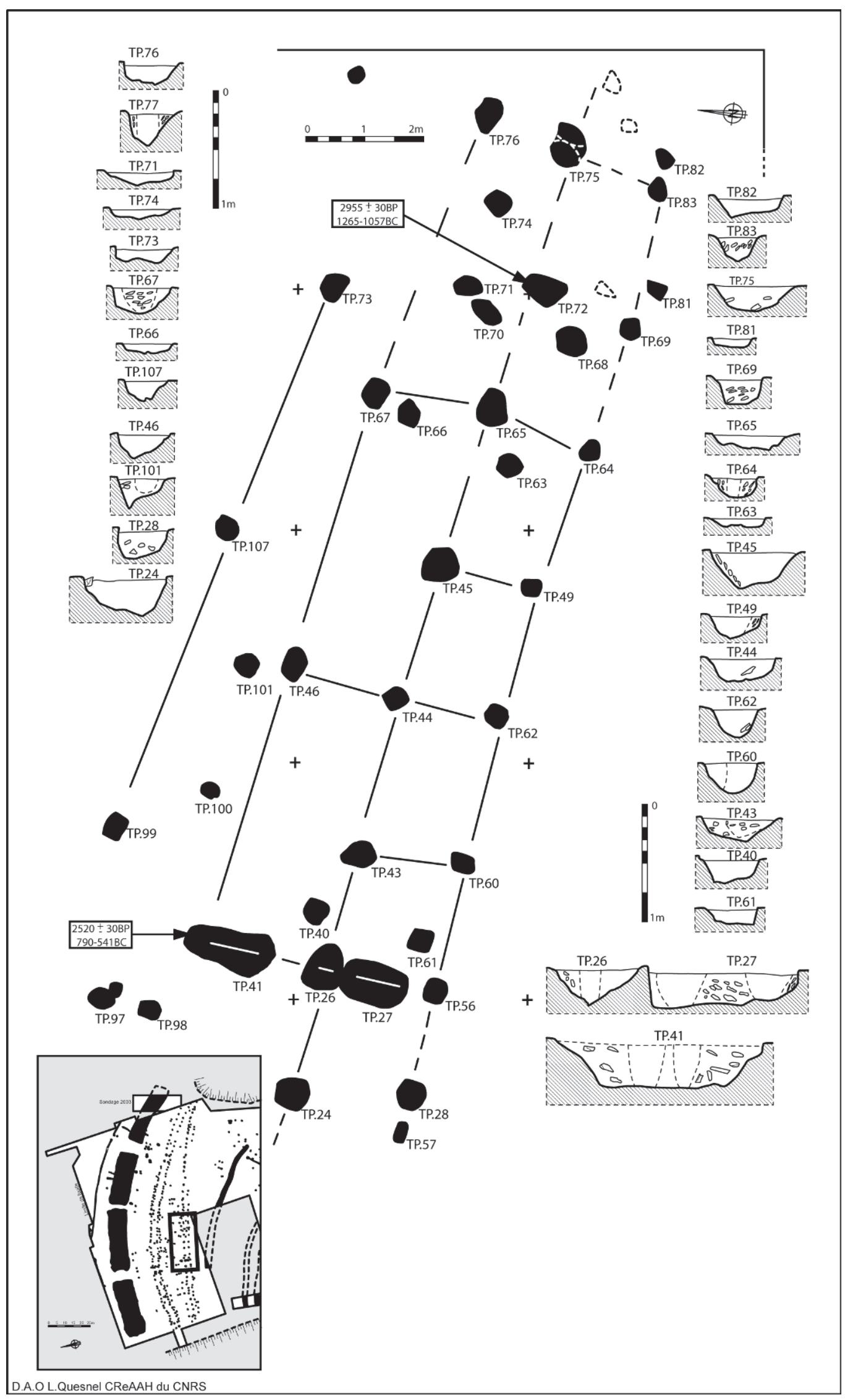


blement terreux plus sombre et plus organique. Reconnue sur une longueur de $104 \mathrm{~m}$, cette palissade barre également la totalité du promontoire mais son tracé est plus irrégulier que celui de l'ensemble précédent. Ces fondations sont creusées dans un secteur de schiste sain, perturbé dans sa partie orientale par un large filon de quartz et un fossé de parcellaire moderne qui expliquent certaines lacunes dans la continuité. La forme allongée de certaines des fondations indique clairement la présence de deux creusements jointifs, sans qu'on puisse distinguer entre l'implantation simultanée de deux poteaux mitoyens et une réfection de la palissade.

Sur sa moitié ouest, cet ensemble est bordé, à $2 \mathrm{~m}$ de part et d'autre, par deux alignements parallèles de poteaux dont la fonction est difficile à interpréter. S'il s'agit de palissades complémentaires, leur rapport avec la structure principale reste énigmatique. L'hypothèse d'un long bâtiment à deux ou trois nefs $(18 \times 7 \mathrm{~m}$ ?) n'est guère plus satisfaisante, même en présence des grandes fosses transversales TP.26, 27 et 41 pouvant figurer un éventuel pignon (fig. 28). La proposition de petits appentis (greniers?) de $2 \mathrm{~m}$, de large et adossés de part et d'autre de la palissade, semble plus raisonnable. Dans ce cas, les grandes fosses transversales précitées pourraient border une entrée dans la nouvelle zone enclose, implantée dans l'axe d'une interruption du fossé $2 \mathrm{~b} .1 / 2 \mathrm{~b} .2$, antérieur de quelques siècles mais constituant certainement encore un obstacle (voir supra, $\$$ VII). L'interprétation comme greniers peut être proposée également pour les éléments identiques adossés en partie interne dans les secteurs central et oriental de la palissade principale (fig. 16). Le doublement des poteaux de celle-ci correspond dans certains cas à l'implantation de ces petits bâtiments.

Deux datations radiocarbone rattachent cet ensemble au premier âge du Fer : pour le secteur ouest, TP.41 Lyon4662 (SacA-9813) : $2520 \pm 30$ BP, soit 790-541 av. J.-C. en âge calibré et, pour le secteur est, TP.528 : GrN-30618 : $2530 \pm 60 \mathrm{BP}$, soit $810-410 \mathrm{av}$. J.-C. Une troisième datation nous ramène à l'âge du Bronze : TP.72 : Lyon-4663 (Sac A-9814) : $2955 \pm 30$ BP, soit 1265-1057 av. J.-C. en âge calibré, mais il faut prendre en compte les risques de pollution à partir d'éléments de l'occupation antérieure localisée à proximité immédiate. Bien entendu, cette remarque est également valable pour le mobilier dont l'attribution à l'une ou l'autre période devra être relativisée.

Une date du premier âge du Fer (Hallstatt $\mathrm{C}$ ), très proche des deux précédentes, est également fournie par un foyer (fo.09) recoupant le bord interne du tronçon oriental du fossé segmenté $2 \mathrm{~b} .4$, à vingt mètres au nord de la palissade 3 (fig. 16) : GrN-30617: $2575 \pm 25$ BP, soit 810-750 avant J.-C. en âge calibré. Cette structure de combustion paraît avoir été installée en bordure du fossé à un stade de comblement avancé, probablement au pied des vestiges du rempart antérieur.

\section{B. Un large fossé de barrage de l'âge du Fer au sud}

En partie sud du site, le large fossé f5, mis en évidence très nettement par les anomalies phytologiques, barre le promontoire cinquante mètres avant sa pointe selon une orientation nord-ouest-sud-est (fig. 4 et fig. 29 à 31). Il fut dégagé sur la quasi totalité de sa longueur $(50 \mathrm{~m})$ lors du décapage 2006, à l'exception des extrémités, situées dans les bordures boisées du promontoire. Constitué de deux segments rectilignes de $25 \mathrm{~m}$ de longueur, son tracé marque un net infléchissement en partie centrale. Cet aspect facetté du barrage n'est pas sans rappeler celui des structures de l'âge du Bronze plus au nord. Ce fossé a fait l'objet de deux sondages de 2,50 m de large, le premier à l'ouest dans l'emprise du sondage D de 2003, le second au sud-est en 2006.

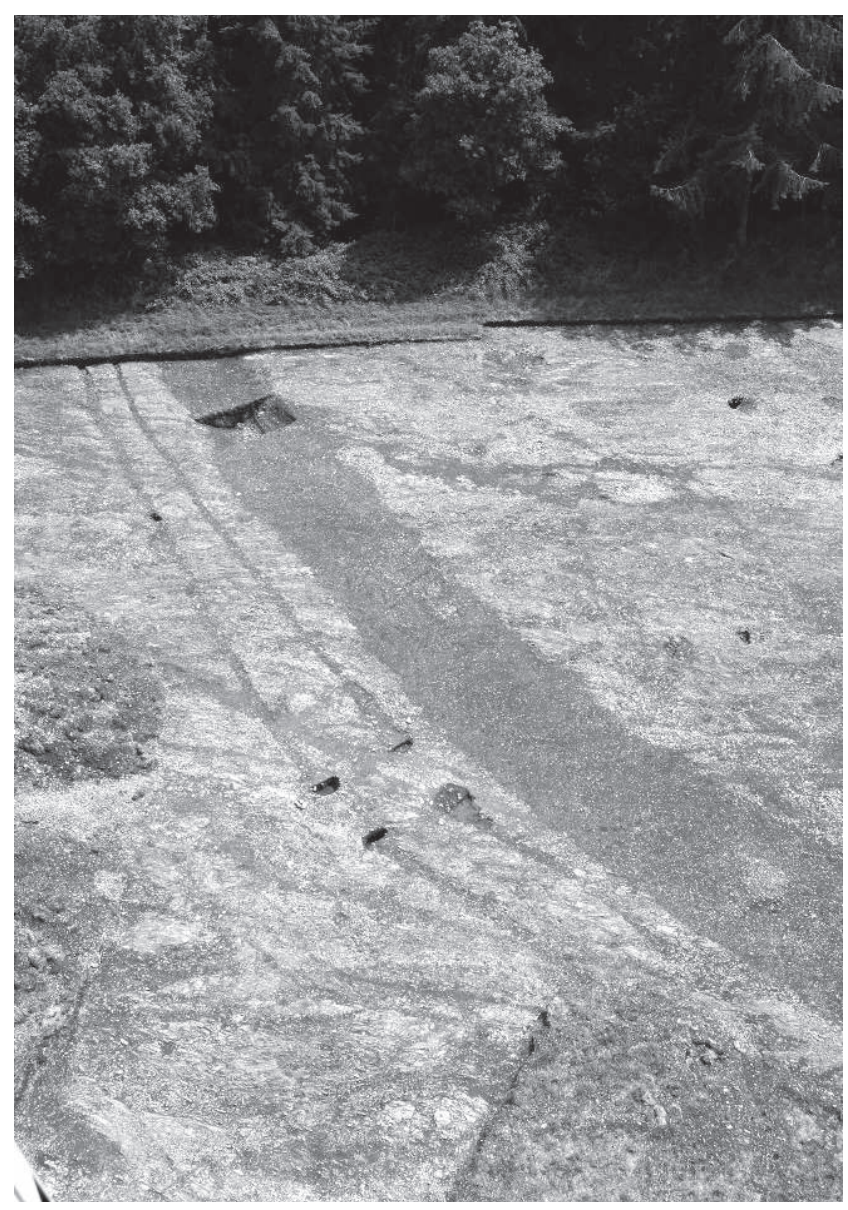

Figure 29 : Vue du nord-ouest du fossé 5 de l'âge du Fer et des deux tranchées de palissades parallèles fo. 25-26 et fo.27-28 avec entrées axiales, attribuées au Moyen Âge.

Figure 29: View from north-west of Iron Age ditch 5 and of two parallel palisade trenches fo. 25-26 and fo.27-28 with axial entrances, ascribed to Mediaeval period. 
Le creusement, à profil en $\mathrm{V}$ régulier, est large de 3,50 à 4,40 $\mathrm{m}$ et profond de 2,20 $\mathrm{m}$ dans le substrat. Dans le sondage sud-est (fig. 30, 31), la coupe CD (nord-ouest) présente le profil en $\mathrm{V}$ bien marqué, prolongé par une goulotte axiale jusqu'à $2,20 \mathrm{~m}$ de profondeur; en revanche, sur la coupe EF (sud-est), le profil est plus adouci et la profondeur réduite à $1,20 \mathrm{~m}$. Cette différence entre les coupes s'explique par une remontée du fond et une réduction progressive de la profondeur dont l'inclinaison permet d'estimer l'extrémité du fossé entre cinq et six mètres du sondage (en secteur non accessible à la fouille). Cela laisserait ainsi un passage d'environ cinq à six mètres entre ce dernier et la pente sud du promontoire. Les coupes révèlent un comblement en deux phases principales (fig. 30, coupe CD). Le fond étroit est comblé (US.1), sur une épaisseur d'un mètre environ, par un amas de plaquettes de schiste de tailles diverses avec peu de terre interstitielle. Cette couche de base est scellée par deux lentilles de terre brun-gris foncé, grasse et sans plaquettes (US.2 et US.3); un amas de schiste brun jaune (US.4) vient s'intercaler entre les deux en partie sud et semble provenir de la dégradation du bord interne du fossé. Ces trois lentilles marquent un arrêt du comblement et la formation d'un sol sur un profil d'équilibre du barrage; ce niveau est marqué d'un lit de charbons de bois dans la partie sud du sondage, indice probable d'un brûlis. Le pendage de ces couches montre un remplissage à partir de la zone interne, ce qui laisse supposer l'existence d'un talus en bordure interne.

À ce stade, un dénivelé de plus de $1,50 \mathrm{~m}$ est conservé et représente un obstacle à prendre en considération dans les critères d'implantation des occupations ultérieures de ce secteur au Moyen Âge. Les deux couches à profil en berceau achevant de combler le fossé sont nettement plus humiques, mêlant quelques fragments de schiste dans une matrice brun-gris foncé pour l'US. 5 et une terre végétale brun foncé pour l'US.6 sommitale dont on peut faire remonter l'origine aux derniers travaux de remembrements agricoles des années 1960.

Une datation radiocarbone a été obtenue sur un échantillon de charbon provenant du fond du sondage sud-est (US.1) : f5 : Lyon-4664 (SacA-9815) : $2605 \pm 30$ BP, soit 830-670 av. J.-C. en âge calibré. Elle s'accorde avec celles obtenues sur la palissade (ensemble 3) localisée à plus de $80 \mathrm{~m}$ au nord. Par ailleurs le mobilier mis au jour dans les deux sondages confirme l'attribution chronologique à l'âge du Fer.

Paradoxalement, aucune structure contemporaine n'a été mise au jour dans l'espace restreint $\left(1600 \mathrm{~m}^{2}\right.$ environ de surface utile) enclos par cette fortification. C'est également le cas dans le vaste espace intermédiaire entre la palissade 3 et le fossé 5 (fig. 4 et 8), ce qui pose la question de la fonction de ces ensembles. La palissade en elle-même et ses petits bâtiments annexes pourraient soutenir l'hypothèse d'un ouvrage agricole (enclos à bestiaux?) dépendant d'un établissement proche plus conséquent. La puissance du barrage sud (fossé 5 et son talus associé) semble relever d'un autre statut et rompt par sa morphologie et son emprise réduite avec les établissements habituellement connus pour cette période. On imagine mal qu'un tel investissement en travaux de terrassement ne protège que des structures suffisamment légères pour ne laisser aucune trace sur le substrat. L'hypothèse d'un site abandonné en cours de réalisation n'est pas à exclure : la perception du caractère inachevé d'aménagements est une notion de plus en plus prise en compte en archéologie. Pour expliquer cette absence apparente d'édifices dans l'enceinte de l'âge du Fer, la construction sur sablières basses ne laissant pas de traces sur le sous-sol pourrait aussi être évoquée, mais aucune concentration de mobilier ne vient étayer cette hypothèse.

Parallèlement au bord nord du fossé 5 , deux petites tranchées de palissade (fo. 25 à 28 , fig. 29 et 30 ) marquent une interruption pour former une large entrée centrale bordée de quatre trous de poteau. Bien que l'un de ces trous (TP.494) ait fourni une datation au second âge du Fer (GrN 30623 : $2240 \pm 30$ BP, soit 400-200 av. J.-C. en âge calibré), la présence de mobilier plus récent dans ces fondations légères et leur position relativement aux structures voisines les rattachent à l'occupation du Moyen Âge (voir infra, $\$$ IX F). Ces deux palissades parallèles sont un exemple d'adaptation à la présence de traces pérennes d'aménagements plus anciens comme le fossé 5 .

\section{C. Le mobilier de l'ensemble de l'âge du Fer}

Ce matériel provient de trois contextes complémentaires : les dernières phases de comblement (US.2 et US.3) du fossé segmenté $2 \mathrm{~b}$, les trous de poteau et l'emprise de l'ensemble palissadé 3 au nord et le grand fossé 5 au sud (fig. 21 et infra, fig. 50). Ces différentes structures ont pu piéger des éléments antérieurs, notamment dans le secteur nord aménagé au Bronze final, et certains mélanges ne sont pas à exclure.

Un total de 132 tessons ou lots de tessons d'un même vase (quatre au maximum) ont été recensés pour un poids de 2520 g. Certains caractères généraux distinguent cette série de la précédente attribuée essentiellement au Bronze moyen ou final (cf. infra, fig. 51). Le morcellement et l'usure sont moins accentués avec $57 \%$ de pièces d'une surface inférieure à $4 \mathrm{~cm}^{2}$ et $58 \%$ aux contours érodés. Les pâtes à teinte foncée gris-noir sont mieux représentées (40\%). Les aménagements de surface des parois sont plus fréquents $(51,5 \%)$ et le lustré sur engobe de plusieurs tessons est caractéristique des céramiques de qualité de l'âge du Fer. De même, pour $44 \%$ des tessons, le dégraissant se caracté- 


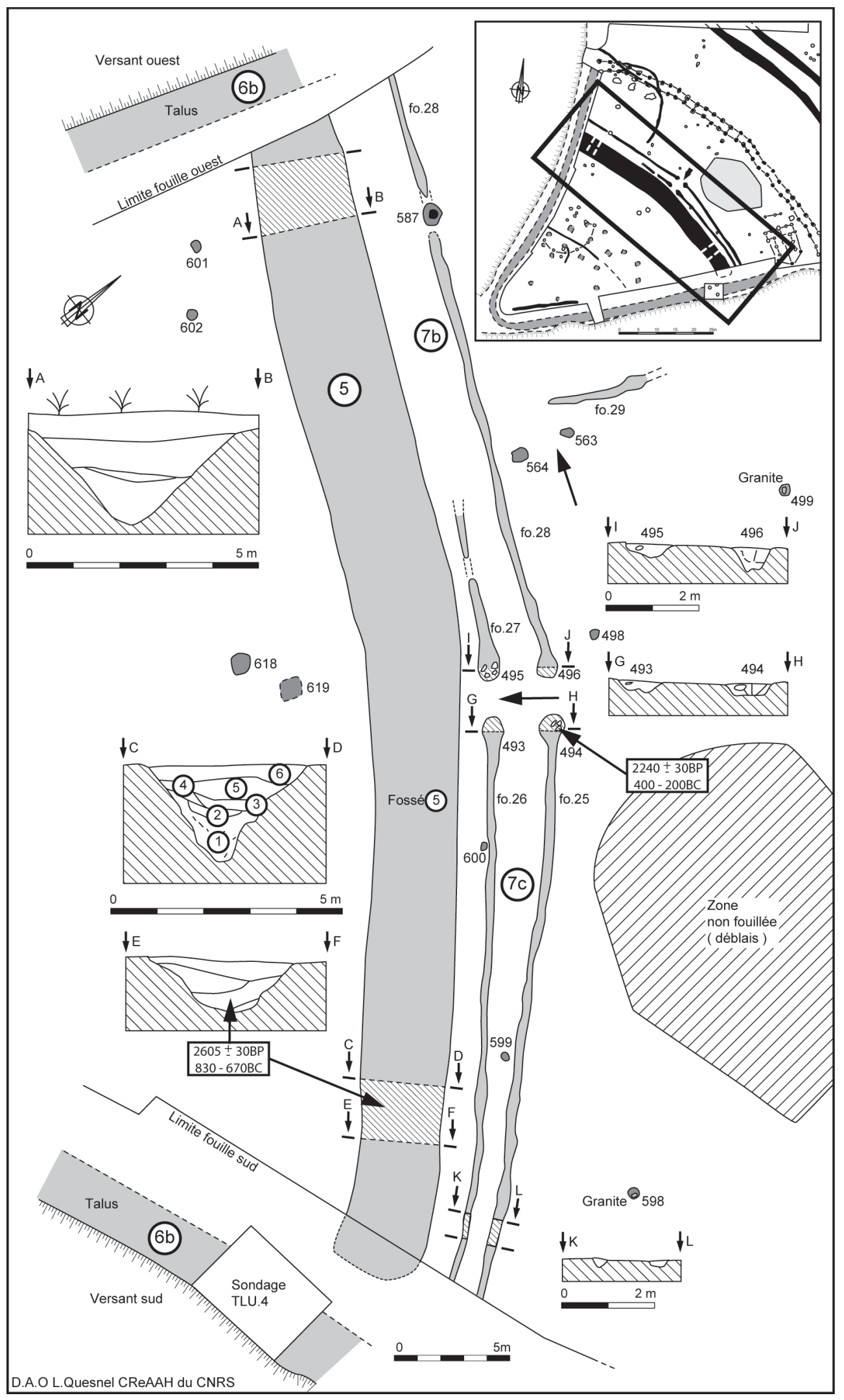

Figure 30 : Fossé de barrage 5 (âge du Fer) en zone sud, coupes $\mathrm{CD}$ et EF du sondage sud-est; tranchées de fondation parallèles fo. 25 à 28 ; coupes associées et datations $14 \mathrm{C}$ en encadrés. Figure 30: Iron age ditch 5 in South sector and parallel palisade trenches fo. 25 to 28 ; associated sections and radiocarbon datings in inserts. 


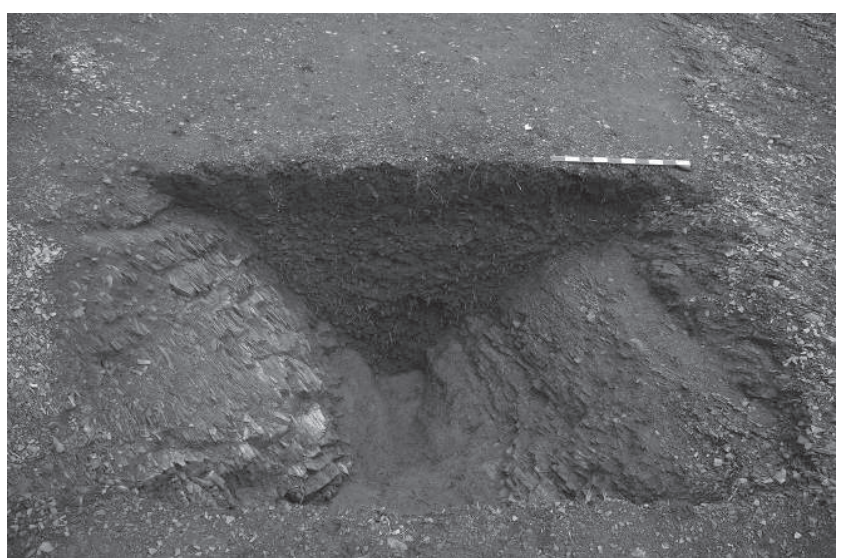

Figure 31 : Vue de la coupe CD du fossé 5 à profil en V (sondage sud).

Figure 31: View of $C D$ section of the $V$ shaped ditch 5 (south sondage).

rise par sa finesse et son calibrage. Enfin, la dureté des pâtes, pour $42 \%$ des éléments, indique une cuisson parfaitement maîtrisée, qualité qui explique également une meilleure résistance au morcellement et à l'érosion par rapport à la série précédente.

L'absence de céramique archéologiquement complète est faiblement compensée par quelques éléments de forme : cinq fragments de lèvres, quatre carènes, six fonds plats sans débord et un décor (fig. 23 et infra, fig. 51). Ce seul décor est issu du TP.27 du secteur de l'ensemble palissadé 3 (fig. 23, n 16). C'est une série d'entailles obliques réalisées à la spatule sur le bord interne d'un vase à pâte grise; la lèvre simple est légèrement aplatie et le col concave. Il peut être comparé aux tessons incisés sur le bord interne de la lèvre mis au jour d'une part dans des niveaux postérieurs à l'âge du Bronze à Mez-Notariou, Ouessant (Le Bihan [dir.], 2010, p. 284, fig. 102 et pl. 183, n 11-105) et d'autre part dans l'enclos de Kerlande à Brandivy (Lecornec, 1973, fig. 3, $\mathrm{n}^{\circ} 2$; voir supra, $\$$. VII F).

Le fossé 5 , au sud, a livré plusieurs tessons d'un vase à engobe noir lissé à forte courbure de la panse et un fragment de lèvre simple d'un récipient de qualité à large ouverture (fig. 23, n 18 et 19). Cette bonne qualité de pâte et de façonnage se retrouve sur un fragment de fond plat à pied régulièrement arrondi issu du fossé $2 \mathrm{~b} .1$, sondage 4 , US. 2 $\left(\mathrm{n}^{\circ} 20\right)$. De ce même fossé (sondage 1) proviennent les trois fragments d'une fusaïole cylindrique (hauteur $22 \mathrm{~mm}$, diamètre $25 \mathrm{~mm}$ ), en terre cuite orange clair $\left(\mathrm{n}^{\circ} 9\right)$. Une seconde fusaïole biconique en terre cuite est issue de la tranchée fo. 25 au sud (cf. infra, fig. 51, n 7 ).

\section{D. Éléments de comparaison pour l'âge du Fer}

Les éperons barrés occupés ou réoccupés à l'âge du Fer sont nombreux en Bretagne, sur les côtes et dans les îles en particulier, à la faveur des nombreux promontoires sculptés par la mer, mais peu d'entre-eux ont fait l'objet d'études récentes. À La Rochette, le fossé 5 ceinturant la pointe de l'éperon n'est pas sans rappeler celui de Beaumont à SaintLaurent-sur-Oust (Morbihan) sur lequel, après une occupation au Néolithique final, le second âge du Fer a laissé son empreinte sous la forme d'un fossé de barrage délimitant environ un hectare jusqu'à la pointe du promontoire et des aménagements en terrasses en sommet de pente sud (Tinévez, 1992).

En position stratégique au nord de l'estuaire de la Loire, la pointe de Penchâteau au Pouliguen (Loire-Atlantique) a été fortifiée et habitée pendant toute la durée de l'âge du Fer. Une évaluation archéologique réalisée en 1991 (Gaiffe et al., 1995) y mit en évidence un imposant système défensif composé d'une succession de fossés doublés de talus délimitant un espace d'environ sept hectares. Mais dans ce cas, la fonction d'habitat interne est attestée par la présence de structures et d'un mobilier dense associé.

Dans les régions voisines, les exemples de promontoires dont l'occupation durant l'âge du Fer se rétracte vers la pointe de l'éperon par rapport aux occupations antérieures ne sont pas rares. Ainsi, à La Campagne à Basly (Calvados), une première palissade datée du Néolithique final enclot une emprise de 2,5 hectares, tandis qu'au Hallstatt final, seule la pointe est barrée d'un puissant fossé doublé d'un talus muni d'une entrée axiale, la nouvelle surface occupée ne dépassant pas $4000 \mathrm{~m}^{2}$ (San Juan et al., 2000; 2007). Le même phénomène caractérise le Camp Allaric à Aslonnes (Vienne), déjà évoqué pour l'âge du Bronze, où la surface enclose se réduit de deux hectares au Bronze final à $2500 \mathrm{~m}^{2}$ au second âge du Fer (Pautreau et Maitay, 2007).

\section{UNE FORTIFICATION DU HAUT MOYEN ÂGE À LA POINTE DU PROMONTOIRE (J.-Y. T., J. V. D. P.)}

\section{A. Une double palissade barrant la pointe du promontoire (ensemble 6a)}

Au sud du site également, à $75 \mathrm{~m}$ de la pointe, une double palissade (6a) est profondément ancrée dans le substrat (fig. 4 et 32 à 39). Un total de soixante trous de poteau a été mis au jour, répartis en deux files présentant un écartement régulier de $1,50 \mathrm{~m}$, les trente paires de poteaux étant dis- 


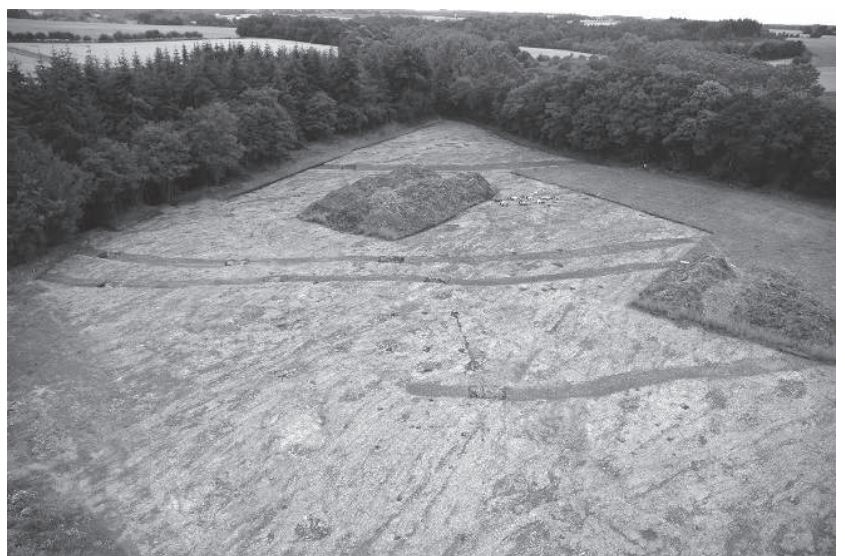

Figure 32 : Vue, vers le sud, de la pointe du promontoire en juin 2006. Le tas de déblais central et la berme sud ouest seront par la suite réduits pour permettre un dégagement complet de la double palissade du haut Moyen Âge, en cours de fouille sur la photographie.

Figure 32: Southwards view of the promontory point in june 2006. The central rubble heap and the south-west berm will be reduced later for complete study of the early Mediaeval double palisade, the excavation of which is in progress on the photograph.

tantes l'une de l'autre de 2 à $2,5 \mathrm{~m}$. À l'instar des ensembles linéaires décrits précédemment, le tracé général de cet ouvrage n'est pas parfaitement arqué, mais plutôt légèrement sinueux, en éléments rectilignes successifs. Il assure un obstacle continu sur une longueur de plus de $80 \mathrm{~m}$ et s'interrompt environ $5 \mathrm{~m}$ avant les ruptures de pente de part et d'autre de l'éperon.

L'emplacement d'une entrée ne peut être clairement définie, cependant le rythme des paires de poteaux varie par endroits. Ainsi, à $10 \mathrm{~m}$ de l'extrémité sud-est, un écartement de $3 \mathrm{~m}$ (au lieu des 2,50 $\mathrm{m}$ habituels) sépare les paires 446449 et 447-448 et, face au bâtiment intérieur, on pourrait y voir une entrée dans l'enceinte, complétée par l'alignement des poteaux 452, 595 et 596, devant la façade principale de la maison (fig. 34). Une disposition similaire est repérable près de l'extrémité nord-ouest (paires 569-570 et 572-573), à $10 \mathrm{~m}$ de l'extrémité comme au sud-est. Nous aurions ainsi une seconde entrée disposée symétriquement (fig. 37).

A contrario, une réduction de l'écartement type à $1,50 \mathrm{~m}$ pour trois paires $(551-552,553-556$ et 557-558) en zone centrale pourrait trahir cette fois la présence d'une structure dont le faible écartement des éléments porteurs indiquerait une élévation supérieure, à l'image d'une tour de guet (fig. 39).

La fouille d'une douzaine de ces trous de poteau montre les dimensions importantes des creusements $(0,80$ à $1 \mathrm{~m}$ de diamètre, 0,80 à $1 \mathrm{~m}$ de profondeur dans le schiste); en raison de la nature diaclasée du substrat, les trous ont

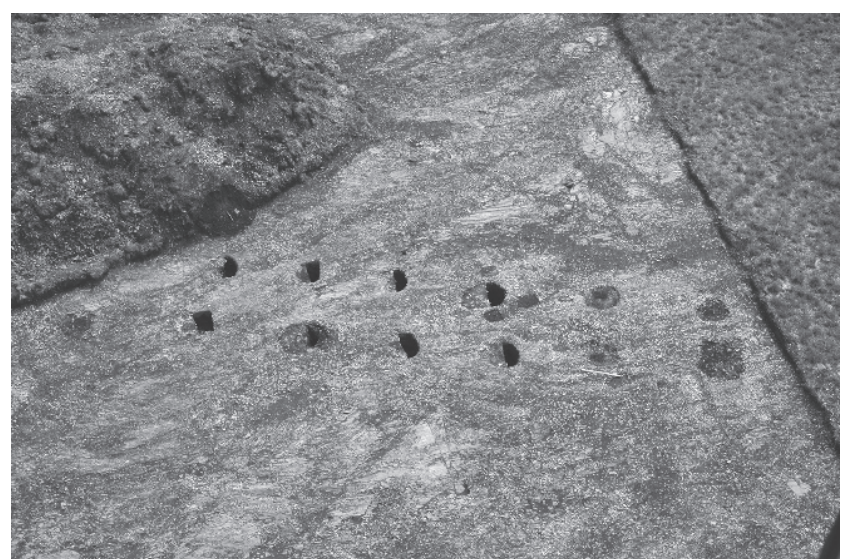

Figure 33 : Vue partielle de la double palissade du haut Moyen Âge (6a) en juin 2006 (son dégagement complet sera réalisé en septembre 2006).

Figure 33: Partial view of the early Mediaeval double palissade (6a) in june 2006; its complete clearance would be achieved in september 2006.

des profils très anguleux, avec parois verticales ou presque et fonds plats. Les comblements sont exclusivement composés du schiste extrait et se révèlent très difficiles à détecter au décapage malgré leurs dimensions importantes. En revanche, les poteaux sont nettement visibles par contraste de couleur dans le comblement car ils sont systématiquement incendiés (fig. 35, 36, 38). Certains présentent un aspect noir très charbonneux, $d$ 'autres une couleur orangé clair en raison de la présence de sédiment et plaquettes de schiste rubéfiés; ces derniers signalent l'effondrement d'un noyau pierreux rubéfié dans le négatif des poteaux, qui sont presque tous équarris et de section rectangulaire calibrée, de $30-40$ sur $25-30 \mathrm{~cm}$ en moyenne. Leur orientation est préférentiellement dans l'axe des palissades. Pour un seul cas (TP.487), la trace du poteau est estompée et semble signaler un arrachage.

Signalons la présence de trois zones cendreuses de forme irrégulière ( 2 x $1 \mathrm{~m}$ environ), localisées à même le substrat le long de la palissade interne près de son extrémité occidentale (fig. 37); sans qu'ils soient datés, le lien de ces amas cendreux avec l'incendie peut être évoqué en raison de leur disposition régulière près de l'enceinte, soit en amont comme cause du sinistre, soit comme résultat de l'effondrement de la structure. Leur position stratigraphique au contact du substrat semble indiquer un décapage du sol en préalable à l'édification de l'enceinte. Ce constat corroborerait la quasi absence de terre végétale dans le comblement des fondations.

Deux datations radiocarbone ont été obtenues sur charbons issus de deux poteaux : TP.481 GrN-30621 : $1285 \pm$ 


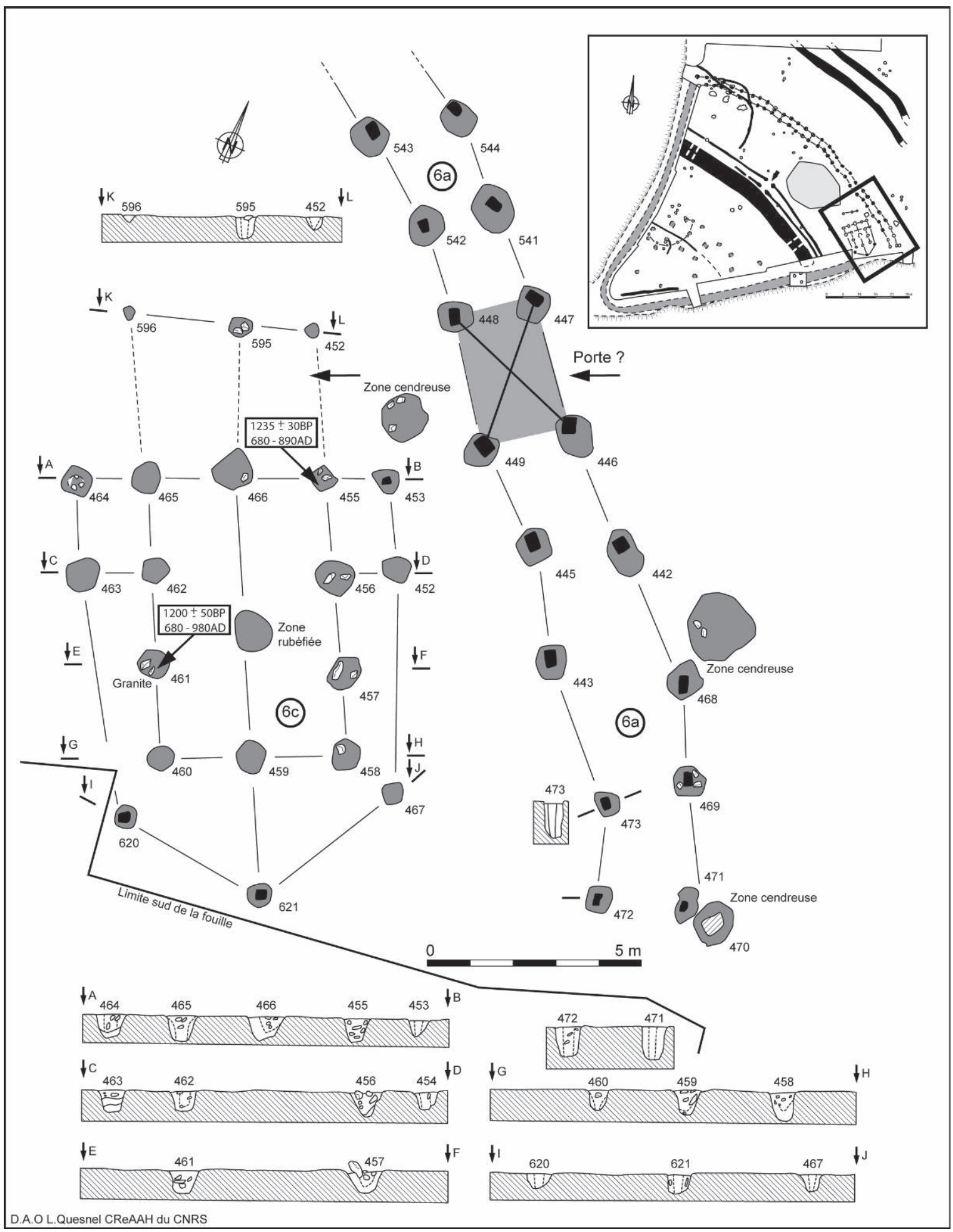

Figure 34 : Secteur sud-est de l'enceinte incendiée du haut Moyen Âge : double palissade 6a à poteaux équarris et bâtiment $6 \mathrm{c}$ sur poteaux à foyer central. L'hypothèse d'une entrée sud-est dans l'enceinte à hauteur du bâtiment est proposée en raison d'un écartement supérieur des poteaux 446-449.

Figure 34: South sector of the early Mediaeval enclosure destroyed by fire: double palisade 6a with squared timber and post-built house $6 c$ with central hearth. An entrance at the south-east of the enclosure, facing the house, is proposed as an hypothesis due to a larger spacing between the posts 446-449. 


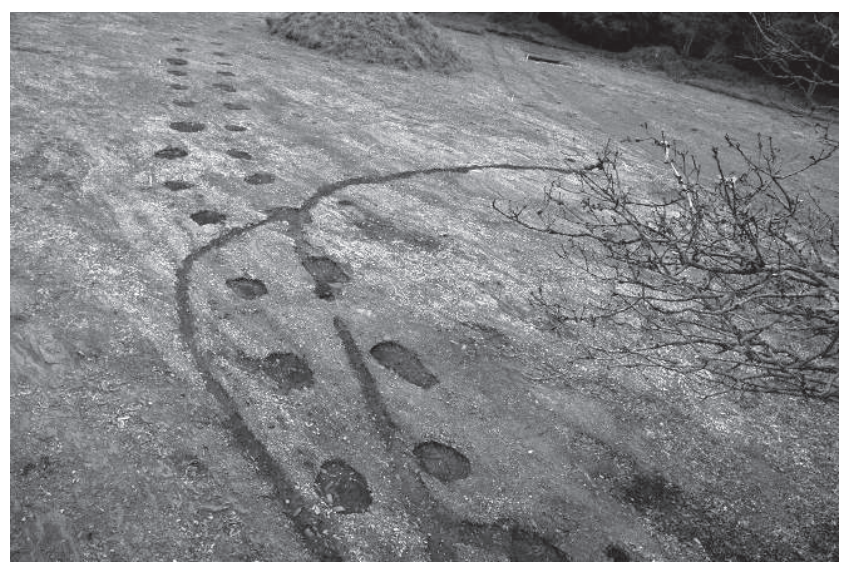

Figure 35 : Vue de l'ouest de la double palissade 6a du haut Moyen Âge recoupée par l'enclos curvilinéaire $6 \mathrm{~b}$ du plein Moyen Âge. Les poteaux équarris et incendiés sont visibles (en noir et orange clair). Le long de la palissade interne, trois zones cendreuses à même le substrat sont également visibles.

Figure 35: View from the east of the early Mediaeval double palissade $6 a$, cross-cut by the curvilinear mediaeval enclosure $6 b$. The squared burnt posts can be seen (in black or orange). Along the internal palisade, three ashy area can be seen directly on the substratum.

20 BP, soit 675-780 apr. J.-C. en âge calibré et TP.483 : GrN-30622 : $1290 \pm 20$ BP, soit 660-780 apr. J.-C. en âge calibré.

\section{B. Un bloc de granite dressé}

À $5 \mathrm{~m}$ au sud de la partie médiane de la double palissade 6a, un bloc de granite brisé a été mis au jour lors du décapage de la terre végétale (fig. 37, structure 499; fig. 40). La fouille a mis en évidence l'ancrage soigné du bloc dans un trou à parois abruptes et fond plat de $0,40 \mathrm{~m}$ de diamètre, creusé dans le schiste sur $0,30 \mathrm{~m}$ de profondeur. Le bloc est conforté par un solide calage vertical comportant une plaque de schiste de 0,30 m de long et quelques plaquettes complémentaires dont le volume exclut l'hypothèse d'un calage de poteau. D'une hauteur conservée de 0,18 $\mathrm{m}$, le bloc de granite à gros grains n'émergeait que d'une dizaine de centimètres du niveau du substrat lors de sa découverte, probablement tronqué par les travaux agricoles. De base plane, sa section est plano-convexe et le bord arrondi semble travaillé par piquetage. Aucun élément de datation ne permet de rattacher ce bloc exogène et intentionnellement calé à l'une ou l'autre des occupations du promontoire. Sa fonction reste également indéterminée : ébauche de meule réutilisée, base de stèle ou de borne?

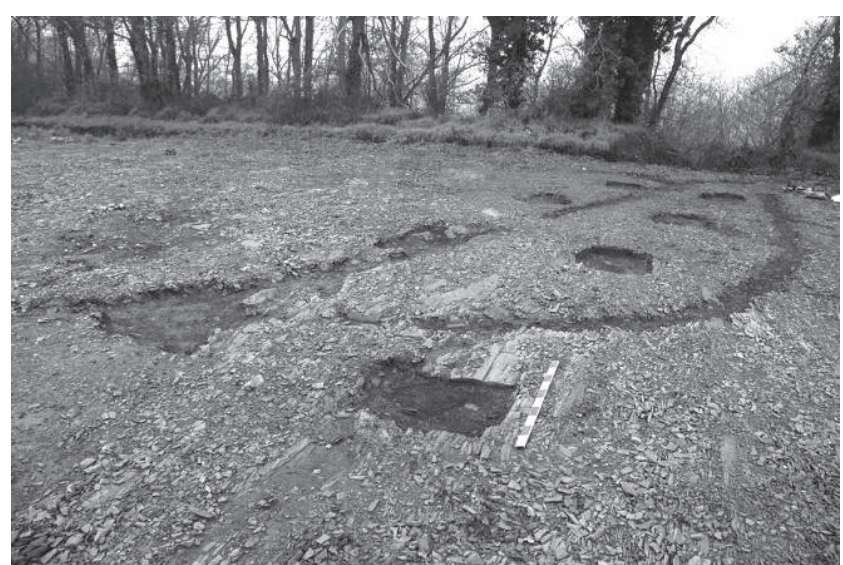

Figure 36 : Vue vers l'ouest de la double palissade du haut Moyen Âge qui s'incurve en arrière-plan pour se poursuivre sous le talus bordant la pointe du promontoire.

Figure 36: Westwards view of the early Mediaeval palisade which curves in background to go on under the boundary bank of the promontory.

\section{C. Un talus bas en bordure du promontoire}

Cette double structure se prolonge, en bordure de promontoire et jusqu'à la pointe, par un talus bas (6b), d'un mètre de hauteur maximum et en grande partie masqué par le couvert forestier en lisière des pentes (fig. 39 et 41 à 44). Cependant, déjà avant la fouille, la partie sud de ce talus (dénudée de toute végétation) se présentait sous la forme d'une masse de plaquettes de schiste rubéfiées. En fonction des secteurs accessibles sous le couvert forestier, trois sondages (TLU.2, TLU.3 et TLU.4) ont été réalisés sur son tracé. TLU.2 est localisé sur le versant ouest, dans le prolongement de la double palissade et met en évidence le lien direct entre ces deux structures $6 \mathrm{a}$ et $6 \mathrm{~b}$. TLU.3 et TLU.4 affectent le bord sud du promontoire (fig. 41 à 44). Les coupes obtenues révèlent une stratigraphie commune (fig. 41) : sous 0,10 à $0,40 \mathrm{~m}$ d'humus brun foncé, léger et poudreux (US.1), le noyau du talus est composé de terre pulvérulente orangé clair avec de nombreuses plaquettes et blocs de schiste rubéfiés (US.2). L'intensité de la chauffe s'exprime par la fusion et l'agrégation de certains blocs de schiste. La superposition de plaquettes de schiste, sur le versant externe notamment (coupe TLU2), semble signaler un vestige de parement sur les flancs du talus ou un effet de paroi d'un noyau pierreux dans une structure de bois. En TLU.2, l'US.2 recouvre la couche de base (US.3, épaisse de 0,10 à $0,20 \mathrm{~m}$ ), composée de terre brun foncé et petites plaquettes de schiste, reposant sur le substrat schisteux. Cette US.3 est recoupée par le trou de poteau 583 dont la présence a entraîné un affaissement de l'US.2 sus-jacente; 


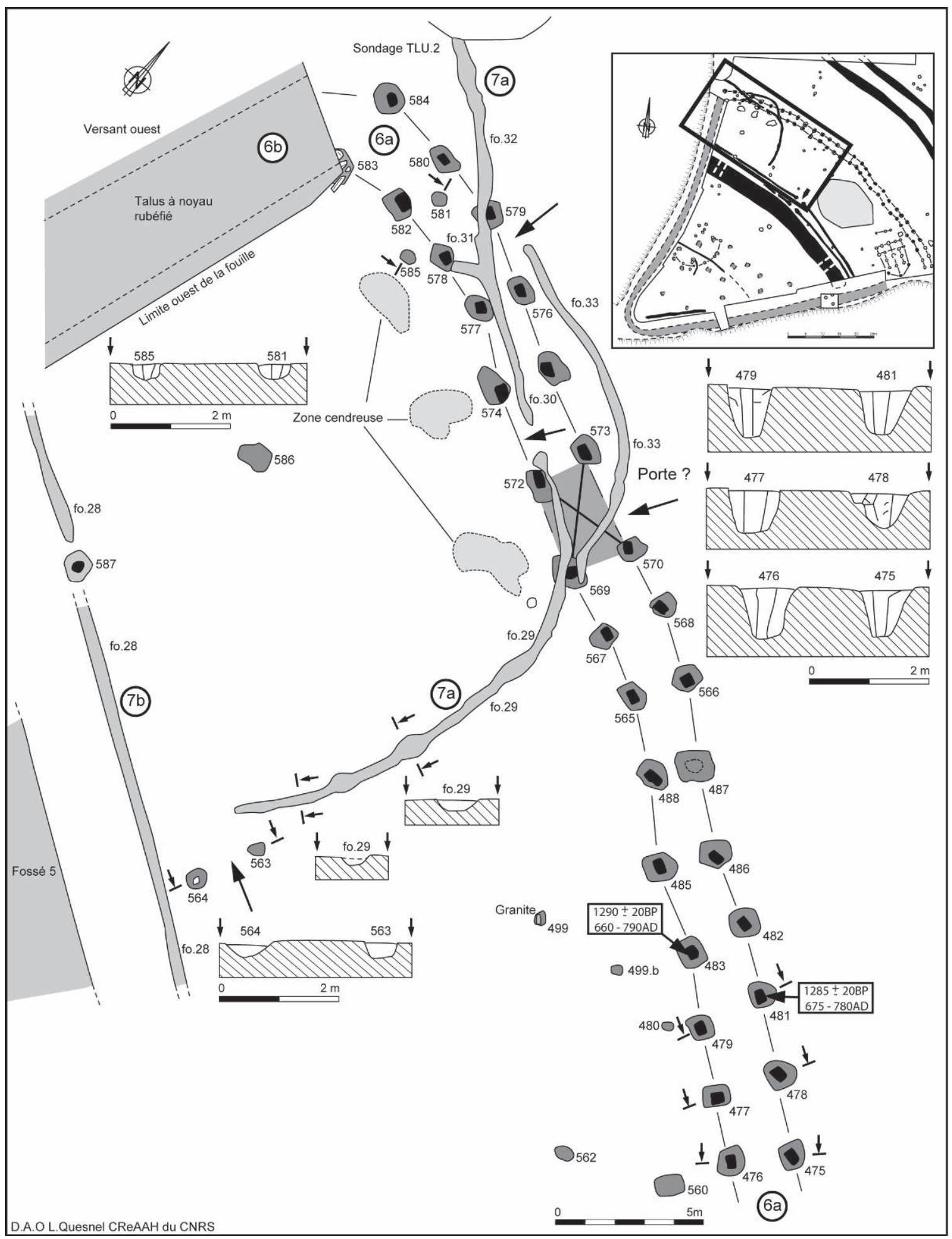

Figure 37 : Secteur nord-ouest de l'enceinte incendiée à poteaux équarris du haut Moyen Âge (6a), recoupée par la tranchée de fondation de l'enclos 7a. L'hypothèse d'une entrée dans l'enceinte est proposée en fonction de l'écartement plus important entre les TP.569, 570, 572 et 573 à l'image de l'extrémité sud-est. Le lien avec le talus 6b est assuré par le TP.583 scellé dessous. Un bloc de granite dressé est calé dans la structure 499.

Figure 37: North-west sector of the early Mediaeval enclosure 6a cutchecked by the foundation trench of enclosure 7 a. As in the south-east sector, an entrance is suggested between posts-holes TP.569-570 and 572-573, due to their larger spacing. The link with bank $6 b$ is evidenced by post- hole 583 sealed under it. A granite block is standing in hole 499. 


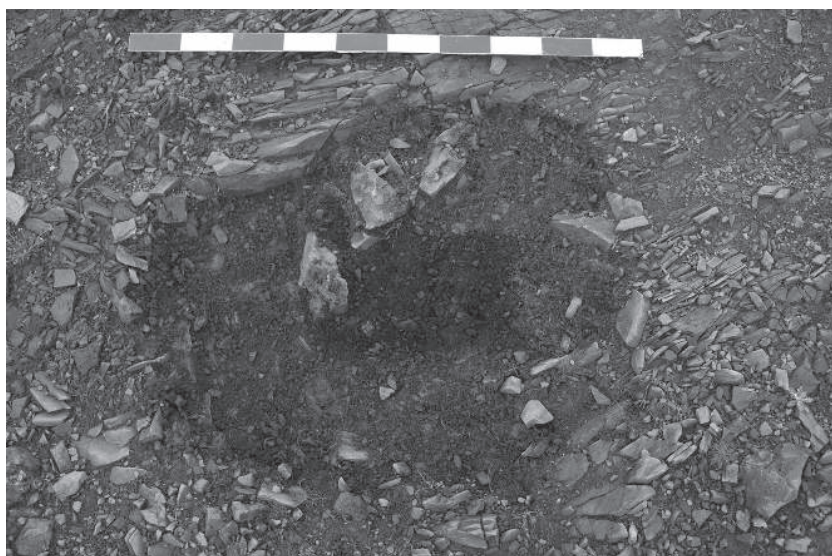

Figure 38 : Vues de détail des fondations de l'enceinte du haut Moyen Âge révélant la trace des poteaux équarris et systématiquement brûlés.

Figure 38: Detailed views of the early Mediaeval enclosure foundations showing the remains of squared posts with systematic burning.

le lien étroit entre les structures en poteaux plantés et le noyau pierreux calciné est ainsi attesté. En TLU.3, le noyau calciné repose sur une lentille charbonneuse, contenant de gros éléments de bois calciné dont l'un a fourni une datation radiocarbone: GrN-30624: $1280 \pm 20 \mathrm{BP}$, soit $680-780$ apr. J.-C. en âge calibré.

Provenant de cette coupe également, un gros fragment calciné d'une planche épaisse a été soumis à une étude dendrologique et, grâce à une très bonne conservation de l'échantillon (plus de 90 cernes, aubier conservé), la date de 682 après J.-C. a été obtenue et serait proche de la date d'abattage. Cette date correspond à la limite basse des marges statistiques des datations par le radiocarbone et situerait la construction du rempart à l'extrême fin du VII siècle de notre ère (analyse de Vincent Bernard).

En TLU.4, les observations ont été confirmées sur un sondage plus étendu (5 x $4 \mathrm{~m})$ (fig. 43, 44). Sous environ $0,10 \mathrm{~m}$ d'humus brun foncé (US.1), le noyau pierreux fortement rubéfié est entremêlé de poches charbonneuses et englobe à sa base plusieurs fragments de bois calcinés orientés est-ouest dans l'axe du talus. Ces éléments enchevêtrés reposent sur un lit de charbon de bois au contact direct avec le schiste du substrat, ce qui suppose un enlèvement des terres et la mise à nu du substrat avant l'effondrement (et probablement la construction) de la structure. Les éléments de bois ne présentent pas de façonnage particulier et semblent issus de grosses branches (longueurs conservées entre 0,50 et $1,20 \mathrm{~m}$; diamètre moyen $0,10-0,15 \mathrm{~m}$ ).

Après démontage de ces pièces, la fouille jusqu'au substrat a mis au jour trois trous de poteau de $0,40-0,50 \mathrm{~m}$ de profondeur et de diamètre (TP.1 à TP.3). Leur disposition par

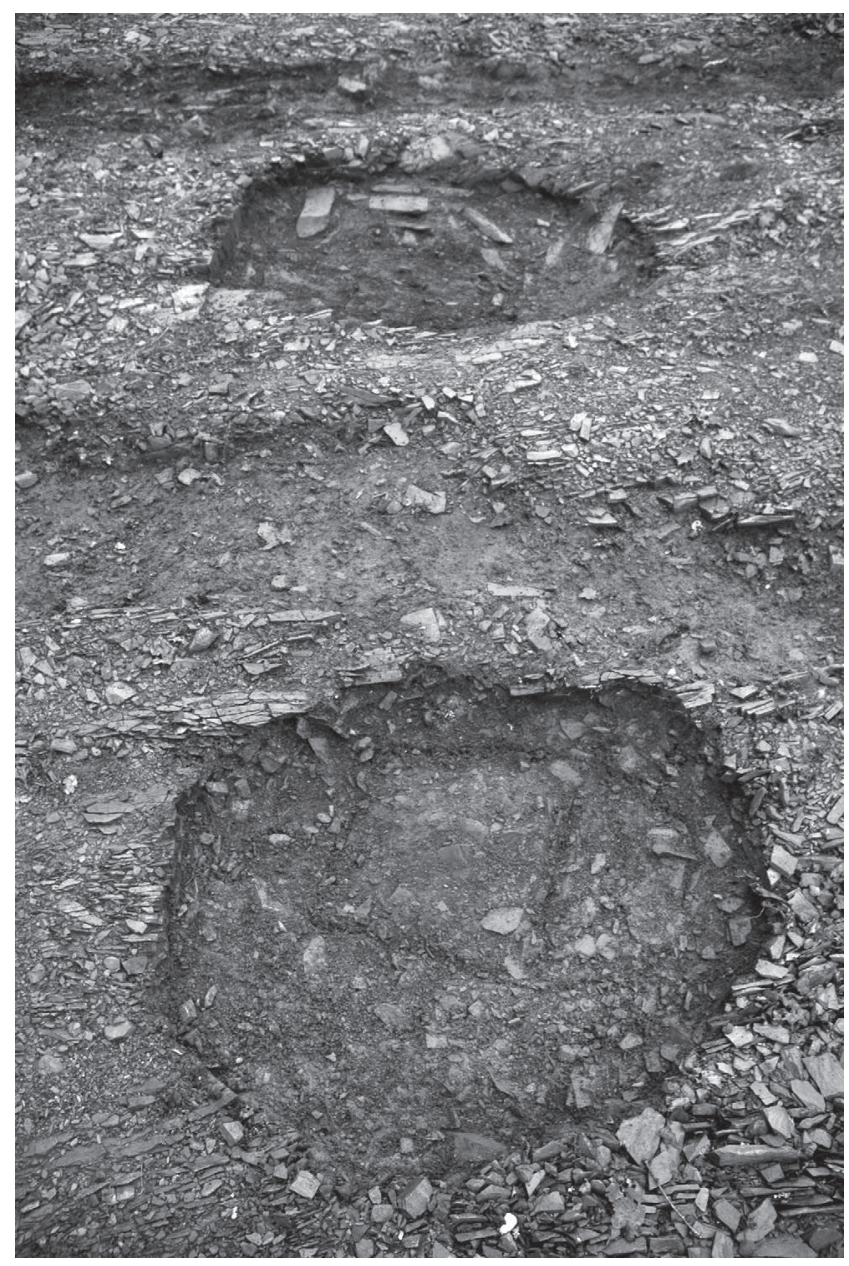

rapport à l'axe du talus rappelle celle de la double palissade 6a et l'écartement de $2 \mathrm{~m}$ entre TP. 1 et TP. 3 est identique. Le noyau pierreux rubéfié US.2 est visiblement localisé en bordure externe de la structure en bois, en sommet de rupture de pente. C'est également le cas sur la coupe TLU.2 sur laquelle l'US.2 rubéfiée présente un net effet de paroi vertical à $2 \mathrm{~m}$ du trou de poteau 583. L'hypothèse de la constitution d'un talus par décapage du sol végétal sur le tracé de l'enceinte et accumulation des déblais des fondations des poteaux est ainsi étayée, ce talus venant ensuite s'adosser à la palissade et la conforter à la base.

D'évidence, cette enceinte de bois et pierres ceinturant la pointe a subi une violente et totale destruction par incendie et la liaison entre la double palissade de barrage et le talus de bordure est incontestable, aucune trace de ce dernier n'étant visible au delà vers le nord ou l'est du promontoire. L'emprise enclose entre la double palissade et la pointe de l'éperon s'étend sur $3500 \mathrm{~m}^{2}$ environ (cf. infra, fig. 63). 


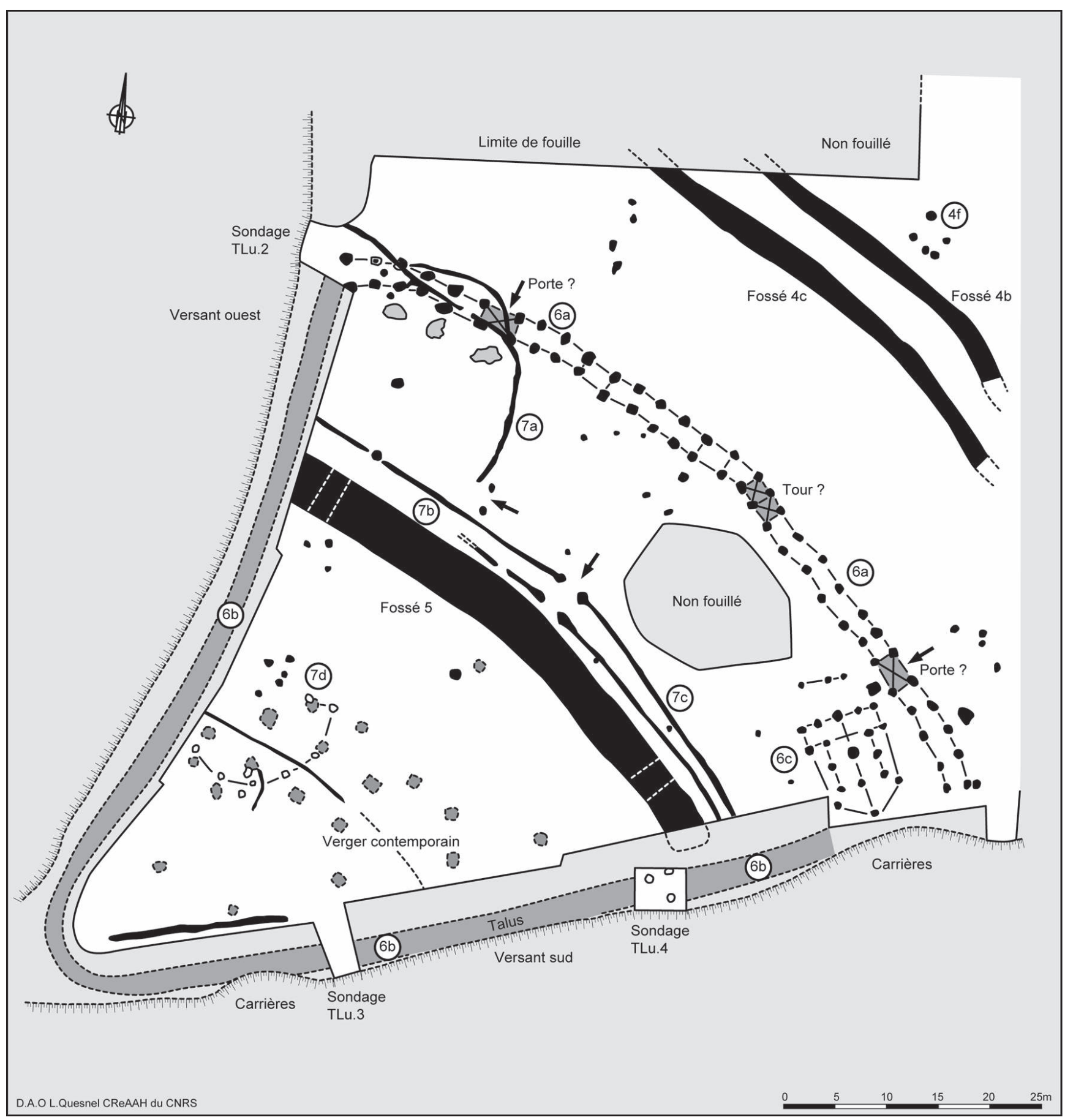

Figure 39 : Ensemble des structures mises au jour sur la pointe sud du promontoire : fossé 5, barrage de l'âge du Fer; ensemble 6 du haut Moyen Âge (6a double enceinte sur poteau, $6 \mathrm{~b}$ talus rubéfié en bordure du plateau, $6 c$ bâtiment sur poteaux porteurs à foyer central); ensemble 7, enclos du bas Moyen Âge (7a à 7c). En grisé, fosses de plantation d'un verger de la première moitié du $\mathrm{xx}^{\mathrm{e}}$ siècle dont les rangs en arcs de cercle se sont adaptés à la courbure de la fortification 5 de l'âge du Fer.

Figure 39: Structures discovered on the south point of the promontory. Iron Age ditch 5. Whole 6 of Early Mediaeval period (6a double post enclosure, $6 b$ burnt bank on promontory edge, 6c post-built house with central hearth). Whole 7, late Mediaeval enclosures (7a to $7 c$ ). In grey, $19^{\text {th }}$ century orchard tree holes, the arched disposition of which is adapted to the curvature of Iron Age fortification 5. 


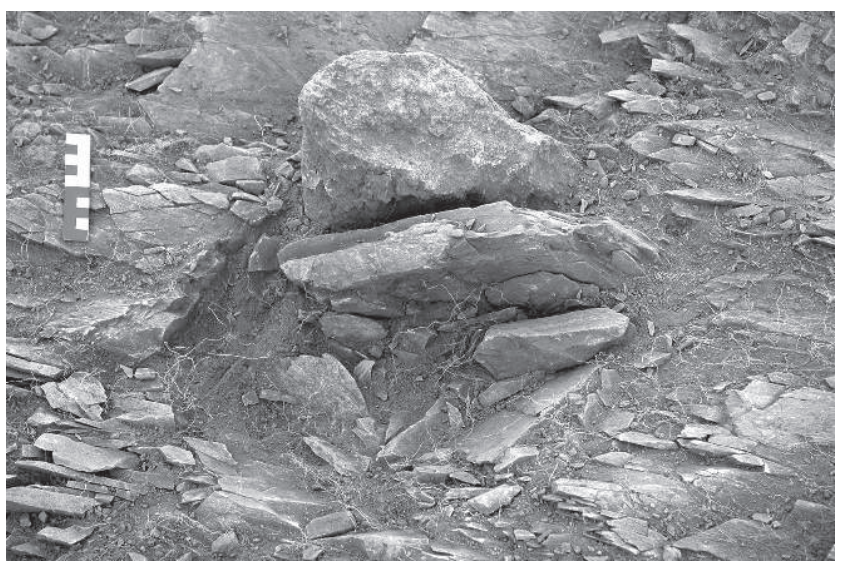

Figure 40 : Vue du bloc de granite calé dans le trou 499 par une petite dalle de schiste.

Figure 40: The granite block standing in hole 499 against a small schist packing slab.

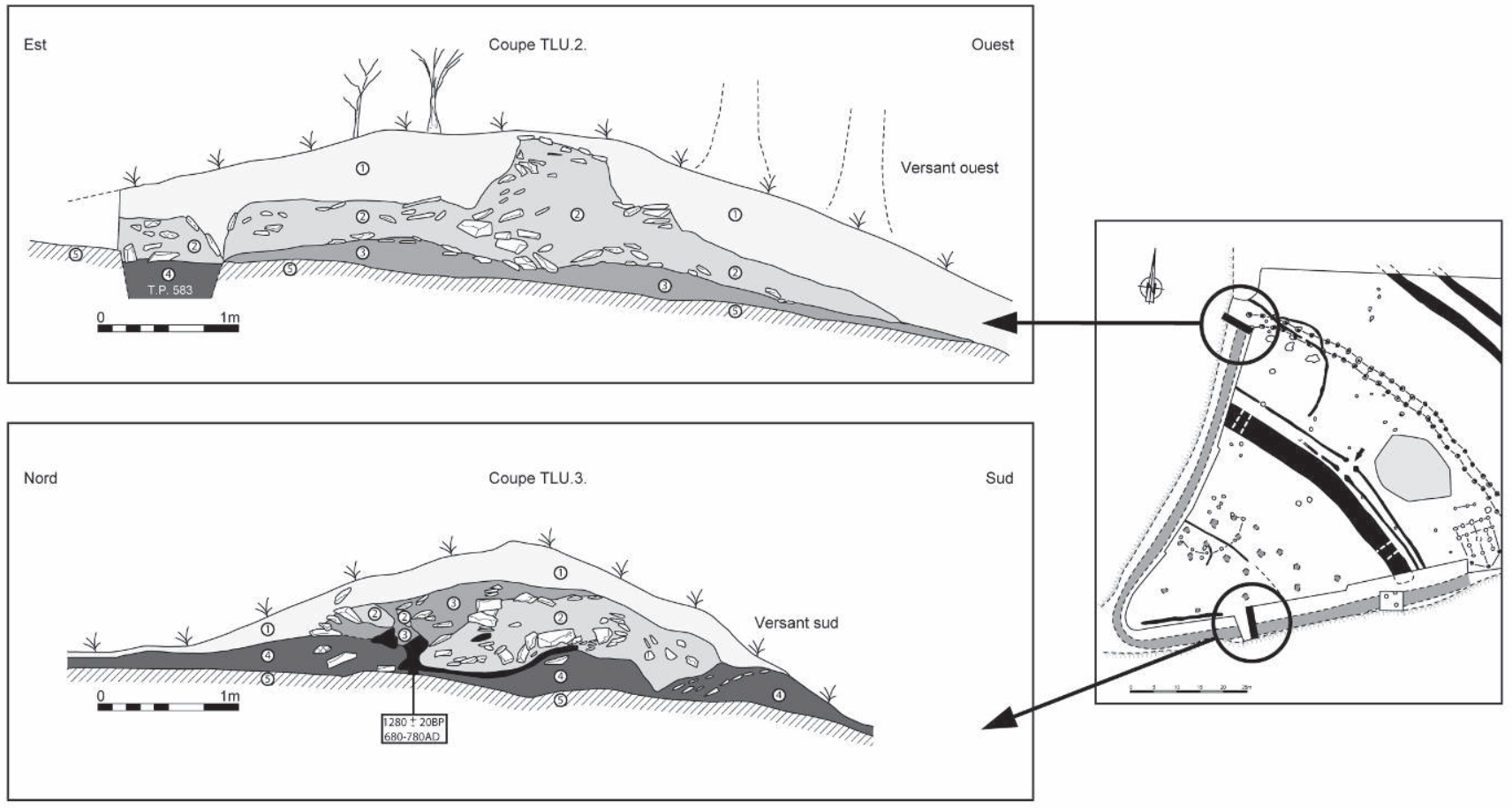

D.A.O L.Quesnel CReAAH du CNRS

Figure 41 : Coupes TLU.2 et TLU.3 du talus 6 b à noyau rubéfié. 1, humus léger et poudreux gris clair avec gravier de schiste; 2 , terre pulvérulente orangé clair avec de nombreuses plaquettes de schiste fortement chauffées, parfois fondues (possible reste de parement sommaire en versant ouest); 3 et 4, terre brun foncé avec petites plaquettes de schiste (sol piégé sous le talus); 5 , substrat de schiste briovérien.

Figure 41: Sections TLU.2 and TLU.3 of bank 66 with reddened core. 1, light and powdery humus, light grey with schist gravel; 2, light orange powdery earth with many schist fragments, heavily burnt and sometimes smelted (possible remain of a makeshift facing on West slope); 3 \& 4, dark brown earth with small flakes of schist (soil under the bank); 5, Brioverian schist substratum.

\section{D. Un bâtiment à foyer central (6c) dans l'angle sud-est de l'enceinte}

Tout près de l'extrémité sud-est de la double palissade 6a et en bordure de la rupture de pente sud, dix-sept trous de poteau (TP.453 à 467, 620 et 621) définissent le plan d’un bâtiment de $10 \mathrm{~m}$ de long et $8 \mathrm{~m}$ de large, orienté nord-sud (fig. 34 et 45). Les dix poteaux périphériques dessinent un trapèze avec pignon nord droit ( $8 \mathrm{~m}$ de large) et pignon sud en $\mathrm{V}$ (7 $\mathrm{m}$ de large), traduisant une abside à cette extrémité. L'espace utile interne atteint ainsi une surface minimale de $75 \mathrm{~m}^{2}$. Les sept poteaux internes délimitent un carré de 


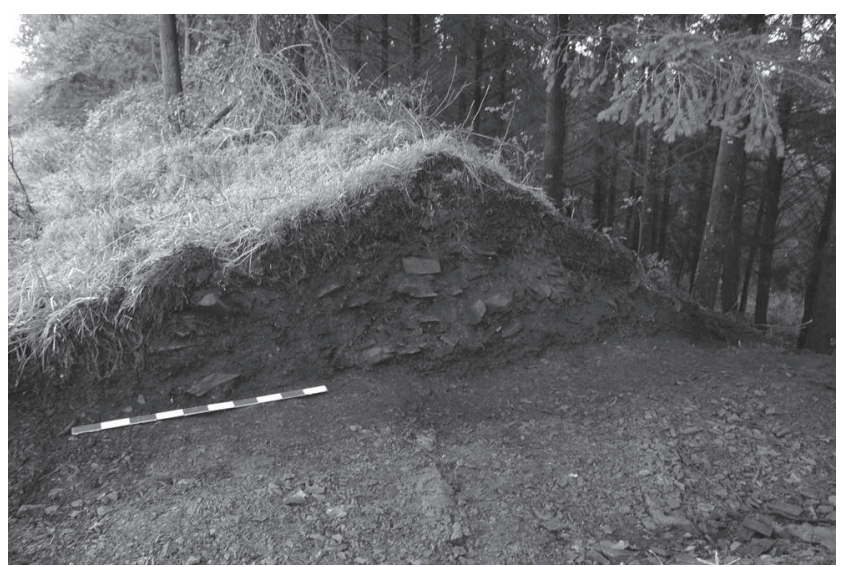

Figure 42 : la coupe TLU.3 du talus bordant le promontoire au sud et daté au haut Moyen Âge par le radiocarbone et la dendrochronologie.

Figure 42: TLU.3 section of the bank on the promontory edge, dated to the early Mediaeval period by radiocarbon and dendrochronology.

$5 \mathrm{~m}$ de côté. Ces fondations sont puissantes pour la taille du bâtiment, et de dimensions régulières. Cependant, les éléments périphériques sont plus légers avec en moyenne un diamètre et une profondeur de $0,55 \mathrm{~m}$ et la quasi-absence de calages; la trace de poteaux de $0,20 \mathrm{~m}$ de diamètre est cependant visible dans cinq d'entre eux (TP.453, 454, 467, $620,621)$. En revanche, les sept poteaux internes sont ancrés à $0,65 \mathrm{~m}$ de profondeur moyenne et les trous contiennent systématiquement de gros blocs de calage, essentiellement en schiste, plus rarement en quartz et, pour un cas, un fragment de meule en granite. La forte obliquité des parois, parfois verticales, assurant un bon calage pour de faibles portées ( $2 \mathrm{~m}$ entre axes), cela renforce l'impression d'une structure centrale très haute à étage. Ces fondations internes ne présentent aucune trace de poteau mais des actions de chauffe (terre cendreuse, blocs et parois rubéfiés...); les calages paraissent perturbés, à la suite possible de l'effondrement de l'édifice.

Au centre du bâtiment, le substrat est nettement rubéfié sur une zone circulaire de $0,90 \mathrm{~m}$ de diamètre, emplacement probable d'un foyer central excavé dans le sol.

À trois mètres et parallèlement au pignon nord, un alignement de trois poteaux plus légers (TP.452, 595, 596; diamètres 0,30 à $0,45 \mathrm{~m}$, profondeurs $0,25,0,50$ et $0,15 \mathrm{~m}$ ) peut être associé au bâtiment (auvent, avant-cour?), mais également à l'écartement plus important des poteaux voisins de la double palissade (on note $3 \mathrm{~m}$ entre les paires TP.446-449 et 447-448 au lieu des $2 \mathrm{~m}$ habituels - voir supra, $\$$ IX A), en lien avec une éventuelle entrée dans l'enceinte et délimitant ainsi un couloir d'accès.
Deux zones cendreuses, de $1 \mathrm{~m}$ de diamètre moyen et contenant quelques galets de quartz éclatés par la chaleur, sont à signaler dans ce secteur, l'une à l'angle nord-est du bâtiment, la seconde hors de l'enceinte près du poteau 468 . En l'absence d'élément de datation, leur fonction reste indéterminée : structures de combustion externes contemporaines du bâtiment, éléments diachroniques, lien avec les zones cendreuses de l'extrémité ouest (voir supra, $\$$ IX A)?

Malgré un tamisage des comblements, les fondations de l'édifice n'ont pas livré de mobilier, à l'exception de deux fragments de meule en granite, d'un petit fragment de fer (clou) et de quelques échantillons de clayonnage très morcelés.

Deux datations radiocarbone ont été obtenues sur charbon de bois : TP.455: GrN-30620: $1235 \pm 30$ BP, soit 680-890 apr. J.-C. en âge calibré et TP.461 : GrN-30619 : $1200 \pm 50$ BP, soit 680-980 apr. J.-C. en âge calibré. Elles corroborent celles obtenues sur la double palissade 6a et le talus $6 \mathrm{~b}$ pour une attribution de cet ensemble très structuré au haut Moyen Âge (fin vII ${ }^{\mathrm{e}}$-VIII ${ }^{\mathrm{e}}$ siècles).

Par ailleurs, le plan général fait apparaître un parallélisme et une équidistance remarquables entre les fossés centraux $4 \mathrm{a}$ et $4 \mathrm{~b}$ et la double palissade $6 \mathrm{a}$, ce qui pourrait indiquer un ensemble du haut Moyen Âge plus conséquent : la sinuosité des tracés est comparable tandis que la distance de $20 \mathrm{~m}$ entre les structures est constante; cette similitude est renforcée par le plan et les dimensions très proches des bâtiments $4 c$ et $6 c$.

À l'instar des périodes précédentes, plusieurs échantillons de charbons de bois des différentes structures du haut Moyen Âge ont été analysés. Au cortège déjà rencontré - chêne dominant, noisetier et fabacées (genêt/ajonc) - s'ajoutent trois taxons nouveaux : le frêne, le saule et le hêtre. D'implantation tardive dans le Massif armoricain, ce dernier semble être exploité en concurrence avec le chêne à partir du haut Moyen Âge. L'étude des cernes du chêne révèle une sélection des bois en fonction du type de structure à édifier et une relation étroite entre l'homme et la forêt. Par ailleurs, la présence du frêne peut être liée aux pratiques agropastorales (voir infra, $\$$ XII B4).

\section{E. La zone centrale du site (J.-Y. T.)}

\section{IXE 1. Les trois fossés $4 a-4 c$}

La zone centrale du promontoire, marquée par un léger rehaussement du relief découlant d'une meilleure résistance du socle rocheux à l'érosion, est relativement pauvre en vestiges (fig. 4 et 46 à 49). Un premier fossé (4a), long de $60 \mathrm{~m}$, barre la partie centrale mais s'interrompt bien avant les flancs de l'éperon (fig. 46). À une quinzaine de mètres vers le sud, deux fossés parallèles ( $4 \mathrm{~b}$ et $4 \mathrm{c}$ ) assurent cette fois 


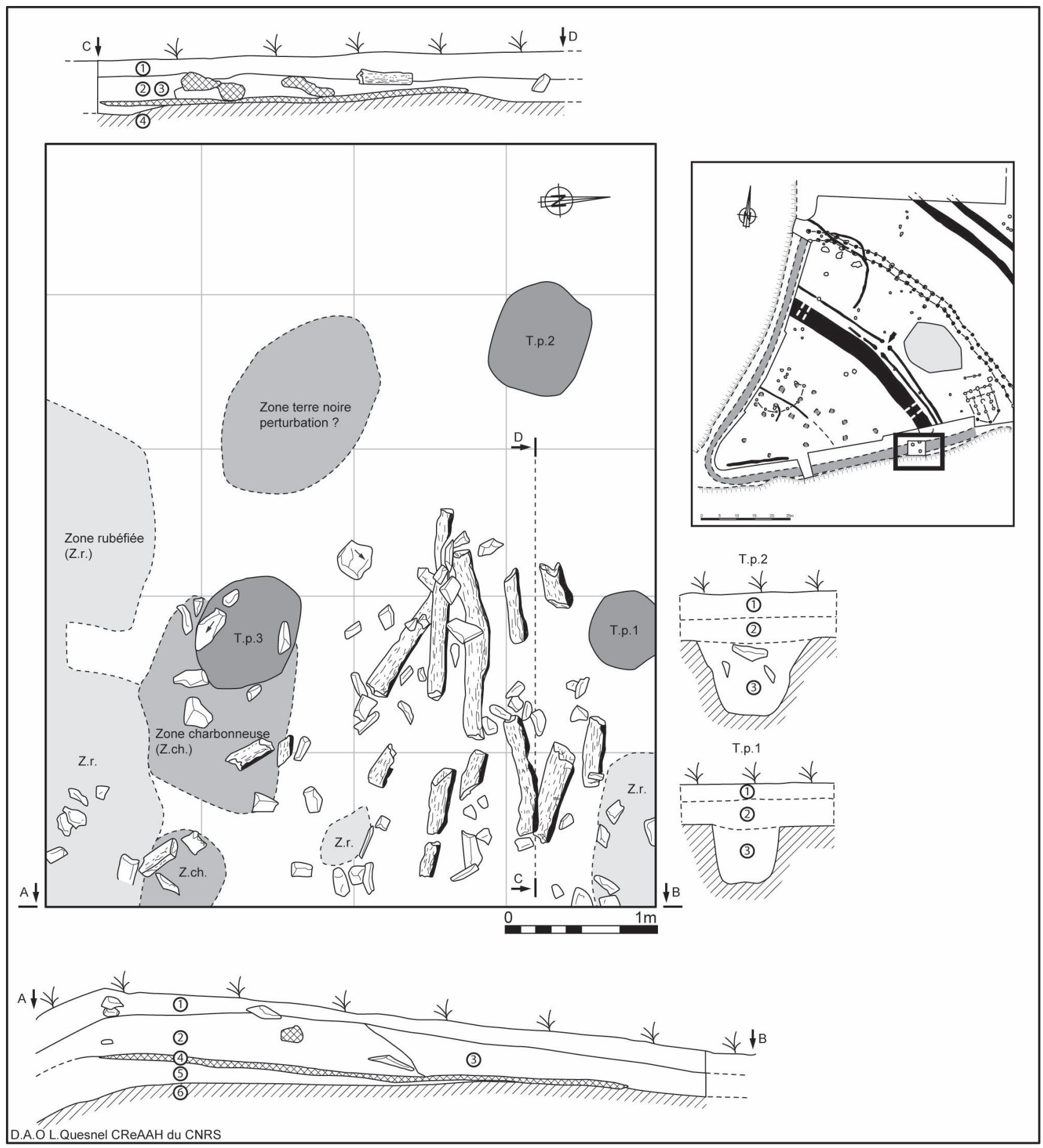

Figure 43 : Secteur sud-est du talus du haut Moyen Âge : pièces de bois calcinées, zones rubéfiées (zr), zones charbonneuses (z.ch.) et trous de poteau (tp) mis au jour sous le talus dans le sondage TLU.4. Coupe transversale AB : 1, terre végétale pulvérulente grise; 2 , terre et plaquettes de schiste rubéfiées orange clair; 3 , terre grasse noire charbonneuse; 4, lit de charbon de bois; 5 , terre végétale brun clair (paléosol piégé); 6 , substrat de schiste briovérien.

Figure 43: South-east sector of the early Mediaeval bank: burnt pieces of wood, reddened zones (zr), charcoal zones (z.ch.) and post-holes (tp) discovered under the bank in TLU.4 sondage. A-B section: 1, grey powdery soil; 2, earth and reddened schist fragments; 3, sticky carboniferous black earth; 4, bed of charcoal; 5, light brown buried paleosoil; 6, brioverian schist substratum. 

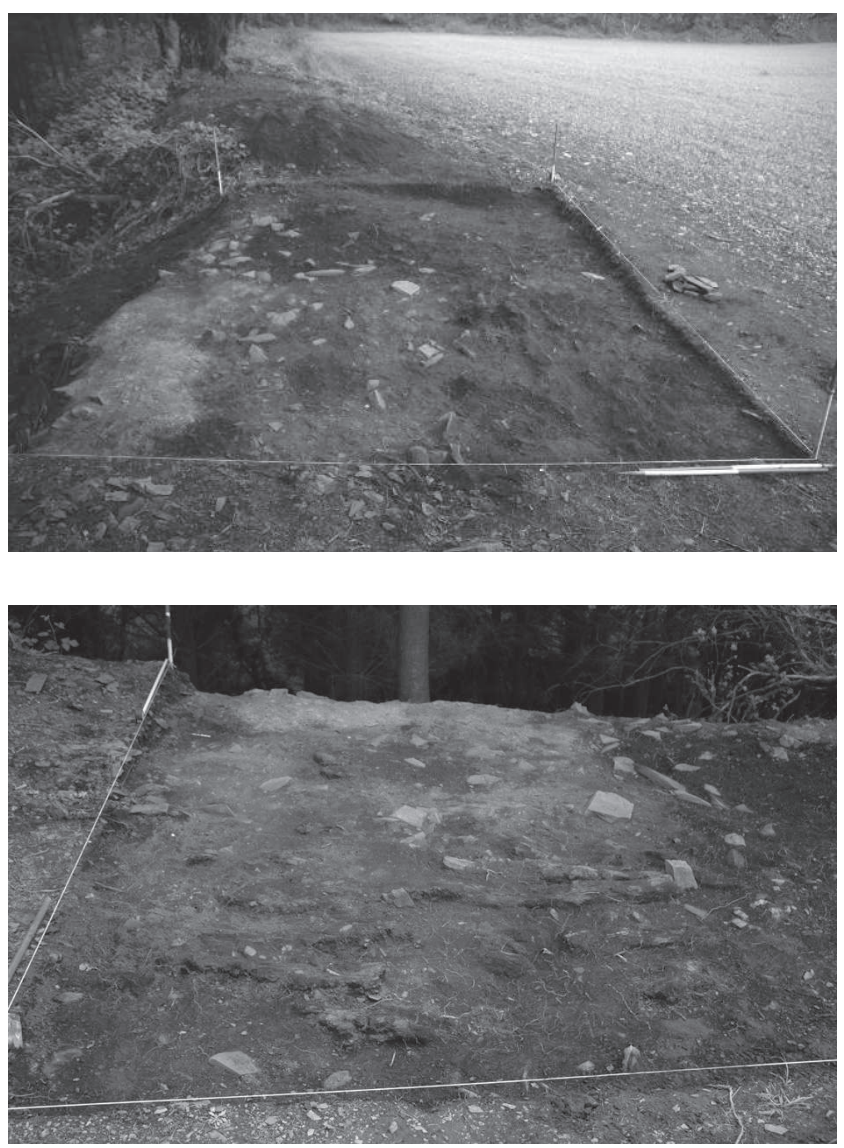

Figure 44 : Vue de l'est (en haut) et du nord (en bas) du sondage TLU.4 dans le talus du haut Moyen Âge, secteur sud-est. Le noyau schisteux rubéfié est visible en bordure sud externe à l'enceinte; les pièces de bois calcinées sont disposées à plat en bordure nord interne (sondage réalisé en mai 2007 après rebouchage des fouilles et remise en culture).

Figure 44: View from the east (above) and from the north (below) of the early Mediaeval sondage TLU.4. The reddened schist core can be seen on the external edge of the enclosure; the charred pieces of wood lay horizontally on the north internal edge.

un barrage complet sur une longueur de $115 \mathrm{~m}$, tout en laissant un passage entre leurs extrémités et les versants sud et ouest; leur écartement moyen de 3 à 4 m laisse supposer la présence d'un talus intermédiaire. Les dimensions moyennes de ces trois fossés sont de 1,50 à $2,50 \mathrm{~m}$ de large et 0,50 à $0,80 \mathrm{~m}$ de profondeur. Leur profil est en $\mathrm{V}$, plus ou moins accentué suivant les secteurs. Le comblement terreux brun sombre présente une superposition de deux ou trois couches disposées en berceau qui ne se distinguent que par une plus grande concentration de plaquettes de schiste en fond de fossé. La réalisation de huit sondages n'a livré aucun élément de datation, à l'exception d'un gros éclat de silex cortical et d'un fragment de fer, ces deux pièces étant mises au jour en sommet de comblement. On remarquera le parallélisme

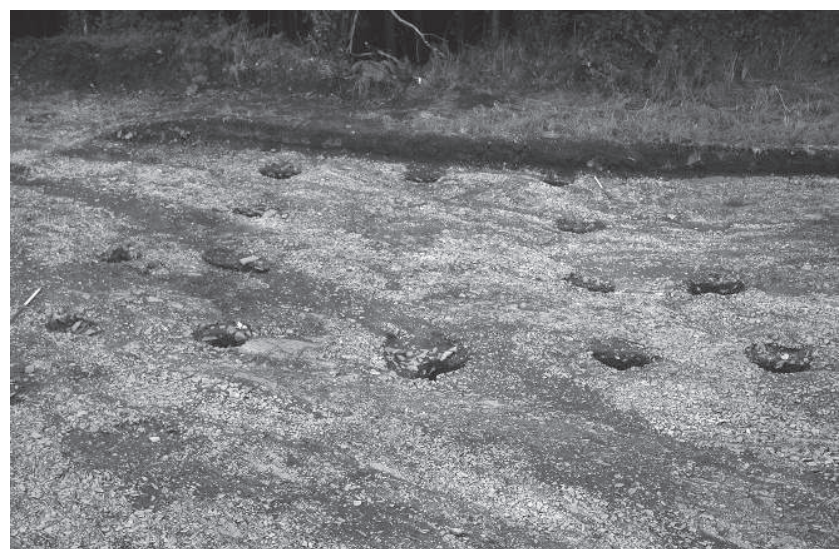

Figure 45 : Vue du nord de la maison 6c datée au haut Moyen Âge dans l'angle sud-est de l'enceinte. Une trace rubéfiée circulaire au centre révèle la présence d'un foyer excavé. À ce stade de la fouille, le pignon sud en abside n'est pas encore dégagé sous la berme en arrière plan.

Figure 45: View from the north of early Mediaeval house $6 c$ on the south-east angle of the enclosure. A reddened circular trace in the centre reveals an dished hearth. At this stage, the south apse gable is not yet cleared under the background berm.

dans la sinuosité de ces trois éléments dont le tracé marque toutefois une rupture très nette avec les ensembles attribués au Bronze et au premier Fer décrits précédemment et situés au nord (voir supra, $\$$ VII et VIII).

\section{IXE 2. Le bâtiment central $4 d$}

Près de l'extrémité sud-est du premier fossé, un ensemble de sept trous de poteau (TP.410 à 416) dessine le plan d'un bâtiment (4d), orienté nord-est - sud-ouest (fig. 48 et 49). Légèrement trapézoïdal (largeur 5,50 à 6 m), l'édifice se termine par un pignon nord-est en abside pour atteindre une longueur de $10 \mathrm{~m}$. L'espace utile interne est ainsi de $60 \mathrm{~m}^{2}$ environ. Les trous de poteau ont une morphologie homogène : 0,55 à $0,60 \mathrm{~m}$ de diamètre, $0,25 \mathrm{à} 0,30 \mathrm{~m}$ de profondeur, rares éléments de calage, traces de poteaux de $0,20 \mathrm{~m}$ de diamètre. Le poteau de la pointe nord-est (416) semble doublé et son trou a piégé plusieurs tessons d'une céramique très grossière et recuite (creuset?). L'hypothèse d'un atelier destiné au travail du fer peut être émise grâce à la présence de fragments de scories de fer dans les trous 411 et 413 et à celle, au centre de l'édifice, de quatre trous (588-591) très peu profonds $(5 \mathrm{~cm})$, emplacement possible d'une structure de travail. Sur le flanc ouest, une rigole peu marquée dans le substrat ( $5 \mathrm{~cm}$ maximum) peut être interprétée comme la base de la paroi latérale. Une structure de combustion toute proche et contenant des blocs de quartz brûlés rappelle la

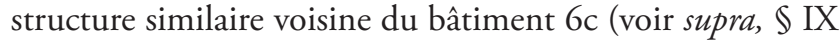
D). Par sa morphologie et ses dimensions, l'édifice $4 \mathrm{~d}$ est 


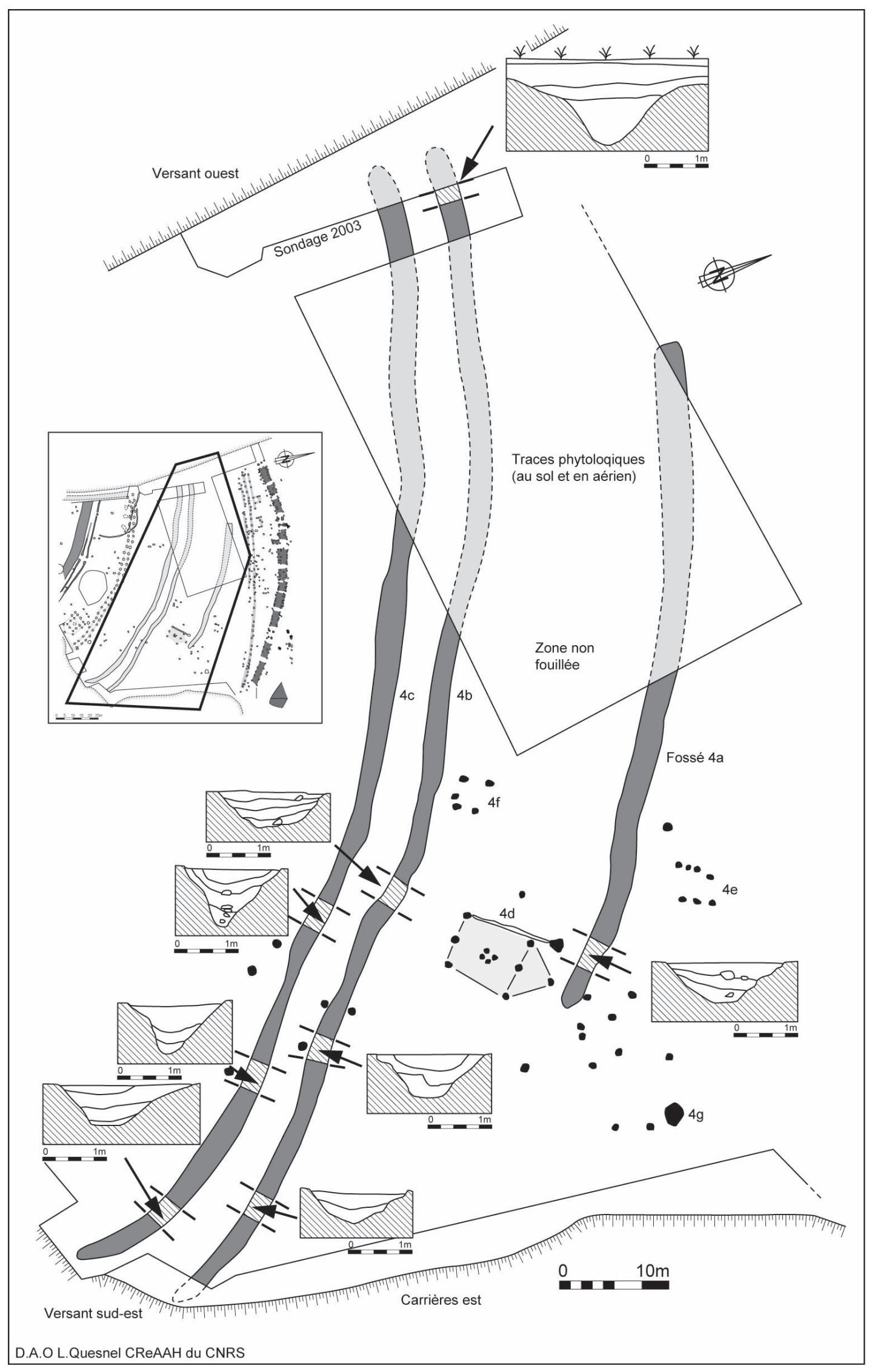

Figure 46 : Zone centrale du promontoire : fossés parallèles 4a, 4b, $4 \mathrm{c}$ et coupes, bâtiment à abside $4 \mathrm{~d}$, ensembles de trous de poteau $4 \mathrm{e}$ et $4 \mathrm{f}$, structure de combustion $4 \mathrm{~g}$.

Figure 46: Central zone of the promontory: parallel ditches $4 a, 4 b, 4 c$ and sections, apsed building $4 d$, post-holes wholes $4 \mathrm{e} \& 4 \mathrm{f}$, hearth $4 \mathrm{~g}$. 

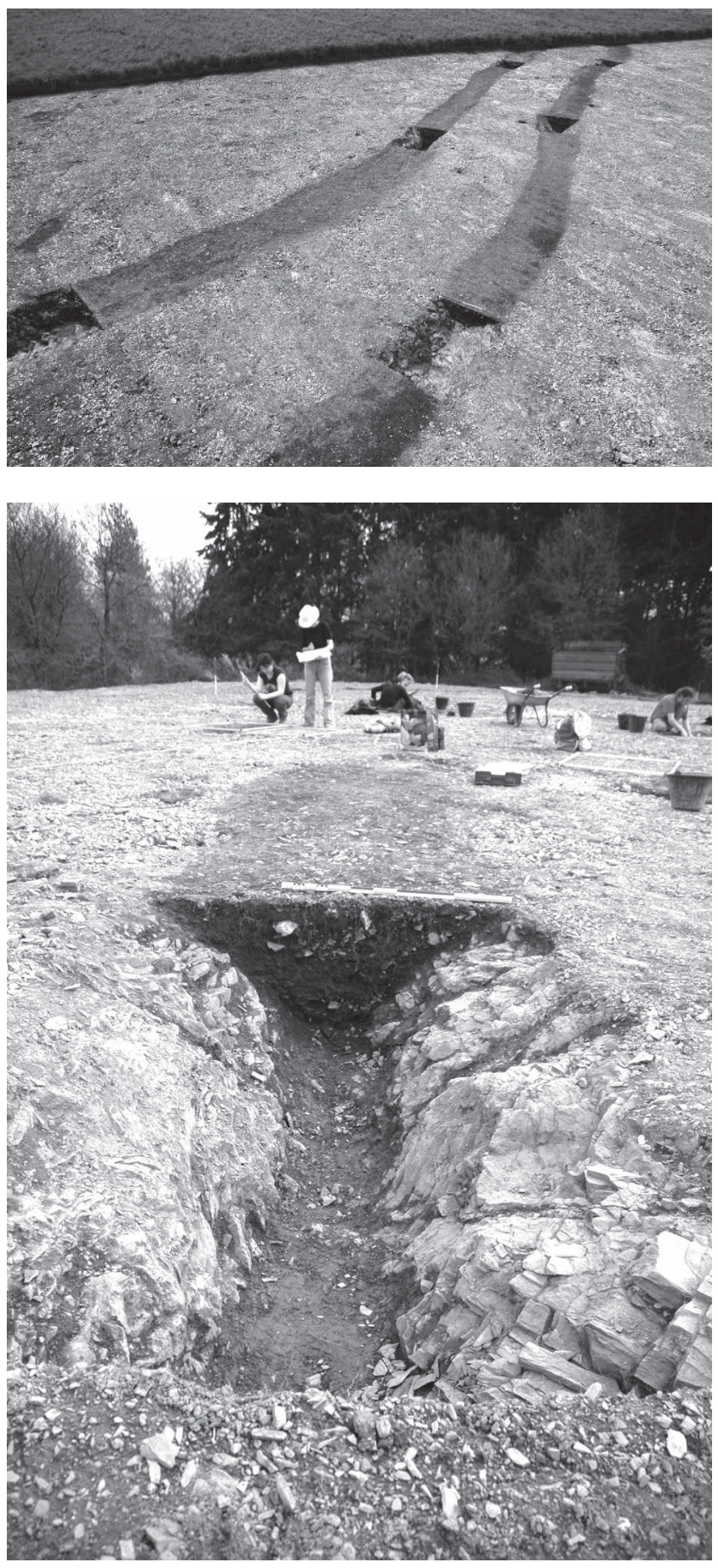

Figure 47 : En haut, vue vers le nord-ouest des fossés parallèles $4 \mathrm{~b}$ et $4 \mathrm{c}$ en avril 2006. En-bas, vue de détail vers le sud-est d'une coupe du fossé $4 \mathrm{~b}$.

Figure 47: On top, view from the north-west of the parallel ditches $4 b$ and $4 c$. Under, detail view towards the south-east of a section in ditch $4 b$.

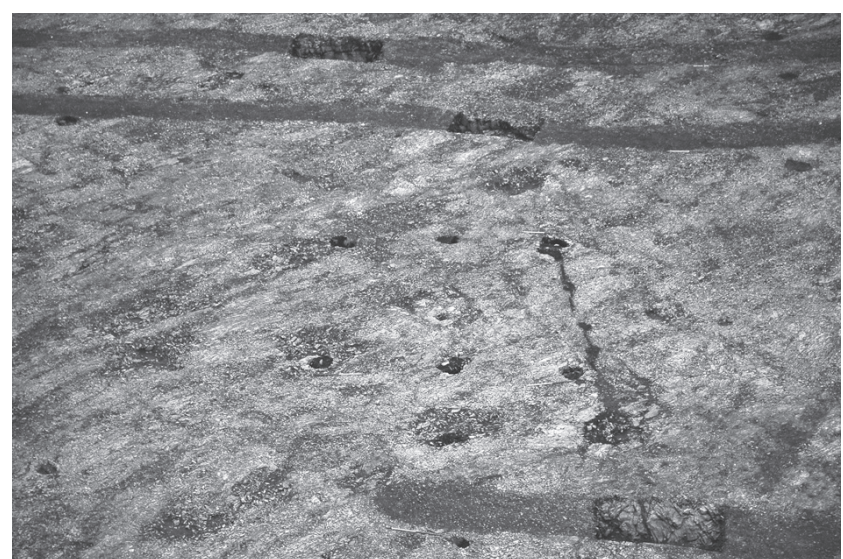

Figure 48 : Vue vers le sud-ouest du bâtiment central 4d entre l'extrémité du fossé $4 \mathrm{a}$ et les fossés $4 \mathrm{~b}-4 \mathrm{c}$.

Figure 48: View towards the south-west of central building $4 d$ between the end of ditch $4 a$ and ditches $4 b-4 c$.

comparable à $6 c$, avec toutefois des fondations nettement plus légères pour une construction plus modeste.

Ces structures centrales (fossés $4 \mathrm{a}$, $4 \mathrm{~b}$ et $4 \mathrm{c}$, bâtiment 4d) ne peuvent être datés directement par leur mobilier. Cependant, le tracé des fossés révèle une similitude avec la palissade de bois du haut Moyen Âge. La sinuosité et le parallélisme de 4a, 4b, 4c se retrouvent dans la palissade 6a et un espace régulier d'une vingtaine de mètres sépare ces barrages successifs (cf. infra, fig. 63-3). Il en va de même pour la distance entre la palissade $6 \mathrm{a}$ et le fossé $\mathrm{f} 5$, creusé à l'âge du Fer mais certainement encore visible au viI ${ }^{\mathrm{e}}$ siècle après J.-C. Par ailleurs, les plans à abside des bâtiments $4 \mathrm{~d}$ et $6 \mathrm{c}$ sont très proches. Ainsi, une emprise du site du haut Moyen Âge à partir du fossé $4 a$ jusqu'à la pointe de l'éperon peut être envisagée.

\section{IXE 3. Quelques éléments isolés complémentaires}

Une trentaine de petits trous de poteau complémentaires a été mise au jour dans ce secteur central mais leur disposition aléatoire se prête peu à l'interprétation (fig. 46). En bordure et de part et d'autre des fossés $4 \mathrm{~b}$ et $4 \mathrm{c}$, quelquesuns pourraient signaler la présence d'ouvrages de franchissement. Deux ensembles mieux regroupés, $4 \mathrm{e}$ et $4 \mathrm{f}$, pourraient signaler de petits bâtiments de type grenier et l'aspect des micro-tessons très érodés qui en proviennent va dans le sens d'une attribution à la Protohistoire par comparaison avec le matériel céramique mis au jour sur la partie nord du site. 


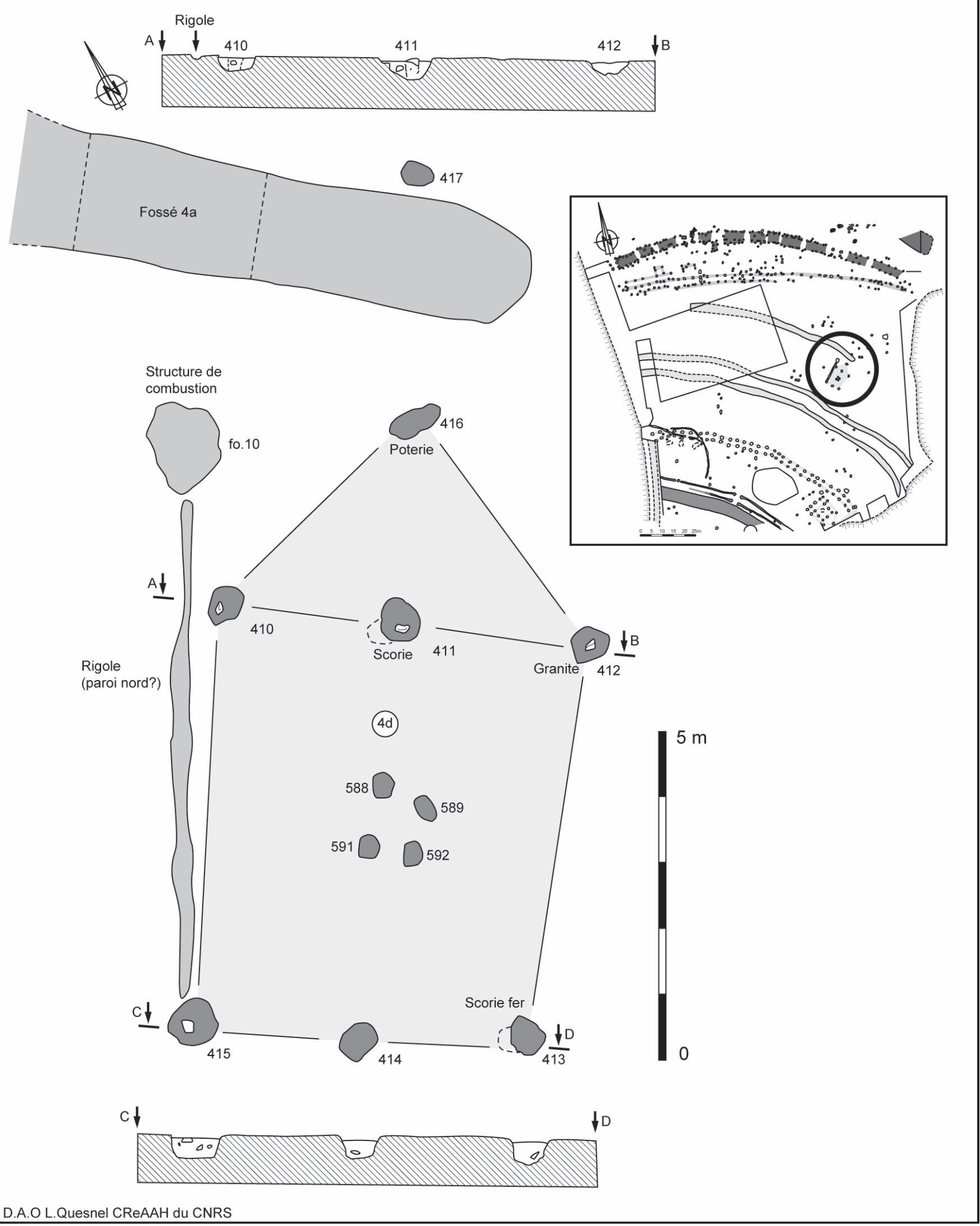

Figure 49 : Zone centrale du promontoire : le bâtiment à abside $4 \mathrm{~d}$.

Figure 49: The apsed building $4 d$ in the centre of the promontory. 


\section{F. DEUX PETITS ENCLOS ADOSSÉS AU FLANC SUD-OUEST DU PROMONTOIRE (ENSEMBLE 7) (J.-Y. T.)}

\section{F 1. L'enclos $7 a$ et la double palissade $7 b-7 c$}

En bordure sud-ouest du promontoire, une structure curvilinéaire (7a) se surimpose à l'extrémité nord-ouest de la double palissade 6a; elle est composée de tronçons (fo.29 à fo.33) d'une tranchée étroite ( 0,15 à $0,30 \mathrm{~m}$ de large) et très peu profonde $(0,05$ à $0,15 \mathrm{~m})$ (fig. $4,35,36,37)$. Le comblement se compose d'une terre très sombre et la présence de poteaux plus importants par endroits est marquée par un élargissement circulaire de la tranchée. Le recoupement avec plusieurs fosses de calage (TP.569, TP.571 et TP.579) montre de façon incontestable sa postériorité par rapport à la double palissade 6a du haut Moyen Âge.

Cette palissade curviligne $7 \mathrm{a}$ s'adosse au sud à la tranchée du même type fo. 28 qui appartient à l'ensemble $7 \mathrm{~b}-7 \mathrm{c}$ (fig. 37). Ce dernier est composé de tranchées similaires en quatre tronçons rectilignes (fo.25 à fo.28), parallèles au grand fossé 5 . Une interruption de $2 \mathrm{~m}$ de large matérialise, à équidistance des flancs du promontoire, une entrée monumentale de $2 \mathrm{~m}$ sur $2,50 \mathrm{~m}$ entre quatre trous de poteau de fortes dimensions (TP.493 à 496 : diamètres 0,80 à $1 \mathrm{~m}$, profondeurs 0,20 à $0,50 \mathrm{~m}$ ) (fig. 29 et 30 ). Un échantillon de charbon de TP.494 donne une datation du second âge du Fer (GrN-30623: $2240 \pm 30$ BP, soit 400-200 av. J.-C.). Cette datation et le parallélisme avec le fossé 5 feraient pencher pour le rattachement des palissades 7b-7c à la Protohistoire; toutefois, la présence de céramique du Moyen Âge, un fragment de lèvre plate notamment (cf. infra, fig. 51, n ${ }^{\circ}$ ), nous impose de revoir la datation et prendre en considération deux phénomènes, d'une part, le piégeage de mobilier résiduel antérieur lors du creusement des tranchées, d'autre part, la pérennité du tracé du fossé 5 protohistorique orientant le tracé des palissades médiévales, comme déjà évoqué auparavant.

Les tranchées des ensembles $7 \mathrm{a}$ et $7 \mathrm{~b}$ délimitent ainsi un enclos de forme subrectangulaire de $350 \mathrm{~m}^{2}$ environ, établi en bordure ouest du promontoire. On y accède par deux entrées, l'une au sud-est matérialisée par les TP.563 et 564, la seconde au nord entre fo. 29 et fo.30, bordée également de petits trous de poteau et protégée par la palissade fo.33 (fig. 37). Un trou de poteau important (TP.586) marque le centre de l'espace ainsi défini. La relative légèreté des structures et la configuration de l'enclos, avec l'entrée nord en chicane en particulier, étayent l'hypothèse de structures destinées à la gestion d'un cheptel.

\section{F 2. L'ensemble 7d}

À une vingtaine de mètres de la pointe de l'éperon, un ensemble d'une quinzaine de trous de poteau (TP.603 à 617) sont regroupés en bordure de la pente ouest (fig. 39). Plusieurs éléments amènent à les associer : dimensions similaires $(0,45$ à $0,60 \mathrm{~m}$ de diamètre, 0,20 à $0,35 \mathrm{~m}$ de profondeur, trace de poteau de $0,20 \mathrm{~m}$ de diamètre) et même comblement brun gris. La présence de mobilier permet de les dater du Moyen Âge : deux fragments de fer dont une lame de couteau en TP.615 (cf. infra, fig. 51, n 12), une lèvre de vase à rebord horizontal caractéristique des $\mathrm{X}^{\mathrm{e}}$ $\mathrm{XII}^{\mathrm{e}}$ siècles en TP.609 ( $\left.\mathrm{n}^{\circ} 11\right)$. Une structure semi-circulaire de 10-12 $\mathrm{m}$ de diamètre peut ainsi être définie, délimitant une emprise d'environ 250 à $300 \mathrm{~m}^{2}$. Bien que de nature différente de l'enclos 7a, une utilisation comme enclos à bestiaux peut être également évoquée pour cette structure.

\section{G. Un verger du $X^{\mathrm{e}}$ siècle en pointe de promontoire}

Lors du décapage des terres végétales en pointe sud-ouest de l'éperon, une vingtaine de creusements a immédiatement retenu l'attention par leur net contraste sur le schiste. Il s'agit de fosses généralement de plan carré, plus rarement rectangulaire, d'environ $1 \mathrm{~m}$ de côté. Leur profondeur dans le schiste ne dépasse pas $0,20 \mathrm{~m}$ et elles sont comblées de terre végétale sombre. La présence de quelques fragments de faïence, de verre de bouteille et de clous de fer a permis de dater rapidement ces fosses du Xx ${ }^{\mathrm{e}}$ siècle et de les interpréter comme fosses de plantation d'un verger, ce qui a été confirmé par quelques habitants âgés du hameau de La Rochette. Anecdotiques pour l'histoire du site, ces fosses sont toutefois intéressantes par leur répartition sur trois lignes en arcs de cercle parallèles au fossé 5 dont l'impact était partiellement conservé jusqu'au remembrement des années 1960 (fig. 39). Par ailleurs, un espace vide de fosses sépare le verger du fossé, ce qui pourrait être interprété comme la présence d'un résidu de rempart longeant le bord interne du fossé 5, l'épaisseur de terre étant ainsi suffisante pour que ces excavations n'atteignent pas le substrat. Une seule fosse est présente à proximité du fossé 5 en partie centrale : est-ce l'emplacement d'un passage central dans le rempart en vis à vis de la porte monumentale délimitée par les TP.493 à 496?

\section{H. Le mobilier archéologique mis au jour sur l'aire centrale et sud du promontoire (J.-Y. T.)}

Sur cette zone fortement marquée par l'implantation médiévale, le mobilier céramique comprend 86 éléments, pour un poids de 2080 g. Essentiellement représenté par 
des tessons isolés, cet ensemble comprend toutefois deux lots plus conséquents constitués de plusieurs tessons d'un même vase et totalisant $1490 \mathrm{~g}$, soit plus de $70 \%$ du poids de l'ensemble. Ceci met en évidence l'extrême indigence du corpus sur l'ensemble de la partie sud du site. Ce matériel a été majoritairement (à $75 \%$ ) mis au jour dans le comblement des trous de poteau et tranchées de fondation des enclos, plus rarement à l'interface entre la terre végétale et le substrat lors du nettoyage superficiel (fig. 50). Les caractéristiques générales de la céramique se distinguent sensiblement

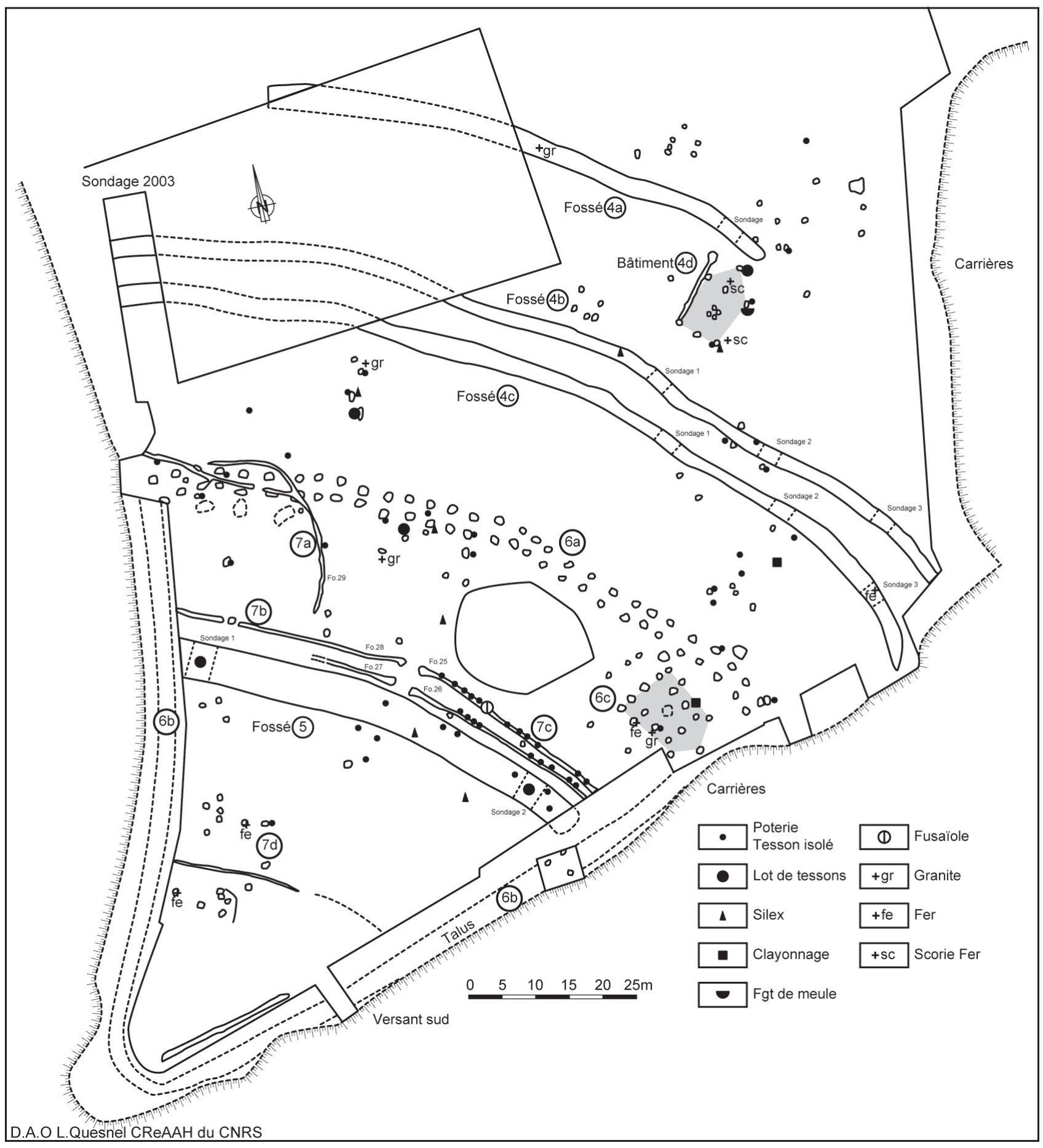

Figure 50 : Répartition du mobilier sur la zone centrale et la pointe sud du promontoire.

Figure 50: Artefacts distribution on the central zone and the south point of promontory. 
de celles du secteur nord, en raison notamment de la qualité des pâtes. Le morcellement et le taux d'usure sont moins importants avec $47 \%$ des tessons de taille inférieure à $4 \mathrm{~cm}^{2}$ et $61 \%$ aux contours érodés. Les teintes claires, beige et brun orangé, restent majoritaires avec $67 \%$ de l'ensemble. Les pâtes sont de meilleure qualité avec un dégraissant fin et calibré pour $60 \%$ des cas, une bonne cuisson ( $57 \%$ ) et un lissage des surfaces plus fréquent (57\%). Les remontages sont impossibles et les décors absents; les éléments de formes se limitent à huit fragments de lèvres et un fond plat.

Par la nature des pâtes et la morphologie, certaines pièces peuvent se rattacher à l'occupation de l'âge du Bronze implantée au nord du site. Mis au jour dans une anfractuosité du substrat entre la double palissade $6 a$ et le fossé 5 , un lot de tessons provient d'un vase globulaire à profil en $S$, paroi épaisse de 10-12 mm (fig. 51, $\mathrm{n}^{\circ} 1$ ). La pâte est fragilisée par un dégraissant de quartz roulé très dense et le montage aux colombins est visible par l'aspect bosselé et grossier de la surface. Trouvé sur le schiste également, près $\mathrm{du}$ bord sud du fossé 5 , un bord de vase épais (12$13 \mathrm{~mm}$ ), à profil hémisphérique, présente une lèvre aplatie et lissée (fig. $51, \mathrm{n}^{\circ} 2$ ); la pâte, dégraissée de grains de quartz moyens, est bien cuite.
Figure 51: Mobilier de la pointe sud du promontoire : $1,2,4,5$, céramique de l'âge du Bronze; 3 , céramique de l'âge du Fer (TP.489); 6, lèvre à rebord, pâte gris-noir, haut Moyen Âge (TP.600); 7, fusaïole en céramique (tranchée fo. 25) ; 8, fond plat à pâte orange-clair, Moyen Âge (tranchée fo.25); 9, grattoir en silex noir (TP.490) ; 10, lèvre aplatie, pâte orange-clair, Moyen Âge (fossé 5); 11 , lèvre à rebord, pâte beige-clair, $\mathrm{X}^{\mathrm{e}}-\mathrm{XII}^{\mathrm{e}}$ siècles (TP.609); 12, : lame de fer (TP.615).

Figure 51: Material from South point of promontory: 1, 2, 4, 5, Bronze Age ceramics; 3, Iron Age ceramic (TP.489); 6, rim, dark grey ware, early Mediaeval (TP.600); 7 , ceramic spindle-whorl (trench fo.25); 8, flat bottom, light orange ware, Mediaeval (trench fo. 25); 9, black flint scraper (TP.490) 10, flattenned rim, light orange ware, Middle Ages (ditch 5); 11 , rim, light beige ware, $10^{\text {th }}$ $12^{\text {th }}$ centuries (TP.609); 12, iron blade (TP.615).

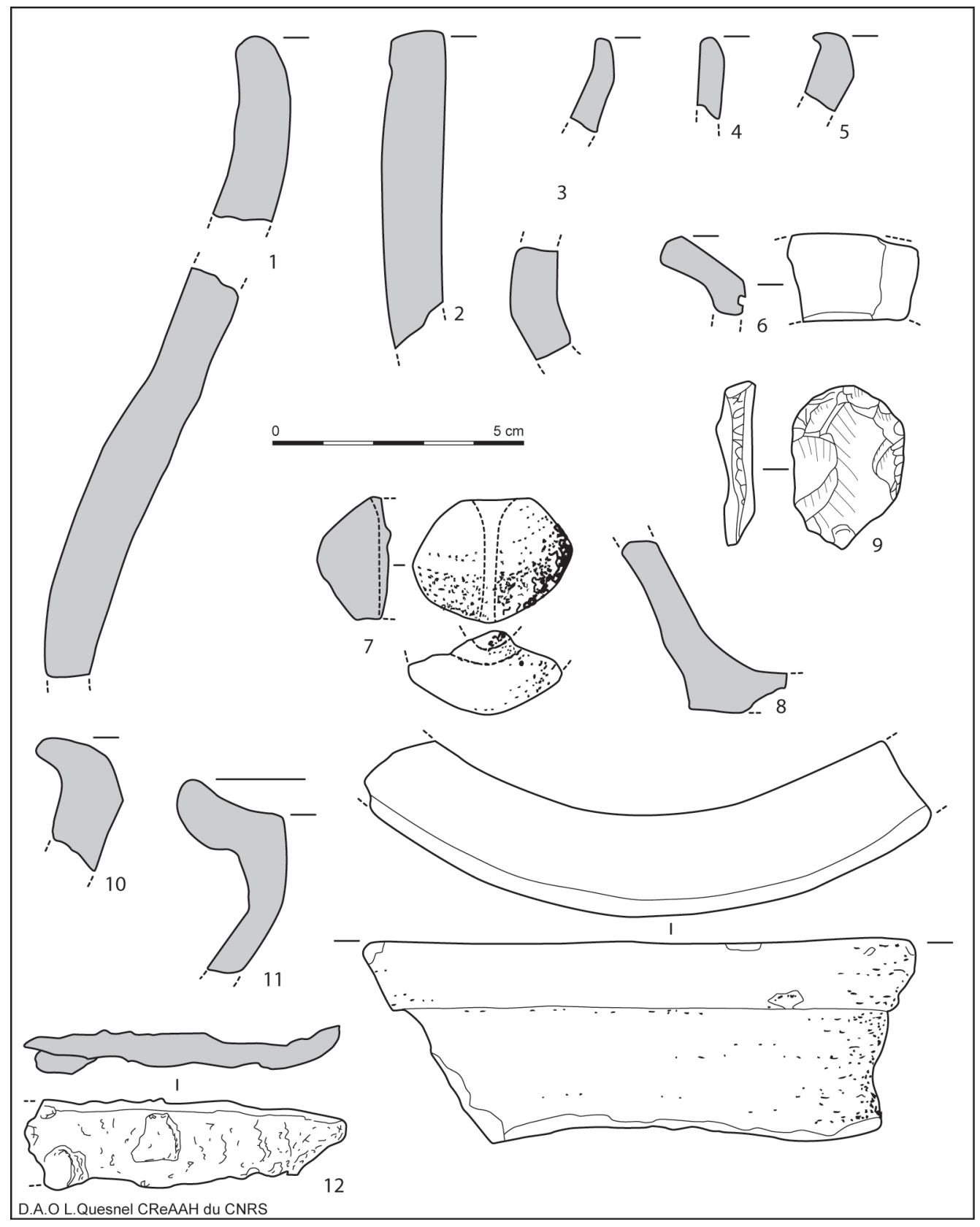


Quelques rares éléments confirment l'occupation du Moyen Âge. Un fragment de lèvre à méplat éversé légèrement concave provient d'un petit trou de poteau (TP.600) en bordure du fossé $5\left(\mathrm{n}^{\circ} 6\right)$; sa morphologie et sa pâte noire lissée en font un élément attribuable à l'époque carolingienne et qui corrobore la série de datations de la fin $\mathrm{VII}^{\mathrm{e}}$ au IX ${ }^{e}$ siècles obtenues sur l'enceinte et le bâtiment sud associé. De ce secteur également (tranchée fo.25) provient un fragment de fusaïole biconique en terre cuite rouge foncé de $3 \mathrm{~cm}$ de diamètre $\left(\mathrm{n}^{\circ} 7\right)$.

Quelques éléments attribuables à un Moyen Âge plus avancé sont issus des fondations des enclos semi-circulaires recoupant l'enceinte du haut Moyen Âge. On notera un fragment de fond plat à pâte micacée issu de la tranchée fo. 25 (fig. $51, n^{\circ} 8$ ), un fragment de lèvre à rebord à pâte fine orangé clair ( $\left.\mathrm{n}^{\circ} 10\right)$, un fragment de pot à pâte blanche, lèvre à rebord éversé et face supérieure légèrement concave, caractéristique des $\mathrm{X}^{\mathrm{e}}$-XII ${ }^{\mathrm{e}}$ siècles $\left(\mathrm{n}^{\circ} 11, \mathrm{TP} .609\right)$. Ce dernier vase peut être comparé à la forme $\mathrm{n}^{\circ} 7$ de la typo-chronologie mise en place à partir de la céramique onctueuse dans la région quimpéroise (Villard, 2005, p. 363). Enfin, le trou de poteau 615 du même ensemble a livré un fragment de lame de couteau en fer $\left(\mathrm{n}^{\circ} 12\right)$.

Le matériel lithique taillé n'est attesté que par trois éclats de silex (un noir et deux blonds) et un grattoir sur silex noir opaque dont le front circulaire est obtenu par retouches abruptes (fig. $51, \mathrm{n}^{\circ}$ 9). Notons aussi la présence de deux fragments de scories de fer dans les TP. 411 et 413 du bâtiment central $4 \mathrm{~d}$ et de deux lots de fragments de clayonnage, l'un dans le trou de poteau 457 du bâtiment sud 6c et l'autre, avec empreintes de baguettes de bois, non loin de l'extrémité sud-est du fossé 4c.

\section{I. Éléments de comparaison et contexte historique des occupations médiévales (J.-Y. T.)}

Peu de recherches archéologiques régionales ont abordé la question des sites fortifiés du haut Moyen Âge mais les habitats de cette époque se révèlent de plus en plus nombreux grâce au développement de l'archéologie préventive. Dans son ouvrage de synthèse consacré aux fortifications du haut Moyen Âge en Bretagne, Philippe Guigon (1997) fait le constat qu'avant le $\mathrm{XI}^{\mathrm{e}}$ siècle, rares sont les résidences fortifiées connues n'utilisant pas un relief naturel comme élément défensif et la position topographique de La Rochette ne le contredit pas. Son inventaire des fortifications met en évidence la difficulté de dater ces ouvrages sans entreprendre d'étude approfondie de chaque site, difficulté accentuée par les occupations et transformations multiples dont la plupart ont été l'objet. Deux opérations menées dans les années
1980 ont permis de mieux connaître ces ouvrages du haut Moyen Âge.

L’enceinte circulaire du Camp de Péran à Plédran (Côtesd'Armor) a fait l'objet de fouilles programmées sous la direction de J.-P. Nicolardot entre 1983 et 1990 (Nicolardot et Guigon, 1991) et la datation de la fortification au haut Moyen Âge a été précisée. Cette enceinte ovale d'une surface interne légèrement supérieure à un hectare $(160$ x $147 \mathrm{~m})$ occupe un point haut dans une topographie assez douce dominant la baie de Saint-Brieuc. Sur une occupation antérieure datant de La Tène moyenne et finale, plusieurs datations obtenues par le radiocarbone placent la construction du rempart dans le courant des VIII $^{\mathrm{e}}$ et $\mathrm{IX}^{\mathrm{e}}$ siècles de notre ère avec une destruction au cours du $\mathrm{x}^{\mathrm{e}}$ siècle. La fortification est composée d'un double fossé précédant un rempart de pierres à glacis externe d'argile. La structure interne du rempart a révélé une armature en coffrages de poutres de bois dont la combustion a entraîné la fusion des moellons en roches diverses (quartzites, gneiss, granites et dolérites). Sur le site contemporain de La Rochette, la présence du poutrage calciné à la base du talus conservé en bordure de promontoire rappelle, en dimensions plus réduites, les techniques de construction du Camp de Péran. La fusion des plaquettes de schiste y est également l'expression de températures élevées. À Péran, les fouilles ont également mis au jour des structures d'habitat au pied interne du rempart, préservées par l'effondrement de ce dernier (constructions de pierres et de bois, puits, silos). Parmi les éléments de céramiques mis au jour à Péran, la lèvre éversée d'un vase trapu (Nicolardot et Guigon, 1991, fig. 17, $n^{\circ} 5$, p. 142) peut être comparée morphologiquement à un exemplaire de la Rochette (ibid., $\mathrm{n}^{\circ}$ 10). À l'instar du site de La Rochette (voir infra, $3^{\mathrm{e}}$ partie), l'analyse paléoenvironnementale du Camp de Péran révèle un milieu forestier de chênes et hêtres, mais en phase régressive avec la présence de noisetiers, aulnes, bouleaux, saules et genêts.

Le Camp des Salles, sur la pente nord de la Montagne du Prieuré en Locronan (Finistère), est constitué de trois enclos juxtaposés et alignés dont la surface totale atteint 3,5 ha et dont les dimensions diminuent progressivement vers le sommet de la colline. Les enclos sont matérialisés soit par des talus dont l'élévation conservée varie de 0,50 à $3,50 \mathrm{~m}$, soit par un remodelage de la pente naturelle pour l'enclos supérieur. Cette implantation sur la pente, permettant un accès facile à partir du sommet de la colline, exclut une fonction militaire et une telle configuration topographique s'oppose nettement de celle du site de la Rochette. Les fouilles menées de 1986 à 1991 dans l'enclos supérieur ont mis au jour un ensemble de neuf bâtiments à soubassements de pierres. Trois datations par le radiocarbone et la présence de deux monnaies situent l'occupation dans le courant du IX siècle (Guigon, 1997). 
Une datation des $\mathrm{VII}^{\mathrm{e}}$-VIII ${ }^{\mathrm{e}}$ siècles, identique à celles de La Rochette, a été obtenue sur l'enceinte de Botalec en Landevant (Morbihan) : Gif-4080 : $1260 \pm 90$ B.P., soit 690-780 apr. J.-C. en âge calibré (Giot, 1981; Guigon, 1997). Cet ouvrage circulaire de 120 à $150 \mathrm{~m}$ de diamètre est bordé d'un talus bas et large au nord et à l'est et d'un muret en pierres sèches au sud. Ce dernier contient des blocs vitrifiés révélant un incendie violent à l'image des sites de Péran et de La Rochette.

L'habitat du haut Moyen Âge de Creac'h-Gwen en Quimper (Finistère), étudié en fouille de sauvetage dans des conditions difficiles en 1986 (Menez et Batt, 1988), est plus récent et daté de la seconde moitié du $\mathrm{x}^{\mathrm{e}}$ siècle. Associé à un réseau dense de fossés et à plusieurs structures de combustion aux fonctions diverses (four à sécher le grain, basfourneau, tranchées-foyers), le plan légèrement trapézoïdal d'un bâtiment sur poteaux $(6,50 \mathrm{~m}$ de large, 7 à $13 \mathrm{~m}$ de long) n'est pas très éloigné de celui de la maison sud de La Rochette et un large foyer est également présent en bordure externe de la paroi orientale.

Le village carolingien de La Cocherais à Tinténiac (Illeet-Vilaine), fouillé en préventif en 1989 (Le Boulanger et Provost, 1992), présente également une structuration de l'espace matérialisée par un réseau de fossés orthonormés dans lequel s'inscrit un ensemble de structures en creux caractéristiques de l'habitat : trous de poteau, bâtiments sur solins et sablières, foyers, silos, fosses détritiques... La présence de petites tranchées limitant un ensemble de trous de poteau (secteur III, ibid. p. 93) rappelle celle marquant la paroi nord du bâtiment central $4 \mathrm{~d}$ de La Rochette. Le mobilier céramique est riche en vases décorés à la molette. L'élément de lèvre oblique éversée de La Rochette (fig. 51, $\mathrm{n}^{\circ}$ 6) s'identifie au vase globulaire $\mathrm{n}^{\circ}$ 6670-6033 de La Cocherais (Boulanger et Provost, 1992, fig. 15) et vient en complément des datations aux VIII ${ }^{\mathrm{e}}$ et $\mathrm{IX}^{\mathrm{e}}$ siècles obtenues par le radiocarbone.

Quelques vestiges d'habitat de la même période ont été mis au jour en 1993 en rive gauche de la Vilaine au lieu-dit Vieuxville-Beaurade au sud-ouest de Rennes (Leroux et al., 1998).

En 1996 et 1997, les fouilles préventives préalables à la réalisation de l'autoroute A 84 entre Rennes et Caen ont permis l'étude, d'une ampleur jusqu'alors inégalée en Bretagne, de quatre habitats carolingiens groupés de la région du Coglais (Catteddu, 2001). Une forte structuration de l'espace sous la forme de parcelles rectangulaires régulières y a été reconnue et la spécialisation de certains secteurs des habitats est attestée par la présence de concentrations de structures excavées (foyers, silos, fours, activité métallurgique...). Le mobilier, essentiellement céramique, est caractéristique de l'époque carolingienne et plusieurs datations par le radiocarbone situent l'occupation de ces habitats entre les $\mathrm{VII}^{\mathrm{e}}$ et $\mathrm{IX}^{\mathrm{e}}$ siècles, période contemporaine de celle de La Rochette. Les études paléoenvironnementales menées autour des sites carolingiens du Coglais révèlent une forte activité agricole mais les charbons de chêne laissent entrevoir la variété des biotopes complémentaires avec, à l'image du site de La Rochette, le maintien de forêts denses au voisinage de milieux plus ouverts.

À La Rochette, l'absence des structures excavées domestiques habituellement mises au jour sur ces sites d'habitat (silos, tranchées-foyers, fours excavés, fosses...) est remarquable, tant dans l'enceinte fortifiée que sur le vaste espace dégagé en partie nord du site. Les seules structures pouvant être associées à la vie quotidienne sont les deux bâtiments à extrémité en abside ( $4 \mathrm{~d}$ et $6 \mathrm{c}$ ), les petits ensembles de trous de poteau isolés au nord-est (greniers? 4e et $4 \mathrm{f}$ ) et quatre structures de combustion à proximité des bâtiments.

La nature du substrat, impropre à une exploitation comme matériau, peut expliquer l'absence de fosses tandis que la destruction violente et massive des structures par incendie peut justifier une durée d'occupation trop brève pour voir se développer les traces d'une vie domestique. Toutefois, la position topographique et l'aspect défensif du site révèlent son statut particulier - résidence aristocratique, site refuge temporaire en cas de conflit, poste de contrôle de la vallée et des voies de passage - qui ainsi se distinguerait de celui des sites à vocation domestique. On notera à ce sujet que le volume des bâtiments de La Rochette - de la maison sud $6 c$ notamment avec une surface au sol dépassant $75 \mathrm{~m}^{2}$ - se démarque de ceux d'habitats contemporains, les surfaces variant de 20 à $50 \mathrm{~m}^{2}$ au maximum sur les sites de Montours ou La Chapelle-Saint-Aubert par exemple. La présence d'une probable galerie en façade du bâtiment de La Chapelle-Saint-Aubert rappelle les trois poteaux alignés en façade du bâtiment sud 6c.

Si le plan de la maison 6c de La Rochette apparaît original par rapport aux maisons habituellement connues au haut Moyen Âge (Chapelot et Fossier, 1980), une certaine similitude avec l'habitat carolingien des Sureaux à La GrandeParoisse (Seine-et-Marne) (Petit, 2009) peut être évoqué. Sur ce site de la fin du IX ${ }^{\mathrm{e}}$-début du $\mathrm{X}^{\mathrm{e}}$ siècle, le plan et les dimensions de plusieurs bâtiments sur poteaux, rectangulaires et à trois ou quatre nefs suivant les cas, sont comparables à ceux du bâtiment $6 c$. La proposition de restitution (Petit, 2009, p. 35) d'un "bâtiment à quatre nefs, toiture à pans coupés et pignons apparents en partie haute " pourrait s'appliquer à la maison de La Rochette en raison de sa structure centrale carrée aux fondations plus puissantes (voir supra, $\mathbb{S}$ IX D). On notera également la présence aux Sureaux de greniers à extrémité en $\mathrm{V}$ et d'une tour-porche en entrée de l'enceinte fossoyée, étayant l'hypothèse de struc- 
tures d'accès et de surveillance comparables dans l'enceinte en bois de La Rochette. Sur le plan paléoenvironnemental en revanche, les analyses anthracologiques diffèrent sensiblement : aux Sureaux, le hêtre est présent dès l'occupation du Bronze final et a tendance à être supplanté par le chêne, contrairement à son développement plus tardif en Bretagne (voir infra, $3^{\mathrm{e}}$ partie). Cependant, le phénomène général d'ouverture du milieu, notamment aux périodes historiques, est perceptible sur les deux sites.

La destruction systématique et brutale de l'enceinte et du bâtiment de cette phase 6 de La Rochette est peut-être liée au contexte historique de la transition entre les époques mérovingienne et carolingienne qui se caractérise par d'importants changements politiques, comme la mise en place par les Carolingiens de la Marche de Bretagne pour contenir les Bretons. De nombreuses expéditions militaires contre les Bretons sont menées au cours des viII et $\mathrm{IX}^{\mathrm{e}}$ siècles. Ainsi, sur la commune voisine de Gaël, le monastère fondé par saint Méen, dont la localisation reste indéterminée, fut incendié à la fin du viII siècle ou au début du suivant, puis restauré sur ordre de Louis le Pieux (Guigon, 1993). Dans un tel contexte, la position du promontoire de La Rochette, légèrement en retrait d'un carrefour de voies antiques et à quelques centaines de mètres en aval d'un lieu de franchissement de l'Yvel, a eu certainement une influence déterminante sur le choix du site.

\section{Troisième partie \\ ÉTUDES ANTHRACOLOGIQUES SUR LES STRUC- tures, du NéOlithiQue aU haut Moyen ÂGe (N. M.)}

\section{Introduction}

Bon nombre de charbons de bois ont été mis au jour lors de la fouille du site de La Rochette. Ces prélèvements apparaissent particulièrement intéressants en raison de la succession de quatre occupations, du Néolithique ancien au haut Moyen Âge. Les résultats de l'analyse anthracologique ont ainsi pu être comparés de façon diachronique, donnant à la fois des indications sur les ligneux présents dans l'environnement du site et sur les choix des bois utilisés. La précision de la chronologie des lots apporte une plus-value aux données anthracologiques, qui pourront servir de références pour les études ultérieures.

Deux objectifs ont été définis pour cette étude. Le premier, d'ordre technique et culturel, visait la détermination des essences utilisées pour les différents usages au fil du temps, ainsi que leurs caractéristiques. Pour ce volet, un effort particulier a porté sur la mesure des cernes annuels de croissance du chêne dont la largeur est étroitement liée au contexte environnemental au sein duquel l'arbre a crû. Le second objectif visait à reconstituer l'environnement paysager autour du site au moment des différentes occupations. Les données anthracologiques provenant pour la plupart de charbons de bois issus de constructions (bâtiments, palissade), elles ne permettent pas à elles seules ces reconstitutions. Ainsi, des données polliniques de sites proches ont été mises à contribution pour ce deuxième objectif.

\section{Matériel et MÉTHOdeS}

Les prélèvements ont été effectués soit directement au moment de la fouille, soit au tamisage de sédiments extraits en masse. Au laboratoire, un inventaire de ces prélèvements a été dressé. La stratégie d'analyse a été définie en fonction des objectifs de l'étude.

Tous les lots étudiés sont des sous-échantillonnages des prélèvements initiaux. Comme le chêne est le taxon largement dominant et que les structures échantillonnées sont des constructions, le sous-échantillonnage aléatoire en vue d'une reconstitution environnementale selon la méthode habituelle n'a pas semblé adapté. En effet, comme cette méthode implique l'analyse de charbons de toutes tailles, bon nombre de ceux-ci auraient été du chêne et les plus petits charbons n'auraient pas permis de faire les observations macroscopiques et dendrométriques, riches d'informations. Le sous-échantillonnage a donc porté sur les gros et moyens fragments, avec une recherche visuelle à la loupe binoculaire des charbons d'aspects différents pour identifier le plus possible de taxons. Les charbons qui restent sont conservés au laboratoire d'anthropologie et archéométrie de l'UMR 6566 et pourront faire l'objet d'analyses complémentaires.

L'observation des charbons de bois s'effectue à la loupe binoculaire (grossissement $7 \mathrm{X}$ à $90 \mathrm{X}$ ) et au microscope (X100, X200 et X500). L'identification se base sur l'observation des trois coupes anatomiques du bois, transversale, tangentielle et radiale. Les caractéristiques anatomiques de l'échantillon sont relevées. Une clé de détermination (Schweingruber, 1982, 1990), et si nécessaire l'observation d'échantillons actuels, permet la détermination du taxon.

La détermination se fait la plupart du temps au niveau du genre. Par exemple, le chêne rouvre ne peut être différencié du chêne pédonculé; ces deux espèces sont comprises dans le taxon Chêne (Quercus). Certains genres sont difficilement différenciés; ainsi, les sorbiers (Sorbus), poiriers et 
pommier (Pyrus et Malus), les aubépines (Crataegus) et le néflier (Mespilus germanica) sont regroupés dans le taxon Pomoïdées. De même, le taxon Fabacées regroupe les genêts (Cytisus scoparius, Genista sp.) et les ajoncs (Ulex sp.). Enfin, le chêne et le châtaignier se différencient sur la base d'un seul critère, la présence de rayons ligneux multisériés chez le premier; si ce critère ne peut être observé correctement, en raison souvent de la taille réduite du charbon, l'échantillon est classé dans le taxon Chêne/Châtaignier.

Outre la détermination du taxon, l'analyse anthracologique comporte d'autres observations (Marguerie et Hunot, 2007). La courbure des cernes de croissance est qualifiée : une faible courbure correspond à un bois de gros calibre (diamètre approximatif $>20 \mathrm{~cm}$ ); une forte courbure indique la proximité de la moelle donc un bois de petit calibre (diamètre approximatif $<8 \mathrm{~cm}$ ) ou le cœur d'un bois de calibre intermédiaire ou gros et une courbure intermédiaire indique un calibre moyen (diamètre approximatif $8-20 \mathrm{~cm}$ ) ou une partie interne d'un bois de gros calibre. Dans le cas du chêne, la présence de thylles (excroissances cellulaires bouchant les vaisseaux dans le bois de cœur ou duramen) permet de déterminer s'il s'agit de calibres petits et intermédiaires provenant d'une grosse pièce de bois.

Au cours de l'analyse anthracologique, la largeur des cernes de croissance annuelle est mesurée; il s'agit de la largeur totale divisée par le nombre de cernes observés. Ces données permettent d'apprécier les conditions de croissance des ligneux. Des cernes larges indiquent une croissance peu contrainte voire libre, tandis que des cernes étroits sont le reflet d'une croissance contrainte, par exemple d'arbres poussant en forêt dense ou sénescents. Deux charbons de chêne comportant plus de trente cernes successifs ont été réservés pour une étude dendrochronologique.

Enfin, les aspects particuliers des charbons sont notés : fendu (présence de fentes de retrait) et/ou dur/luisant (éléments anatomiques partiellement fondus). Ces observations renseignent sur les conditions de combustion des bois, entre autres la vitesse de montée en chaleur (Théry-Parisot, 2001; Marguerie et Hunot, 2007). La présence de galeries d'insectes xylophages indique qu'il s'agit de bois sénescents ou morts.

\section{RÉSULTATS ET INTERPRÉTATION}

Les résultats ont été compilés en dénombrements uniquement : pour ce type d'étude à très forte prédominance du chêne et à partir de structures de constructions, les masses n'apportent aucune information supplémentaire. Un diagramme anthracologique de fréquences de dénombrement (fig. 52) est proposé pour offrir une vue synthétique des résultats par période. Compte-tenu de la stratégie adoptée, l'analyse est basée sur la présence-absence des taxons et non leur fréquence relative. C'est la raison pour laquelle les résultats par période sont présentés sous forme de tableaux de présence (fig. 53 et infra, fig. 56 et 59).

Les largeurs de cernes sont présentés sous forme d'histogrammes, les classes de largeurs étant par tranches de

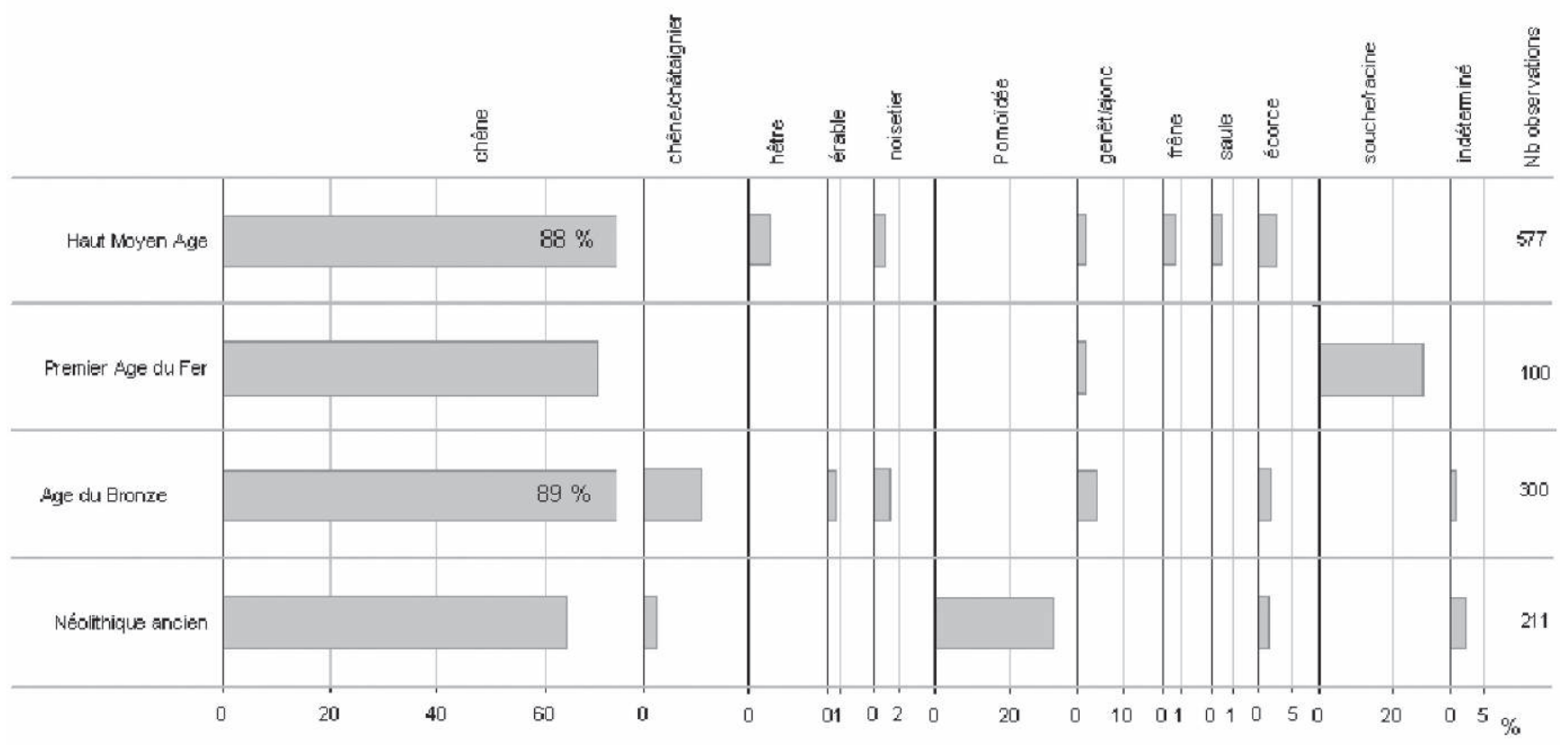

Figure 52 : Pourcentages de dénombrement des charbons identifiés par ensemble archéologique.

Figure 52: Frequency of charcoal number in each archaeological whole. 


\begin{tabular}{|c|c|c|c|c|}
\hline & & & masse totale prélevée $(\mathrm{g})$ & nombre charbons analysés \\
\hline \multirow{13}{*}{ haut Moyen Âge } & \multirow{2}{*}{ talus } & TLU 3 & & 18 \\
\hline & & TLU 4 & & 151 \\
\hline & \multirow{9}{*}{ double palissade sud } & ТP 477 & 50 & 40 \\
\hline & & TP 478 & 40 & 30 \\
\hline & & ТР 479 & 150 & 38 \\
\hline & & ТР 481 & 150 & 60 \\
\hline & & ТР 482 & 65 & 25 \\
\hline & & ТР 483 & 40 & 25 \\
\hline & & ТP 485 & 135 & 30 \\
\hline & & TP 544 & 80 & 30 \\
\hline & & TP 549 & 40 & 30 \\
\hline & \multirow{2}{*}{ bâtiment sud } & ТР 620 & 6 & 30 \\
\hline & & ТР 621 & 15 & 70 \\
\hline premier âge du Fer & foyer & fo. 09 & 75 & 100 \\
\hline \multirow{9}{*}{ âge du Bronze } & \multirow{3}{*}{$\begin{array}{l}\text { habitat } \\
1^{\text {re }} \text { entrée ouest }\end{array}$} & ТP 112 & 5 & 24 \\
\hline & & TP 144 & 8 & 56 \\
\hline & & ТР 146 & 4 & 20 \\
\hline & \multirow{6}{*}{$\begin{array}{c}\text { habitat } \\
2^{\mathrm{e}} \text { entrée ouest }\end{array}$} & ТР 154 & 25 & 60 \\
\hline & & ТP 155 & 40 & 30 \\
\hline & & TP 157 & 50 & 40 \\
\hline & & ТР 159 & 7 & 20 \\
\hline & & TP 166 & 8 & 20 \\
\hline & & ТР 167 & 7 & 30 \\
\hline Néolithique ancien & foyer & fo. 06 & 50 & 211 \\
\hline
\end{tabular}

Tableau 4 : Masse totale des charbons prélevés et nombre de charbons analysés par structure.

Table 4: Total sampled mass of charcoal and number of charcoal fragments analysed in each structure.

0,25 mm. Moyennes et écarts-types ont été calculés par lot et par période. Notre stratégie de sous-échantillonnage ayant privilégié l'observation des charbons de moyenne et grande taille, les mesures de largeurs de cerne sur le chêne sont très nombreuses et permettent une analyse détaillée par structure.

\section{A. Le site dans sa globalité}

Sur l'ensemble du site, vingt-quatre structures ont fait l'objet d'analyses (tabl. 4) : vingt trous de poteau, deux prélèvements en talus et deux foyers. Le Néolithique ancien est représenté par un foyer (fo.06), l'âge du Bronze par neuf trous de poteau des première et deuxième entrées ouest, le premier âge du Fer par un foyer (fo.09), et le haut Moyen Âge par onze trous de poteau appartenant au bâtiment sud et à la palissade sud, plus deux prélèvements en talus. Au total, 1188 charbons de bois ont été observés, avec un minimum de vingt charbons par structure. L'objectif de cent charbons minimum par unité de construction a été atteint.
Le cortège des ligneux est peu diversifié sur le site. Neuf taxons différents ont été identifiés : le chêne, le chêne/châtaignier, le hêtre, l'érable, le noisetier, des Pomoïdées, des Fabacées, le frêne et le saule. Le chêne, le chêne/châtaignier et le hêtre sont les grands arbres des forêts du Massif armoricain, chênaies ou chênaies-hêtraies. L'érable, le noisetier et les Pomoïdées peuvent être des composantes du sous-étage forestier; les deux premières espèces et certaines Pomoïdées sont, pour le Massif armoricain, le reflet de conditions stationnelles riches difficilement envisageables sur l'éperon même, composé de schiste briovérien particulièrement dur et acide. Ces trois taxons, tout comme les Fabacées, sont héliophiles et peuvent aussi occuper les lisières forestières et les milieux ouverts. Le frêne et le saule occupent les stations alluviales en bordure de cours d'eau, le premier étant particulièrement exigeant quant à la richesse minérale du sol.

Outre ces ligneux bien définis, certains lots comportent des écorces dont on ne peut déterminer l'espèce de provenance. Et plusieurs charbons du foyer du premier âge du Fer (fo.09), aux fibres torses, aux zones poreuses mal définies, 


\begin{tabular}{|c|c|c|c|c|}
\hline & Néolithique ancien & Âge du Bronze & Premier Âge du Fer & Haut Moyen Âge \\
\hline & f 06 & & f 09 & \\
\hline \multicolumn{5}{|l|}{ chêne } \\
\hline \multicolumn{5}{|c|}{ chêne/châtaignier } \\
\hline \multicolumn{5}{|l|}{ hêtre } \\
\hline \multicolumn{5}{|l|}{ érable } \\
\hline \multicolumn{5}{|l|}{ noisetier } \\
\hline \multicolumn{5}{|l|}{ Pomoïdée } \\
\hline \multicolumn{5}{|l|}{ Fabacées } \\
\hline \multicolumn{5}{|l|}{ frêne } \\
\hline \multicolumn{5}{|l|}{ saule } \\
\hline \multicolumn{5}{|l|}{ écorce } \\
\hline \multicolumn{5}{|l|}{ souche/ racine } \\
\hline indéterminé & & & & \\
\hline
\end{tabular}

$1=\square, 2=\square, 3=$

Figure 53 : Présence des taxons par période (1, majoritaire; 2 , abondant; 3 , présence notable 4, faible présence 5 , rare). Figure 53: Presence of taxa by periods (1, major; 2, abundant; 3, notable; 4,faint; 5, rare).

aux aspects de loupes (fibres noueuses, enchevêtrées) ont été regroupés sous l'appellation souche/racine, faute de référentiel pour ce type de bois.

\section{B. Bilan par phases d'occupation}

\section{B 1. Le Néolithique ancien}

La structure analysée est un foyer (fo.06); 211 charbons y ont été observés. Le chêne est le taxon dominant, accompagné de Pomoïdées et de quelques fragments d'écorce (fig. 52, 53). Les charbons de chêne et de Pomoïdées proviennent pour une part de bois de gros calibre mais ce sont surtout les calibres intermédiaire et petit qui sont représentés (fig. 54). Lobservation fréquente de thylles sur les charbons de chêne indique que ce lot se compose d'une bonne proportion de bois de coeur. Quelques galeries d'insectes ont été observées sur les chênes (17 cas sur 135 fragments), tandis qu'elles sont fréquentes chez les Pomoïdées (37 sur 67). Quelques chênes ont un aspect fendu. Les cernes de croissance du chêne sont étroits, avec une moyenne de 0,94 $\pm 0,56 \mathrm{~mm}$ pour la période (fig. 55 en bas). Les largeurs sont bien classées autour de la moyenne, indiquant une même contrainte de croissance pour l'ensemble du lot.

Ces résultats indiquent dans l'environnement proche une chênaie à forte contrainte de croissance, en lien probablement avec la densité du peuplement forestier et possiblement accentuée par la pauvreté minérale du sol. Le sous-étage forestier se compose de petits fruitiers et, dans l'hypothèse d'un prélèvement au sein d'une même forêt, le poirier à feuilles en cœur est le plus probable en raison de sa frugalité. La présence de ce(s) fruitier(s) marque une certaine sécheresse du lieu de prélèvement. Ces arbres et arbustes ont pu être prélevés sur l'éperon même.

\section{B 2. L'âge du Bronze}

Les échantillons de cette période proviennent de trous de poteau composant les première et deuxième entrées ouest de la structure 2 d. 100 charbons ont été observés pour la première entrée et 200 pour la seconde. Le taxon dominant est le chêne (fig. 52, 53, 56), accompagné du genêt/ajonc, du noisetier, et de l'érable (dans le TP.166 uniquement). Des écorces ont été observées dans le TP.154.

Les charbons de chêne proviennent de quelques pièces de gros calibre mais surtout de pièces de calibre intermédiaire (fig. 54). Peu de thylles ont été observés (15 cas sur 268), traduisant la rareté du bois de cœur dans le prélèvement. La moyenne des largeurs de cerne du chêne est plus du double de celle mesurée au Néolithique ancien, soit 2,14 \pm $0,82 \mathrm{~mm}$. L'histogramme des largeurs de cernes est aussi beaucoup plus étalé, avec des valeurs allant jusqu'à $4 \mathrm{~mm}$ (fig. 55). Deux groupes de valeurs semblent se dessiner : un premier centré sur 1,5-2 mm et un deuxième, partiellement superposé et centré sur 2,5-3 mm. Les chênes ayant servi à la construction de ces deux entrées ont vraisemblablement été soumis à deux niveaux de contrainte : pour certains, une croissance contrainte, bien qu'à un degré moindre qu'au Néolithique ancien, et une croissance plus libre pour les autres, conséquence possible d'une ouverture du milieu.

La présence du genêt/ajonc à cette période corrobore cette interprétation. Certains charbons de chêne comportent des 


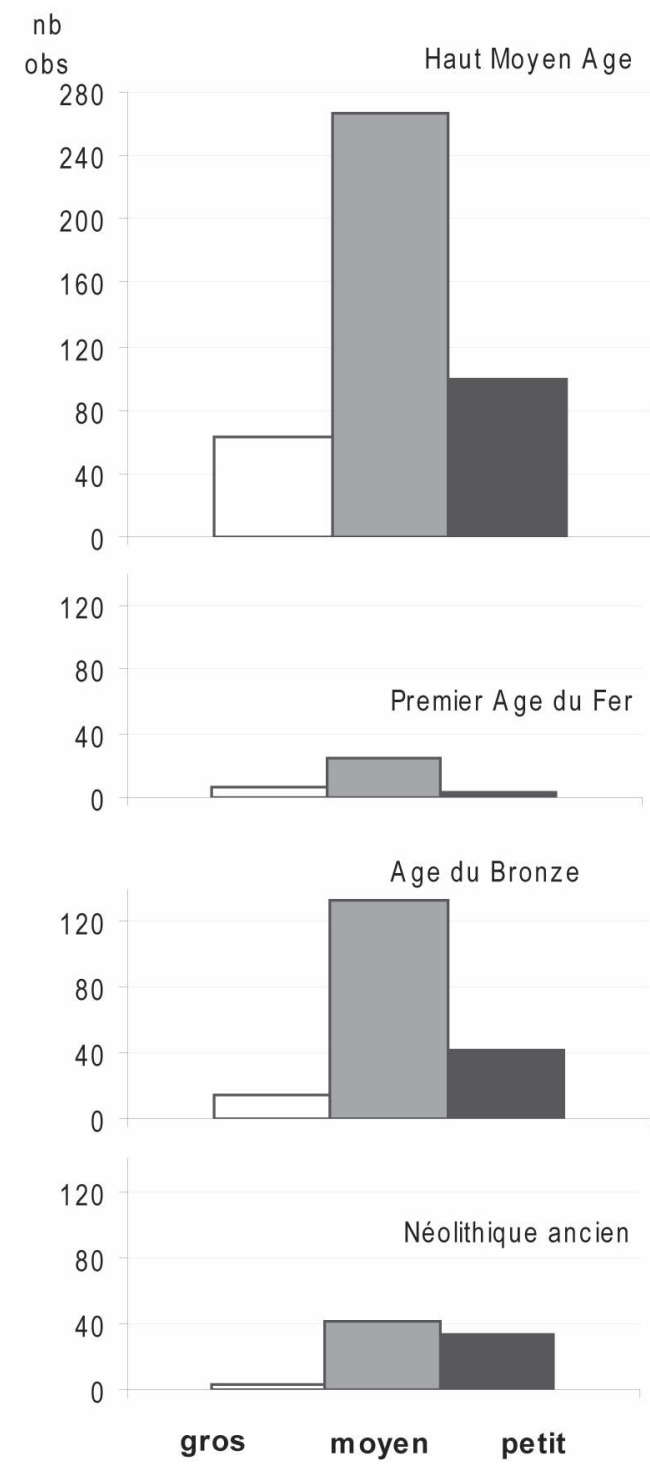

CALIBRES

\begin{tabular}{|l|c|c|}
\hline & $\begin{array}{c}\text { nb obs. } \\
\text { thylles }\end{array}$ & $\begin{array}{c}\text { nb total } \\
\text { chêne }\end{array}$ \\
\hline Haut Moyen Age & 136 & 506 \\
\hline Premier Age du Fer & 8 & 70 \\
\hline Age du Bronze & 15 & 268 \\
\hline Néolithique ancien & 69 & 135 \\
\hline
\end{tabular}

Figure 54 : Fréquence des différents calibres (gros, intermédiaire, petit) et des thylles sur les charbons de chêne.

Figure 54: Frequency of the different sizes (large, middle, small) and thylles presence on oak charcoals. galeries d'insectes (39/268) ou ont un aspect particulier : fendu, luisant, mais surtout fendu/luisant (fig. 57). Les charbons d'érable proviennent de bois de calibre intermédiaire, ceux de noisetier de calibres intermédiaire et petit, et tous les charbons de genêt/ajonc sont de petit calibre.

Du point de vue technologique, le chêne devait être la meilleure essence disponible autour du site pour la réalisation de poteaux; des bois de calibre intermédiaire ont vraisemblablement été préférés. Le chêne est un arbre de haut fût. Son bois est d'excellente qualité et très durable, en particulier le bois de cœur. Il est utilisé couramment comme bois d'œuvre pour les charpentes, les constructions diverses et en menuiserie. Les bois de petit calibre de noisetier et de genêt/ajonc sont souples et ont pu être utilisés dans les constructions pour la fabrication de clayonnages.

Des différences de détail peuvent être relevées entre les deux entrées. La première entrée ouest n'a livré que deux taxons, le chêne et le genêt/ajonc, tandis que la seconde comporte aussi du noisetier et de l'érable (fig. 56). Les charbons de la première entrée sont fendus et fendus/luisants, ils sont luisants et surtout fendus/luisants dans la deuxième. Trois trous de poteau contiennent des charbons provenant de pièces de chêne de gros calibre : les TP.144, 154 et 157 . Les six autres ne comportent que des chênes de calibres intermédiaire et petit. Les histogrammes de classes de largeur de cernes par trou de poteau montrent que les lots de charbons se situent dans l'un ou l'autre des deux groupes de niveaux de contrainte de croissance, à l'exception de ceux des TP. 144, 154 et 167, qui couvrent toute l'étendue de la variation (fig. 58). Les histogrammes de l'une et l'autre entrée présentent le même étalement, reflétant une homogénéité dans la configuration du ou des peuplements forestiers d'origine.

A l'âge du Bronze, les chênes ont une contrainte de croissance qui s'est allégée comparativement au Néolithique ancien. La chênaie est toujours présente comme le montre le premier groupe de largeur de cernes centré sur 1,5-2 mm, mais sa configuration a changé. Soit elle est moins dense, avec des zones de croissance quasi libre, soit les bois ont été recueillis dans un endroit différent de ceux du Néolithique ancien. Ces deux états sont probablement présents dans l'environnement du site, mais la deuxième hypothèse paraît la plus plausible ici. En effet, les bois du Néolithique ancien pouvaient tout à fait se trouver sur l'éperon rocheux (station pauvre et sèche). Par contre, les essences accompagnant le chêne à l'âge du Bronze, à savoir le noisetier et l'érable, ont des exigences plus grandes en matière de richesse minérale $\mathrm{du}$ sol et leur présence sur l'éperon semble peu plausible. L'éperon était d'ailleurs vraisemblablement défriché à cette période, comme le laissent penser les vestiges archéologiques. L'approvisionnement en bois pour la construction 
Figure 55: Largeurs de cernes mesurées sur les charbons de chêne : histogrammes par période d'occupation avec moyennes et écarts-types (classes en $\mathrm{mm}$ ). Figure 55 : Rings width measured on oak charcoals by periods of occupation, with mean and standard deviation (histogram classes in $\mathrm{mm}$ ).

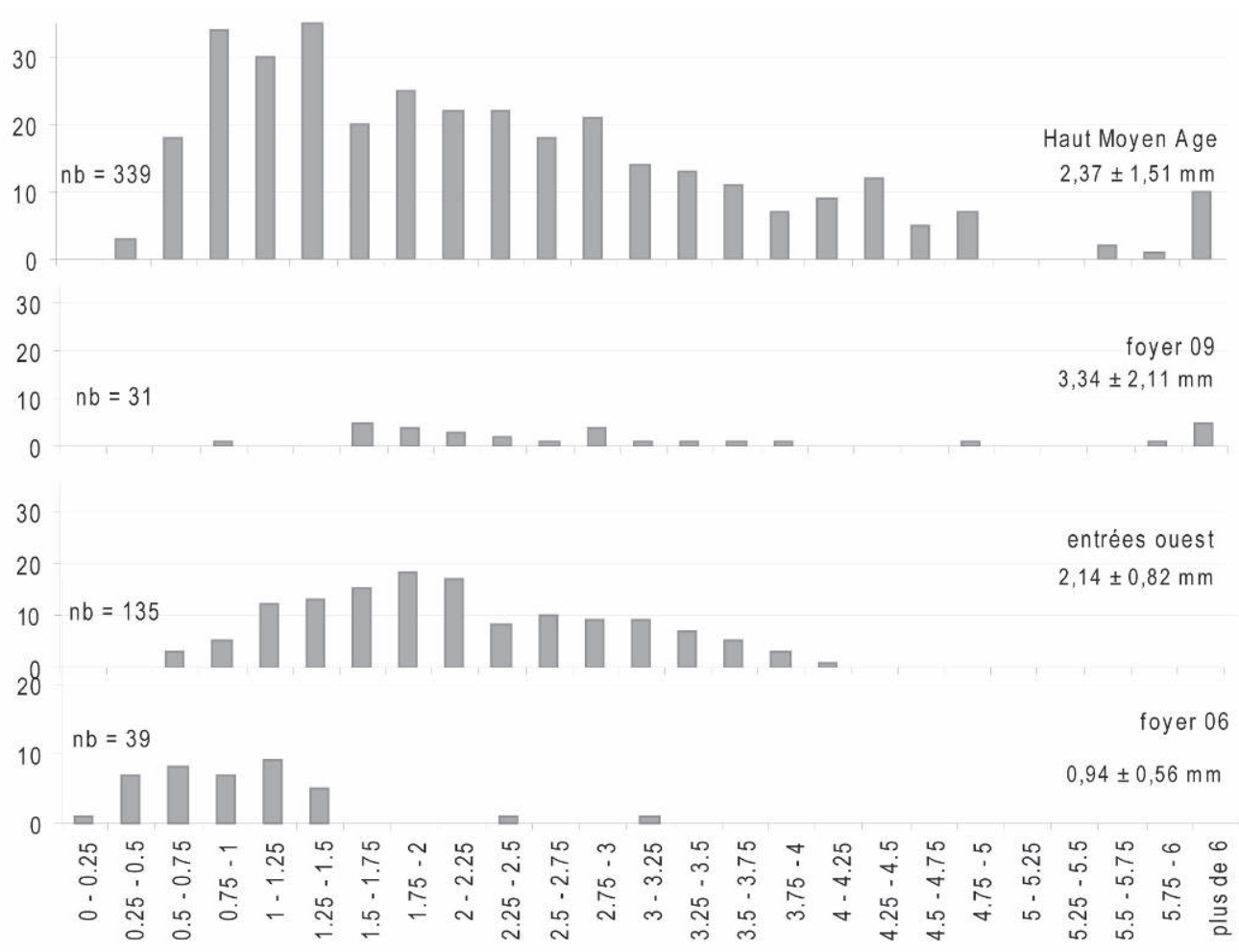

\begin{tabular}{|c|c|c|c|c|c|c|c|c|c|}
\hline & \multicolumn{3}{|c|}{$1^{\text {re }}$ entrée ouest } & \multicolumn{6}{|c|}{$2^{\mathrm{e}}$ entrée ouest } \\
\hline & TP 112 & TP 144 & TP 146 & ТР 154 & TP 155 & ТР 157 & ТР 159 & ТР 166 & TP 167 \\
\hline \multicolumn{10}{|l|}{ chêne } \\
\hline \multicolumn{10}{|c|}{ chêne/châtaignier } \\
\hline \multicolumn{10}{|l|}{ hêtre } \\
\hline \multicolumn{10}{|l|}{ érable } \\
\hline \multicolumn{10}{|l|}{ noisetier } \\
\hline \multicolumn{10}{|l|}{ Pomoïdée } \\
\hline \multicolumn{10}{|l|}{ Fabacées } \\
\hline \multicolumn{10}{|l|}{ frêne } \\
\hline \multicolumn{10}{|l|}{ saule } \\
\hline \multicolumn{10}{|l|}{ écorce } \\
\hline \multicolumn{10}{|l|}{ souche/ racine } \\
\hline indéterminé & & & & & & & & & \\
\hline
\end{tabular}

$$
1=\square, 2=\square, 3=\square, 4=\square, 5=
$$

Figure 56 : Âge du Bronze : présence des taxons par structure (1, majoritaire; 2, abondant; 3, présence notable; 4, faible présence; 5 , rare).

Figure 56: Bronze Age: taxa presence by structure (1, major; 2, abundant; 3, notable; 4,faint; 5, rare).

des deux entrées ouest s'est, dans ce contexte, effectué en périphérie du site, dans des zones plus riches de bas de pente par exemple.

\section{B 3. Le premier âge du Fer}

Les charbons de bois du premier âge du Fer proviennent d'un seul foyer (fo.9). Compte-tenu de l'homogénéité du lot, un sous-échantillonnage de cent charbons s'est avéré 


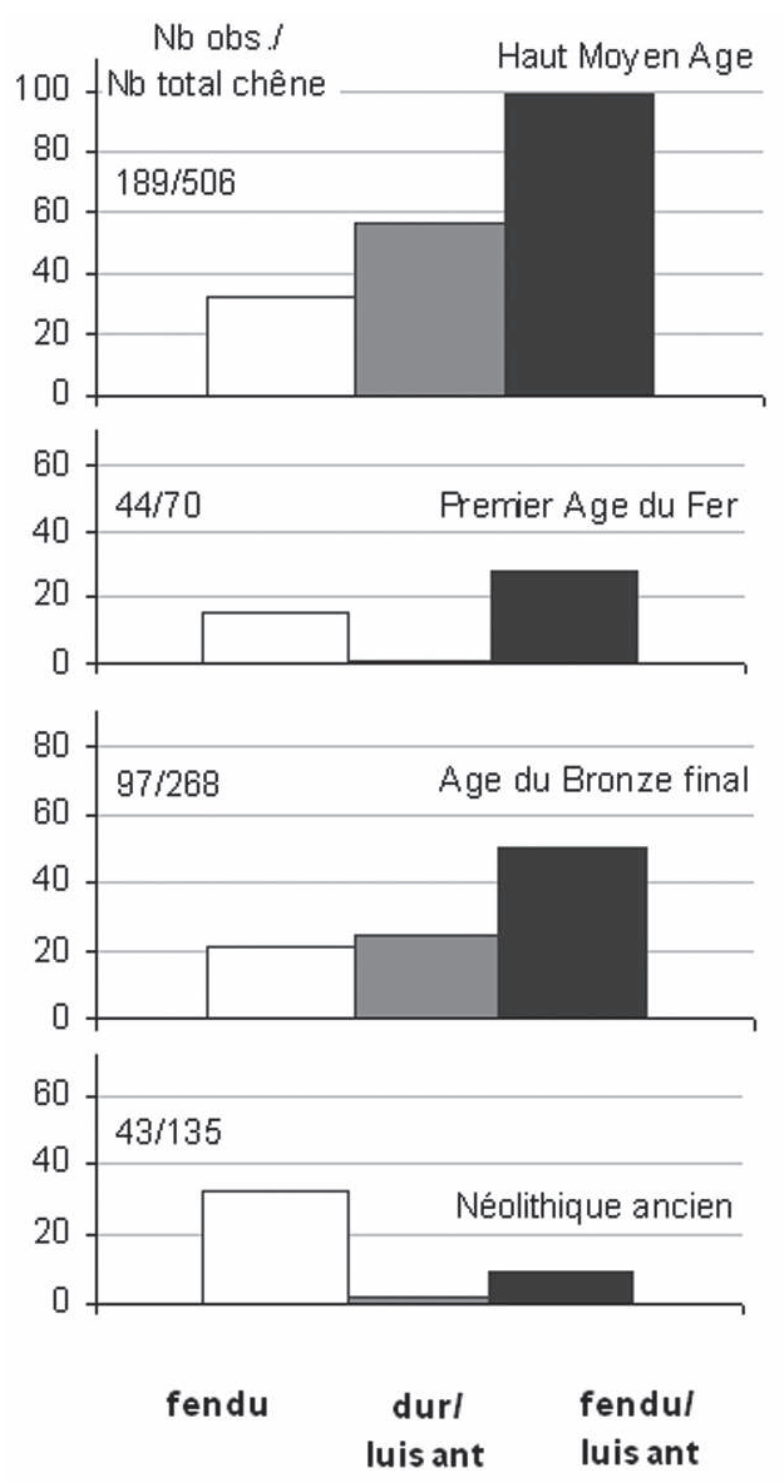

Figure 57 : Nombre d'observation des aspects particuliers (fendu, dur/luisant, fendu/luisant) sur les charbons de chêne (en cartouche, nombre d'observations / nombre total de charbons de chêne).

Figure 57: Observed number of particular aspects on oak charcoal (splitted, hard/glossy, splitted/glossy).

représentatif. Le chêne est le taxon dominant (fig. 52, 53), accompagné de Fabacées (genêt/ajonc). La caractéristique particulière de ce lot est la présence notable de bois aux fibres torses, aux zones poreuses mal définies, aux aspects de loupes (fibres noueuses, enchevêtrées). Il s'agit probablement de chêne mais, faute de référentiel, ces charbons ont été regroupés sous l'appellation souche/racine.

Pour le chêne, de gros calibres sont présents ainsi que des calibres intermédiaires (fig. 54). Peu de petits calibres ont été observés. Ces bois comportent rarement des thylles; le

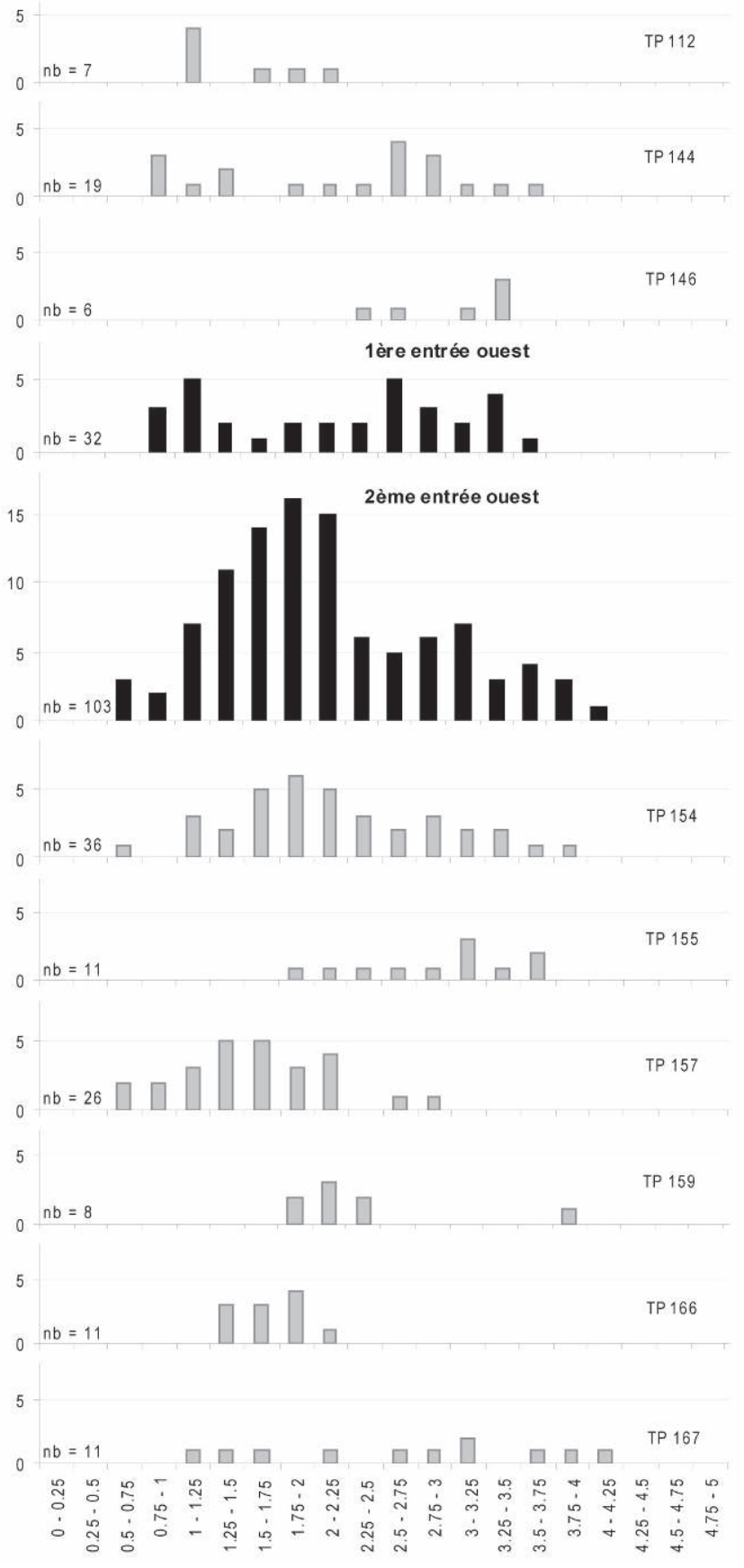

Figure 58 : Âge du Bronze : histogrammes des largeurs de cernes mesurées sur les charbons de chêne par structure.

Figure 58: Bronze Age: Rings width of oak charcoals, by structure.

lot est donc composé de peu de duramen. Les galeries d'insectes sont rares. Une bonne partie des charbons de chêne a un aspect fendu (fig. 57), plusieurs sont fendus et luisants, en particulier ceux du lot souche/racine. La haute fréquence de fentes radiales est étroitement liée dans ce cas à la densité élevée des rayons ligneux qui sont larges. Les cernes de croissance annuelle sont très larges, avec une moyenne de 
3,34 mm, et les mesures très étalées (fig. 55), avec un écarttype de $2,11 \mathrm{~mm}$.

Ce lot est celui qui livre le moins d'informations sur l'environnement du site. Ce foyer a pu être alimenté par un seul et même arbre, un chêne qui aurait poussé par exemple en croissance libre sur l'éperon et qui aurait été victime d'un chablis, la totalité de l'arbre étant ainsi accessible : tronc, branches, racines. Les Fabacées, genêt ou ajonc, devaient être largement présents sur le site que l'on sait déjà défriché au Bronze final, d'autant plus s'il a été abandonné entre les deux occupations mises en évidence, laissant libre cours à l'implantation de ces ligneux héliophiles.

\section{B 4. Le haut Moyen Âge}

Les charbons de bois datant du haut Moyen Âge ont trois origines : le bâtiment sud (deux trous de poteau, 100 charbons analysés), la double palissade sud (neuf trous de poteau, 308 charbons analysés) et le talus sud (deux ensembles prélevés, 169 charbons analysés). Au total, 577 charbons ont été observés pour cette période.

Six ligneux différents ont été identifiés dans ces structures (fig. 52, 53). Le chêne est le taxon dominant, accompagné du hêtre, du noisetier, de Fabacées (genêt/ajonc), du frêne, du saule. Des écorces ont été observées dans sept des treize structures. La richesse taxonomique par structure est faible (fig. 59), ce qui est habituel pour des cortèges attribués à des constructions; sept des treize structures sont monospécifiques (chêne), quatre comportent deux taxons et deux montrent trois taxons. La double palissade sud est l'ensemble qui présente la plus grande richesse taxonomique (chêne, hêtre, noisetier, frêne et saule). Le cortège du bâtiment sud se compose du chêne, de hêtre, et du genêt/ajonc; les charbons du talus sont tous du chêne.

Le chêne et le hêtre sont des arbres de haut fût ayant une bonne résistance mécanique mais, à la différence du chêne, le hêtre a une mauvaise durabilité extérieure. Sur le site de La Rochette, c'est la structure TP.621 du bâtiment sud qui comporte le plus grand nombre de charbons de hêtre. Ce ne sont pas des pièces de gros calibre qui ont été utilisés mais plutôt des calibres intermédiaires ( 7 des 32 observations, largeur moyenne des cernes $2,46 \mathrm{~mm}$ ) et petits ( $22 \mathrm{des}$ 32 observations), ces derniers peut-être pour la fabrication de chevilles.

Le frêne observé dans la structure TP.549 est de gros calibre; le bois de frêne a une bonne résistance mécanique, notamment en flexion. Le noisetier, le saule et le genêt/ajonc sont aussi des bois souples; ils ont pu être utilisés dans la fabrication de clayonnages.

Par rapport au cortège de l'âge du Bronze, trois taxons sont nouveaux dans cet ensemble du haut Moyen Âge : le hêtre, le frêne et le saule. Le hêtre est récent dans le paysage; en effet, dans sa migration postglaciaire depuis l'est, il est arrivé tardivement dans le Massif armoricain, au cours de l'âge du Bronze. Il a probablement été freiné par les conditions climatiques qui ne lui seyaient guère et par la farouche compétition avec d'autres éléments forestiers plus expansifs, tels le tilleul, le charme et l'érable. Le diagramme pollinique de la forêt de Paimpont montre une courbe continue du

\begin{tabular}{|c|c|c|c|c|c|c|c|c|c|c|c|c|c|}
\hline & bât. sud & \multicolumn{7}{c|}{ double palissade sud } & \multicolumn{2}{c|}{ talus } \\
\hline & 620 & 621 & 477 & 478 & 479 & 481 & 482 & 483 & 485 & 544 & 549 & TLU3 & TLU4 \\
\hline chêne & & & & & & & & & & & & \\
\hline chêne/châtai. & & & & & & & & & & & & & \\
\hline hêtre & & & & & & & & & & & & & \\
\hline érable & & & & & & & & & & & & & \\
\hline noisetier & & & & & & & & & & & & & \\
\hline Pomoïdée & & & & & & & & & & & & & \\
\hline Fabacées & & & & & & & & & & & & & \\
\hline frêne & & & & & & & & & & & & & \\
\hline saule & & & & & & & & & & & & & \\
\hline écorce & & & & & & & & & & & & & \\
\hline souche/ racine & & & & & & & & & & & & & \\
\hline indéterminé & & & & & & & & & & & & & \\
\hline
\end{tabular}

$$
1=\varpi, 2= \pm, 3= \pm, 4=\varpi, 5=
$$

Figure 59 : Haut Moyen Âge : présence des taxons par structure (1, majoritaire; 2, abondant; 3, présence notable; 4, faible présence; 5 , rare).

Figure 59: Early Mediaeval: taxa presence by structure (1, major; 2, abundant; 3, notable; 4,faint; 5, rare). 
hêtre à partir de 725 av. J.-C. environ; ce ne serait qu'à partir de cette date que le hêtre serait réellement entré régionalement en compétition avec le chêne pour composer les formations forestières actuellement typiques du Massif armoricain, les chênaies-hêtraies. Compte-tenu de la date de l'occupation du site de La Rochette au haut Moyen Âge, le hêtre semble avoir été exploité dès son implantation massive dans la région. Le frêne est, quant à lui, très exigeant en ce qui concerne la richesse minérale de sa station et il est probable qu'il ait profité de l'enrichissement des sols en lien avec les activités agropastorales. Constituant un bon fourrage pour l'alimentation des animaux, il a aussi pu être favorisé par l'intervention de l'homme. Quelles qu'en soient la ou les raisons, les données paléobotaniques enregistrent régionalement une diffusion de cet arbre à la fin du Néolithique.

Pour le haut Moyen Âge, toutes structures confondues, le chêne comporte un certain nombre de pièces de gros calibres (fig. 54). Toutefois, la quantité importante des calibres intermédiaires observés laisse à penser qu'il ne s'agit pas uniquement de la partie intérieure des gros bois mais que des pièces de moyen diamètre ont aussi été utilisées. Ce calibre intermédiaire apparaît particulièrement notable au sein du talus (fig. 60, en bas), tandis que la double palissade comporte le plus grand nombre d'attestations du gros calibre. La quantité de thylles observée sur les chênes de la double palissade est conséquente, révélant une présence importante de duramen dans les restes carbonisés de cet ensemble, ce qui est cohérent avec la présence marquée de bois de gros calibre.

La moyenne des largeurs de cernes du chêne est ici inférieure à celle mesurée au premier âge du Fer, soit 2,37 \pm $1,51 \mathrm{~mm}$; elle se rapproche de la moyenne observée à l'âge du Bronze mais les valeurs sont plus étalées et leur mode est décalé comme le montrent les histogrammes de classes (fig. 55).

Trois groupes semblent se profiler : un premier autour des largeurs 0,75 à $1,5 \mathrm{~mm}$, à croissance sous forte contrainte, un deuxième, aussi à croissance contrainte mais moindre, de 1,5 à $2,75 \mathrm{~mm}$, et bon nombre de valeurs de forte croissance au delà de $3 \mathrm{~mm}$, jusqu'à des valeurs de croissance libre.

En détaillant ces résultats par type de construction (fig. 61), il apparaît que ces différents groupes s'individualisent. Ainsi, les faibles croissances sont observées principalement au niveau de la double palissade sud (6a), tandis que les croissances moyennes sont l'apanage des chênes du talus $6 \mathrm{~b}$. Bien que les mesures aient été rares pour le bâtiment sud (6c), il ne semble pas comporter de chêne à très forte contrainte de croissance et présente au contraire des éléments à bonne voire très bonne croissance.

La moyenne des largeurs de cernes par type de construction conduit aux mêmes constatations : faible croissance pour les chênes de la double palissade et forte croissance dble palis sade sud

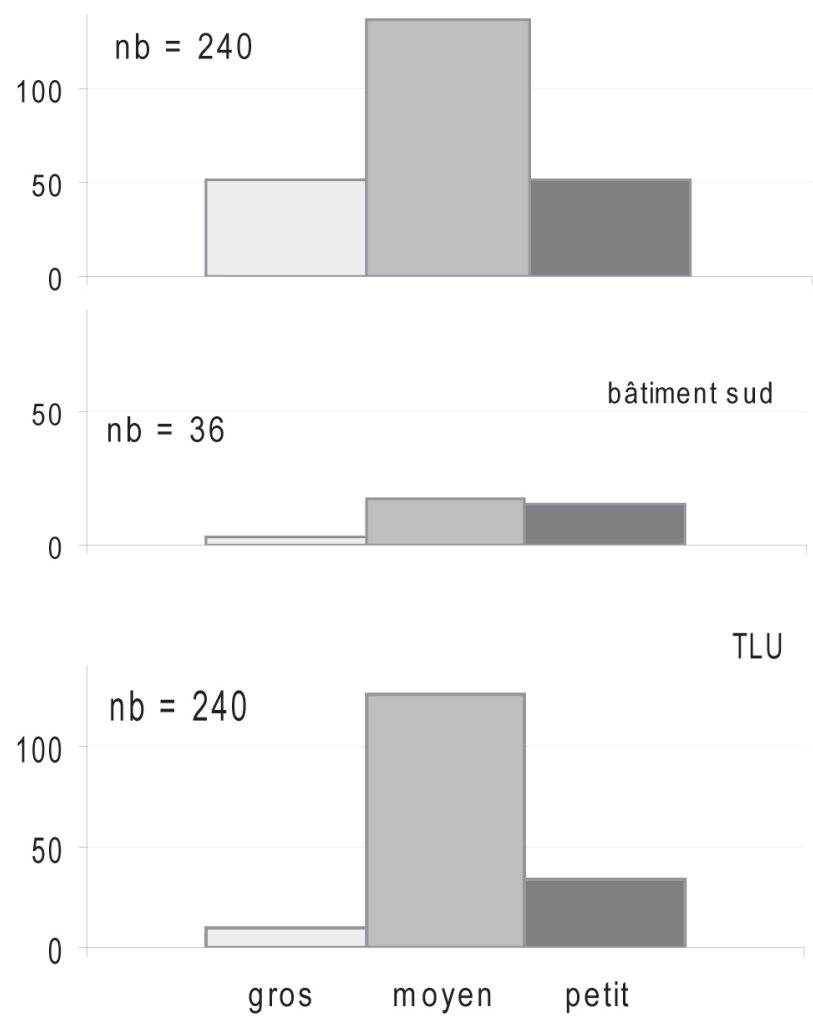

Figure 60 : Haut Moyen Âge : nombre d'observations des calibres (gros, intermédiaire, petit) et de la présence de thylles sur les charbons de chêne.

Figure 60: Early Mediaeval: observed sizes (large, middle, small) and of thylle presence on oak charcoals.

pour ceux du bâtiment sud. D'un point de vue technologique, les chênes à forte croissance, donc à cernes larges, ont une meilleure résistance mécanique. Les résultats obtenus ici reflètent vraisemblablement un choix technologique des pièces de bois en fonction des constructions, le bâtiment sud nécessitant des bois de plus forte résistance que la double palissade.

La répartition des largeurs de cernes du chêne par structure permet de faire deux constats (fig. 62). Dans le cas de la palissade 6a (TP.477-479, 481-483, 485, 544, 549) les distributions sont relativement homogènes et laissent à penser que les bois la composant ont été prélevés dans le même type de milieu, voire la même coupe. Dans l'hypothèse d'une seule pièce de bois, du moins dominante, dans chaque trou de poteau, l'étalement important des valeurs, de la classe $0,25-0,5 \mathrm{~mm}$ à la classe de plus de $6 \mathrm{~mm}$, refléterait des conditions de croissance changeantes au fil du développement de l'arbre. Les modifications du milieu pouvant 
Figure 61 : Haut Moyen Âge : histogramme des largeurs de cernes mesurées sur les charbons de chêne par type de construction. Figure 61: Early Mediaeval: rings width measured on oak charcoals, by building type.

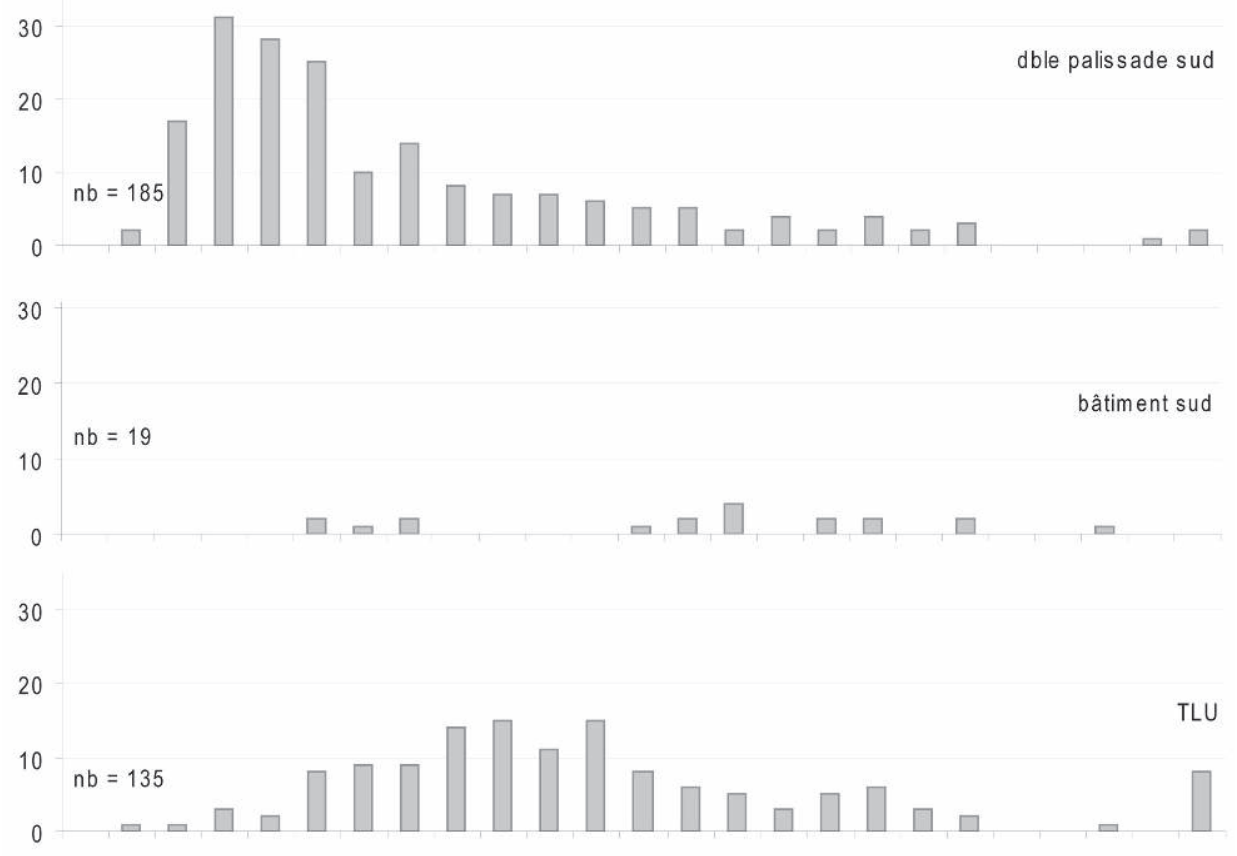

conduire à de tels écarts de croissance peuvent difficilement être d'origine naturelle et pourraient correspondre à des interventions de l'homme dans la forêt, à des coupes ou à des éclaircies.

Les bois du talus $6 \mathrm{~b}$ donnent une toute autre image. Le prélèvement TLU.3 affiche une croissance contrainte, avec des valeurs bien regroupées. Ce chêne a dû croître dans un milieu relativement stable mais peu propice à son développement, pour des raisons de densité de peuplement ou de pauvreté minérale de la station. À l'opposé, les prélèvements TLU.4 ne comportent pas de chênes à croissance très faible. Les largeurs de cernes majoritaires correspondent à des valeurs moyennes de futaie actuelle. Les valeurs plus élevées, au delà de $4 \mathrm{~mm}$, sont le reflet d'une ouverture du milieu.

Au haut Moyen Âge, les chênaies-hêtraies étaient vraisemblablement les formations forestières dominantes dans la région. Les pièces de bois utilisées à La Rochette ont été pour partie prélevées dans ce type de formation. Les observations dendrologiques sur le chêne montrent que celles-ci ont été sélectionnées en fonction des usages. La sélection s'est opérée sur leur taille : des gros calibres pour le bâtiment sud et surtout la palissade sud, et des bois de calibre plutôt intermédiaire au niveau du talus. Une préférence semble aussi se marquer quant à la croissance des arbres; en effet, des chênes aux cernes très larges, donnant des bois de meilleure résistance mécanique, ont été utilisés préférentiellement pour la construction du bâtiment sud. Ces résultats reflètent une gestion forestière raisonnée; en témoigne plus encore le grand éventail de largeurs de cernes sur un même chêne, vraisemblable marqueur des interventions humaines sur la forêt.

Comme à l'âge du Bronze, les bois ne proviennent pas de l'éperon même, mais de stations riches, comme le montre la présence du noisetier, voire très riches comme le montre la présence du frêne; elle(s) devai(en)t se situer en bas de pente et à proximité d'un cours d'eau. Le genêt/ajonc est en outre le témoin de zones ouvertes.

\section{Le SITE DE LA RochetTe DANS SON CONTEXTE ENVIRONNEMENTAL}

\section{A. Au Néolithique ancien}

Au Néolithique ancien, le paysage régional se compose de chênaies (Gaudin, 2004; Marguerie, 1992a). Le chêne est alors le taxon arborescent dominant; accompagné du tilleul, il forme des futaies régulières qui se renouvellent au fil des ouvertures du milieu, suite à des chablis ou à la sénescence et mort des semenciers. Ces futaies régulières forment des blocs de 15 ares à 15 hectares d'âges différents qui, accolés, constituent à l'échelle régionale une futaie irrégulière en mosaïque. Le sous-étage forestier dépend de la qualité des stations; les plus fraîches comportent principalement du houx, les plus riches du noisetier, et les plus sèches de petits fruitiers (Pomoïdées et Prunoïdées), le plus commun étant le poirier à feuille en cœur. 

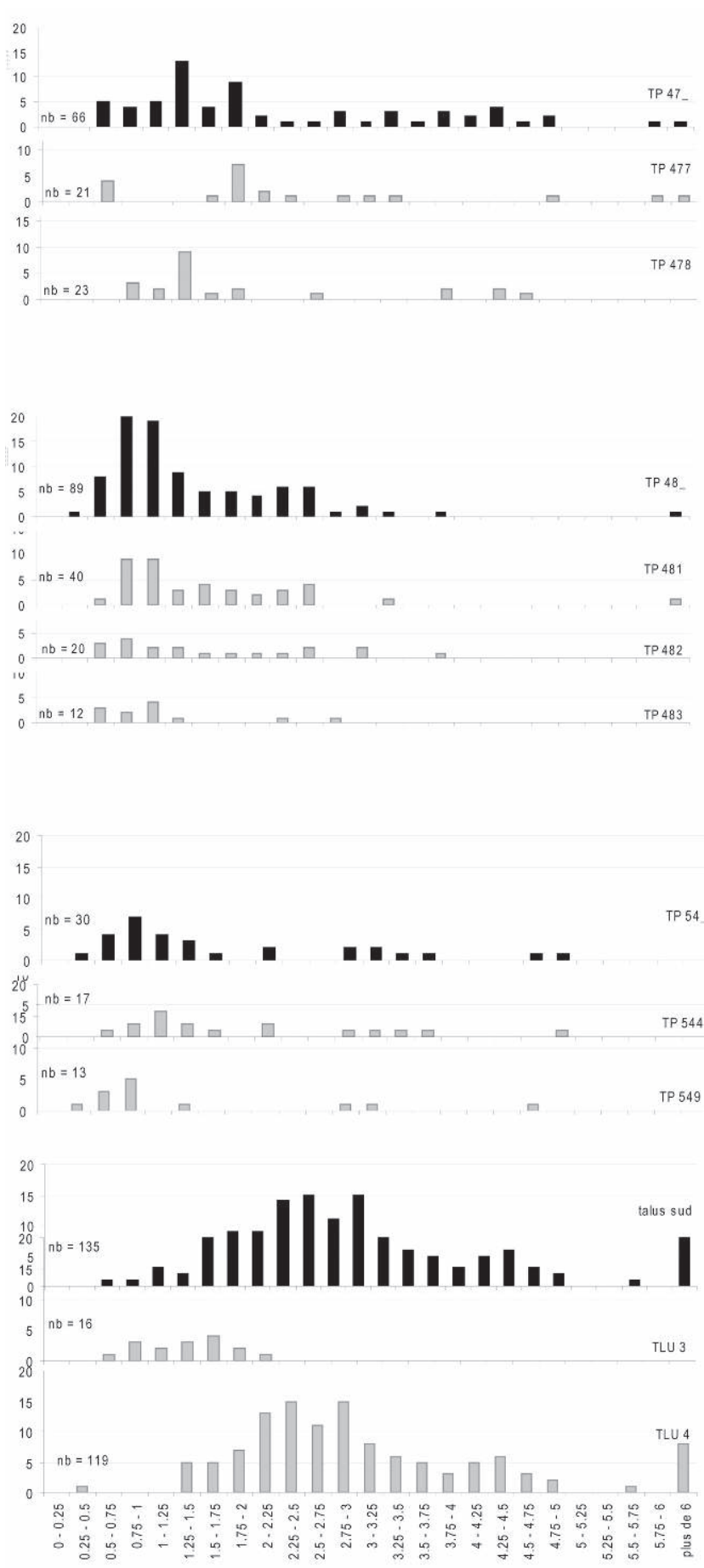

Figure 62: Haut Moyen Âge : histogrammes des largeurs de cernes mesurées sur les charbons de chêne par structures : palissade $6 \mathrm{a}$ (les trois diagrammes supérieurs)et talus $6 \mathrm{~b}$ (en bas).

Figure 62: Early Mediaeval Ages: rings width measured on oak charcoals by structures: palisade $6 a$ (the three upper diagrams) and bank 6b (below).
C'est dans ce dernier type de milieu que les bois ayant alimenté le foyer néolithique de La Rochette ont vraisemblablement été prélevés; ce pourrait d'ailleurs être sur l'éperon même. Certains charbons de chêne proviennent de bois de gros calibre, mais la plupart de calibres intermédiaire et petit. Les cernes sont étroits (moins de $1 \mathrm{~mm}$ ), confirmant la forte contrainte de croissance qu'ont connu ces chênes. Elle est vraisemblablement liée à la densité de la chênaie, accentuée par la pauvreté minérale du sol. La présence de galeries d'insectes dans ces bois et ceux de Pomoïdées indiquent que les arbres étaient sénescents voire morts au moment de la récolte.

Les sites datant de cette période et ayant fait l'objet d'une analyse anthracologique sont relativement nombreux en Bretagne et Basse-Normandie : dans le sud de la péninsule armoricaine, la Table-des-Marchands (Locmariaquer, Morbihan : Marguerie et Marcoux, 2007), Lannec-erGadouer (Erdeven, Morbihan : Gaudin et Marguerie, 2000); Kerdruelland (Belz, Morbihan : Marcoux et Marguerie, 2007); dans le nord-est d'Ille-et-Vilaine, le Haut- Mée (Saint-Étienne-en-Coglès : Marguerie, 1998); en Basse-Normandie, Le Lazzaro (Colombelles, Calvados : Marcoux et al., en préparation). Les données issues de ces études s'apparentent fortement aux résultats obtenus à La Rochette et corroborent l'interprétation proposée. Partout, le chêne à cernes étroits est le taxon dominant et il est systématiquement accompagné par les Pomoïdées. Le noisetier est récurrent, ainsi que l'érable dans une moindre mesure.

\section{B. À l'âge du Bronze}

À l'âge du Bronze, le cortège de ligneux a peu changé dans le Massif armoricain et ce sont les mêmes essences que celles caractérisant le Néolithique ancien qui ont été observées pour cette deuxième occupation de La Rochette; le taxon principal reste le chêne, accompagné du genêt/ajonc, du noisetier, et de l'érable. Cependant, les données concernant le chêne laissent entrevoir un changement dans la configuration des formations forestières; en effet, les bois sont désormais majoritairement de calibre intermédiaire (peu de pièces de gros calibre), les largeurs de cernes sont plus importantes et leur gamme plus étendue. Le site proche le plus contemporain est celui de La Basse-Bouexière (Guichen, Ille-etVilaine), daté autour de 3000 BP. Il s'agit d'un habitat dont le cortège anthracologique corrobore ces observations, à savoir le chêne dominant (moyenne des largeurs de cerne $1,96 \pm 0,56 \mathrm{~mm}$ ), accompagné de Pomoïdées et d'érable.

Régionalement, les données paléobotaniques ont enregistré une nette ouverture du milieu à partir du Néolithique final (Gaudin, 2004; Marguerie, 1992a). À Ploërmel et Néant-sur-Yvel (Morbihan), le taux de pollens d'arbres n'est plus que de $35 \%$ et $55 \%$ respectivement, les plantes 
rudérales étant très présentes sans toutefois d'attestations de cultures de céréales à Néant-sur-Yvel (Marguerie, 1992a). À Paimpont, des pollens de céréales sont enregistrés bien que la chênaie se maintienne, le chêne étant en plein essor tandis que le tilleul et le noisetier sont en recul (Oillic, com. pers.). Les résultats de l'analyse anthracologique du site de La Hersonnais à Pléchâtel (Ille-et-Vilaine) reflètent ces changements (Tinévez, 2004); le cortège des ligneux y est diversifié (quinze taxons différents ont été observés) et la croissance des chênes est au delà de $1 \mathrm{~mm}$ par an $(1.33 \pm$ $0,49 \mathrm{~mm}, 1.26 \pm 0,46 \mathrm{~mm}$, et $1.77 \pm 0,83 \mathrm{~mm}$ ). Toutefois, les chênes sont essentiellement de gros calibre et les largeurs de cernes sont bien regroupées autour de la moyenne, révélant la persistance de belles chênaies matures.

Depuis ces premiers développements de l'agropastoralisme qui ont fortement marqué le paysage régional, les défrichements se sont poursuivis, bien qu'ils semblent demeurer d'échelle réduite. Et un ralentissement, voire une reconquête des taxons forestiers est probable au moment de la péjoration climatique de l'âge du Bronze moyen au Bronze final, entre 1550 et 1200/1050 BC (Richard et al., 2007). C'est donc dans ce contexte de paysage en mosaïque que s'inscrit la deuxième occupation du site de La Rochette.

\section{C. Au premier âge du Fer}

Le contexte environnemental de cette troisième occupation est probablement peu différent du précédent, si ce n'est des zones défrichées plus nombreuses, une présence du hêtre de plus en plus soutenue aux portes du Massif armoricain et l'apparition de nouvelles cultures telles que le sarrasin, attesté vers 2726 BP à Inguiniel (Morbihan) (Marguerie, 2001). Les charbons mis au jour sur le site de Beaumont, un habitat de la Tène finale à Saint-Laurent-sur-Oust, Morbihan (Marguerie, 1996) semblent être le reflet d'un tel milieu fortement anthropisé, avec le genêt/ajonc et le noisetier comme taxons prédominants, accompagnés de chênes de calibres intermédiaire et petit, et de Pomoïdées.

Les charbons de La Rochette sont alors majoritairement du chêne à forte croissance, accompagné du genêt/ajonc. Plusieurs des échantillons présentent des fibres torses et des zones poreuses mal définies (souches? racines?) et l'interprétation des résultats du foyer étudié (fo.09) apparaît hasardeuse. En effet, il peut tout à fait s'agir d'un seul et même arbre ayant poussé au milieu d'un champ et ayant été victime d'un chablis.

\section{D. Au haut Moyen Âge}

Le contexte environnemental du haut Moyen Âge est très différent de celui des périodes précédentes. D’une part,
La Tène et le Haut-Empire ont vu les surfaces forestières armoricaines se réduire sérieusement et, parallèlement, la lande à calluna se développer (Gaudin, 2004; Marguerie, 1992a). Les besoins en surfaces cultivées et en pâtures sont de plus en plus grands, et surtout ceux en combustibles pour les diverses activités artisanales. Des futaies évoluent peu à peu en taillis; malgré tout, des forêts (ou au minimum des bois) comportant de beaux fûts subsistent comme le montrent les charbons de chênes de gros calibre et de croissance contrainte (1-2 mm/an) des sites gallo-romains d'Ille-etVilaine : Beziers à Bédée (Marguerie et Renaudin, 1997), Chartres-de-Bretagne (Marguerie, 1993), La Chapelle à Cesson-Sévigné (Marguerie, 1997). Le paysage du Massif armoricain a aussi été transformé entre-temps par le déploiement du hêtre, les chênaies se transformant en chênaieshêtraies comme rappelé plus haut (Giesecke et al., 2007; Davis et al., 2003).

Le Bas-Empire et l'époque mérovingienne ont été des périodes tumultueuses tant du point de vue politique qu'économique, démographique et climatique. Il est probable que ces événements ont eu un effet sur l'environnement mais les études sur ce sujet en sont encore à leur balbutiement. La période carolingienne est, quant à elle, marquée par un retour à la stabilité et un essor important des cultures, entre autres dans le Coglais (Marguerie, 2002) et dans le centre-est du Morbihan (Visset, 1989). Au moment de la quatrième occupation du site de La Rochette, au haut Moyen Âge, le paysage alentour est ainsi probablement agraire, du moins en grande partie.

Les données anthracologiques montrent néanmoins que des forêts, au minimum des bois, composés de beaux fûts de chêne et de hêtre, subsistent toujours. Ces ressources forestières ont été exploitées de façon raisonnée à La Rochette : les chênes à croissance rapide, fournissant une meilleure résistance mécanique, ont été réservés pour la construction du bâtiment sud, les chênes de gros calibre ont servi à la construction de la palissade sud et ceux de calibre intermédiaire se retrouvent au niveau du talus. La grande gamme des largeurs de cernes au sein d'un même trou de poteau, ne comportant a priori - ou du moins majoritairement qu'un seul bois, laisse entrevoir des interventions régulières de l'homme dans les forêts.

Les analyses anthracologiques effectuées au Camp de Péran dans les Côtes-d'Armor (Marguerie, 1992a) s'apparentent étroitement aux résultats obtenus à La Rochette et viennent renseigner l'utilisation possible des différents taxons dans les constructions. Au Camp de Péran, les chênes et les hêtres de gros calibre ont été utilisés pour la construction de bâtiments, tandis que le frêne, l'aulne, le noisetier, le bouleau, le genêt et une Pomoïdée ont servi à l'élaboration de clayonnages. À La Rochette, ce dernier usage a probable- 
ment été celui du genêt/ajonc (bâtiment sud), du noisetier et du saule (palissade).

Le site de la Cocherais (Tinténiac, Ille-et-Vilaine) comporte aussi des chênes de gros calibre, ainsi que du hêtre (Marguerie, 1992b). Des mesures de largeurs de cernes sur les chênes y ont montré que les bois de construction sont mieux classés autour de la moyenne tandis que les bois utilisés dans les tranchées-foyers de séchage/grillage sont répartis sur un grand éventail de largeurs. En cela, l'histogramme des tranchées-foyers s'apparente à celui des bois du talus de La Rochette. À la Cocherais, la moyenne des largeurs de cernes est élevée au sein des constructions $(3,22 \pm 0,39 \mathrm{~mm})$; elle est de 3,42 $\pm 1,26 \mathrm{~mm}$ pour le bâtiment sud de La Rochette.

\section{ConClusions SUR L'ÉTUde ANTHRACOLOGIQUE}

La Rochette sera un site de référence pour les études anthracologiques à venir sur le Massif armoricain. Les occupations successives du Néolithique ancien, de l'âge du Bronze, du premier âge du Fer et du haut Moyen Âge ont permis d'y observer l'évolution des bois et de leur usage dans le temps sur un même site. L'analyse des charbons de bois, bien qu'ils proviennent tous de structures, a permis de faire une première approche des lieux de prélèvement et de l'environnement autour du site au moment des différentes occupations. Des études paléobotaniques contemporaines sont venues affiner les interprétations.
La valeur de référence de ce site est liée à la chronologie précise des différentes occupations. En effet, chacune d'elle a bénéficié d'une ou plusieurs dates radiocarbone; ce sont ces repères chronologiques qui permettent de mettre en contexte les résultats et d'en tirer le maximum d'informations.

Une quantité importante de charbons de La Rochette est conservée au laboratoire de l'UMR 6566 de Rennes. Il est à souhaiter que des analyses complémentaires soient effectuées, entre autre pour affiner les données sur les calibres et sur les largeurs de cernes.

\section{ConClusion GÉNÉrale (J.-Y. T.)}

Une évolution de l'espace enclos est nettement perceptible entre les trois phases principales d'implantation sur le promontoire de La Rochette et une restitution des aménagements successifs peut être proposée (fig. 63 et 64). Cela se traduit par une réduction progressive de l'emprise protégée entre les occupations de l'âge du Bronze, du premier âge du Fer et du haut Moyen Âge. À l'âge du Bronze, le système de barrage édifié à plus de $170 \mathrm{~m}$ de la pointe du promontoire assure la protection d'une surface de 1,7 ha.

La première proposition de restitution (fig. 63-1 et 64 A) ne représente que les structures de l'âge du Bronze attestées par la fouille : la palissade nord et ses deux entrées, le large fossé segmenté doublé d'un rempart interne, la série de bâtiments en arc de cercle. Une possible palissade surmontant le rempart n'est pas représentée en l'absence de toute trace
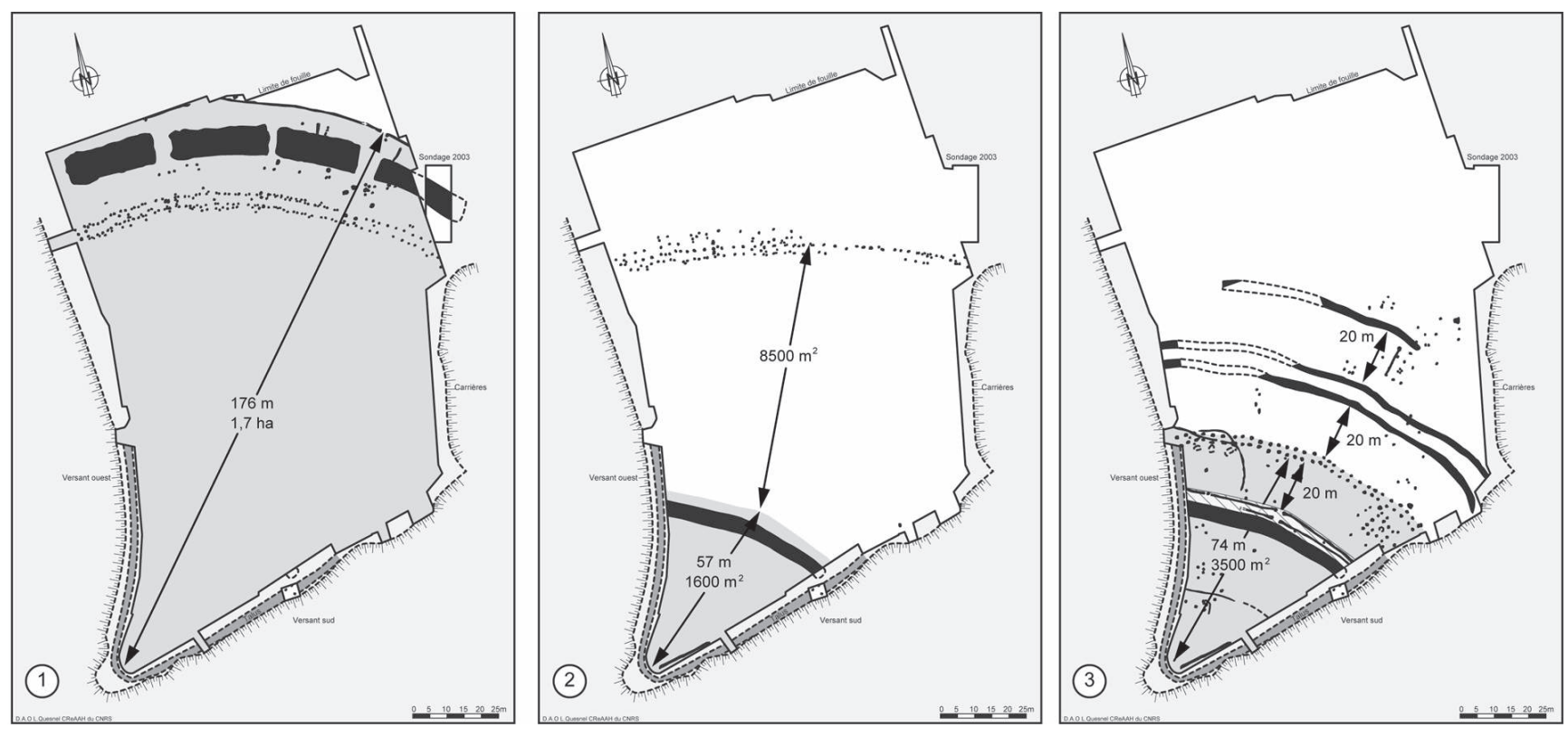

Figure 63 : Évolution de l'espace occupé et surfaces d'emprise : 1, âge du Bronze; 2, âge du Fer; 3, haut Moyen Âge. Figure 63: Evolution of the occupied area: Bronze Age (1), Iron Age (2) and early Mediaeval (3). 

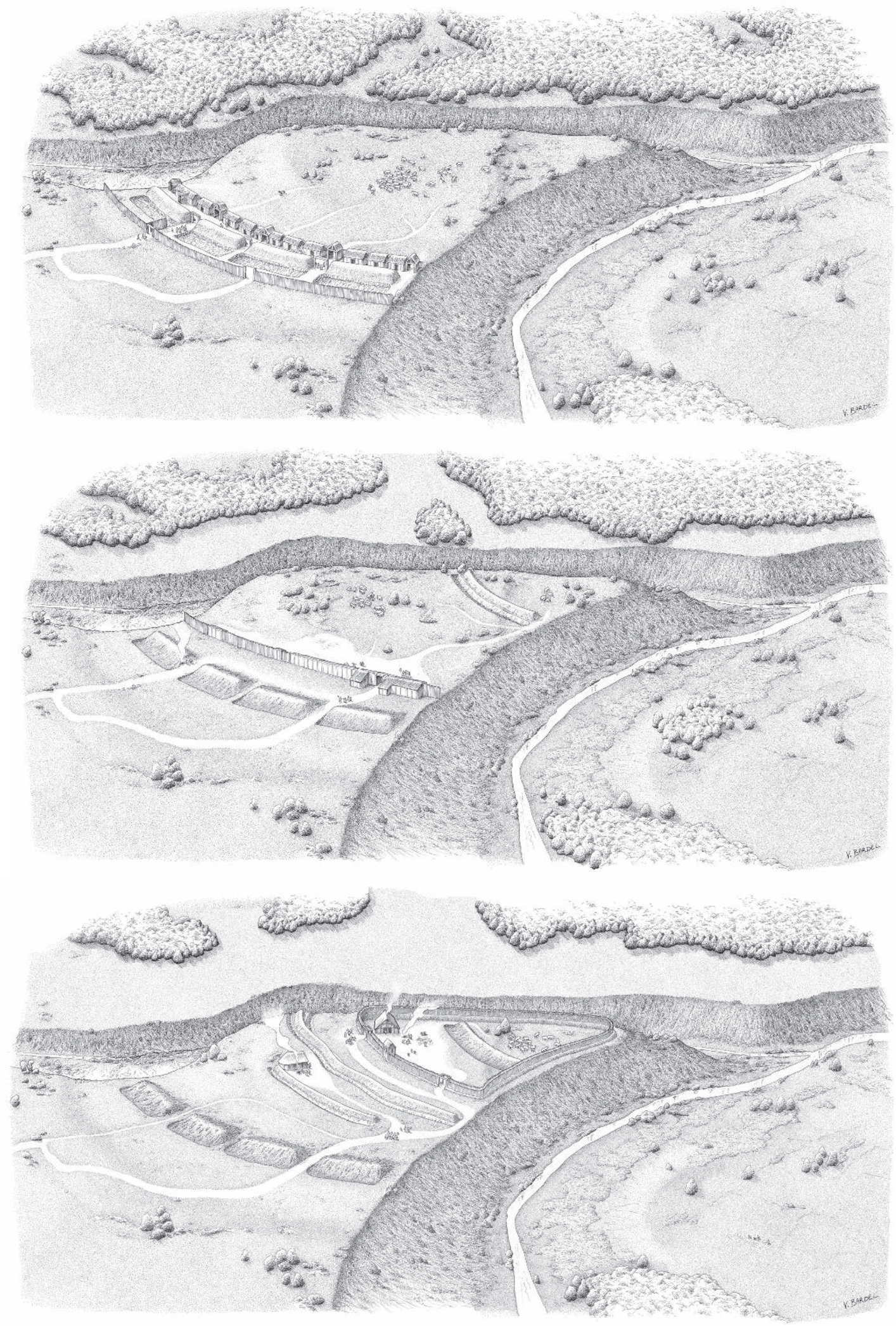

Figure 64 : Propositions de restitution des occupations du promontoire : de haut en bas, âge du Bronze, premier âge du Fer et haut Moyen Âge (dessins Véronique Bardel).

Figure 64: Hypothetical reconstructions of the promontory occupations: from top to bottom, Bronze Age, first Iron Age and early Mediaeval (Véronique Bardel drawings). 
archéologique; en revanche, les portes figurées dans le rempart ont bien été localisées à la fouille.

Au premier âge du Fer (fig. 63-2 et 64 B), la nature de l'occupation est plus incertaine. Une première palissade sur laquelle s'adossent de petits bâtiment délimite une aire d'un hectare environ, nettement inférieure à celle de l'occupation précédente. Le nouveau fossé de barrage, creusé à $57 \mathrm{~m}$ de la pointe, ne ceinture que $1600 \mathrm{~m}^{2}$ de surface. La restitution met en évidence la présence résiduelle du grand fossé, en partie comblé et en bordure sud-est duquel s'installe un foyer. De petits ensembles de trous de poteau, sans plan précis et disséminés dans la partie centrale du promontoire n'ont pas été représentés, faute d'attribution chronoculturelle attestée.

Les importants aménagements du haut Moyen Âge resserrent l'occupation vers le sud du promontoire (fig. 63-3 et $64 \mathrm{C}$ ), avec une emprise de $3500 \mathrm{~m}^{2}$ pour l'enceinte triangulaire principale qui barre l'accès à $75 \mathrm{~m}$ de la pointe. Pour la restitution, le parti est pris d'associer les talus et fossés avancés en raison du parfait parallélisme des tracés et l'écartement régulier de $20 \mathrm{~m}$ entre les lignes de barrage. De même, l'hypothèse de la présence des deux portes et d'une tour centrale dans la palissade, justifiée par l'écartement particulier des poteaux à ces endroits précis, est figurée sur le dessin de restitution. L'impact estompé des aménagements antérieurs, fossé interrompu au nord et fossé de l'âge du Fer au sud, est toujours visible sur le dessin.

Au démarrage de cette opération, la problématique de recherche était centrée sur le fossé de barrage segmenté, avec l'hypothèse d'une attribution chronologique à la fin de la Préhistoire par comparaison avec de nombreux sites des régions voisines de la Bretagne, la présence de fossés continus vers la pointe du promontoire laissant présager l'existence d'occupations diachroniques. A l'issue de quatre années d'études de terrain, les résultats modifient sensiblement les hypothèses de départ, sur la chronologie des occupations notamment, mais n'enlèvent rien à l'intérêt scientifique de la fouille. En effet, l'étude quasi exhaustive du promontoire triangulaire de La Rochette a mis en évidence une succession d'occupations dont les vestiges conservés sont inédits sur le plan régional.

Si les traces du Néolithique ancien restent très ténues, elles constituent néanmoins un nouveau jalon sur l'avancée dans la péninsule des premiers agriculteurs au début du Ve millénaire.

En revanche, l'âge du Bronze est marqué par une forte implantation très structurée, remarquable par sa régularité et la parfaite adéquation des aménagements au relief existant. Un système complexe de barrages successifs - palissade, fossé segmenté, rempart - protège un ensemble d'édifices dont la fonction domestique semble la plus probable, par compa- raison avec plusieurs sites similaires connus dans l'ouest de la France et le Bassin Parisien. Ainsi, le site de La Rochette confirme une tendance reconnue sur l'ensemble du territoire national et caractérisée par la multiplication des sites fortifiés à l'âge du Bronze, en relation avec une occupation $\mathrm{du}$ territoire de plus en plus dense et conflictuelle et une hiérarchisation sociale de plus en plus poussée. Le statut particulier de cet habitat fortifié peut être mis en parallèle avec celui du site de Boulancourt dans le Bassin parisien, sur lequel une économie agropastorale de type aristocratique a été mise en évidence (Balasescu et al., 2008).

Au cours du premier âge du Fer, l'occupation du promontoire reste plus lacunaire et difficile à interpréter, bien que la réalisation du fossé sud ait demandé un investissement non négligeable. L'absence d'habitat clairement défini associé aux systèmes de barrage - palissade puis fossé-rempart - laisse une impression de site abandonné en cours d'élaboration, même si l'on peut évoquer la possibilité de constructions sans fondations profondes et mal conservées.

Les travaux de terrassement conséquents réalisés durant ces deux implantations vont marquer durablement le relief du lieu, mais il faudra attendre plusieurs siècles, jusqu'au haut Moyen Âge, à la transition entre les époques mérovingienne et carolingienne, avant de voir le site réinvesti sur un modèle et avec un statut défensif similaires. La destruction violente et totale par incendie de la nouvelle enceinte en bois et de l'édifice qu'elle protégeait pose la question de la durée de son utilisation dans un contexte historique instable.

Ces trois occupations principales ont en commun de refléter une forte volonté de protection mettant en œuvre des moyens importants pour une durée d'utilisation, qui à chaque fois semble courte en l'absence de réaménagements multiples. En tant que site-refuge à certaines périodes, l'éperon barré est à mettre en relation avec son contexte archéologique et environnemental. Sa position légèrement à l'écart d'un réseau viaire important et non loin d'un franchissement de l'Yvel est à prendre en compte, probablement dès la Protohistoire.

Une analyse anthracologique globale, menée sur des charbons de bois abondants et représentatifs des périodes concernées, insère le site dans l'évolution environnementale de la péninsule armoricaine du Néolithique aux périodes historiques, avec une tendance progressive à l'ouverture du milieu par défrichements et pratiques agropastorales. D'abord limité et discontinu au cours de la Protohistoire, cet impact devient plus étendu et pérenne à partir de la période antique. Les taxons datés du haut Moyen Âge font apparaître de nouvelles essences - le hêtre notamment, venant concurrencer le chêne comme bois d'œuvre - et le frêne, peut être lié à l'élevage en tant que fourrage. 
Les aménagements diachroniques révélés sur le promontoire de La Rochette incitent à être prudent sur l'interprétation de vestiges qui, à première vue, semblent contemporains et complémentaires par leur proximité et leur parallélisme, alors qu'en réalité, ils résultent d'une longue succession d'occupations motivées par une topographie particulière.

\section{Remerciements}

C'est avec un grand plaisir que j'adresse mes remerciements à toutes les personnes qui ont contribué à la mise en oeuvre et à la réalisation de cette opération. L'autorisation de sondage má été accordée par le ministère de la Culture et de la Communication grâce à la diligence de Stéphane Deschamps, conservateur régional de l'archéologie, de Guirec Querré, directeur de l'UMR 6566 et de Christine Jablonski, conservateur du Patrimoine en charge du département du Morbihan. Je remercie vivement les propriétaires du site, $M$. et $M^{m e} B l o t, M$. et $M^{m e}$ Piéderrière, pour leur autorisation et leur amabilité. Le financement a été assuré conjointement par la sous-direction de l'Archéologie et le conseil général du Morbihan. La gestion des financements par le CNRS a été menée à bien grâce à la vigilance et l'investissement de Michèle Tostivint au laboratoire d'Anthropologie de Rennes.

Inventeur du site, Maurice Gautier a mis en permanence à notre disposition les fructueux résultats de ses missions aériennes qui participent pleinement à nos recherches. $\grave{A}$ travers son accueil chaleureux, Gilles Montgobert a contribué à la bonne intégration de notre équipe dans la commune et nous a fait partager ses connaissances archéologiques locales. Les municipalités de Mauron et de Saint-Brieuc-de-Mauron ont exprimé leur intérêt pour nos recherches lors de visites du chantier. La municipalité de Mauron a contribué à la préparation de la présentation du site au public lors de la Journée du Patrimoine du 18 septembre 2005.

Le relevé topographique du site a été réalisé dans le cadre d'un stage par l'Institut Universitaire de Technologie - Génie Civil de Rennes, sous la direction de Louis Martel et Thierry Lorho, géomaticien au SRA de Bretagne, a assuré le calage du carroyage par système GPS. Au sein de l'UMR 6566, Laurent Quesnel, dessinateur infographe au laboratoire $d u C N R S$, a réalisé avec efficacité et compétence les travaux de DAO des illustrations graphiques des rapports et de la publication. Francis Bertin en a assuré la mise en pages. Nancy Marcoux, avec la collaboration de Dominique Marguerie, a pris en charge l'étude anthracologique des nombreux échantillons de charbons de bois et Vincent Bernard, l'analyse dendrologique de certains d'entre eux. L'étude du macro-outillage lithique a été menée par Klet Donnart. Véronique Bardel a dessiné les propositions de restitutions.

Les multiples datations par le radiocarbone ont été réalisées successivement au Centre des faibles Radioactivités de Gif-surYvette sous la direction de Michel Fontugne, au Centrum voor
Isotopen Onderzoek de Groningen sous la direction du Prof. J. van der Plicht et au Centre de Datation par le Radiocarbone de Lyon sous la direction de Christine Oberlin.

Les vestiges mis au jour concernant plusieurs périodes, les échanges d'informations ont été fructueux avec mes collègues archéologues et historiens; je remercie notamment Anne Villard, Yves Ménez, Anne-Françoise Cherel, Laurent Beuchet, Stéphane Blanchet, Philippe Lanoë et Alain Provost. José Gomez de Soto et Charles-Tanguy Le Roux ont assuré une correction détaillée du manuscrit.

Ma reconnaissance va tout particulièrement aux fouilleurs bénévoles qui ont auvré à cette opération avec efficacité et enthousiasme: Juliette Abolivier, Sylvie Bacquelaine, Aurélia Becuwe, Laétitia Bertrand, Damien Bonniol, Romaric Boquart, Sophie Borg, Christine Boujot, Yann-Steven Cadic, Stéphanie Chalmel, Cédric Chatellier, Céline Choquenet, Jérôme Couderc, Solène Danet, Alice Dinechin, Klet Donnart, Claire Dupin, Victor Faucheux, Bertrand Francqueville, Camille Gandonnière, Florian Gasco, Claire Gayot, Gwénaële Gourlay, Stéphanie Hedda, Mathieu Hillairet, Cédric Holleville, Natacha Jamet, Nolwenn Jarnoux, Fabien Jonquois, Géraldine Jouquand, Aurore Lambert, Erwan Le Balch, Rémi LeblancMessager, Elodie Lecher, Laurence Le Clézio, Nolwenn Le Faou, Sterenn Le Maguer, Kristell Lemoine, Romaric Le Montagner, Frédéric Lévêque, Karine Loyer, Alexandre Lucquin, Lorraine Manceau, Gilles Ménard, Céline Merrer, Myriam Michel, Stéphanie Moutaque, Elodie Pigeon, Anne Pondaven, Victor Richert, Adeline Quenouillère, Nolwenn Quinio, Jean-Philippe Rolin, Isabelle Rondeau-Baron, Julien Rouaud, Maîa Saur, David Shallcross, François-Xavier Simon, Elin Söderman, Benoît Thierry, Ewen Tinévez et Lorie Tremblay.

\section{Bibliographie}

Balasescu, A., Simonin, D. et Vigne, J.-D., 2008 - La faune du Bronze final IIIb du site fortifié de Boulancourt « Le Châtelet » (Seine-et-Marne), Bulletin de la Société préhistorique française, 105 (n²), p. 371-406.

Baneat, P., 1928 - Le Département d'Ille-et-Vilaine. Histoire - Archéologie - Monuments (Tome II), Rennes, Librairie moderne, Rennes (et rééd. 1994, Mayenne, Éditions régionales de l'Ouest) $534 \mathrm{p}$.

Blanchet, J.-C., 1984 - Les premiers métallurgistes en Picardie et dans le nord de la France, Paris, Société préhistorique française (Mémoires, 17), 608 p., 251 fig.

Blanchet, J.-C. et Talon, M., 1987 - L'éperon barré du « Camp César " à Catenoy (Oise) à l'âge du Bronze final. Premiers résultats, in BlANCHET, J.-C. (dir.), Les relations entre le continent et les îles Britanniques à l'âge du Bronze, Actes du colloque 
de Lille dans le cadre du $22^{e}$ congrès préhistorique de France, Amiens, Revue archéologique de Picardie/Société préhistorique française, p. 189-210.

Blanchet, S., Cherel, A.-F., Fromont, N., Forre, P., Hamon, C. et Hamon, G., 2007 - Rapport final d'opération de Betton "Pluvignon" (Ille-et-Vilaine), vol. 1, le Néolithique ancien, Rennes, Inrap Grand-Ouest, 280 p.

Brown, N., 1996 - The Archaeology of Essex, c. 1500-500 BC, in Bedwin, O. (ed.), The Archaeology of Essex: Proceedings of the 1993 Writtle conference. Chelmsford, Essex County Council, p. 26-37.

Brown, N., 2001 - The Late Bronze Age enclosure at Sprinfield Lyons in its Landscape context. Essex Archaeology and History, 32, p 92-101.

Cassen, S., Audren, C., Hingant, S., Lannuzel, G. et Marchand, G., 1998 - L'habitat Villeneuve-Saint-Germain du Haut-Mée (Saint-Etienne-en-Coglès, Ille-et-Vilaine), Bulletin de la Société préhistorique française, 95 ( $\left.{ }^{\circ} 1\right)$, p. 41-75.

Chapelot, J. et Fossier, R., 1980 - Le village et la maison au Moyen Âge. Paris, Hachette littérature, 357 p.

Catteddu, I. (dir.), 2001 - Les habitats carolingiens de Montours et La Chapelle-Saint-Aubert (Ille-et-Vilaine), Paris, Maison des Sciences de l'Homme (DAF, 89), 235p.

Davis, B. A. S., Brewer, S. et Stevenson, A. C., 2003 - The temperature of Europe during the Holocene reconstructed from pollen data. Quaternary Science Reviews, 22, p. 1701-1716.

Delrieu, F., 2007 - Igé, Le Crochemélier (Orne), Bilan Scientifique de Basse-Normandie, Caen, Service régional de l'Archéologie, p. 111-112.

Donnart, K., 2007 - Première approche diachronique du macrooutillage dans le Massif armoricain : du Néolithique moyen au début de l'âge du Bronze (mémoire de master 2), Rennes, université de Rennes 1, 27 p.

Donnart, K., 2010 - L'analyse des Unités techno-fonctionnelles appliquée à l'étude du macro-outillage néolithique, L'Anthropologie, 114, (n²) p. 179-198.

DonnarT, K., à paraître - Le matériel de mouture de l'habitat campaniforme/Bronze ancien de Beg-ar-Loued (île Molène, Finistère) : étude préliminaire, Evolution typologique et technique des meules du Néolithique à l'an mil sur le territoire français (actes de la table ronde de Saint-Julien-sur-Garonne, 2009), Bordeaux, supplément à Aquitania.

Gaiffe, O., Laporte, L., Rouzeau, M.-H. et Rouzeau, M. (avec la collaboration de Bodeur, Y., Gruet, Y., Maggy, C. et Pirault, L.), 1995 - Le camp protohistorique de Penchâteau au Pouliguen (Loire-Atlantique), Revue archéologique de l'Ouest, 12, p. 117-137.

Galliou, P., Dare, S., Naas, P., Gautier, M. et Triste, A., 2009 - Le Morbihan 56, Carte Archéologique de la Gaule, Paris, Académie des Inscriptions et Belles Lettres, 445 p.
GAUDIN, L., 2004 - Les transformations spatio-temporelles de la végétation du nord-ouest de la France depuis la fin de la dernière glaciation. Reconstitutions paléo-paysagères (thèse de doctorat), Rennes, université de Rennes 1 (2 volumes), 763 p.

Gaudin, L. et Marguerie, D., 2000 - Étude anthracologique du tertre mégalithique de Lannec-er-Gadouer. Éléments paléoenvironnementaux et palethnographiques, in CASsen, S. (dir.), Eléments d'architecture. Constructions et reconstructions dans le Néolithique morbihannais, propositions pour une lecture symbolique. Chauvigny (France), Association de Publications chauvinoisesEd. Chauvinoises, p. 139-148.

GaUtier, M., 1993 - Les révélations archéologiques des «providentielles » missions de l'IGN des 16 et 17 juin 1976, en Pays Porhoët, Journée préhistorique et protohistorique de Bretagne (résumés des communications), Rennes. UMR 6566, p. 60-62.

Gautier, M., 1996 - Les parcellaires antiques du Porhoët et de la vallée de l'Yvel, in Chouquer, G. (dir.), Les formes du paysage, t. 1 - Études sur les parcellaires, Paris, Errance, p. 49-56.

Gautier, M., 2002 - Pour une nouvelle géographie antique des Pays de Brocéliande, in Walter, Ph. (éd.), Brocéliande ou le Génie du lieu, Grenoble, Presses universitaires de Grenoble, p. 30-48.

Giesecke, T., Hickler, T., Kunkel, T., Sykes, M. T. et Bradshaw, R. H. W., 2007 - Towards an understanding of the Holocene distribution of Fagus sylvatica L., Journal of biogeography, 34, p. 118-131.

Giot, P.-R., 1981 - En marge de l'archéologie du paysage. La date des premiers retranchements et des fortifications, Bulletin de la Société archéologique du Finistère, 109, p. 75-81.

Gouezin, P., 1994 - Les mégalithes du Morbihan intérieur, des Landes de Lanvaux au nord du département. Rennes, Institut culturel de Bretagne/Université de Rennes 1 (coll. Patrimoine archéologique de Bretagne) 127 p.

Guigon, P., 1993 - L'Architecture pré-romane en Bretagne. Le premier art roman. Rennes, Institut culturel de Bretagne/ Université de Rennes 1/Centre régional d'archéologie d'Alet, coll. "Patrimoine archéologique de Bretagne », 55 p.

Guigon, P., 1997 - Les fortifications du haut Moyen Âge en Bretagne. Rennes, Institut culturel de Bretagne/Université de Rennes 1 (coll. Patrimoine archéologique de Bretagne), 106 p.

Hamon, N.-G., 2001 - Sondages de l'enceinte du Val Aubin à Lamballe (Côtes-d'Armor), rapport de sondages d'évaluation, Rennes, SRA de Bretagne, 25p.

Le Bihan, J.-P. et Villard, J.-F., 2010 - Archéologie d'une île à la pointe de l'Europe: Ouessant, t. 2 : L'habitat de Mez-Notariou, des origines à l'âge du Bronze, in LE BiHAN, J.-P. (dir), Quimper, Centre de Recherche archéologique du Finistère. 588 p.

Le Boulanger, F. et Provost, A., 1992 - Un « village " carolingien sur la déviation de la RN 137 à La Cocherais en Tinténiac (Ille-et-Vilaine). Les Dossiers du CeRAA, 20, p. 87-117. 
LECORNEC, J., 1973 - Le site à enclos de Kerlande à Brandivy (Morbihan). Annales de Bretagne, 80 (n 1, p. 61-70.

LE Goffic, M. , 2009 - Le Souc'h a parlé. Journée du "CReAAH » Archéologie, Archéosciences, Histoire, Rennes 28 mars 2009 (résumés des communications), Rennes, UMR 6566, p. 35-39.

Leroi-Gourhan, A., 1971 - Évolution et techniques, t. 1 : L’homme et la matière, Paris, Albin Michel, 348 p. (1 $1^{\text {re }}$ éd. 1943).

LE Roux, C.-T., 1999 - L'outillage de pierre polie en métadolérite du type A. Les ateliers de Plussulien (Côtes-d'Armor): production et diffusion au Néolithique dans la France de l'ouest et au delà, Rennes, Travaux du laboratoire Anthropologie, Préhistoire et Quaternaire armoricains, $244 \mathrm{p}$.

Leroux, G., 1992 - Découvertes de structures d'habitat néolithique dans le bassin oriental de la Vilaine : l'apport de la prospection aérienne dans le sud-est de l'Ille-et-Vilaine. Revue archéologique de l'Ouest, suppl. n 5 (Actes du 17e colloque interrégional sur le Néolithique, Vannes, 1990), p. 79-83.

Leroux, G., Le Boulanger, F. et Blanchet, S., 1998 - Les occupations anciennes des rives de la Vilaine à Vieuxville-Beaurade (Rennes, Ille-et-Vilaine), de la Préhistoire à la fin du Moyen Âge. Revue archéologique de l'Ouest, 15, p. 173-199.

Marcoux, N. et Marguerie, D., 2007 - Étude anthracologique : Kerdruelland (Belz, Morbihan) (Rapport d'analyses), Rennes, UMR 6566, Université de Rennes 1, 25 p.

Marcoux, N., Marguerie, D. et Aoustin, D, en préparation Le site du Lazzaro (Colombelles, Calvados) : étude anthracologique, in Billard, C. et Bostyn, F. (dir.), Premiers paysans de la Plaine de Caen. L'habitat du Néolithique ancien de Colombelles "Le Lazzaro" (Calvados).

MArE, E., 2003 - Malleville-sur-le-Bec, A 28 Nord (Eure), Buisson du Roui. Bilan scientifique régional de Haute-Normandie 2003, Rouen, Service régional de l'Archéologie. p. 54-55.

Mare, E, 2005 - Le village de Malville-sur-le-Bec (Eure), in Marcigny, C. et al. (dir.) - La Normandie à l'aube de l'histoire, les découvertes archéologiques de l'âge du Bronze, 2300-800 av. J.-C.,Paris, Somogy, p. 52-53.

Marguerie, D., 1992a - Évolution de la végétation sous l'impact humain en Armorique, du Néolithique aux périodes historiques. Rennes, Université de Rennes 1 - UPR 403 (Travaux du Laboratoire d'Anthropologie, $\mathrm{n}^{\circ} 40$ ), $313 \mathrm{p}$.

Marguerie, D., 1992b - Le village carolingien de la Cocherais (Tinténiac, Ille-et-Vilaine), rapport d'étude anthracologique. Rennes, UMR 6566.

MarguerIE, D., 1993 - Le site artisanal de Chartres-de-Bretagne, rapport d'étude anthracologique. Rennes, UMR 6566.

Marguerie, D., 1996 - Le site de Beaumont (Saint-Laurent-surOust, Morbihan), rapport d'étude anthracologique, Rennes, UMR 6566.

Marguerie, D., 1997 - La Chapelle (Cesson-Sévigné, Ille-etVilaine), rapport d'étude anthracologique, Rennes, UMR 6566.
Marguerie, D., 1998 - Le Haut-Mée (Saint-Etienne-en-Coglès), rapport d'étude anthracologique, Rennes, UMR 6566.

Marguerie, D., 2001 - Interactions homme-milieu naturel en Morbihan intérieur : apport des études polliniques menées dans les zones humides. Mémoires de la Société d'Histoire et d'Archéologie de Bretagne, 79, p. 379-400.

Marguerie, D., 2002 - Le Teilleul (Montours, Ille-et-Vilaine), rapport d'étude paléoenvironnementale, Rennes, UMR 6566, 47 p.

Marguerie, D. et Hunot, J.-Y., 2007 - Charcoal analysis and dendrology : data from archaeological sites in Western France. Journal of Archaeological Science, 34, p. 1417-1433.

Marguerie, D. et Marcoux, N., 2007 - Etude anthracologique de la Table des Marchands, rapport d'analyses, Rennes, UMR 6566, CNRS et Université de Rennes 1, 30 p.

Marguerie, D. et Renaudin, S., 1997 - Beziers (Bédée, Illeet-Vilaine), rapport d'étude anthracologique, Rennes, UMR 6566.

Menez, Y. et Batt, M.,1988 - L'habitat du haut Moyen Âge de Creac'h-Gwen à Quimper (Finistère). Revue archéologique de l'Ouest, 5, p.123-140.

Menez, Y., (avec la coll. de Giot, P.-R., Laubenheimer, F., LE Goff, E. et Vendries, C.) 1999 - Les sculptures gauloises de Paule (Côtes-d'Armor), Gallia, 56, p. 357-414.

Nicolardot, J.-P. et Guigon, P., 1991 - Une forteresse du $\mathrm{X}^{\mathrm{e}}$ siècle : le Camp de Péran à Plédran (Côtes-d'Armor). Revue archéologique de l'Ouest, 8, p. 123-157.

Pailler, Y., Marchand, G., Blanchet, S., Guyodo, J.-N. et Hamon, G., 2008 - Le Villeneuve-Saint-Germain dans la péninsule armoricaine : les débuts d'une enquête, in BurNEzLanotte L., Ilett, M. et Allard, P. (dir), Fin des traditions danubiennes dans le Néolithique du Bassin parisien et de la Belgique (5100-4700 av. J.-C.). Paris, Société préhistorique française (Mémoire XLIV). p. 91-111.

Pautreau, J.-P. et Maitay, C., 2007 - L'éperon barré du Camp Allaric, Aslonnes (Vienne). Trente années de recherches. XXVI congrès préhistorique de France, Avignon 2004, Paris, Société préhistorique française, vol. II, p. 359-369.

Petit, M. (dir.), 2009 - L'habitat carolingien des Sureaux à la Grande-Paroisse (Seine-et-Marne) Une communauté villageoise à l'aube de l'An Mil. Melun, Conseil général de Seine-et-Marne (Mémoires archéologiques de Seine-et-Marne, 3).

Poissonnier, B., 2002 -Pilons, broyeurs, bouchardes, marteaux et autres percuteurs : les interprétations fonctionnelles au risque de l'expérimentation, in Procopiou, H. et Treuil, R. (dir.), Moudre et broyer, l'interprétation fonctionnelle des outils de mouture et de broyage dans la préhistoire et l'antiquité, t. I : Méthodes (Actes de la table ronde internationale de Clermont-Ferrand, 1995), Paris, CTHS, p. 141-152.

Pommepuy, C., 1999 - Le matériel de mouture de la vallée de l'Aisne de l'âge du Bronze à La Tène finale : formes et matériaux. Revue archéologique de Picardie, nº 3-4, p. 115-141. 
Richard, H., Magny, M. et Mordant, C., 2007 - Environnements et cultures à l'âge du Bronze en Europe occidentale. Paris, CTHS (Documents préhistoriques, 21), 395 p.

San Juan, G., Fontugne, M., Lepaumier, H., Ghesquiere, E., Fromont, N. et Gallouin, E., 2000 - L'éperon barré Néolithique final et hallstatien de La Campagne à Basly (Calvados). Internéo, 3, p. 143-148.

San Juan, G., Ghesquiere, E., Fontugne, M., Fromont, N. et Gallouin, E., 2007 - Une enceinte fortifiée en éperon du troisième millénaire avant J.-C. à Basly dans le Calvados : résultats préliminaires, Revue archéologique du Centre de la France, $27^{\mathrm{e}}$ supplément (Agogué, O., Leroy, D. et Verjux, C. (dir.), Camps, enceintes et structures d'habitat néolithiques en France septentrionale - Actes du $24^{\mathrm{e}}$ Colloque interrégional sur le Néolithique, Orléans1999), p. 327-348.

SchweIngRuber, F. H., 1982 - Microscopic Wood Anatomy, Structural variability of Stems and Twigs in Recent and Subfossil Woods from Central Europe (2nd ed.). Teufen (Suisse), F. Flück-Wirth, 226 p.

SCHWEINGRUBer, F. H., 1990 - Anatomy of European woods : an atlas for the identification of European trees, shrubs, dwarf shrubs. Bern/Stuttgart, WSL FNP, Haupf, 800 p.

Sestier, C. et Bontemps, C., 2003 - Les bouchardes en matériaux tenaces : observations archéologiques et expérimentales. Les matières premières lithiques en préhistoire (actes de la table ronde internationale d'Aurillac, 2002). Préhistoire du Sud-Ouest (suppl. $n^{\circ}$ ), p. 307-315.

Simonin, D., 1996 - Boulancourt, Le Châtelet (Seine-et-Marne). Bilan scientifique régional d'Ile-de-France, Paris, Service régional de l'Archéologie; p. 42-43.
Thery-Parisot, I., 2001 - Économie des combustibles au Paléolithique. Paris, CNRS (Dossier de Documentation archéologique $\left.\mathrm{n}^{\circ} 20\right), 195 \mathrm{p}$.

Thomas, E. et Carn, A., 2008 - Saint-Méen-le-Grand, carte géologique de la France à 1/50 000, nº 315, Orléans, BRGM éditions, + notice $98 \mathrm{p}$.

Thomas, E., Brault, N., Carn, A., Lecerf, Y. et Rivière, J.-M., 2004 - Ploërmel, carte géologique de la France à 1/50 000, $n^{\circ} 351$, Orléans, BRGM éditions, + notice 115 p.

TineveZ, J.-Y., 1992 - Structures d'habitat du Néolithique et de l'âge du Bronze décelées récemment en Bretagne. Revue archéologique de l'Ouest, supplément $\mathrm{n}^{\circ} 5$ (actes du 17e colloque interrégional sur le Néolithique, Vannes 1990), p. 71-78.

Tinevez, J.-Y. (dir.), 2004 - Le site de La Hersonnais à Pléchâtel (Ille-et-Vilaine) : un ensemble de bâtiments collectifs du néolithique final. Paris, Société préhistorique française (coll. Travaux, 5), $172 \mathrm{p}$.

Tinevez, J.-Y., Lorho, T. et Quesnel, L., 2006 - Vestiges d'habitat du Néolithique moyen 1 à Quimper, lieu-dit KervouyecNevez (Finistère), Internéo, 6, p. 67-76.

Villard, J.-F., 2005 - La poterie, typologie et chronologie, in Le Bihan, J.-P. et Villard J.-F. (dir.), Archéologie de Quimper, matériaux pour servir l'Histoire. t. 1 De la chute de l'empire romain à la fin du Moyen Âge. Quimper, Centre de Recherche archéologique du Finistère, p. 358-389.

VISSET, L., 1989 - La tourbière de Landemarais en Parigné (Illeet-Vilaine, France). Étude pollinique, Lejeunia, 129, p. 16-26. 